\title{
Das System Erde - was bewegt die Welt?
}

Lebensraum und Zukunftsperspektiven

Herausgegeben im Auftrage der Akademie der Wissenschaften zu Göttingen von Joachim Reitner, Klaus Weber und Ute Karg

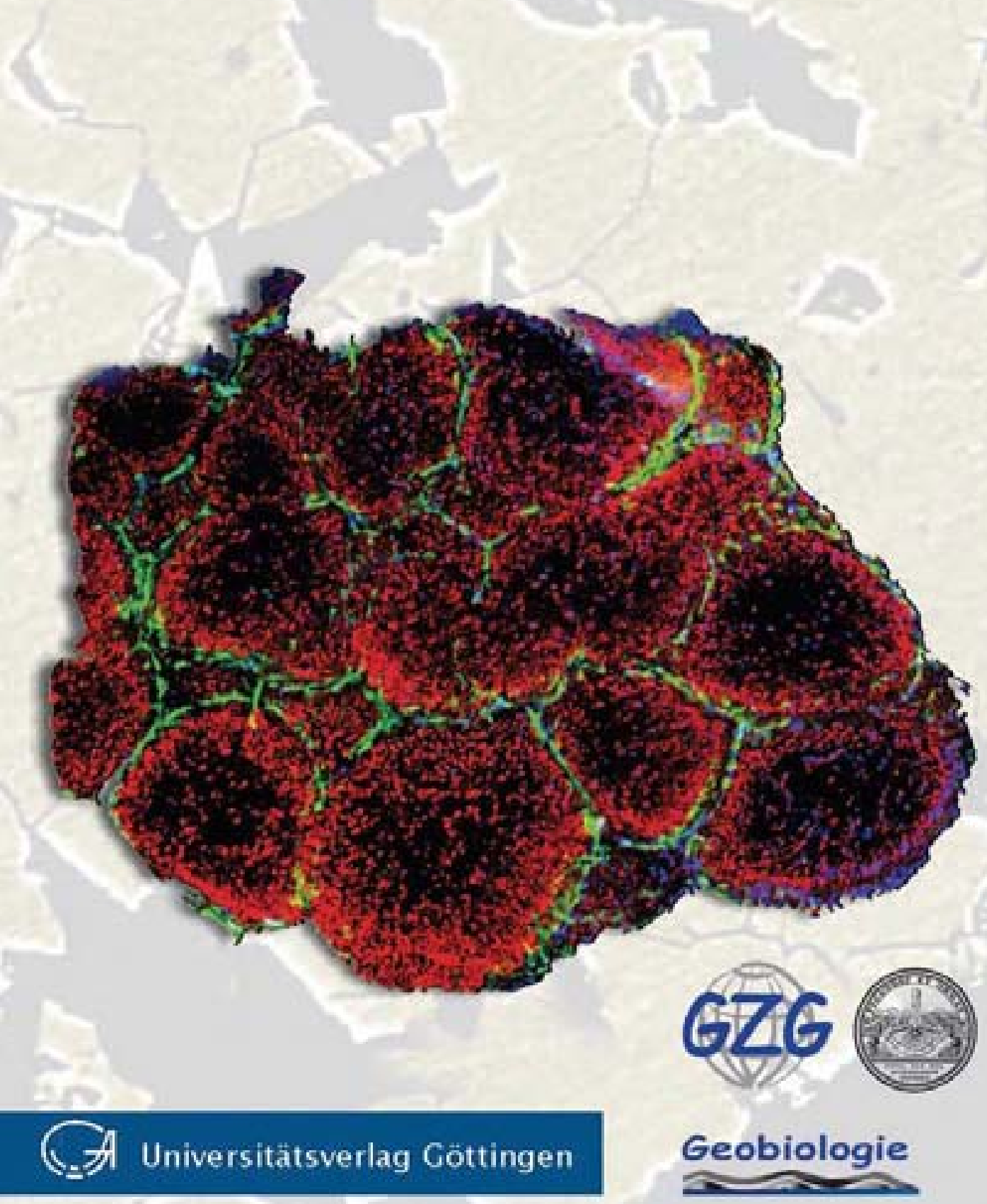



Joachim Reitner, Klaus Weber und Ute Karg (Hg.)

Das System Erde - was bewegt die Welt? 
erschienen im

Universitätsverlag Göttingen 2005 


\section{Das System der Erde - was bewegt die Welt?}

Lebensraum und

Zukunftsperspektiven

Herausgegeben im Auftrage der Akademie der Wissenschaften zu Göttingen von

Joachim Reitner, Klaus Weber und Ute Karg

Universitätsverlag Göttingen 2005 


\section{Bibliografische Information der Deutschen Bibliothek}

Die Deutsche Bibliothek verzeichnet diese Publikation in der

Deutschen Nationalbibliographie; detaillierte bibliografische Daten sind im Internet über $<$ http://dnb.ddb.de $>$ abrufbar.

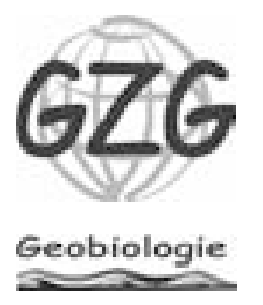

Geowissenschaftliches Zentrum der Universität Göttingen Goldschmidtstr. 3

37077 Göttingen

Tel. +49-(0)551-397951

Fax +49-(0)551-397918

uggp@gwdg.de

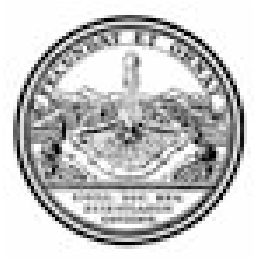

Herausgegeben im Auftrage der Akademie der Wissenschaften zu Göttingen

Theaterstraße 7

37073 Göttingen

Tel. $+49(0) 551 / 39-5362$

Fax +49 (0)551/39-5365

udeppe@gwdg.de

Satz und Layout Ute Karg

Titelgestaltung Conny Kaubisch und Karsten Riedl

Umschlaghintergrund: Polierter Dünnschliff mit Flussspat-Kristallen

Titelabbildung: Epifluoreszenz-Bild von Kolonien anaerob Methanoxidierender

Mikroorganismen aus dem Schwarzen Meer. Diese Mikroorganismen sind aktiv an der Bildung von Karbonatgesteinen beteiligt. Die Mikroorganismen wurden über die sog. Fluoreszenz in situ Hybridisierung (FISH) bestimmt, bei der bestimmte Abschnitte des 16S RNS Gens angefärbt werden. Die grüne Farbe detektiert hier Archaea, die Methan anaerob oxidieren (ANME2) und die rote Farbe markiert Sulfatreduzierende Bakterien. Der DNS Farbstoff DAPI ist blau und wird zur Kontrolle der FISH-Daten eingesetzt.

(C) Alle Rechte vorbehalten, Universitätsverlag Göttingen 2005

ISBN 3-938616-07-5 


\section{Inhaltsverzeichnis}

Vorwort

Die Anfänge des Lebens auf der Erde: Empirische Befunde der frühen geologischen Überlieferung

Manfred Schidlowski

Die sedimentäre Haut unseres Planeten

Reinhard Gaupp 37

Wenn Kristalle fließen und Schmelzen hüpfen

Sharon L. Webb 57

Isotope, die Gene der Gesteine

Bent Tauber Hansen .73

Fernerkundung der Erde - Neue Sensoren und innovative Techniken

Martin Kappas

Die überwundene Schwerkraft - eine halbe Milliarde Jahre WirbeltierEvolution

Annette Broschinski

Die Sintflut aus geologischer Sicht

Klaus Weber

Geologie und Paläontologie im Rahmen der kulturellen Entwicklung

Otto H. Walliser.

Anschriften der Autoren 



\section{Vorwort}

Der Planet Erde ist ein nur in Ansätzen verstandenes, komplexes und vernetztes System von geologischen, biologischen und kosmischen Prozessen und somit ein interessantes und vielseitiges Forschungsobjekt.

Die hier vorgestellten Beiträge waren Teil einer öffentlichen Ringvorlesung der Akademie der Wissenschaften zu Göttingen und der Georg-August-Universität Göttingen im WS 2002/2003, organisiert von den Herausgebern dieses Buches. Ziel dieser Ringvorlesung war es, einige Facetten der vielfältigen geowissenschaftlichen Forschung, philosophische Aspekte und Elemente einer geowissenschaftlich orientierten Forschungspolitik einer breiten Öffentlichkeit vorzustellen. Insgesamt hatten sich 16 Kolleginnen und Kollegen an der Ringvorlesung beteiligt. Die Durchführung und Organisation der Ringvorlesung wurde dankenswerterweise vom Universitätsbund, der Universitätsstiftung und von der Akademie der Wissenschaften zu Göttingen finanziell unterstützt.

Die Autoren der Beiträge sind weltweit anerkannte Spezialisten und haben in ihrer Forschungsarbeit geowissenschaftliche Meilensteine gesetzt.

Prof. Dr. Manfred Schidlowski (Max-Planck-Institut für Chemie, Mainz) hat sich mit der Biogeochemie der frühen Erde beschäftigt und grundlegende Arbeiten zur Verteilung von stabilen Kohlenstoffisotopen in den ältesten Sedimentgesteinen der Erde durchgeführt.

Prof. Dr. Reinhard Gaupp (Allgemeine und Historische Geologie, Universität Jena) ist Sedimentologe und beschäftigt sich mit der „,sedimentären Haut unseres Planeten“. Die Sedimente unseres Planeten sind mit die wichtigste Rohstoffquelle und somit auch essentiell für die zukünftige Entwicklung der Menschheit auf der Erde.

Prof. Dr. Sharon Webb (Experimentelle und Angewandte Mineralogie, Geowissenschaftliches Zentrum der Universität Göttingen) ist eine anerkannte Spezialistin für Gläser und vulkanische Schmelzen. Ihre Arbeiten erlauben Einblicke in die Welt der Schmelzen und Kristalle und somit auch Einblicke in das tiefe Innere unseres Planeten.

Prof. Dr. Bent Hansen (Isotopen-Geochemie, Geowissenschaftliches Zentrum der Universität Göttingen) ist Isotopen-Geochemiker und beschäftigt sich mit schweren radiogenen und stabilen Isotopen, die wichtig sind für das grundlegende Verständnis geologischer Prozesse und deren zeitlichen Rahmen. Ohne die Arbeit mit Isotopen könnten kosmische und geologisch wichtige Stoffkreisläufe nicht rekonstruiert werden.

Prof. Dr. Martin Kappas (Geographisches Institut der Universität Göttingen, Kartographie, GIS und Fernerkundung) ist Geoinformatiker und beschäftigt sich mit den vielfältigen Facetten der Fernerkundung. Ein Schwerpunkt seiner Forschung ist die Satellitenfernerkundung im Verbund mit Entwicklungen einer 
modernen Informations- und Kommunikationstechnologie zum Verständnis des komplexen „Systems Erde“.

Dr. Annette Broschinski ist Oberkustodin am Niedersächsischen Landesmuseum für Naturkunde Hannover und beschäftigt sich mit der Paläobiologie von Wirbeltieren. Ihr Spezialgebiet ist die Biomechanik und die Evolution der Bewegungsabläufe bei Wirbeltieren.

Prof. Dr. Klaus Weber (Strukturgeologie, Geowissenschaftliches Zentrum der Universität Göttingen) ist Strukturgeologe und beschäftigt sich mit gesteinsphysikalischen Phänomenen, aber auch mit Mythen, die einen geowissenschaftlichen Hintergrund besitzen. Der Sintflut-Mythos hat möglicherweise einen geowissenschaftlichen Hintergrund. Von ihm wird dieses Thema kritisch beleuchtet.

Prof. Dr. Otto Heinrich Walliser (em. Professor für Paläontologie, Geowissenschaftliches Zentrum der Universität Göttingen) beschäftigt sich neben grundlegenden Arbeiten zur Paläontologie mit Geschichte der geologischen Wissenschaften, insbesondere mit deren Anfängen und philosophischen Grundlagen.

Den Herausgebern dieses Buches ist es ein Anliegen, einige wichtige und interessante Aspekte der Geowissenschaften einer breiteren Öffentlichkeit zu vermitteln. Es besteht nicht der Anspruch auf Vollständigkeit dieses Themenkomplexes. Die Herausgeber wollen jedoch Anstöße geben, sich intensiver mit dem Themenfeld Geowissenschaften zu beschäftigen. Die ausgewählten Beiträge sollen dem Leser helfen, sich in der Vielfalt der Facetten dieses Forschungsbereiches zurechtzufinden.

Joachim Reitner \& Klaus Weber, April 2005 


\title{
Die Anfänge des Lebens auf der Erde: Empirische Befunde der frühen geologischen Überlieferung
}

\author{
Manfred Schidlowski
}

\section{Einführung}

Die Frage nach der Entstehung des Lebens ist - soweit unsere geschichtliche Überlieferung reicht - in allen menschlichen Kulturkreisen und Denksystemen gestellt worden. Es handelt sich somit um eine typisch transkulturelle Fragestellung, die offenbar zum Nachdenken des Menschen über sich selbst gehört. Bis in die jüngste Vergangenheit ist diese Frage fast ausschließlich spekulativ angegangen worden, meist im Rahmen oder als Teil religiöser Kosmogonien und verwandter Denkansätze (vgl. Eliade, 1964), wobei diese Vorstellungen im abendländischen Kulturkreis vom biblischen Schöpfungsbericht dominiert waren.

Im Gegensatz dazu ist der naturwissenschaftlich-empirische Arbeitsansatz relativ jung. Nach ersten tastenden Annäherungen an das Problem etwa durch William Harvey (1578-1657), den Entdecker des Blutkreislaufes (,omne vivum ex ovo') rückte dieser Fragenkreis erst mit der Harnstoffsynthese durch Friedrich Wöhler (1800-1882) im Jahre 1828 wieder in den Vordergrund. Louis Pasteur (1822-1895) konnte bald darauf den Nachweis erbringen, dass in unserer heutigen Welt keine spontane oder „Urzeugung“ möglich ist, dass also heute letztlich Leben nur aus Leben entstehen kann (,omne vivum e vivo). Höhepunkte in der weiteren Verfolgung dieser Fragen waren die Koazervat-Hypothese von Oparin (1924) und 
die abiotische Synthese von Aminosäuren (Miller, 1953) sowie der Nachweis von zellular strukturierten Mikrofossilien in präkambrischen Sedimentgesteinen (Barghoorn \& Tyler, 1965). Eine wissenschaftsgeschichtlich skurrile Facette dieser Forschungsrichtung war ihre massive Förderung in der ehemaligen Sowjetunion zur Unterstützung atheistischer Volkserziehungsprogramme.

Wenn wir mit unserem gegenwärtigen Wissensstand die Frage der Lebensentstehung über den naturwissenschaftlich-empirischen Arbeitsansatz gezielt angehen, dann können wir das Problem von zwei Seiten einengen. Zum ersten wissen wir heute, dass schon das astrophysikalische Milieu interstellarer Staub- und Molekülwolken durch eine äußert reaktive Kohlenstoffchemie gekennzeichnet ist, die photochemisch (vor allem durch solares Ultraviolett) angetrieben wird (Greenberg, 1984). Zu den im Weltraum molekülspektroskopisch nachgewiesenen Verbindungen gehören viele Substanzen, die wichtige Vorstufen bei der präbiotischen Synthese von Zuckern, Proteinen und Nukleinsäuren bilden, wie etwa Formaldehyd $\left(\mathrm{CH}_{2} \mathrm{O}\right)$, Blausäure $(\mathrm{HCN})$, Acetaldehyd $\left(\mathrm{CH}_{3} \mathrm{CHO}\right)$, Cyanoacetylen $\left(\mathrm{HC}_{2} \mathrm{CN}\right)$ u. a. (Irvine \& Knacke, 1989). Allgemein lässt sich sagen, dass der Stoff, aus dem das Leben gemacht ist, bereits im interstellaren Medium existierte, das ja selbst wiederum seine Entstehung einer Supernova, d. h. der Explosion eines Fixsterns, verdankt. Bei der späteren Kondensation solcher Staubwolken geht dann das organische Material in den chondritischen Stoffbestand neugebildeter Sonnensysteme und speziell ihrer Planeten ein und steht damit einer möglichen Evolution von Lebensprozessen zur Verfügung - eine Erkenntnis, die Sir James Jeans (1931) mit dem Stoßseufzer quittiert hat, dass unsere Körper aus der Asche längst erloschener Sterne erschaffen sind (,our bodies are formed from the ashes of long dead stars).

Zusammenfassend lässt sich somit die begründete Ansicht vertreten, dass Leben - oder anders formuliert, die Chemie der Eiweißkörper und Nukleinsäuren - in einem bestimmten Stadium der kosmischen oder planetarischen Evolution als eine qualitativ neue Existenzform der Materie entstanden ist, die sich auf eine Reihe besonderer Eigenschaften stützt. Neben einem eng begrenzten Stoffbestand (mit massiver Kohlenstoff-Präferenz) gehört dazu vor allem der Wechsel von einer stochastischen Chemie (wo Substanzen nach Zufall und Wahrscheinlichkeit miteinander reagieren) zu einer Art „algorithmischer“ Chemie, bei der die Reaktionsabläufe vorgegebenen Mustern folgen, wobei Homochiralität und Selbstreplikation zu den entscheidenden Neuerungen zählten. Nach dieser Vorstellung wäre das Auftreten von Leben eine zwangsläufige Konsequenz des kombinatorischen Potentials der Materie im allgemeinsten Sinne und damit ein kosmischer Imperativ.

Eine zweite Annäherung an das Problem der Lebensentstehung wäre der Versuch, die Spuren des Lebens in der geologischen Überlieferung eines Einzelplaneten wie der Erde systematisch zurückzuverfolgen und dabei auf mögliche Anfänge zu stoßen. Diese Vorgehensweise bildet das eigentliche Thema des folgenden Diskurses und stützt sich auf eine Fülle empirischer Befunde, wie sie insbesondere in den letzten Jahrzehnten zusammengetragen worden sind. 


\section{Die Anfänge des Lebens auf der Erde}

Im Falle unserer Erde gehen die Informationen zur Frühgeschichte des Lebens nur bis zur Zeitmarke von 3,8 Mrd. Jahren zurück, weil hier der Informationsträger, die sedimentäre Überlieferung, ausfällt. Bekanntlich ist in der Erdgeschichte Zeit immer durch Stoff belegt, wobei die Abfolge geologischer Formationen letztlich materialisierte Zeit repräsentiert. Die in den einzelnen Zeitintervallen abgelagerten Sedimentgesteine dienen dabei als Trägermatrix für die verschiedensten Kategorien von Informationen, von denen die biologisch relevanten in Abb. 1 zusammengefasst sind. Wie hier ersichtlich, liefert uns die geologische Überlieferung mehrere Arten von paläontologischer und biochemischer Evidenz, die Rückschlüsse auf die frühe Entwicklung des Lebens auf unserem Planeten erlauben und denen wir in der Folge detailliert nachgehen wollen. Die ältesten Informationsträger sind dabei die Sedimente der Isua-Serie (3,6-3,8 Mrd. Jahre) von West-Grönland, die allerdings eine leichte metamorphe Überprägung erfahren haben, bei der fossile Lebensspuren weitgehend verwischt werden können. Die ältesten unveränderten Sedimente gehen bis zu 3,5 Mrd. Jahren zurück und treten reliktisch in fast allen archaischen Kratonen auf, besonders im nordwestlichen Australien (Pilbara-Supergruppe) und in Südafrika (Swaziland-Supergruppe).

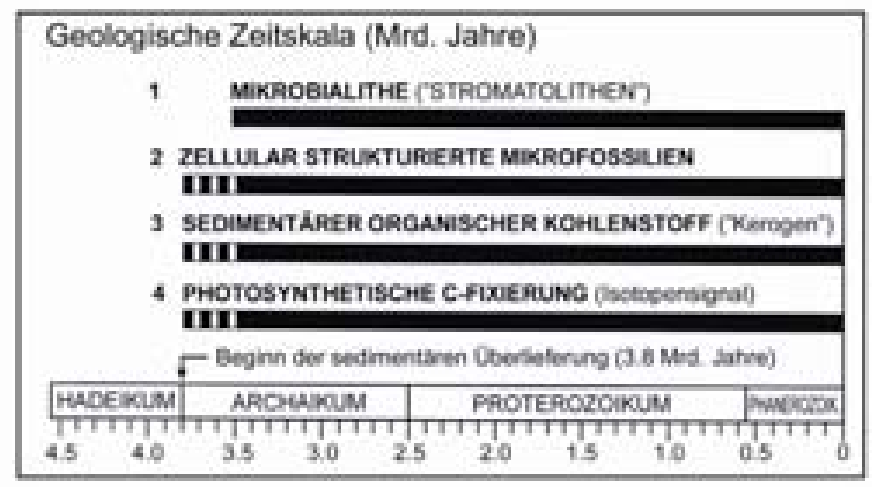

Abb. 1: Die wichtigsten Kategorien paläobiologischer Eviden₹ über 3,8 $\times 10^{9}$ (Milliarden) Jahre Erdgeschichte. Das „hadeische“ Äon am Beginn der Zeitskala umfasst die Zeit von der Bildung des Planeten (4,56 Mrd. Jahre) bis zum Einsetzen der sedimentären Überlieferung; Archaikum und Proterozoikum werden auch konventionell als „Präkambrium“ zusammengefasst. Der älteste Teil der archaischen Überlieferung ( $t>3,5 \mathrm{Mrd}$. Jahre) ist von der Gesteinsmetamorphose überprägt worden (gebrochene Linien). Das hat den Erhaltungszustand von Mikrofossilen (2) dramatisch verschlechtert, zu einer weitgehenden Graphitisierung der organischen Koblenstoffkomponente geführt (3) und dabei auch die ${ }^{13} \mathrm{C} /{ }^{12} \mathrm{C}$-Signatur des organischen Kohlenstoffs verschoben, die im unveränderten Gestein die Isotopenfraktionierung bei der Photosynthese anzeigt (4). Fossile Relikte von feinschichtigen mikrobiellen Ökosystemen (,Stromatolithen") sind bisher nur bis zur Zeitgrenze von 23,5 Mrd. Jahren nachgewiesen worden (1). 
Zu den wichtigsten Dokumenten frühen irdischen Lebens zählen zwei Kategorien paläontologischer Evidenz, nämlich (1) Mikrobialithe (sog. „Stromatolithen“) und (2) zellular strukturierte Mikrofossilien (Abb. 1). Bei den ersteren handelt es sich um biosedimentäre Strukturen, die auf das mattenbildende Verhalten benthonischer Mikroorganismen zurückgehen (Abb. 2), wobei es sich überwiegend um photoautotrophe Prokaryonten wie Cyanobakterien („Blaualgen“) handelt. Solche „Prokaryonten“ (mit Zellen ohne morphologisch strukturierten Zellkern) bauen ihre Körpersubstanz direkt aus Kohlendioxid $\left(\mathrm{CO}_{2}\right)$ und Wasser $\left(\mathrm{H}_{2} \mathrm{O}\right)$ mit Hilfe eingefangener Lichtquanten (hv) auf, wobei molekularer Sauerstoff $\left(\mathrm{O}_{2}\right)$ als Stoffwechselprodukt freigesetzt wird, d. h.

$$
2 \mathrm{H}_{2} \mathrm{O}+\mathrm{CO}_{2} \stackrel{h v}{\longrightarrow} \mathrm{CH}_{2} \mathrm{O}+\mathrm{H}_{2} \mathrm{O}+\mathrm{O}_{2} \quad \Delta \mathrm{G}_{0}^{\prime}=+470,7 \mathrm{~kJ} \quad \text { (Gl. 1) }
$$

Die Verbindung $\mathrm{CH}_{2} \mathrm{O}$ steht dabei für die neugebildete organische Substanz (Kohlenhydrat). Die geschichteten Strukturen derartiger „Stromatolithen“ konservieren die Aufeinanderfolge feinlaminierter Bakterien- und Algenrasen, die gewöhnlich die Grenzfläche zwischen Sediment und Wasser besiedeln und wobei die jeweils jüngere Generation die ältere überlagert (Abb. 2 und 3). Die Konservierung der charakteristischen Laminarstruktur derartiger Mikrobialithe beruht letztlich auf der Wechselwirkung der biologisch aktiven Mikrobenmatte mit ihrer sedimentären Umwelt. Die Fossilisation der einzelnen Laminae erfolgt dabei vor allem durch Agglutination bzw. Bindung oder biologisch induzierte Ausfällung ausgewählter Mineral- und Gesteinskomponenten.

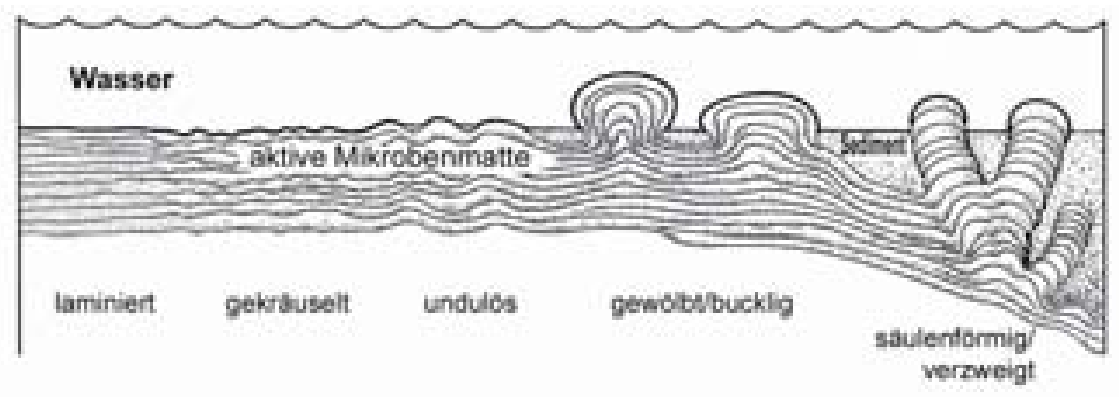

Abb. 2: Überblick über das morphologische Formeninventar von laminierten mikrobiellen Ökosystemen, die die Grensfläche von Sediment und Wasser in geeigneten wässerigen Habitaten besiedeln. Diese Abfolgen von Mikrobenmatten werden als ,Stromatolithen "fossil überliefert und dokumentieren die ehemalige Anwesenheit von bodenbewohnenden Mikroorganismen (meist Cyanobakterien). 


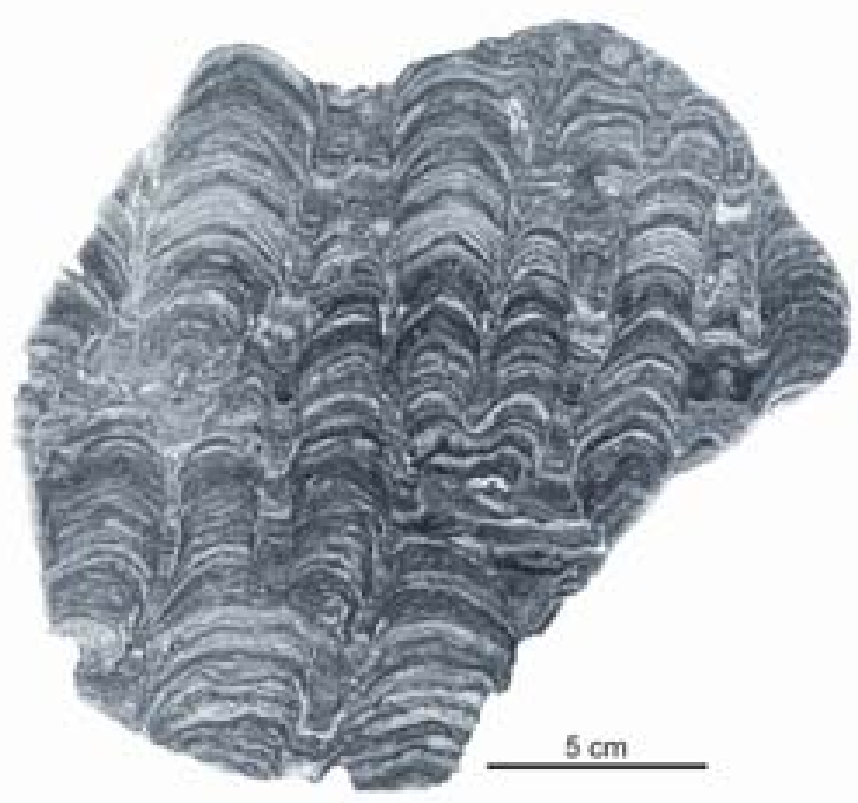

Abb. 3: Typischer Stromatolith aus dem frühen Proterozoikum des Labrador-Beckens (Canada). Das abgebildete Stück zeigt eine digitale Verzweigung an der Basis, die nach oben in ein System subparalleler Säulen übergeht. Man beachte die auffällige Laminierung innerhalb der Gesamtstruktur, die die Generationenfolge nachwachsender Mikrobenmatten konserviert hat.

In der erdgeschichtlichen Überlieferung bilden Mikrobialithe die auffälligste Manifestation fossilen Mikrobenlebens, wobei die ältesten Vorkommen bis annähernd 3,5 Mrd. Jahre zurückgehen. Das bedeutet, dass bodenbewohnende (,,benthonische") Prokaryonten bereits in geeigneten wässerigen Biotopen des Archaikums weit verbreitet waren. Sowohl das morphologische Formeninventar der ältesten Stromatolithenstrukturen als auch die Mikrofossilführung der umgebenden (und gleichaltriger) Sedimente erlauben eine weitgehende Rekonstruktion der frühen mikrobiellen Ökosysteme und zeigen, dass die archaischen Produzenten von biosedimentären Strukturen stromatolithischen Typs sich offenbar kaum von ihren jüngeren Nachfahren (einschließlich der heutigen) unterschieden haben. Es scheint heute weitgehend sicher, dass die ältesten mattenbildenden Mikroorganismen filamentartige einzellige Prokaryonten waren, die sowohl die Fähigkeit zur photosynthetischen Kohlenstoff-Fixierung als auch zu phototaktischen Reaktionen besaßen (Walter, 1983). Die kontinuierliche Überlieferung vom Archaikum bis zur Gegenwart bezeugt weiterhin ein erstaunliches Maß von Gleichförmigkeit und Konservatismus in der Physiologie und kommunalen Organisation (wie Matten- und sonstigen Koloniebildungen) von prokaryotischem Mikrobenthos über 3,5 Mrd. Jahre Erdgeschichte. Trotz gelegentlicher Konvergenzen von biologisch induzierten mit anorganisch entstandenen Feinlaminierungen (etwa evapori- 
tischer oder mikroklastischer Herkunft) im Sediment, die manchmal eine Differentialdiagnose erfordern (Grotzinger \& Knoll, 1999), gilt es nach unserem heutigen Kenntnisstand als hinreichend gesichert, dass speziell die ältesten Stromatolithen-Vorkommen zu den wichtigsten Dokumenten der Frühgeschichte des Lebens auf unserem Planeten gehören (Hofmann, 2000).

Neben diesen bereits mit bloßem Auge erkennbaren Relikten vergangenen Mikrobenlebens gibt es noch eine zweite (mikroskopische) Kategorie früher paläontologischer Evidenz in Form von zellular erhaltenen Mikrofossilen. Der Nachweis fossiler Mikroorganismen geht mit Sicherheit über mehr als 3 Mrd. Jahre Erdgeschichte zurück, wobei sich die Anfänge - je nach Auffassung der betreffenden Denkschulen - entweder bei 3,5 oder 3,8 Mrd. Jahren im Dunkel der sedimentären Überlieferung verlieren (Abb. 1).

Während das Auftreten zellular erhaltener Mikrobenfaunen im proterozoischen Zeitabschnitt der präkambrischen Erdgeschichte durch eine Fülle von Vorkommen belegt ist (Rozanov, 2002), bereitet die eindeutige Identifizierung bakterieller Mikrofossilen in geologisch älteren Gesteinen zunehmende Schwierigkeiten. Speziell in den Sedimenten des frühen Archaikums wurden durch fortschreitende diagenetische und metamorphe Umwandlungsprozesse in der Mineralmatrix des umgebenden Gesteins die Primärmorphologien solcher feinen organischen Mikrostrukturen immer stärker verwischt, was zu einem weitgehenden Verlust von Konturen und anderen kritischen morphologischen Details führte. Die Extreme derartiger „Verstümmelungen“ primärer Zellstrukturen manifestieren sich in der Folge in Form sog. „Dubiofossilien“ von wechselnder (und manchmal zweifelhafter) Aussagekraft. Für die Beurteilung der biologischen Herkunft solcher zellartiger Strukturen in archaischen Sedimenten ist eine abgestufte Liste von Selektionskriterien vorgeschlagen worden (Buick, 1991). Danach sollte für authentische Mikrofossilien sichergestellt sein, dass sie (1) primärer Bestandteil des Trägergesteins sind (bezeugt durch ihre Präsenz im petrographischen Dünnschliff), (2) in großer Individuenzahl auftreten, (3) Relikte von organischem Kohlenstoff führen sowie (4) die Größendimension biologischer Zellen nicht unterschreiten und auBer einem zentralen Hohlraum möglichst noch differenzierte strukturelle Details zeigen, die sich nur schwer als Resultat anorganischer Prozesse deuten lassen. Aus prinzipiellen Erwägungen sollte außerdem jede Form von vermeintlicher Evidenz aus metamorphen Sedimenten unberücksichtigt bleiben.

Trotz auffälliger Defizite in der paläontologischen Überlieferung besonders des frühen Archaikums sind jedoch aus verschiedenen archaischen Terrains Mikrobenfloren beschrieben worden, die den obigen Kriterien annähernd genügen. Neben Vorkommen aus dem südlichen Afrika (Pflug, 1967; Muir \& Grant, 1976; Knoll \& Barghoorn, 1977; Walsh, 1992; Westall et al., 2001) sind hier vor allem die in Kieselsteinerhaltung vorliegenden Mikrofloren aus der WarrawoonaGruppe des Pilbara-Kratons von Westaustralien zu nennen (Abb. 4), die mit einem Alter von annähernd 3,5 Mrd. Jahren als die am besten erhaltenen Mikrobengesellschaften der frühen geologischen Überlieferung interpretiert worden sind (Schopf \& Packer, 1987; Schopf, 1993). Auffallend in diesen Mikrofloren sind so- 
wohl die fadenartigen (trichomischen) als auch die rundlichen (kokkoiden) Morphotypen, die bereits das Formeninventar cyanobakterieller Vorgängerfloren im Proterozoikum dominiert hatten. Die septenartig unterteilten Filamente (Abb. 4) lassen sich dabei als fossile Trichome erklären, die auf fadenförmige Cyanobakterien oder auch primitivere Prokaryonten (wie etwa Flexibakterien) zurückgehen, während die kokkoiden Aggregate nach Meinung von Schopf \& Packer (1987) nur eine Deutung als fossile Cyanobakterien erlauben.

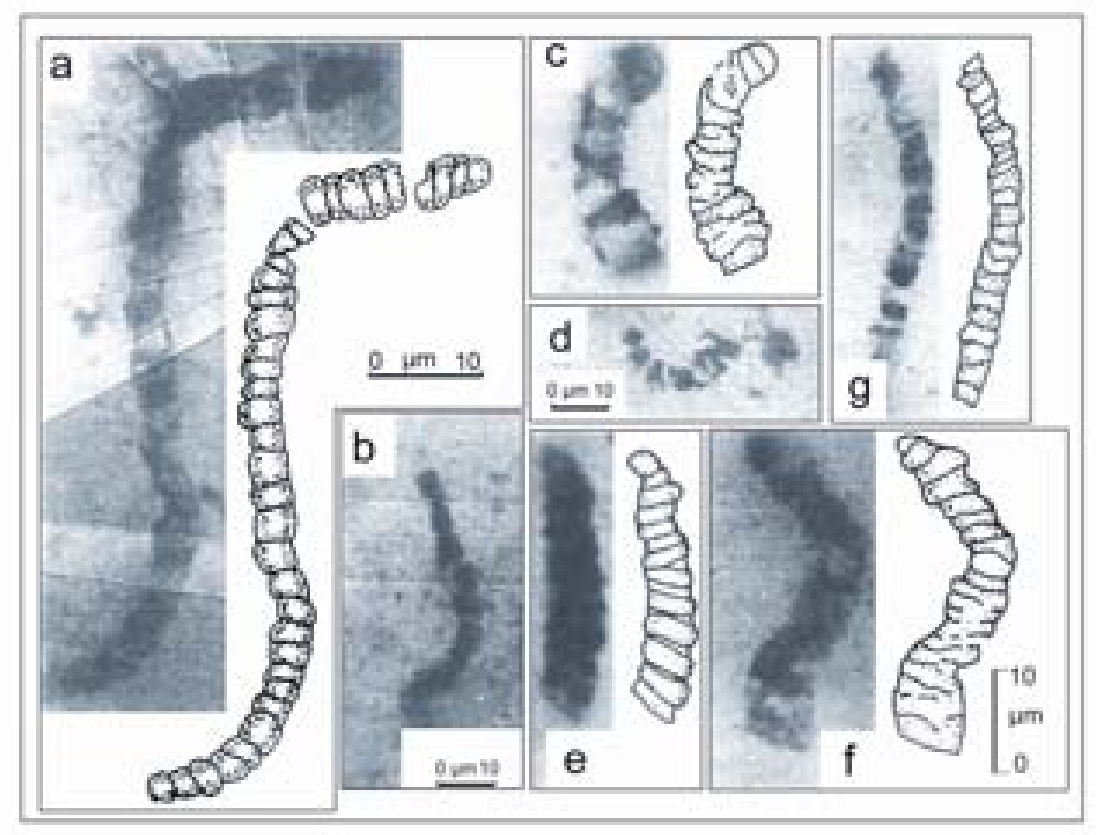

Abb. 4: Filamente mit septenartiger Unterteilung, die morphologische Beziehungen zu Cyanobakterien nahelegen. Diese ₹. Zt. umstrittenen „biomorphen "Mikrostrukturen aus dem 3,46 Mrd. Jahre alten "Apex Chert" im unteren Teil der Warrawoona-Gruppe des Pilbara-Kratons (WestAustralien) hat Schopf (1993) als Primaevifilum amoenum (A, B), Archaeoscillatoriopsis disciformis $(C, D, E, F)$ und Primaevifilum delicatulum $(G)$ beschrieben.

Angesichts der bemerkenswerten Differenzierung der inzwischen bekannt gewordenen archaischen Mikrofloren müssen wir zwangsläufig annehmen, dass sich die Abstammungslinien der beobachteten mikrobiellen Morphotypen bereits wesentlich vor der Zeitmarke von 3,5 Mrd. Jahren herausgebildet hatten. Wir können deshalb mit guten Gründen postulieren, dass Vorläuferfloren bereits in dem Zeitabschnitt existierten, in dem die geologische Überlieferung lückenhaft und durch Gesteinsmetamorphose überprägt ist. In diesem Zusammenhang haben Funde von kohlenstoffhaltigen Zellstrukturen in den 3,8 Mrd. Jahre alten Metasedimenten des Isua-Grünsteingürtels von W-Grönland beträchtliche Aufmerksam- 
keit erregt. Die biologische Natur des in dieser Serie bevorzugt auftretenden Morphotyps, den Pflug (1978) als Isuasphaera isua (Abb. 5) beschrieben hat, ist in der Folge massiv bestritten worden (Bridgwater et al., 1981). Das Hauptargument der Kritiker war dabei die Unwahrscheinlichkeit der Erhaltung feinster Zellstrukturen in einem Muttergestein, das einer Metamorphose vom Grade der Amphibolit-Fazies ausgesetzt war.

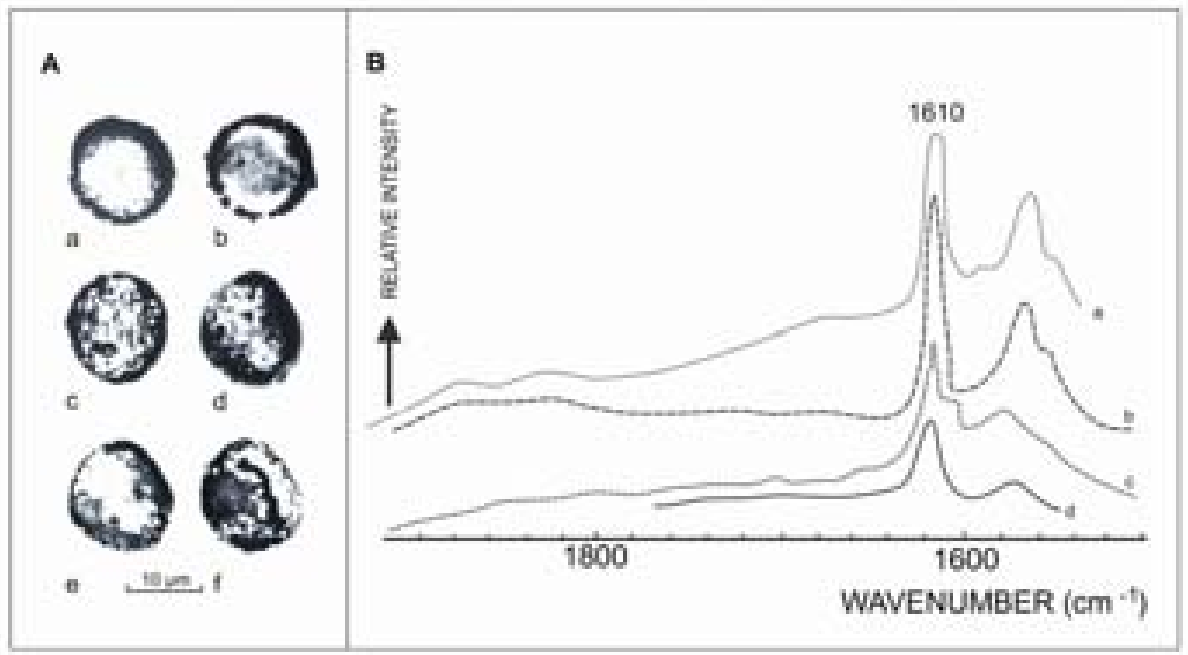

Abb. 5: A. Vergleich von Huroniospora sp. aus der 2 Mrd. Jabre alten Gunflint-Eisenformation von Ontario (a-c) mit Isuasphaera sp. aus den 3,6-3,8 Mrd. Jabre alten Metasedimenten des Isua-Grünsteingürtels von West-Grönland (d-f). Bei dem auffälligen Randsaum einiger dieser biomorphen Strukturen könnte es sich um Reste der ursprpünglichen Zellwand handeln. B. LaserRaman-Spek.tren von Huroniospora sp. als isoliertes Objekt (a) und in Dünnschliffeinbettung (b) verglichen mit entsprechenden Spektren von Isusaphaera sp. (c, d). Die Spektren bezeugen eine weitgehende Übereinstimmung in der Zusammensetzung der jeweiligen organischen Restsubstanzen und unterscheiden sich nur in ihrer Intensität. Das auffällige Maximum bei $1610 \mathrm{~cm}^{-1}$ zeigt eine aromatische Doppelbindung von C-Atomen innerhalb der vorliegenden Molekülstruktur an. Nach Pflug (1987).

Es gibt jedoch eindeutige Hinweise dafür, dass sowohl Makro- wie Mikrofossilien mittleren Graden von Gesteinsmetamorphose in qualitativ abgestuften Erhaltungszuständen durchaus widerstehen können (z. B. Ivanova et al., 1988). Deshalb scheint es nicht zulässig, eine biologische Affinität zellartig strukturierter Elemente aus den Isua-Sedimenten a priori auszuschließen, zumal die Mikromorphologien des Isuasphaera-Typs eine auffällige Übereinstimmung mit Strukturen zeigen, die aus proterozoischen Gesteinen als Huroniospora sp. beschrieben worden sind und deren biologische Herkunft allgemein anerkannt ist (siehe Abb. 5). Trotz bestehender Unsicherheiten in der Deutung der aus den Isua-Sedimenten beschriebenen „biomorphen“ Mikrostrukuren und möglichen Konvergenzen mit rein 
mineralogischen Phänomenen lässt sich nicht ausschließen, dass das Formeninventar als Ganzes Elemente einer strukturell degenerierten Mikrobengesellschaft enthält, wie man sie als Resultat einer metamorphen Beschädigung einer bakteriellen Mikroflora vom Warrawoona-Typ erwarten könnte. In jedem Falle wäre die Existenz mikrobieller Ökosysteme zu Isua-Zeiten nicht nur kompatibel mit dem Kohlenstoffgehalt und dem ${ }^{13} \mathrm{C} /{ }^{12} \mathrm{C}-$ Verhältnis der Isua-Serie, sondern geradezu eine Voraussetzung dafür.

Während die 3,8 Mrd. Jahre alten Isua-Morphotypen bereits seit langem kontrovers diskutiert wurden (Bridgwater et al., 1981; Schopf \& Walter, 1983; u. a.), hat sich die jüngste Kritik auch gegen die in verkieselter Form erhaltene Mikrobengemeinschaft der Warrawoona-Gruppe gerichtet, die mit einem Alter von fast 3,5 Mrd. Jahren als Musterbeispiel einer archaischen Mikroflora galt (Schopf, 1999). Brasier et al. (2002) haben nicht nur die Eignung des hydrothermalen Bildungsmilieus des hier fossilführenden „Apex Chert“ als primäres Mikrobenhabitat infrage gestellt, sondern darüber hinaus die Mikromorphologie der septenartig unterteilten Filamentstrukturen als mineralogische Artefakte gedeutet und damit die Warrawoona-Mikroflora als Ganzes - einschließlich der postulierten Cyanobakterien-Beziehung - in Zweifel gezogen. Außerdem bestritten die Autoren den diagnostischen Wert Laser-Raman-spektroskopischer Untersuchungsmethoden, die Schopf et al. (2002) benutzt hatten, um über die Kongruenz von Kohlenstoffverteilung und Fossilmorphologie die biologische Herkunft dieser Strukturen weiter abzusichern.

Diese z. Zt. laufenden Kontroversen über die möglicherweise ältesten fossilen Lebenszeugnisse sind ein Indiz dafür, dass sich die entsprechenden Untersuchungen an den Grenzen unseres Wissens bewegen, die im Augenblick offenbar noch Raum für entgegengesetzte Standpunkte lassen. Ein Beobachter der Szene kann sich jedoch des Eindrucks kaum erwehren, dass neben einer (sicherlich zulässigen) unterschiedlichen Wichtung einzelner Kategorien von Evidenz die Meinungswillkür des jeweiligen Bearbeiters oder der betreffenden Schule eine entscheidende Rolle bei der Ausdeutung der Befunde spielt. Obwohl unbestreitbar ist, dass weitere petrographische, mikrostrukturelle und mikroanalytische Untersuchungen notwendig sind, um die kognitiven Grundlagen für die Identifizierung fossiler Mikroorganismen genauer abzuklären (insbesondere in metamorph überprägten Gesteinen), spricht das Gesamtbild der gegenwärtig vorliegenden paläontologischen und biogeochemischen Evidenz doch ziemlich eindeutig für eine frühe Existenz von Leben auf der Erde. Selbst eine Diskreditierung der Warrawoona-Mikroflora würde das Bild nicht wesentlich ändern, da die Validität der annähernd gleichaltrigen (3,3-3,5 Mrd. Jahre) südafrikanischen Befunde davon nicht betroffen wäre. Die gegenwärtige Kontroverse um diese Mikroflora dürfte somit keine grundlegende Revision unserer Zeitvorstellungen zur frühen biologischen Evolution erfordern, wie sie in den letzten Jahren entwickelt worden sind (Schidlowski, 1993, 1998; Schopf 1999; Nisbet \& Sleep, 2001), abgesehen von Unsicherheiten über den Zeitpunkt des ersten Auftretens von Cyanobakterien. Im Falle der noch älteren grönländischen (Isua-)Evidenz mag sicherlich der Einwand 
erlaubt sein, ob der überlieferte Erhaltungszustand der biomorphen Mikrostrukturen eine formelle taxonomische Beschreibung rechtfertigt, aber eine pauschale Ablehnung dieser Befunde unter Verkennung ihres möglichen diagnostischen Potentials wäre klare Wissensverweigerung. Da jede Weiterentwicklung des optischen und mikroanalytischen Instrumentariums die Grenzen unserer Erkenntnismöglichkeiten verschiebt, wäre es naiv, unseren heutigen Kenntnisstand als letzte Wahrheit anzusehen.

\section{Biogeochemische Zeugnisse frühen irdischen Lebens}

Abgesehen von morphologischen oder „strukturierten“ Resten hinterlassen Lebewesen auch chemische Spuren ihrer einstigen Existenz. Ihre Körpersubstanz wird nach dem Tode überwiegend bakteriell abgebaut, wobei der Kohlenstoffanteil fast völlig zu Kohlendioxid $\left(\mathrm{CO}_{2}\right)$ remineralisiert wird. In der Regel entgehen nur wenige Promille bis maximal ein Prozent der ursprünglichen Kohlenstoff-Fraktion diesem Remineralisierungsprozess, die dann in der Folge als sedimentärer organischer Kohlenstoff in der irdischen Gesteinshülle gespeichert werden. Beim Abbau der organischen Substanz im Sediment zerfällt das komplizierte Netzwerk der primären Biopolymere in seine Monomerbestandteile, die bei der weiteren Umformung der organischen Fraktion teilweise rekombinieren und zur Bildung einer völlig neuen Art nicht-biologischer Kohlenstoffpolymere („Geopolymere“) führen, die unter dem Begriff „Kerogen“" zusammengefasst werden (Abb. 6).

Kerogen als eine durchgehend veränderte Neubildung auf der Grundlage von organischem Ausgangsmaterial (Durand, 1980) ist ein chemisch inertes (säureunlösliches) polykondensiertes Aggregat von aliphatischen und aromatischen Kohlenwasserstoffen, das als Endprodukt der diagenetischen Umwandlung primärer biogener Substanzen im Sediment anfällt. Als letztliche Derivate ehemals lebender Substanz sind Kerogen und kerogenartige Substanzen (einschließlich ihrer graphitischen Umwandlungsprodukte) eindeutige Zeugnisse früherer Lebensprozesse, zumal sie auch die Kohlenstoffisotopen-Verteilung ihres biologischen Ausgangsmaterials konservieren, die im Vergleich zu nicht-biologischem (Karbonat-)Kohlenstoff durch eine Bevorzugung des leichten Isotops $\left({ }^{12} \mathrm{C}\right)$ gegenüber dem schweren $\left({ }^{13} \mathrm{C}\right)$ gekennzeichnet ist. Gelegentlich führen Kerogenbestandteile auch Relikte organischer Verbindungen von eindeutig biologischer Zuordnung, die ihre molekulare Identität trotz intensiver Umwandlungsprozesse im Sediment offenbar bewahren konnten. Bei solchen „Biomarker“-Molekülen oder „Chemofossilien“ handelt es sich in der Regel um Verbindungen mit einer äußerst widerstandsfähigen Molekülstruktur wie etwa organische Pigmente oder spezielle Kohlenwasserstoffketten. 


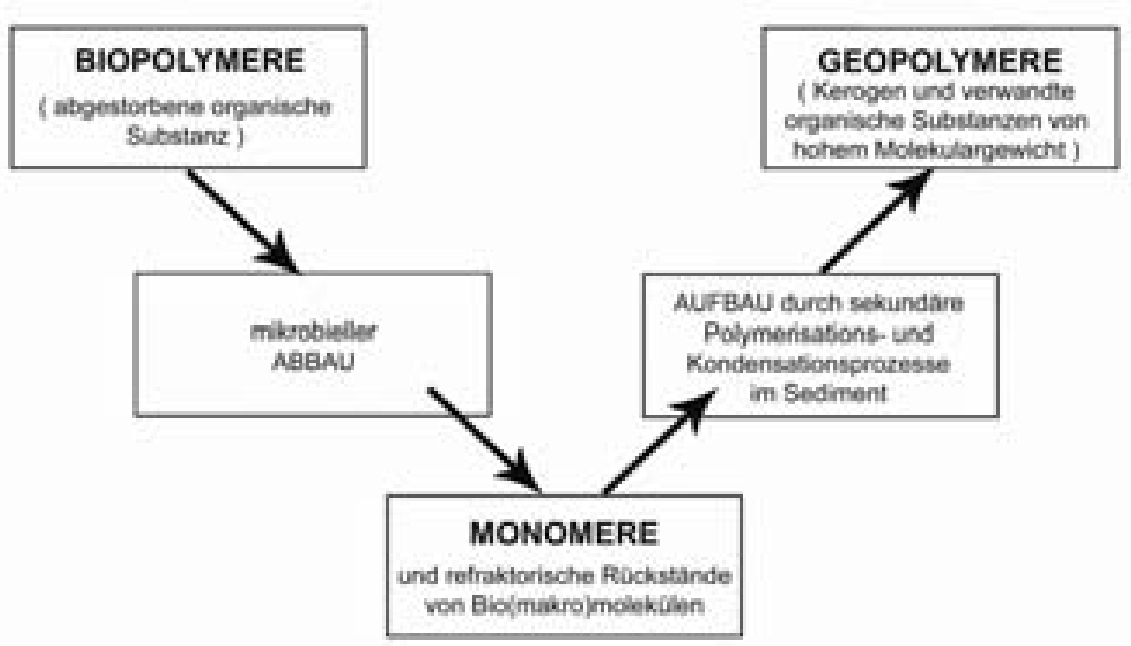

Abb. 6: Bildung von polykondensierten heterogenen „Geopolymeren“(Kerogen) aus den Biopolymeren der primären organischen Substan₹. Der Bildungsweg umfasst einen ,katabolischen' Ast, der Abbauprodukte auf der Ebene von Monomeren liefert, und einen aufsteigenden, anabolischen' Zweig mit einer Rekombination dieser Fragmente im Sediment und nachfolgender Entstebung einer neuen Generation von nicht-biologischen Koblenstoffpolymeren in Form von Kerogen und verwandten Substanzen.

Kerogen als eine durchgehend veränderte Neubildung auf der Grundlage von organischem Ausgangsmaterial (Durand, 1980) ist ein chemisch inertes (säureunlösliches) polykondensiertes Aggregat von aliphatischen und aromatischen Kohlenwasserstoffen, das als Endprodukt der diagenetischen Umwandlung primärer biogener Substanzen im Sediment anfällt. Als letztliche Derivate ehemals lebender Substanz sind Kerogen und kerogenartige Substanzen (einschließlich ihrer graphitischen Umwandlungsprodukte) eindeutige Zeugnisse früherer Lebensprozesse, zumal sie auch die Kohlenstoffisotopen-Verteilung ihres biologischen Ausgangsmaterials konservieren, die im Vergleich zu nicht-biologischem (Karbonat-)Kohlenstoff durch eine Bevorzugung des leichten Isotops $\left({ }^{12} \mathrm{C}\right)$ gegenüber dem schweren $\left({ }^{13} \mathrm{C}\right)$ gekennzeichnet ist. Gelegentlich führen Kerogenbestandteile auch Relikte organischer Verbindungen von eindeutig biologischer Zuordnung, die ihre molekulare Identität trotz intensiver Umwandlungsprozesse im Sediment offenbar bewahren konnten. Bei solchen „Biomarker“-Molekülen oder „Chemofossilien“ handelt es sich in der Regel um Verbindungen mit einer äußerst widerstandsfähigen Molekülstruktur wie etwa organische Pigmente oder spezielle Kohlenwasserstoffketten.

Im Folgenden sollen einige ausgewählte Aspekte der biogeochemischen Überlieferung etwas ausführlicher behandelt werden. 


\section{Sedimentärer organischer Kohlenstoff als Zeugnis vergangenen Lebens}

Wie Abb. 1 zeigt, ist organischer Kohlenstoff $\left(\mathrm{C}_{\text {org }}\right)$ in Form von Kerogen und seinen graphitischen Umwandlungsprodukten ein gängiger Bestandteil irdischer Sedimente seit dem Einsetzen der Überlieferung vor 3,8 Mrd. Jahren. Der $\mathrm{C}_{\mathrm{org}^{-}}{ }^{-}$ Gehalt des durchschnittlichen Sedimentgesteins liegt dabei im Mittel zwischen 0,5 und 0,6 \% (Ronov et al., 1990). Bei einer irdischen Sedimentmasse von etwa $2,4 \times 10^{24} \mathrm{~g}$ würde das bedeuten, dass im Laufe der Erdgeschichte zwischen 1,2 und 1,4 x $10^{22} \mathrm{~g} \mathrm{C}_{\text {org }}$ über ein Leck im biologischen Kohlenstoffzyklus in die irdische Gesteinshülle überstellt worden sind, was Kerogen zur häufigsten Form von organischem Material auf unserem Planeten macht. Tongesteine wie Schiefer zeigen dabei die höchsten $\mathrm{C}_{\mathrm{org}}$-Gehalte (>10\% in „Schwarzschiefern“), während Sandsteine nur äußerst geringe Mengen von organischem Kohlenstoff führen. Die leichtflüchtigen Komponenten der organischen Substanz destillierten beim Reifungsprozess der Kerogenbestandteile weitgehend ab und sammeln sich in Form von Erdöl und gasförmigen Kohlenwasserstoffen in geeigneten Speichergesteinen.

Systematische Untersuchungen an phanerozoischen Sedimenten (Ronov et al., 1990) haben gezeigt, dass die durchschnittlichen $C_{\text {org-Gehalte solcher Gesteine in }}$ den letzten 500 Millionen Jahren nur mäßig um einen Mittelwert von etwa $0,5 \%$ geschwankt haben. Obwohl die Datendichte mit zunehmendem geologischem Alter dramatisch abnimmt, deuten die bisherigen Befunde an, dass sich die entsprechenden Gehalte präkambrischer (proterozoischer und archaischer) Sedimente kaum von denen jüngerer Formationen unterscheiden und insbesondere nicht wesentlich geringer sind (Schidlowski, 1982; Hayes et al., 1983; Strauss \& Moore, 1992). Im Falle der 3,8 Mrd. Jahre alten Isua-Metasedimente erbrachte einen Messreihe sogar ein Mittel von > 0,6\% für die überwiegend graphitische Kohlenstoffkomponente (Schidlowski, 1982).

Obwohl zunächst etwas erstaunlich, müssten die überraschend „modernen“ organischen Kohlenstoffgehalte der ältesten irdischen Sedimente keinesfalls ungewöhnlich sein. Wir wissen heute, dass Mikrobengesellschaften zu den produktivsten Ökosystemen der irdischen Biosphäre zählen und dass bereits benthonische Prokaryonten in der Lage sind, beeindruckend hohe Primärproduktionsraten in der Größenordnung von 8-12 g Corg $/ \mathrm{m}^{2}$ x Tag zu unterhalten (Krumbein \& Cohen, 1977). Weiterhin gilt als sicher, dass insbesondere das frühe Präkambrium das „Goldene Zeitalter“ prokaryotischer Ökosysteme war. Wenn eine derartig hohe Primärproduktion schon von photoautotrophen Mikroorganismen auf der Grundlage einer prokaryotischen Zellorganisation erzielt werden kann, dann dürfte die weitere Evolution der Photosynthese die globale Produktion von Biomasse (und damit indirekt auch den $\mathrm{C}_{\mathrm{org}}-\mathrm{Fluß}$ ins Sediment) quantitativ nur unbedeutend beeinflusst haben. Wir können deshalb annehmen, dass die Produktion von Biomasse bereits in der frühen Erdgeschichte (wie auch heute) weitgehend nährstofflimitiert war. Die frühen mikrobiellen Ökosysteme vermehrten sich dabei 
ungehemmt unter voller Ausnutzung der verfügbaren Lebensräume bis zu einem Zustand globaler „biotischer Saturation“, der sich nach der Erschöpfung kritischer Nährstoffe (wie Phosphor und Stickstoff) zwangsläufig einstellte.

\section{${ }^{13} \mathrm{C} /{ }^{12} \mathrm{C}$ in fossilem Kohlenstoff: Isotopensignatur der Photosynthese}

Kohlenstoff besteht im Wesentlichen aus einer Mischung von zwei stabilen Isotopen, ${ }^{12} \mathrm{C}$ und ${ }^{13} \mathrm{C}$ (ein drittes kurzlebiges radioaktives Nuklid, ${ }^{14} \mathrm{C}$, existiert nur in Spuren). Es hat sich gezeigt, dass alle Umwandlungen dieses Elements innerhalb der geochemischen Stoffkreisläufe zu merklichen Verschiebungen der Isotopenverhältnisse führen. Diese beruhen auf thermodynamischen oder kinetischen Fraktionierungseffekten bei der jeweiligen Reaktion und sind letztlich der Grund dafür, dass sich die Reaktionsprodukte in ihrem ${ }^{13} \mathrm{C} /{ }^{12} \mathrm{C}$-Verhältnis von den Ausgangssubstanzen unterscheiden. Die größten Isotopenverschiebungen treten bei der Umwandlung von anorganischem in organischen Kohlenstoff während der Photosynthese auf, d. h. bei der Assimilation von $\mathrm{CO}_{2}$ und Bikarbonat $\left(\mathrm{HCO}_{3}^{-}\right.$) durch Pflanzen und photoautotrophe Mikroorganismen (siehe Gl. 1). Grundsätzlich sind alle Wege autotropher Kohlenstoff-Fixierung mit Fraktionierungen der stabilen Kohlenstoffisotope verbunden, die zu einer deutlichen Anreicherung des leichten Isotops $\left({ }^{12} \mathrm{C}\right)$ in der neu gebildeten Biomasse führen.

Die quantitativ wichtigsten Isotopeneffekte treten dabei an zwei Stellen der assimilatorischen Reaktionsfolge auf, nämlich (1) bei der Aufnahme von externem $\mathrm{CO}_{2}$ und dessen Diffusion zu den photosynthetisch aktiven Zentren innerhalb der Zelle, und (2) bei der ersten $\mathrm{CO}_{2}$-fixierenden Carboxylierungsreaktion, bei der das zugeführte $\mathrm{CO}_{2}$ in die Carboxylgruppe $(\mathrm{COOH})$ einer organischen Verbindung (meist Phosphoglycerinsäure oder Oxalessigsäure) überführt wird. Die für die biologische Kohlenstoffisotopenfraktionierung verantwortlichen Prozesse lassen sich deshalb mit guter Annäherung mit folgendem Zweistufenmodell (Gl. 2) beschreiben:

$$
\mathrm{CO}_{2(\mathrm{e})} \stackrel{k_{1}}{\rightleftarrows} \mathrm{CO}_{2} \underset{k_{2}(\mathrm{i})}{\stackrel{k_{3}}{\rightleftarrows}} \mathrm{COOH}
$$

Hier stehen $\mathrm{CO}_{2(\mathrm{e})}$ und $\mathrm{CO}_{2(\mathrm{i})}$ für externes und internes (intrazellulares) $\mathrm{CO}_{2}$, und $\mathrm{k}_{1}-\mathrm{k}_{4}$ sind die Ratenkonstanten der assimilatorischen und gegenläufigen (dissimilatorischen) Reaktionen. Die bei den jeweiligen Einzelprozessen auftretenden Fraktionierungen führen insgesamt zu einer charakteristischen Anreicherung von „leichtem“ Kohlenstoff $\left({ }^{12} \mathrm{C}\right)$ in allen biosynthetisch entstandenen Substanzen verglichen mit dem Kohlendioxid des irdischen Umwelt-Pools. Während der initiale Diffusionsschritt in G1. 2 nur durch eine geringe Isotopenverschiebung zugunsten von ${ }^{12} \mathrm{C}$ gekennzeichnet ist, treten bei der nachfolgenden ersten irreversiblen $\mathrm{CO}_{2-}$ 
fixierenden Carboxylierungsreaktion beträchtliche Fraktionierungen auf, die aus den isotopenselektierenden Eigenschaften der beteiligten Enzyme (meist Ribulose-1,5-Bisphosphat-Carboxylase) resultieren (Park \& Epstein, 1960; O’Leary, 1981; Schidlowski et al., 1983). Diese Reaktion besorgt die Überführung von anorganischem Kohlenstoff in die Carboxylgruppe (COOH) einer organischen Säure, die dann in den weiteren Stoffwechsel des Organismus eintritt. Da die biologische Kohlenstoffassimilation überwiegend enzymatisch gesteuert wird und Lebewesen dynamische Zustände darstellen, die ständigen (und oft sehr raschen) Zyklen von Aufbau (Anabolismus) und Abbau (Katabolismus) unterliegen, ist allgemein akzeptiert, dass bei biologischen Isotopenfraktionierungen kinetische gegenüber thermodynamischen Effekten eindeutig überwiegen.

Quantitativ werden diese Isotopenverschiebungen als sog. „ $\delta$-Werte“ angegeben, die die Promille-Abweichung im ${ }^{13} \mathrm{C} /{ }^{12} \mathrm{C}$-Verhältnis der untersuchten Substanz oder Probe (sa) von einem konventionellen Standard (st) anzeigen, d. h. $\delta^{13} \mathrm{C}=\left[\left({ }^{13} \mathrm{C} /{ }^{12} \mathrm{C}\right)_{\mathrm{sa}} /\left({ }^{13} \mathrm{C} /{ }^{12} \mathrm{C}\right)_{\mathrm{st}}-1\right] \times 1000$ (Promille). Positive $\delta^{13} \mathrm{C}$-Werte bedeuten demnach eine Anreicherung von schwerem Kohlenstoff $\left({ }^{13} \mathrm{C}\right)$ in der jeweiligen Probe gegenüber dem Standard, während negative Werte eine entsprechende Abreicherung (und damit eine Zunahme von ${ }^{12} \mathrm{C}$ ) signalisieren. Der Standard, der die Null-Promille-Linie auf der $\delta$-Skala definiert, ist die Karbonatsubstanz eines fossilen Cephalopoden (Belemnitella americana) aus der Peedee-Formation (PDBelemnit, PDB) mit ${ }^{12} \mathrm{C} /{ }^{13} \mathrm{C}=88,99$.

$\mathrm{Da}$ alle Wege biologischer Kohlenstoff-Fixierung zu einer relativen Anreicherung von leichtem Kohlenstoff $\left.{ }^{12} \mathrm{C}\right)$ gegenüber dem anorganischen Ausgangsmaterial $\left(\mathrm{CO}_{2}\right.$ und $\left.\mathrm{HCO}_{3}^{-}\right)$führen, liegen die $\delta^{13} \mathrm{C}$-Werte organischer Substanzen durchgehend im negativen Bereich. Abb. 7 zeigt die Isotopenverteilungsmuster der wichtigsten Gruppen höherer Pflanzen, eukaryotischer Algen sowie photosynthetischer und chemosynthetischer Bakterien. Die teilweise recht unterschiedlichen Streubreiten der einzelnen Gruppen resultieren aus dem abgestuften $\mathrm{Zu}$ sammenspiel der in Gl. 2 dargestellten isotopendiskriminierenden Prozesse sowie aus der Beteiligung verschiedener $\mathrm{CO}_{2}$-fixierender Enzyme mit sehr unterschiedlichem isotopischem Selektionspotential (wie etwa im Falle der divergierenden Verteilungsmuster von C3- und C4-Pflanzen). Je nachdem, welcher der isotopendiskriminierenden Teilprozesse die Ratenkontrolle über den Gesamtprozess erlangt, oder welches spezielle Enzym die Carboxylierungsreaktion katalysiert, kann die isotopische Zusammensetzung pflanzlicher und bakterieller Biomasse beträchtlich schwanken (für eine ausführliche Diskussion vgl. Schidlowski et al., 1983 und Schidlowski \& Aharon, 1992). Versucht man eine Wichtung der in Abb. 7 zusammengestellten $\delta^{13} \mathrm{C}$-Verteilungsmuster nach dem Anteil der jeweiligen Primärproduzenten an der globalen Biomasse, dann dürfte der $\delta^{13} \mathrm{C}$-Wert der durchschnittlichen irdischen Biomasse verglichen mit dem für ozeanisches Bikarbonat (dem größten anorganischen Kohlenstoffreservoir an der Erdoberfläche) um 20-30\% in den negativen Bereich verschoben worden sein. Es sollte hier ausdrücklich festgehalten werden, dass diese auffällige Anreicherung von ${ }^{12} \mathrm{C}$ in der heutigen Biosphäre zum größten Teil das Resultat der isotopendiskriminie- 
renden Eigenschaften eines einzigen Enzyms ist, nämlich von Ribulose-1,5Bisphosphat (RuBP)-Carboxylase, dem Schlüsselenzym des Calvin-Zyklus, über den der überwiegende Teil des Kohlenstofftransfers von der unbelebten in die belebte Welt abgewickelt wird. Es ist somit keinesfalls überraschend, dass gerade die RuBP-Carboxylase-Reaktion der irdischen Biomasse mit einem $\delta^{13} \mathrm{C}$-Mittel von etwa $-26 \pm 7 \%$ [PDB] ihre spezifische Isotopensignatur aufgeprägt hat.

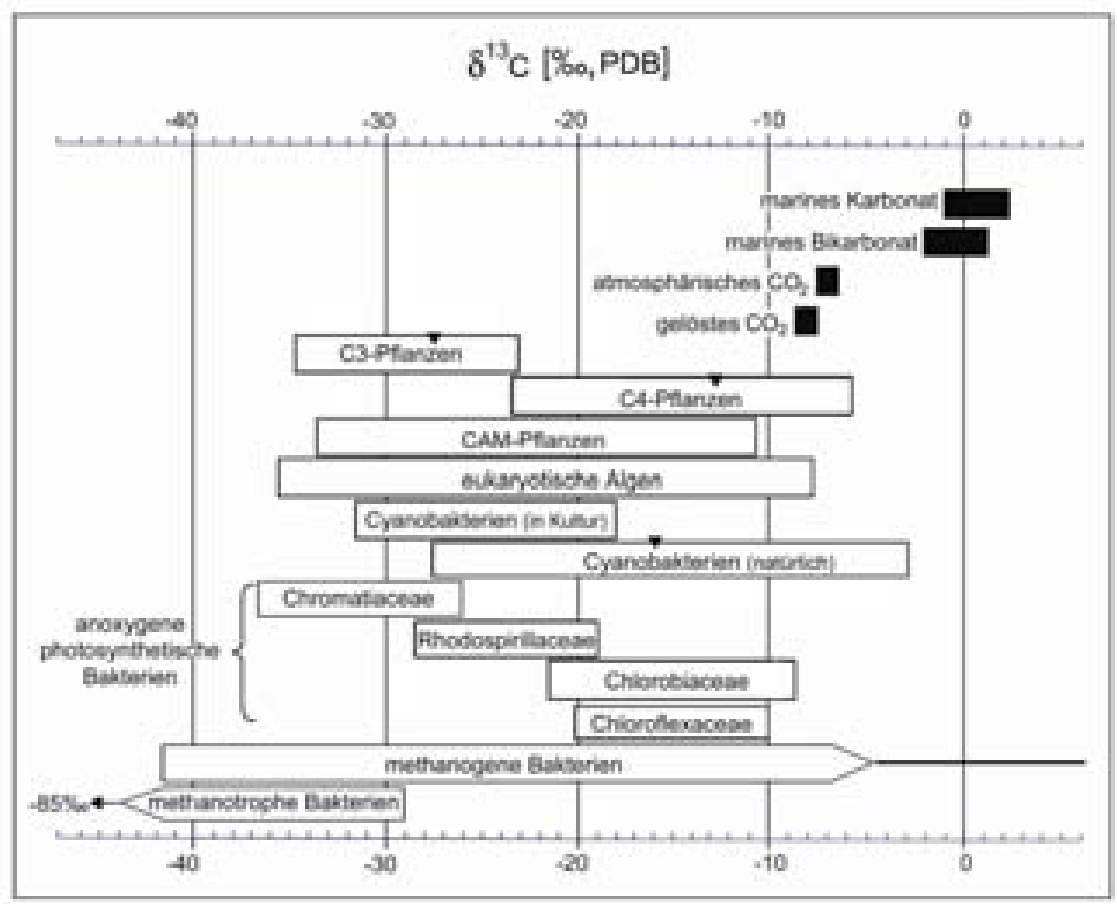

Abb. 7: Streubreiten der $\delta^{13} C$-Werte von Pflanzen und Mikeroorganismen (schattiert) verglichen mit den entsprechenden Werten der drei anorganischen Koblenstoff-Reservoire $\left(\mathrm{CO}_{2}, \mathrm{HCO}_{3}^{-}, \mathrm{CO}_{3}^{2-}\right)$ der irdischen Umwelt (schwar:). Die durchgängig negativen $\delta^{13} \mathrm{C}$-Verteilungsspektren des biologischen Materials zeigen eine deutliche Anreicherung von leichtem Koblenstoff $\left({ }^{2} C\right)$ im Vergleich zum anorganischen Lieferreservoir (überwiegend $\mathrm{CO}_{2}$ ) an.

Für die Dokumentation vergangenen Lebens ist nun ganz wichtig, dass die auf diese Weise erzeugte Isotopendifferenz zwischen biogenem (organischem) Kohlenstoff und dem anorganischen Bikarbonat-Karbonat-Reservoir der Erdoberfläche im Wesentlichen erhalten bleibt, wenn beide Kohlenstoff-Varianten in den Stoffbestand neugebildeter Sedimente eintreten. Wie Abb. 8 zeigt, werden die isotopischen Streubreiten sowohl der heutigen Biomasse als auch von marinem Karbonat und Bikarbonat mit geringen Abstrichen in die sedimentäre Überlieferung bis zurück zu 3,5 oder sogar 3,8 Mrd. Jahren transkribiert. Das bedeutet, dass organischer und karbonatischer Kohlenstoff über die gesamte Erdgeschichte ohne 
nennenswerte Veränderungen in ihrer isotopischen Zusammensetzung von der Erdoberfläche in die Kruste überführt worden sind. So widerspiegelt etwa die Bandbreite der $\delta^{13} \mathrm{C}_{\text {org }}$-Werte heutiger mariner Sedimente getreulich die Verteilungsmuster der irdischen Primärproduzenten, wobei lediglich die (quantitativ unbedeutenden) Extreme gekappt sind (Abb. 8, rechte Box). Daraus ergibt sich, dass die diagenetische Umwandlung der organischen Substanz im Sediment nur zu geringen Isotopenverschiebungen führt (meist $<3 \%$, vgl. Hayes et al., 1983 und Schidlowski \& Aharon, 1993), wobei diese Schwankungen in der Regel im breiten Streufeld der ursprünglichen Werte untergehen. Somit wird der bei der Photosynthese auftretende Isotopeneffekt fast unverändert von der Biosphäre in den geologischen Teil der irdischen Kohlenstoffkreislaufs überliefert, was uns wiederum die Möglichkeit eröffnet, den diesem Signal zugrunde liegenden Prozess weit in die geologische Vergangenheit zurückzuverfolgen.

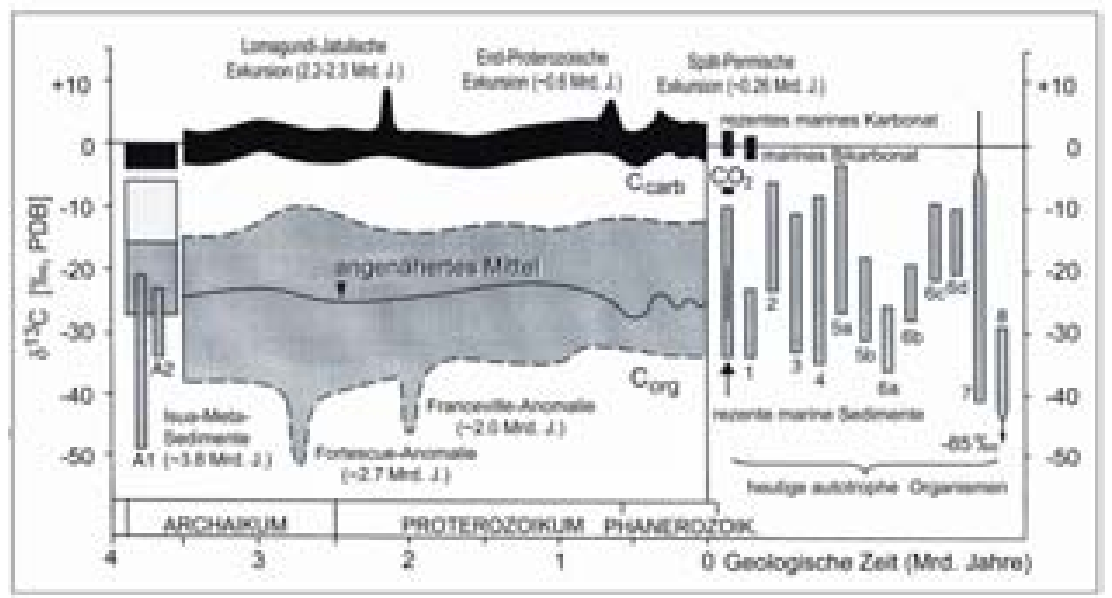

Abb. 8: Isotopen-Altersfunktionen von Karbonatkoblenstoff $\left(C_{\text {carb }}\right)$ und organischem Koblenstoff (Corg) im Vergleich zur isotopischen Zusammensetzung der jeweiligen Vorläufersubstanzen in der heutigen Umwelt (marines Bikarbonat und biologisches Material verschiedener Herkunft wie in Abb. 7, siehe rechte Box). Man beachte, dass die $\delta^{3} C_{\text {org }}$-Verteilung in rezenten marinen Sedimenten die gesamte Streubreite der heutigen Biomasse integriert, wobei $>90 \%$ der Werte auf dem schwarzen Innenband (zwischen -19 und -28\%o) liegen. Das heutige Verteilungsspektrum lässt sich über einen Zeitraum von 3,8 Mrd. Jahren in die Erdgeschichte zurückverfolgen, wobei lediglich die Isua-Werte am Beginn der Überlieferung durch die Gesteinsmetamorphose verschoben sind. In dem versetzten Isua-Segment zeigt der schattierte untere Teil den Streubereich derjenigen $\delta^{13} C_{\text {org }}-W$ erte an, die noch eine biologische Herkunft erkennen lassen, während die Werte im oberen (punktierten) Feld durch den ${ }^{13} \mathrm{C} /{ }^{12} \mathrm{C}$-Austausch bei der Metamorphose in den positiven Bereich verschoben worden sind. Die überlagernden Barren geben die Streubreiten der $\delta^{13} C_{\text {org }}$ Werte für Koblenstoffeinschlüsse in akzessorischen Apatiten der Akilia-Eisensteinformation (A1) und verschiedenen Vorkommen gebänderter Eisensteine aus der Isua-Serie (A2) an. 
Bei diesem Sachverhalt ist eine schlüssige Deutung der in der sedimentären Abfolge gespeicherten Isotopendaten naheliegend. Es scheint eindeutig, dass die auffällige ${ }^{12} \mathrm{C}$-Anreicherung im Datenband für fossilen organischen Kohlenstoff (Abb. 8, linke Box) ein kohärentes Signal (photo)autotropher Kohlenstoff-Fixierung über die Erdgeschichte darstellt. Die in Abb. 8 zusammengefassten Daten zeigen somit den globalen Fraktionierungsprozess zwischen biologischem und nicht-biologischem (karbonatischem) Kohlenstoff in seiner erdgeschichtlichen Dimension, wobei die weitgehende Gleichförmigkeit des Isotopensignals einen extremen Grad von evolutionärem Konservatismus im Mechanismus der Photosynthese als des quantitativ wichtigsten biochemischen Prozesses auf der Erde bezeugt. Tatsächlich lässt sich der Hauptstrom des Bandes für $\delta^{13} \mathrm{C}_{\text {org }}$ in Abb. 8 am sinnvollsten als geochemische Manifestation der isotopendiskriminierenden Eigenschaften eines einzigen Enzyms interpretieren, nämlich von Ribulose-1,5Bisphosphat (RuBP)-Carboxylase, dem Schlüsselenzym des Calvin-Zyklus.

Wir wissen heute, dass die Hauptmasse des Kohlenstofftransfers von der unbelebten in die belebte Welt über die RuBP-Carboxylase-Reaktion erfolgt, die das assimilierte $\mathrm{CO}_{2}$ als Phosphoglycerinsäure (eine sog. „C3“-Verbindung mit einem Skelett von 3 Kohlenstoffatomen) direkt in den Calvin-Zyklus einspeist. Die Mehrzahl der autotrophen Mikroorganismen und alle grünen Pflanzen bedienen sich dieses Weges der $\mathrm{CO}_{2}$-Assimilation (höhere Pflanzen, die ihn ausschließlich benutzen, werden als C3-Pflanzen bezeichnet). Als Endresultat trägt die Hauptmenge der irdischen Biomasse (rezent und fossil) die Isotopensignatur der C3Photosynthese mit einem globalen $\delta^{13} \mathrm{C}_{\text {org }}$-Mittel von etwa $-26 \pm 7 \%$.

Gelegentlich auftretende negative Extreme des Hauptstroms der $\delta^{13} \mathrm{C}_{\text {org }}$ Altersfunktion (Abb. 8) sind auf die frühe (präkambrische) Erdgeschichte beschränkt. Da eine derart auffällige Anreicherung von ${ }^{12} \mathrm{C}$ nur von methanotrophen Bakterien bekannt ist, dürften sich diese isotopisch leichten Kerogen-Varianten von Biomasse herleiten, die ganz oder weitgehend durch Assimilation von Methan $\left(\mathrm{CH}_{4}\right)$ entstanden ist. Obwohl diese $\delta^{13} \mathrm{C}_{\text {org }}$-Minima auf den ersten Blick als unbedeutende Randphänomene des globalen Kohlenstoffkreislaufs erscheinen könnten, ergibt sich andererseits aus Abb. 8, dass sie offenbar Ausstülpungen einer im frühen Präkambrium generell erniedrigten Untergrenze für $\delta^{13} C_{o r g}$ sind. Die auf diese Weise angedeutete relative Zunahme von isotopisch leichten ${ }^{12} \mathrm{C}$-angereicherten) Kerogenkomponenten könnte ein Hinweis dafür sein, dass Methan bei den Kohlenstoffumsätzen im biogeochemischen Zyklus der frühen Erdgeschichte eine weit bedeutendere Rolle gespielt hat als in der nachfolgenden Zeit (Hayes, 1994). 


\section{${ }^{13} \mathrm{C} /{ }^{12} \mathrm{C}$ in den ältesten Sedimenten: Probleme der metamorphen Überlieferung}

Die auffälligste Diskontinuität in der irdischen Kohlenstoffisotopen-Alterskurve ist der Bruch zwischen den Metasedimenten der Isua-Serie von West-Grönland mit einem Alter von 3,6-3,8 Mrd. Jahren (Moorbath et al., 1973) und der jüngeren Überlieferung (Abb. 8). Wie im Folgenden gezeigt wird, ist diese Diskontinuität beiden $\delta^{13} \mathrm{C}$-Altersfunktionen $\left(\mathrm{C}_{\text {arb }}\right.$ und $\left.\mathrm{C}_{\mathrm{org}}\right)$ jedoch sekundär durch die Gesteinsmetamorphose aufgeprägt worden. Wie wir heute wissen, gehen die ältesten unveränderten Sedimente nur bis zu etwa 3,5 Mrd. Jahren zurück. Noch ältere Gesteine sind äußerst selten und in der Regel stark metamorph verändert. Dieser früheste Teil der geologischen Überlieferung wird im Wesentlichen durch die Metasedimente der Isua-Serie repräsentiert, die außer einem selektiven Stoffaustausch durch Metasomatose noch einer Hochtemperaturmetamorphose vom Typ der Amphibolit-Fazies $\left(450-650{ }^{\circ} \mathrm{C}\right.$ ) unterworfen waren (Rosing et al., 1996; Appel et al., 1998).

Da die in den Isua-Metasedimenten beobachteten Isotopenverschiebungen den thermodynamisch kalkulierten Verschiebungsbeträgen entsprechen, wie man sie bei einer Neueinstellung der Isotopengleichgewichte zwischen organischem $\left(\mathrm{C}_{\text {org }}\right)$ und karbonatischem Kohlenstoff $\left(\mathrm{C}_{\text {carb }}\right)$ unter Hochtemperaturbedingungen erwarten müsste, ist kaum ein Zweifel darüber möglich, dass diese Verschiebungen dem Gestein nachträglich aufgeprägt worden sind. Sowohl die verfügbaren thermodynamischen Daten über den ${ }^{13} \mathrm{C} /{ }^{12} \mathrm{C}$-Austausch zwischen $\mathrm{C}_{\text {org }}$ und $\mathrm{C}_{\text {carb }}$ als Funktion steigenden Metamorphosegrades als auch Geländebefunde von geologisch jüngeren metamorphen Terrains (Abb. 9) lassen es sicher erscheinen, dass die Isua-Werte sekundär versetzt sind und die „normalen“ langfristigen Isotopentrends der jüngeren Erdgeschichte ursprünglich bis 3,8 Mrd. Jahren durchgehalten hatten (Schidlowski et al., 1979, 1983; Schidlowski, 1988, 2001). Voraussetzung für solche Verschiebungen der Isotopengleichgewichte ist immer eine vorhergehende Hochtemperaturreaktion von Karbonat mit der Silikatfraktion des Gesteins unter Neubildung von Kalksilikaten, die zu einer massiven Dekarbonatisierung der primären Karbonatkomponente und einer entsprechenden Freisetzung von Kohlendioxid führt. Ein Beispiel dafür ist die Reaktion von Dolomit mit Quarz unter Neubildung von Tremolit und Kalziumkarbonat:

$5 \mathrm{CaMg}\left(\mathrm{CO}_{3}\right)_{2}+8 \mathrm{SiO}_{2}+\mathrm{H}_{2} \mathrm{O} \rightarrow \mathrm{Ca}_{2} \mathrm{Mg}_{5}(\mathrm{OH})_{2} \mathrm{Si}_{8} \mathrm{O}_{22}+3 \mathrm{CaCO}_{3}+7 \mathrm{CO}_{2}(\mathrm{Gl} .3)$

Die bei dieser Reaktion entbundene mobile $\mathrm{CO}_{2}$-Phase fungiert danach als Medium des Isotopenaustausches zwischen Kerogen und Karbonat. Kalkulierte Fraktionierungsfaktoren für die Isotopenaustauschreaktionen von Gl. 3 zeigen, dass das entgaste $\mathrm{CO}_{2}$ gegenüber dem Ausgangskarbonat an schwerem Kohlenstoff $\left.{ }^{13} \mathrm{C}\right)$ angereichert und dass das bei der Reaktion anfallende Restkarbonat $\left(\mathrm{CaCO}_{3}\right)$ entsprechend an ${ }^{13} \mathrm{C}$ abgereichert (also isotopisch leichter) ist (Bottinga, 
1969). Die Einstellung eines neuen Isotopengleichgewichtes zwischen dem freigesetzten „schweren“ $\mathrm{CO}_{2}$ und den ,leichten“ Kerogenbestandteilen des umgebenden Gesteins führt in der Folge dazu, dass die Unterschiede im ${ }^{13} \mathrm{C} /{ }^{12} \mathrm{C}-\mathrm{Ver}$ hältnis zwischen organischem Kohlenstoff und Karbonat mit ansteigender Temperatur der Metamorphose (Abb. 9) immer geringer werden. Abb. 8 zeigt die Auswirkung dieses Prozesses auf die Isua-Serie in Form einer auffällig geschrumpften Differenz zwischen den $\delta^{13} \mathrm{C}$-Werten von $\mathrm{C}_{\text {carb }}$ und $\mathrm{C}_{\mathrm{org}}$, wobei das Karbonat etwas „leichter“ und die (überwiegend graphitisierte) organische Komponente signifikant ,,schwerer“ geworden ist.

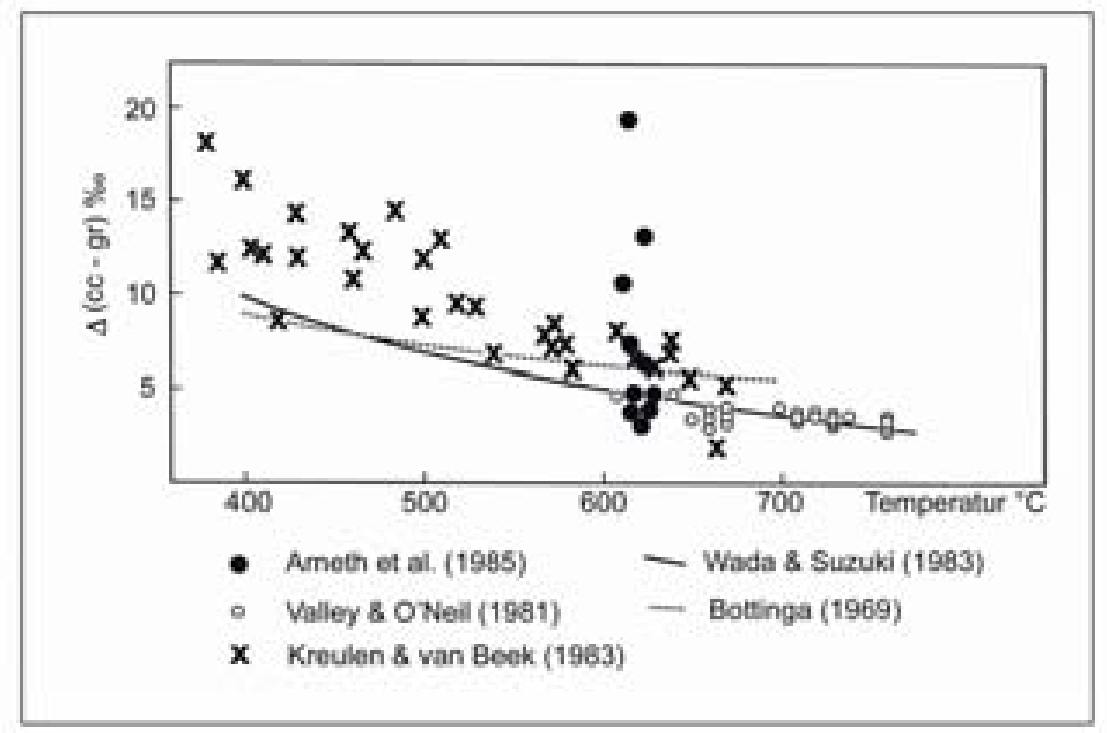

Abb. 9: Abnahme der Isotopenfraktionierung zwischen organischem und karbonatischem Koblenstoff als Funktion ansteigender Temperaturen bei der Gesteinsmetamorphose. In metamorphen Gesteinen wird diese Fraktionierung als Differenz, zwischen den $\delta^{3} \mathrm{C}$-Werten von koexistierendem Kalzit (cc) und Graphit (gr) angegeben, d. h. $\Delta(c c-g r)=\delta^{13} C_{c c}-\delta^{3} C_{g r}$. Die von verschiedenen Autoren mitgeteilten Daten von ausgewählten metamorphen Terrains strenen um die thermodynamisch kalkulierte Funktion der Isotopengleichgewichte von Bottinga (1969) und eine empirische Fraktionierungskurve von Wada \& Suquki (1983), deren Temperaturverlauf an Dolomit-Kalzit-Solvustemperaturen geeicht ist. Man beachte die zunehmende Verengung des Streufeldes mit ansteigender Temperatur, obwobl einzelne abweichende Werte im T-Bereich 600-650 ${ }^{\circ} \mathrm{C}$ belegen, dass die Neueinstellung eines isotopischen Gleichgewichts selbst noch in der oberen Amphibolit-Fazies kinetisch retardiert sein kann. 
Somit scheint der Schluss zwingend, dass die langfristigen Trends beider Isotopenaltersfunktionen ursprünglich bis zum Einsetzen der sedimentären Überlieferung vor 3,8 Mrd. Jahren durchgehalten hatten. Es sollte in diesem $\mathrm{Zu}$ sammenhang auch festgehalten werden, dass der ${ }^{13} \mathrm{C} /{ }^{12} \mathrm{C}$-Austausch zwischen $\mathrm{C}_{\text {org }}$ und $\mathrm{C}_{\text {carb }}$ bei der Metamorphose tendenziell stets zu einer Erhöhung der $\delta^{13} \mathrm{C}$ Werte der organischen Komponente (Kerogen und Graphit) führt, und dass deshalb die tiefsten angetroffenen Werte immer die ursprünglicheren und am wenigsten veränderten sind. Nachdem bereits bei den frühen Untersuchungen der Isua-Serie $\delta^{13} \mathrm{C}$-Werte zwischen -22 und $-28 \%$ für die graphitische Substanz gefunden wurden (Schidlowski et al., 1979, 1983; Hayes et al., 1983), war es eindeutig, dass die ältesten irdischen Sedimente schon organische Komponenten mit der isotopischen Zusammensetzung von biologischem Material enthielten.

Weiterhin haben unlängst Mojzsis et al. (1996) eine Serie von Isotopenanalysen an Einschlüssen kohliger Substanz in mikroskopischen Apatitkörnchen der Akilia-Eisenstein-Formation des erweiterten Isua-Gebietes durchgeführt. Die Messungen an den winzigen Kohlenstoffpartikeln wurden mit Hilfe moderner Mikroanalytik (Ionen-Mikrosonde) gemacht und ergaben Werte zwischen -21 und $-49 \%$ mit einem gewichteten Mittel von $-37 \pm 3 \%$. Auch wenn die Präzision der Einzelmessung bei dieser Methode nur bei $\pm 5 \%$ liegt, fällt die Spannbreite der Werte eindeutig in den biologischen Bereich, wobei die auffällige Verschiebung zu negativen Werten auf eine methanotrophe Herkunft der ursprünglichen Biomasse deutet. In diesem Falle könnte es sich durchaus um primäre Werte handeln, die wegen des Auftretens der kohligen Substanz in einem karbonatfreien Gestein und ihrer weitgehenden Isolierung durch eine umhüllende Mineralphase keinem nachträglichen Isotopenaustausch unterworfen waren.

Trotz verbleibender Unsicherheiten in einigen Details (vgl. Van Zuilen et al., 2002; Fedo \& Whitehouse, 2002) zwingen die vorliegenden Daten und erkannten Zusammenhänge in ihrer Gesamtheit zu dem Schluss, dass die Kohlenstoffisotopenüberlieferung der frühen ( $>$ 3,5 Mrd. Jahre) Erdgeschichte bereits eindeutige Hinweise auf die Existenz von Leben auf unserem Planeten gibt. Um eine biologische Interpretation des ältesten Segments der irdischen Kohlenstoffisotopen-Alterskurve zu widerlegen, müssten wir einen nicht-biologischen Prozess von globalem Ausmaß postulieren, der die Isotopenfraktionierung bei der biologischen Kohlenstoff-Fixierung (speziell der Photosynthese) in Richtung und GröBenordnung mit bemerkenswerter Präzision kopiert. Da ein solcher alternativer Prozess nicht bekannt ist und ein frühes Auftreten von Leben auf der Erde zudem durch andere Befunde bezeugt wird, würde die vorliegende Evidenz in der Gesamtbilanz entschieden die These stützen, dass biologische KohlenstoffisotopenFraktionierungen seit dem Beginn der sedimentären Überlieferung ständig auf der Erde stattgefunden haben. Entsprechend lässt sich der Hauptstrom der $\delta^{13} \mathrm{C}_{\text {org- }}$ Altersfunktion von Abb. 8 am sinnvollsten als isotopische Kennlinie autotropher Kohlenstoff-Fixierung über 3,8 Mrd. Jahre Erdgeschichte interpretieren. 


\section{Molekulare Bioindikatoren („Chemofossilien“)}

Einige Verbindungen eindeutig biologischer Herkunft (meist robuste Einzelmoleküle oder spezifische Molekülbruchstücke) waren offenbar in der Lage, ihre strukturelle und stereochemische Identität bei der Umwandlung der organischen Substanz im Sediment (vgl. Abb. 6) in den Grundzügen zu bewahren. Als chemische Gegenstücke zu morphologischen Lebensresten kann man sie als „Chemofossilien“ (Eglinton \& Calvin, 1967) bezeichnen, die durch ihre Anwesenheit im Gestein bezeugen, dass die für ihre Entstehung verantwortlichen enzymatischen Prozesse schon über Milliarden von Jahren scheinbar unverändert gearbeitet haben.

Organische Pigmente wie Porphyrine waren die ersten molekularen Fossilien, die seit den Pionierarbeiten von Treibs (1934) aus Sedimentgesteinen und Erdölen verschiedenen Alters bekannt geworden sind. Die für Porphyrine charakteristische Molekulararchitektur (ein Tetrapyrrol-Ring mit einem zentralen Metalleinschluss) liegt sowohl der Chlorophyll- als auch der Häminstruktur zugrunde, die sich im Wesentlichen durch die unterschiedliche Besetzung der Metallposition mit $\mathrm{Mg}$ bzw. Fe unterscheiden. Die Deutung fossiler Porphyrine als Chlorophyll- und Häminderivate war somit zwangsläufig. Die seitliche Phytylkette des Chlorophyllmoleküls wird außerdem bei der Diagenese in zwei verschiedene Isoprenoide (Phytan und Pristan) umgewandelt, die als separate Kohlenwasserstoffketten gleichfalls wichtige chemische „Biomarker“ darstellen.

Ein weiteres prominentes Biomarker-Molekül ist Cholesterin, eine Sterolverbindung, die in allen höheren (eukaryotischen) Lebensformen weit verbreitet ist. Das Cholesterinmolekül besitzt ein hochorganisiertes Skelett von Kohlenstoffatomen mit einer spezifischen Stereochemie, die u. a. durch acht chirale Zentren gekennzeichnet ist. Diese verleihen dem Molekül einen hohen Grad von intramolekularer Komplexität, die noch dadurch verstärkt wird, dass jedes dieser Zentren besondere Präferenzen für das ${ }^{13} \mathrm{C} /{ }^{12} \mathrm{C}$-Verhältnis bei der Besetzung der zugehörigen C-Positionen ausübt. Diese Eigenschaften machen Cholesterin zu einem eindeutig zu identifizierenden organischen Relikt im Sediment und damit zu einem aussagekräftigen molekularen Dokument vergangenen Lebens. In diesem Zusammenhang ist erwähnenswert, dass spezifische Sterolabkömmlinge (Sterane mit Skeletten von 28-30 C-Atomen) kürzlich in 2,7 Mrd. Jahre alten Schiefern der Hamersley-Formation des australischen Pilbara-Kratons nachgewiesen worden sind (Brocks et al., 1999). Da Sterolvorläufer von Steranen mit einer derartig hohen Anzahl von C-Atomen bisher noch nie in niederen (prokaryotischen) Organismen angetroffen wurden, liegt der Schluss nahe, dass die Evolution der eukaryotischen Zelle wesentlich früher erfolgt ist als bisher allgemein angenommen wurde. Aus den gleichen Hamersley-Sedimenten wurde auch eine Serie von Cyanobakterien-spezifischen molekularen Fossilien ( $2 \alpha$-Methylhopane) isoliert. Die Gruppe der 2-Methylhopanoide umfasst eine Anzahl von pentazyklischen Triterpanen (Kohlenwasserstoffen), die als Abbauprodukte spezifischer 
Lipide anfallen, die in großem Maßstab in den Membranen von Cyanobakterien synthetisiert werden (Summons et al., 1999). Diese Befunde dürften das frühe Auftreten von Cyanobakterien in der Erdgeschichte weiter abstützen, das auch durch andere Evidenz belegt ist.

\section{Zusammenfassung und Ausblick}

Die aus der frühen Erdgeschichte überlieferten paläontologischen und biogeochemischen Befunde erlauben eindeutig den Schluss, dass mikrobielles (prokaryotisches und archaeoprokaryotisches) Leben seit mindestens 3,5 Mrd. Jahren fest auf unserem Planeten etabliert war. Berücksichtigt man die reliktische Evidenz aus metamorph überprägten älteren Gesteinen wie der Isua-Metasedimente von WestGrönland, dann kann man sogar mit guten Gründen für einen Beginn von Lebensprozessen vor 3,8 Mrd. Jahren plädieren. Insbesondere die Isotopen-Altersfunktion des sedimentären organischen Kohlenstoffs mit ihrer durchgängigen Bevorzugung von ${ }^{12} \mathrm{C}$ (Abb. 8) lässt sich am sinnvollsten als isotopische Kennlinie biologischer Kohlenstoff-Fixierung über 3,8 Mrd. Jahre Erdgeschichte interpretieren. Bei einem Alter der Erde von 4,56 Mrd. Jahren würde das bedeuten, dass sich die frühe Evolution des Lebens bis zur Herausbildung der prokaryontischen Zellorganisation innerhalb von nur 700 Millionen Jahren vollzogen haben muss. Da der früheste „hadeische“ Zeitabschnitt der Erdgeschichte (vgl. Abb. 1) nicht durch Sedimentgesteine als potentielle Informationsträger belegt ist, verfügen wir über keinerlei empirische Evidenz, sodass sich hier noch eine weites Feld für Spekulationen eröffnet, die selbst eine außerirdische Herkunft der hypothetischen Ahnform des Lebens, des sog. „Progenoten“, nicht ausschließen.

Speziell die jüngsten Befunde der „molekularen Phylogenese“, die sich auf einen Vergleich ausgewählter Nukleinsäuresequenzen der verschiedenen Organismenstämme gründen, lassen es zwingend erscheinen, dass alles irdische Leben von einem derartigen Progenoten abstammt (Kandler, 1993). Alle weitere Diversifizierung dürfte sich danach in einem evolutionären Kontinuum vollzogen haben, in dem eine Evolution von „darwinischem“ Typ auf der Grundlage eines DNAgegründeten genetischen Programms ablief. Der molekulare Stammbaum des irdischen Lebens erlaubt die Definition von drei Großreichen (Archaeoprokaryonten, Prokaryonten, Eukaryonten), von denen das erstere das ursprünglichere ist, in dessen Verzweigungen sehr viele wärmeresistente („hyperthermophile“) Vertreter solcher „Archaebakterien“ angesiedelt sind. Man sollte damit zu Recht erwarten, dass hyperthermophile und chemoautotrophe Archaeoprokaryonten das Bild der ältesten bakteriellen Mikrofloren bestimmt haben. Im Gegensatz dazu hat die bisher bekannte paläontologische Überlieferung Befunde geliefert, die mehr auf Cyanobakterien (d. h. Prokaryonten) als auf Archaeoprokaryonten hinweisen. 
Wie dieses Beispiel zeigt, erfordern wichtige Einzelfragen der frühen organischen Evolution noch eine detaillierte Abklärung. Dazu gehört auch der mögliche Einfluss des kosmischen Bombardements vor etwa 3,8-4,0 Mrd. Jahren, das für die Kraterbildung auf der Mondoberfläche verantwortlich ist und das die frühe Erde in ähnlicher Weise betroffen haben musste (hier sind die Einschlagsstrukturen allerdings in der tektonisch aktiven Erdkruste später völlig zerstört worden). Obwohl dieses sog. „,späte“ Planetoiden-Bombardement sicher niemals zu einer flächendeckenden Sterilisation der Erdoberfläche geführt hat (vgl. Schidlowski, 1998), könnten diese Prozesse die frühe Evolution durchaus im Sinne einer positiven Selektion von robusten hyperthermophilen Archaeoprokaryonten beeinflusst haben. Da die ältesten bisher bekannt gewordenen Sedimente keine Hinweise auf einen derartigen massiven Asteroiden-Einfall geliefert haben, muss dieses Bombardement auf jeden Fall deutlich vor 3,8 Mrd. Jahren beendet gewesen sein.

Abschließend sei noch bemerkt, dass die ältesten Dokumente irdischen Lebens eine wichtige Leitfunktion bei der Suche nach fossilen Lebensresten im außerirdischen Bereich besitzen. Das gilt insbesondere für den Mars, der in seiner Frühgeschichte durch lebensfreundliche Umweltbedingungen (einschließlich einer Wasserhülle) charakterisiert war, sodass wir dort mit einiger Wahrscheinlichkeit eine ähnliche Evolution von Lebensprozessen erwarten könnten, wie sie für die archaische Erde nachgewiesen ist. Speziell das Auftreten biosedimentärer Strukturen von stromatolithischem Typ sowie merklicher Gehalte von organischem Kohlenstoff in den ältesten Marsgesteinen würde die Frage nach ehemaligem Leben auf unserem Nachbarplaneten eindeutig positiv beantworten.

\section{Literatur}

Appel, P. W. U., Fedo, C. M., Moorbath, S. \& Myers, J. S. (1998): Recognizable primary volcanic and sedimentary features in a low-strain domain of the highly deformed, oldest known ( 3.7-3.8 Gyr) greenstone belt, Isua, West Greenland, Terra Nova, 10, 57-62

Arneth, J. D., Schidlowski, M., Sarbas, B., Goerg, U. \& Amstutz, G. C. (1985): Graphite content and isotopic fractionation between calcite-graphite pairs in metasediments from the Mgama Hills, Southern Kenya, Geochim. Cosmochim. Acta, 49, 1553-1560

Awramik, S. M. (1992): The history and significance of stromatolites. In: Schidlowski, M., Golubic, S., Kimberley, M. M., McKirdy, D. M. \& Trudinger, P. A., Early Organic Evolution: Implications for Mineral and Energy Resources, Springer, Berlin, 435-449 
Bottinga, Y. (1969): Calculated fractionation factors for carbon and hydrogen isotope exchange in the system calcite - carbon dioxide - graphite - methane - hydrogen - water vapour, Geochim. Cosmochim. Acta, 33, 49-64

Brasier, M. D., Green, O. R., Jephcoat, A. P., Kleppe, A. K., Van Kranendonk, M. J., Lindsay, J. F., Steele, A. \& Grassineau, N. V. (2002): Questioning the evidence for Earth's oldest fossils, Nature, 416, 76-81

Bridgwater, D., Allaart, J. H., Schopf, J. W., Klein, C., Walter, M. R., Barghoorn, E. S., Strother, P., Knoll, A. H. \& Gorman, B. E. (1981): Microfossil-like objects from the Archaean of Greenland: A cautionary note, Nature, 289, 51-53

Brocks, J. J., Logan, G. A., Buick, R. \& Summons, R. E. (1999): Archean molecular fossils and the early rise of eukaryotes, Science, 285, 1033-1036

Buick, R. (1991): Microfossil recognition in Archean rocks: An appraisal of spheroids and filaments from a 3500 my old chert-barite unit at North Pole, Western Australia, Palaios, 5, 441-459

Durand, B. (1980): Kerogen - Insoluble Organic Matter from Sedimentary Rocks, Editions Technip, Paris, 519 S.

Eglinton, G. \& Calvin, M. (1967): Chemical fossils, Sci. Am., 216, 32-43

Eliade, M. (1964): Die Schöpfungsmythen, Benziger, Einsiedeln, 264 S.

Fedo, C. M. \& Whitehouse, M. J. (2002): Metasomatic origin of quartz-pyroxene rock, Akilia, Greenland, and implications for Earth's earliest life, Science, 296, 1448-1451

Greenberg, J. M. (1984): The structure and evolution of interstellar grains, Scient. Am., 250, 124-135

Grotzinger, J. P. \& Knoll, A. H. (1999): Stromatolites in Precambrian carbonates: Evolutionary mileposts or environmental dipsticks? Annu. Rev. Earth Planet. Sci., 27, 313-358

Hayes, J. M. (1994): Global methanotrophy at the Archean-Proterozoic transition. In: Bengtson, S., Early Life on Earth (Nobel Symposium 84), Columbia University Press, New York, 220-236

Hayes, J. M., Kaplan, I. R. \& Wedeking, K. W. (1983): Precambrian organic geochemistry: Preservation of the record. In: Schopf, J. W., Earth's Earliest Biosphere: Its Origin and Evolution, Princeton University Press, Princeton, N. J., 93-134

Hofmann, H. J. (2000): Archean stromatolites as microbial archives. In: Riding, R. E. \& Awramik, S. M., Microbial Sediments, Springer, Berlin, 315-327 
Irvine, W. M. \& Knacke, R. F. (1989): The chemistry of interstellar gas and grains. In: Atreya, S. K., Pollack, J. B. \& Matthews, M. S., Origin and Evolution of Planetary and Satellite Atmospheres, University of Arizona Press, Tucson, 3-34

Ivanova, L. V., Chapina, O. S. \& Meleshik, V. A. (1988): Nakhodka kokkoidnykh mikrofitofossilij v metamorfizovannykh kremnyakh Rannego Proterozoya SSSR (Funde von kokkoiden Mikrofossilien in metamorphen Kieselschiefern des frühen Proterozoikums der UdSSR), Dokl. Acad. Sci. UdSSR, 303, 210-211

Jeans, J. (1931): The Mysterious Universe, Cambridge University Press, Cambridge, $221 \mathrm{~S}$.

Kandler, O. (1993): The early diversification of life. In: Bengtson, S., Early Life on Earth (Nobel Symposium 84), Columbia University Press, New York, 152-160

Knoll, A. H. \& Barghoorn, E. S. (1977): Archean microfossils showing cell division from the Swaziland System of South Africa, Science, 198, 396-398

Kreulen, R. \& Van Beek, P. C. J. M. (1983): The calcite-graphite isotope thermometer; data on graphite bearing marbles from Naxos, Greece, Geochim. Cosmochim. Acta, 47, 1527-1530

Krumbein, W. E. \& Cohen, Y. (1977): Primary production, mat formation and lithification chances of oxygenic and facultative anoxygenic cyanophytes (cyanobacteria). In: Flügel, E., Fossil Algae, Springer, Berlin, 37-56

Miller, S. L. (1953): A production of amino acids under possible primitive Earth conditions, Science, 117, 528-529

Mojzsis, S. J., Arrhenius, G., McKeegan, K. D., Harrison, T. M., Nutman, A. P. \& Friend, R. L. (1996): Evidence for life on Earth before 3,800 million years ago, Nature, 384, 55-59

Moorbath, S., O’Nions, R. K. \& Pankhurst, R. J. (1973): Early Archaean age for the Isua iron-formation, West Greenland, Nature, 245, 138-139

Muir, M. D. \& Grant, P. R. (1976): Micropaleontological evidence from the Onverwacht Group, South Africa. In: Windley, B. F., The Early History of the Earth, Wiley, London, 595-604

Nisbet, E. G. \& Sleep, N. H. (2001): The habitat and nature of early life, Nature, 409, 1083-1091

O’Leary, M. H. (1981): Carbon isotope fractionation in plants., Phytochemistry, 20, 553-567

Oparin, A. I. (1924): Proiskhozhdenie Zhizni (Die Entstehung des Lebens), Moskovskii Rabochii, Moskau, 128 S 
Park, R. \& Epstein, S. (1960): Carbon isotope fractionation during photosynthesis, Geochim. Cosmochim. Acta, 21, 110-126

Pflug, H. D. (1967): Structured organic remains from the Fig Tree Series (Precambrian) of the Barberton Mountain Land (South Africa), Rev. Palaeobot. Palynol., 5, 9-29

Pflug, H. D. (1978): Yeast-like microfossils detected in the oldest sediments of the Earth, Naturwissenschaften, 65, 611-615

Pflug, H. D. (1987): Chemical fossils in early minerals, Topics in Current Chemistry, 139, 1-55

Ronov, A. B., Yaroshevsky, A. A. \& Migdisov, A. A. (1990): Khimicheskoe Stroyenie Zemnoi Kory i Khimicheski Balans Glavnykh Elementov (Chemischer Aufbau der Erdkruste und chemische Bilanz der Hauptelemente), Izdatel'stvo Nauka, Moskau, $181 \mathrm{~S}$.

Rosing, M. T., Rose, N. M., Bridgwater, D. \& Thomsen, H. S. (1996): Earliest part of Earth's stratigraphic record: A reappraisal of the $>3.7 \mathrm{Ga}$ Isua (Greenland) supracrustal sequence, Geology, 24, 43-46

Rozanov, A. J. (2002): Bakterial'naya Paleontologiya (Bakterielle Paläontologie), PIN Russian Academy of Sciences, Moskau, 188 S.

Schidlowski, M. (1982): Content and isotopic composition of reduced carbon in sediments. In: Holland, H. D. \& Schidlowski, M.: Mineral Deposits and the Evolution of the Biosphere, Springer, Berlin, 103-122

Schidlowski, M. (1988): A 3,800-million-year isotopic record of life from carbon in sedimentary rocks, Nature, 333, 313-318

Schidlowski, M. (1993): The initiation of biological processes on Earth: Summary of empirical evidence. In: Engel, M. H. \& Macko, S. A., Organic Geochemistry, Plenum Press, New York, 639-655

Schidlowski, M. (1998): Beginnings of terrestrial life: Problems of the early record and implications for extraterrestrial scenarios. In: Hoover, R. B., Instruments, Methods and Missions for Astrobiology, Proc. Int. Soc. Opt. Engin. (SPIE), Bellingham, WA, 3441, 149-157

Schidlowski, M. (2001): Carbon isotopes as biogeochemical recorders of life over 3.8 Ga of Earth history: Evolution of a concept, Precambrian Res., 106, $117-134$

Schidlowski, M. Appel, P. W. U., Eichmann, R. \& Junge, C. E. (1979): Carbon isotope geochemistry of the $3.7 \times 10^{9} \mathrm{yr}$ old Isua sediments, West Greenland: Implications for the Archaean carbon and oxygen cycles, Geochim. Cosmochim. Acta, 43, 189-199 
Schidlowski, M., Hayes, J. M. \& Kaplan, I. R. (1983): Isotopic inferences of ancient biochemistries: Carbon, sulfur, hydrogen and nitrogen. In: Schopf, J. W., Earth's Earliest Biosphere: Its Origin and Evolution, Princeton University Press, Princeton, N. J., 149-186

Schidlowski, M. \& Aharon, P. (1992): Carbon cycle and carbon isotope record: Geochemical impact of life over 3.8 Ga of Earth history. In: Schidlowski, M., Golubic, S., Kimberley, M. M., McKirdy, D. M. \& Trudinger, P. A., Early Organic Evolution: Implications for Mineral and Energy Resources, Springer, Berlin, 147-175

Schopf, J. W. (1993): Microfossils of the Early Archean Apex Chert: New evidence of the antiquity of life, Science, 260, 640-646

Schopf, J. W. (1999): Cradle of Life, Princeton University Press, Princeton, N. J., $347 \mathrm{~S}$.

Schopf, J. W. \& Walter, M. R. (1983): Archean microfossils: New evidence of ancient microbes. In: Schopf, J. W., Earth's Earliest Biosphere: Its Origin and Evolution, Princeton University Press, Princeton, N. J., 214-239

Schopf, J. W. \& Packer, B. M. (1987): Early Archaean (3.3-billion to 3.5-billionyear-old) microfossils from Warrawoona Group, Australia, Science, 237, 70-73

Schopf, J. W., Kudryavtsev, A. B., Agresti, D. G., Wdowiak, T. J. \& Czaja, A. D. (2002): Laser-Raman imagery of Earth's earliest fossils, Nature, 416, 73-76

Strauss, H. \& Moore, T. B. (1992): Abundances and isotopic compositions of carbon and sulfur species in whole rock and kerogen samples. In: Schopf, J. W. \& Klein, C., The Proterozoic Biosphere, Cambridge University Press, Cambridge, 709-798

Summons, R. E., Jahnke, L. L., Hope, J. M. \& Logan, G. A. (1999):

2-Methylhopanoids as biomarkers for cyanobacterial oxygenic photosynthesis, Nature, 400, 554-557

Treibs, A. (1934): Chlorophyll- und Häminderivate in bituminösen Gesteinen, Erdölen, Erdwachsen und Asphalten. Ein Beitrag zur Entstehung des Erdöls, Liebigs Ann. d. Chemie, 510, 42-62

Valley, J. W. \& O’Neill, J. R. (1981): ${ }^{13} \mathrm{C} /{ }^{12} \mathrm{C}$ exchange between calcite and graphite: A possible thermometer in Grenville marbles, Geochim. Cosmochim. Acta, 45, 411-419

Van Zuilen, M. A., Lepland, A. \& Arrhenius, G. (2002): Reassessing the evidence for the earliest traces of life, Nature, 418, 627-630 
Wada, H. \& Suzuki, K. (1983): Carbon isotopic thermometry calibrated by dolomite-calcite solvus temperatures, Geochim. Cosmochim. Acta, 47, 697-706

Walsh, M. M. (1992): Microfossils and possible microfossils from the Early Archean Onverwacht Group, Barberton Mountain Land, South Africa, Precambrian Res., 54, 271-293

Walter, M. R. (1983): Archean stromatolites: Evidence of the Earth's earliest benthos. In: Schopf, J. W., Earth's Earliest Biosphere: Its Origin and Evolution, Princeton University Press, Princeton, N. J., 187-213

Westall, F., De Wit, M., Dann, J., Van der Gaast, S., De Ronde, C. E. J. \& Gerneke, D. (2001): Early Archean fossil bacteria and biofilms in hydrothermally-influenced sediments from the Barberton greenstone belt, South Africa, Precambrian Res., 106, 93-116 


\title{
Die sedimentäre Haut unseres Planeten
}

\author{
Reinhard Gaupp
}

\section{Einleitung}

Sedimentgesteine sind nach ihrem Volumen nur mit ca. 5\% an den obersten $17 \mathrm{~km}$ der Lithosphäre unseres Planeten beteiligt, sie bedecken jedoch mehr als drei Viertel der Erdoberfläche und tragen den mit Abstand größten Teil der für die Menschheit wichtigen Ressourcen.

Die Intention dieses Beitrages ist es, die große Bedeutung sedimentärer Gesteine für die Zukunft der Menschheit hinsichtlich Ernährung und Rohstoffversorgung, aber auch mit Blick auf ökologische Gefährdungen durch anthropogene Belastungen darzustellen. Schließlich sind sedimentäre Abfolgen auch hervorragende Dokumente paläoklimatischer, paläoökologischer oder geodynamischer Zustände der zurückliegenden Erdgeschichte und erlauben uns damit das Studium dieser Paläo-Umweltbedingungen. Über den Blick in die erdgeschichtliche Vergangenheit lassen sich Erkenntnisse gewinnen, die einerseits zum Verständnis heutiger Aspekte unserer Umwelt wichtig sind, die aber auch die künftigen Auswirkungen menschlicher Aktivitäten auf den Lebensraum besser abschätzen lassen. Sedimentäre Abfolgen bieten die detailreichsten und zeitlich bestauflösenden Informationen zur Rekonstruktion der Geschichte unserer Erde. 


\section{Sedimente und ihre Verbreitung}

Um die Bedeutung der sedimentären Hülle unserer Erde ermessen zu können, muss der Blick zunächst auf Entstehung, Verteilung, Dynamik und die besonderen Eigenheiten der Sedimente gelenkt werden.

Sedimente bzw. Sedimentgesteine sind als Gemenge verschiedener Minerale und organischer Substanzen aufzufassen, die an der Erdoberfläche unter dem Einfluss von Wasser, Wind, Eis, Schwerkraft oder mit der Beteiligung von Organismen abgelagert worden sind. Beispiele sind Sand, Tone, Salze und Kalkschlämme.
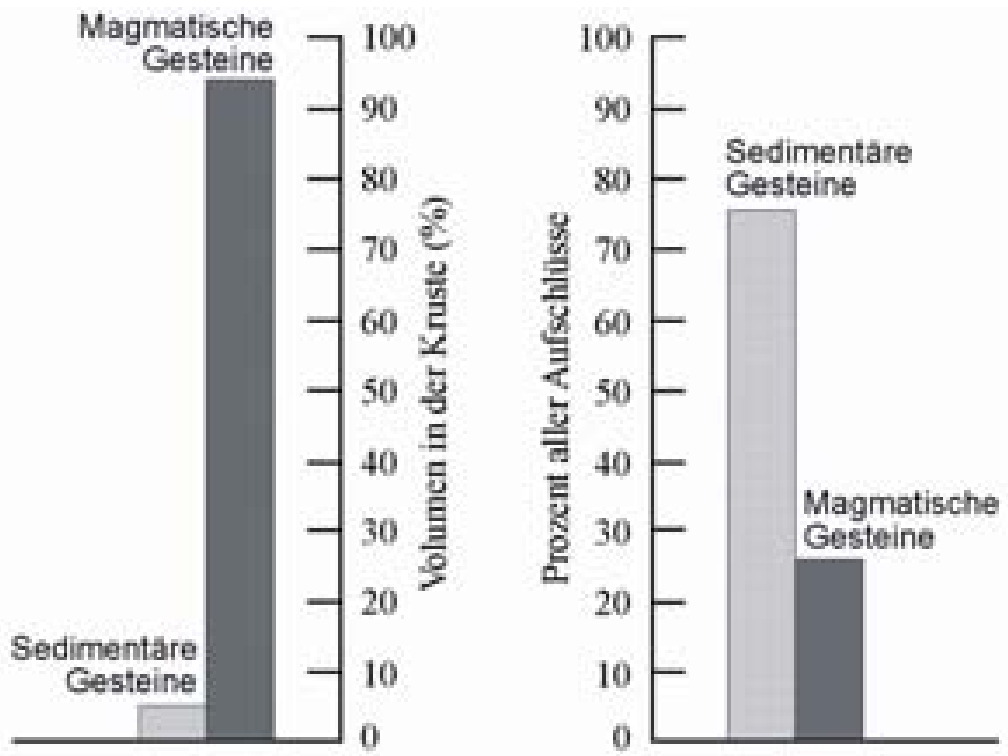

Abb. 1: Volumenanteil der Sedimente in der Erdkruste und an Aufschlüssen der Erdoberfläche (Skinner \& Porter 1987, Ibbeken \& Schleyer 1991).

Sedimente werden damit im obersten Teilraum der Geosphäre im Überlappungsbereich mit Atmosphäre, Hydrosphäre und Biosphäre gebildet. Sie entstehen gleichsam im direkten Erfahrungs- und Einflussbereich des Menschen. Im diesem „exogenen Teilkreislauf“ der Gesteine (Abb. 2) führen Verwitterung und Erosion von existierenden Gesteinen sowie Abtransport des partikulären und gelösten Materials zur Wiederablagerung von Sediment und schließlich zur Verfestigung neuer Sedimentgesteine. Bei der Versenkung in größere Erdtiefen von einigen 100 Metern bis mehreren Kilometern entstehen daraus Sedimentgesteine wie Sandstein, Tonstein oder Kalkstein. Erst in sehr großen Versenkungstiefen und/oder bei Temperaturen $>200{ }^{\circ} \mathrm{C}$ verlassen die Sedimentgesteine den „exogenen Teil“" des Gesteinskreislaufes (Abb. 2) und gehen in den „endogenen Teilkreislauf" mit Metamorphose, eventueller Aufschmelzung und Magmenbildung (Vulkanismus) über. 


\section{Der Gestejnsksejికlauf}

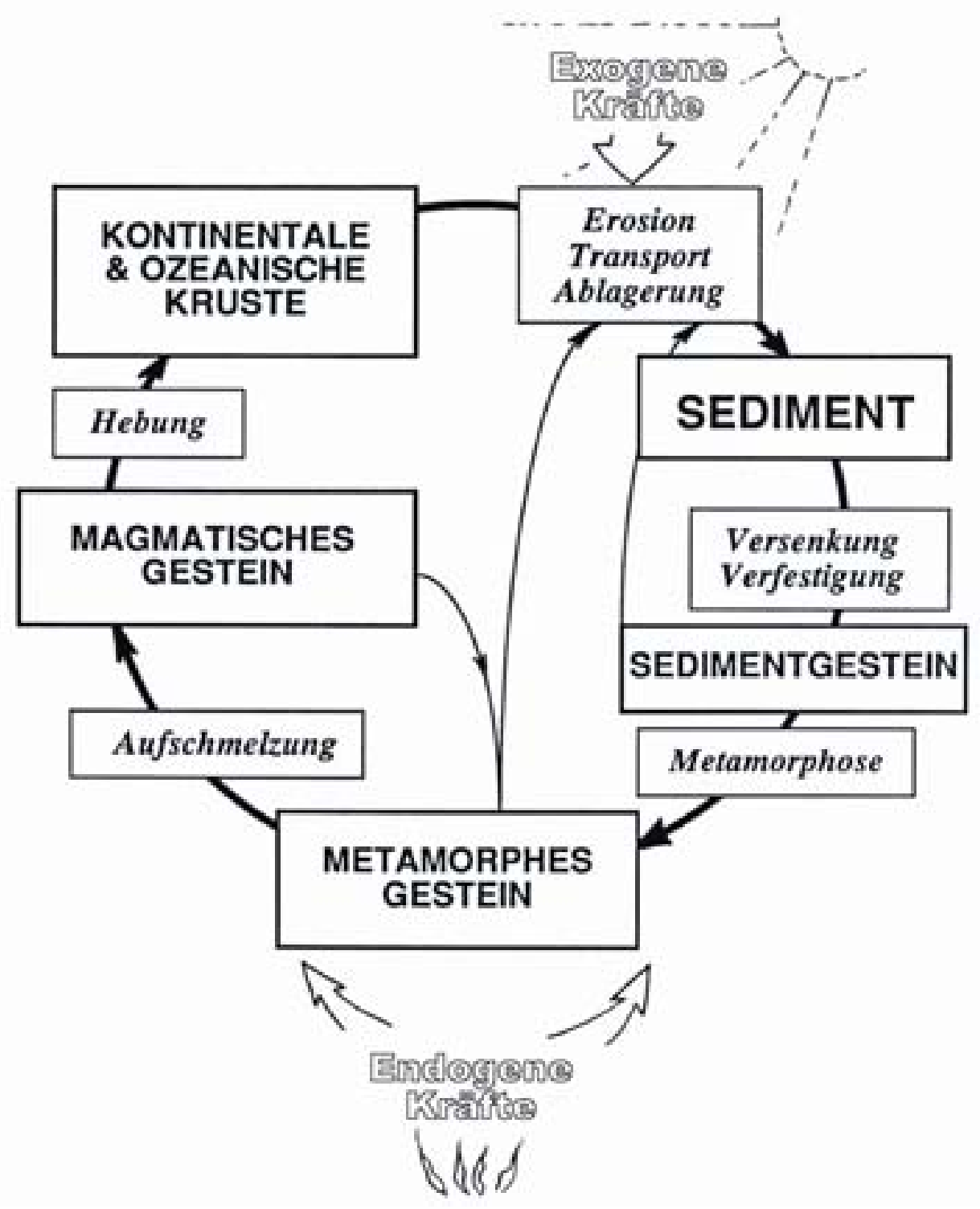

Abb. 2: Erosion, Abtragung, Transport, Ablagerung von Sediment, Versenkung bis in einen Temperaturbereich von ca. $200{ }^{\circ} \mathrm{C}$ und Verfestigung sind im Gesteinskreislauf als Exogener Teil definiert. Antriebskräfte des gesamten „Kreislaufs" bzw. dynamischen Systems sind Wärmeenergie aus dem Erdinneren und die Sonnenenergie. 
Jährlich werden ca. $8 \mathrm{~km}^{3}$ partikuläres Sedimentmaterial über die Flüsse ins Meer verfrachtet (Milliman \& Syvitski 1992, Leeder 1996). Dazu kommen noch ca. $2 \mathrm{~km}^{3}$ gelöste, mit dem Flusswasser dem Meer zugeführte Substanzen und eine Menge von ca. $0,91 \times 10^{9} \mathrm{t} /$ a feinstkörnigem Material, das über Windströmungen die Ozeane erreicht (Prospero 1996). Der Eintrag von kosmischem partikulärem Material aus dem interstellaren Raum in die Weltozeane ist demgegenüber heute nur verschwindend gering (3-4 x 10 t/a, Wassen et al. 1967, zit. in Degens 1989). Herkunftsgebiete der partikulären Fracht sind für den Windtransport vor allem die kontinentalen Trockengebiete der Erde. Für den Flusstransport stehen besonders große Mengen abgetragenen Materials in den vulkanreichen, reliefstarken, niederschlagsreichen tropischen Regionen Südostasiens zur Verfügung (Abb. 3). Zusammen mit den Flusssystemen, die den Himalaja und das Tibet-Plateau entwässern, wird in diesem Raum ca. $80 \%$ der globalen kontinentalen SedimentFracht den Meeren zugeleitet (Baumgartner \& Reichel 1975, Milliman \& Meade 1983, Degens 1989).

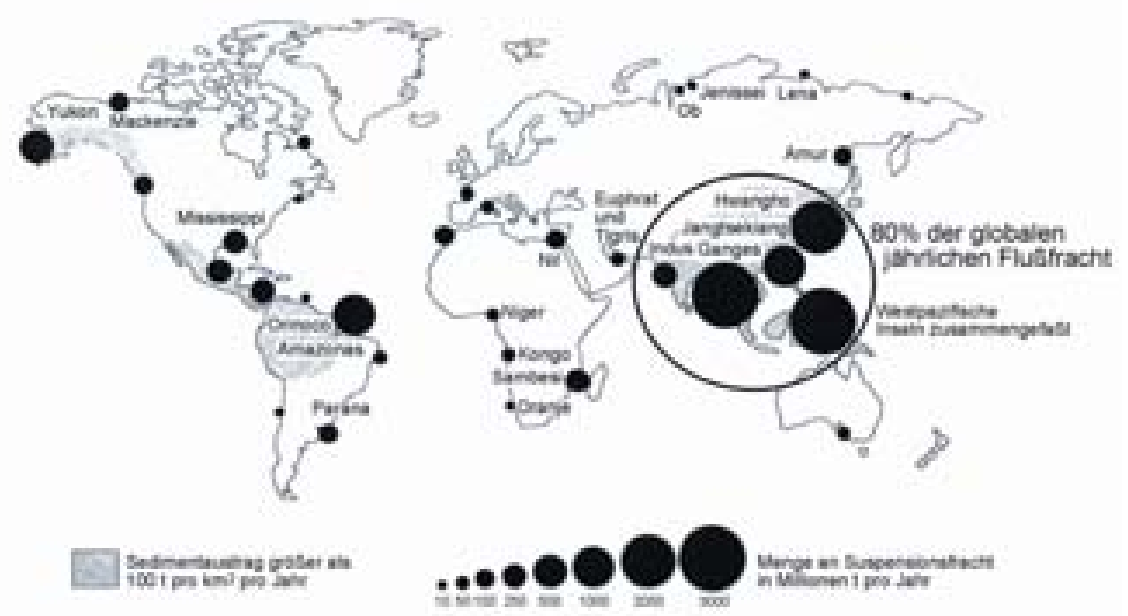

Abb. 3: Jährliche Flussfracht der großen Einqugsgebiete (Fütterer 2000).

Die Flüsse der Erde führen jährlich etwa $40000 \mathrm{~km}^{3}$ Wasser. Damit wird im Zeitraum von ca. 40000 Jahren einmal das Wasservolumen der Weltozeane über die kontinentalen verwitternden Oberflächen und Flusssysteme geleitet (Degens 1989).

Sedimentbecken sind nach Größe und Form vielfältige Senkungsräume bzw. Tiefzonen an der Erdoberfläche, die große Mengen an Sediment (bis mehr als 16 Kilometer mächtig) aufnehmen und über Zeiten von einigen Millionen bis zu einigen 100 Millionen Jahren (Veizer \& Jansen 1979, 1985) hinweg konservieren können, bis schließlich wieder Hebung durch erdinnere Kräfte zu Verwitterung und Erosion führen und einen neuerlichen Durchlauf eines exogenen Zyklus veranlassen. Ozeanbecken sind die ultimativen Zielräume des sedimentären 
Transportes. Jedoch nur ein relativ kleiner Teil des von den Kontinenten erodierten Materials erreicht die küstenferne Tiefsee, z. B. über Trübeströme oder Großrutschungen an den Kontinentalhängen. Der weitaus größte Anteil der kontinentalen Abtragungsmassen wird auf und an den Schelfgebieten der passiven Kontinentalränder abgelagert. An aktiven Kontinentalrändern, an denen Subduktion der ozeanischen Kruste stattfindet, wird die Ausbildung weiter stabiler Schelfflächen unterbunden. Dort wird das kontinentale Abtragungsmaterial in die Akkretionskeile über den Subduktionszonen integriert oder teilweise mit den abtauchenden ozeanischen Platten in den Mantel hinab subduziert. Die ozeanischen Becken enthalten nur Sediment mit sehr geringer Mächtigkeit.

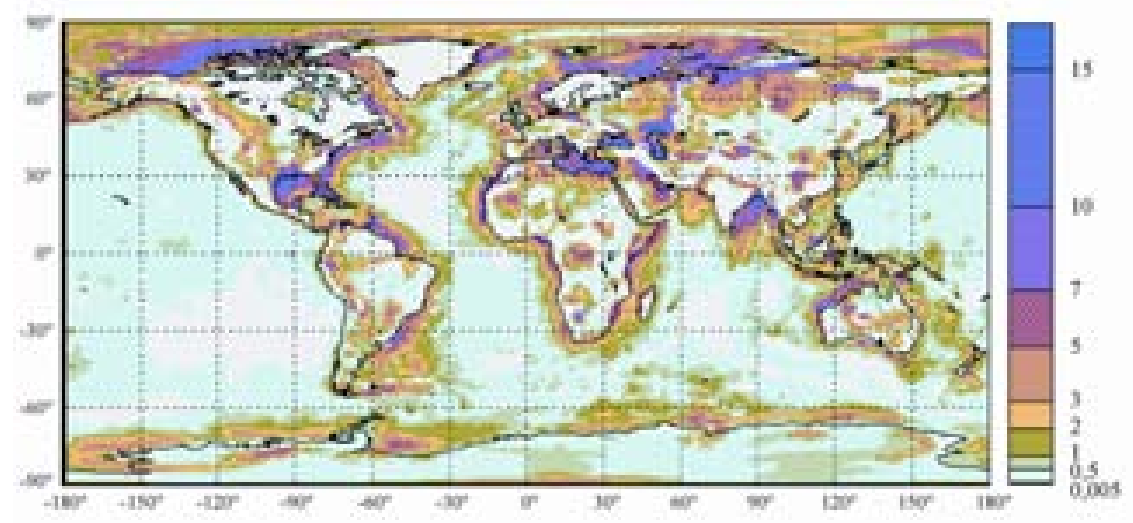

Abb. 4: Wie dick ist die sedimentäre Haut? Globale Verteilung der Sediment-Mächtigkeit. Skala in km, nach Laske \& Masters (1997).

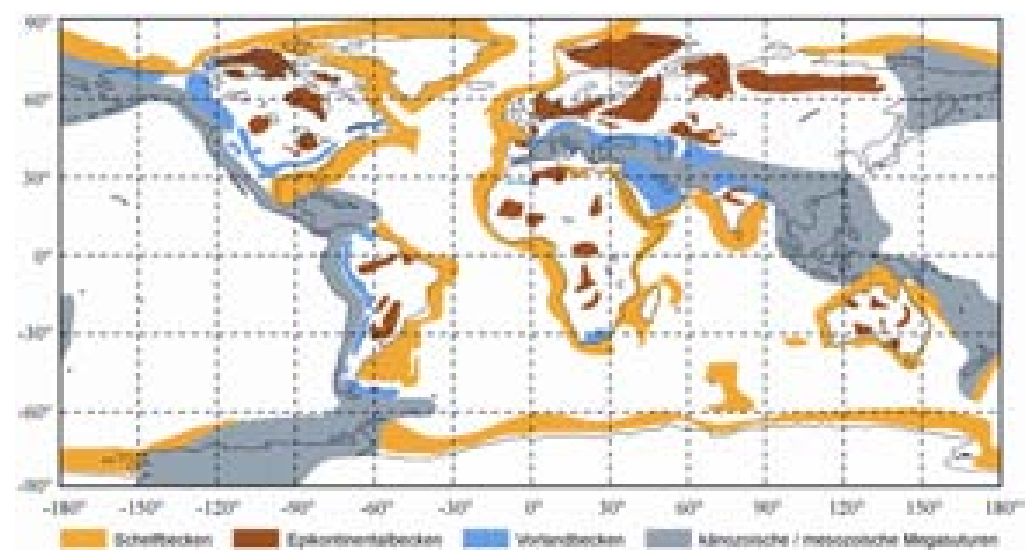

Abb. 5: Die wichtigsten Typen der Sedimentbecken, verändert nach Bally \& Snelson (1987).

Känozoische und mesozoische Megasuturen sind die alpidischen und zirkumpazifischen

Gebirgsgürtel, die in den let:ten ca. 200 Millionen Jahren gebildet worden sind und große

Sedimentmächtigkeiten einbezogen haben. 
Die flächen- und volumenmäßig größten Sedimentbecken sind damit die weiten Schelfflächen der passiven Kontinentränder mit gleichzeitig maximalen Mächtigkeiten der Sedimente bis $>12 \mathrm{~km}$ (Busby \& Ingersoll 1995). Diese asymmetrischen und bis über 200 Millionen Jahre überspannenden Sedimentansammlungen fangen den größten Teil der kontinentalen Sedimentanlieferung meeresrandnah ab. Ausnahmen von dieser Regel sind riesige submarine Fächersysteme, die sich vor Flussdeltas bilden, deren Flusseinzugsgebiete außergewöhnlich viel verfrachtbares Abtragungsmaterial bereitstellen (Allen 1997). Ein Beispiel ist der $>3500 \mathrm{~km}$ lange Bengal-Fächer, der sich an das Flusssystem von GangesBrahmaputra anschließt. Hier werden direkt kontinentale Abtragungsmassen aus dem Hochgebirge des Himalaja bis in die küstenferne Tiefsee verfrachtet (Einsele 2000). Weitere großvolumige Sedimentansammlungen bilden sich im Vorland aufsteigender Gebirge und nehmen direkt deren Abtragungsmassen auf (Vorlandbecken, Abb. 5; z. B. Molassebecken der Ostalpen oder der Anden). Kleinere Sedimentbecken mit geringeren Sediment-Mächtigkeiten sind kontinentale Gräben (z. B. Oberrhein, Ostafrika), Innerkontinental-Becken (z. B. Kongo-, TschadBecken), oder Becken in tektonisch aktivem, komplexem Rahmen (z. B. Tarim-, Quaidam-Becken nördlich von Tibet).

Schließlich gibt es intrakontinentale und langzeitig absinkende Becken oder Depressionen, deren Bildungsmechanismen trotz umfassender Datensätze bis heute kaum verstanden sind, wie z. B. die Zentraleuropäischen Becken zwischen England und Südpolen (van Wees et al. 2000).

Nur $0,5 \mathrm{~km}^{3}$ Sediment-Subduktion pro Jahr (Rückführung in den Erdmantel) stehen der jährlichen marinen Sediment-Produktion von 8 bzw. $10 \mathrm{~km}^{3}$ gegenüber. Damit sind die passiven Kontinentalränder (Schelfe) als Sammler der festländischen Abtragungsmassen die derzeitigen Anwachsgürtel der kontinentalen Kruste. Die Metamorphose der Sedimente und damit die Regeneration des kontinentalen Krustenvolumens findet an der Basis der mächtigen SchelfSedimentkeile ständig und besonders ausgeprägt in Zeiten von Kontinentkollisionen (Orogenesen, Gebirgsbildungen) statt. Rezyklierung von Sediment und metamorphem Sediment ist demnach heute der Normalfall. Die Wahrscheinlichkeit, mit der sich heute Sediment aus magmatischem oder metamorphem Gestein neu bildet, ist sehr gering ( $<10 \%$ nach Veizer \& Jansen 1979, 1985). Der größte Teil der heute gebildeten Sedimentmassen hat den exogenen Zyklus von Abtragung, Transport, Sedimentation und Gesteinsverfestigung bereits ein- oder mehrmals durchlaufen. 


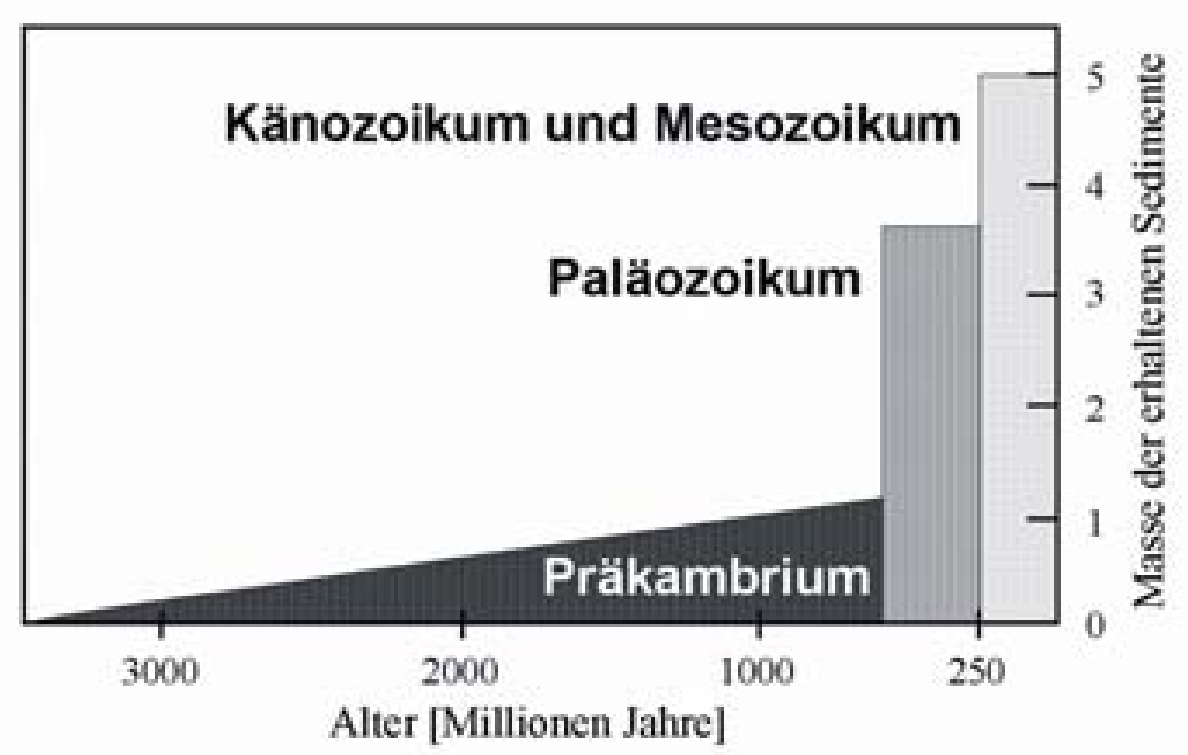

Abb. 6: Das Alter der erbaltenen Sedimentgesteine (Allegre 1994). Je älter die sedimentären Gesteine sind, desto weniger ist von ibnen erhalten. Aus Massenbilanzierungen und geochemischen Untersuchungen lässt sich ableiten, dass das gesamte Sedimentvolumen der Erde schon seit ca. 2,5 Milliarden Jabren in etwa gleich bleibt (Veizer \& Jansen 1979, 1985). SedimentgesteinsUmverteilung und-Reaykklierung bestimmen seither das Geschehen. Die Lebensdauer von Sedimentbecken ist aufgrund der Dynamik im exogenen Bereich meist < 500 Millionen Jahre.

\section{Die Bedeutung sedimentärer Gesteine für Wirtschaft und Gesellschaft}

Sedimente oder Sedimentgesteine sind das Substrat bester und guter landwirtschaftlich genutzter Böden in den kontinentalen Tiefländern, großen Flussniederungen und küstennahen Regionen. Sie sind deshalb in besonderer Weise eine Lebensgrundlage der Menschheit. Die Qualität der Böden ist zwar abhängig von Relief und Klima, das Substrat ist jedoch im wahren Sinne des Wortes von grundlegender Bedeutung. Besonders die jungen Lockersedimente, aber auch die meisten Sedimentgesteine lassen unter fast allen klimatischen Bedingungen die relativ besten Bodenentwicklungen zu. Sobald kristalline magmatische oder metamorphe Substrate auftreten, geht die Bodenqualität deutlich zurück. Eine positive Ausnahme bilden hier vor allem die gut verwitterbaren basaltischen Gesteine.

Gerade die dicht besiedelten küstennahen Tiefländer und Mündungsgebiete großer Ströme sind mit ihren jungen und tiefgründig verwitternden Lockersediment-Flächen die Gebiete intensivster Landwirtschaft. Für Mitteleuropa trifft dies bevorzugt für die guten Schwarzerden und Parabraunerden zu, die sich in den 
„Börden“ und Schwemmländern mit ihren Flusssedimenten und Lössauflagen bildeten.

Sedimente und Sedimentgesteine sind die mit Abstand wichtigsten GrundwasserTrägergesteine. Obgleich frei bewegliches Wasser auch in Tiefen bis ca. $10 \mathrm{~km} \mathrm{zu}$ finden ist, sind es lediglich die obersten wenigen hundert Meter unserer Geosphäre, die uns wirtschaftlich nutzbares Wasser bzw. Grundwasser bieten. Auch klüftiges nichtsedimentäres Gestein wie Granit oder Basalt kann hinreichend Grundwasser liefern. Der größte Teil der Wasserversorgung der Menschheit wird jedoch auch in Zukunft aus porösen Gesteinen bzw. Lockermassen gewonnen werden müssen, sofern er nicht direkt dem Oberflächenwasser von Flüssen und Seen entnommen wird (Baumgartner \& Reichel 1975). Auf die mit der Kontamination der Grundwasserträger verbundenen Probleme wird weiter unten eingegangen.

Sedimentgesteine sind Träger aller wichtigen Massenrohstoffe und aller fossiler Energierohstoffe. In Sedimentbecken werden für die Zukunft der Menschheit wesentliche Ressourcen gebildet und gespeichert (Wellmer \& Becker-Platen 2002). Dazu gehören in erster Linie die fossilen Energieträger, die zu $>80 \%$ die Weltenergieversorgung sicherstellen. Weiterhin wird der größte Teil des für die Trinkwasserversorgung wesentlichen Grundwassers aus Sedimentgesteinen gewonnen. Daneben sind Sedimentbecken auch Quellen für viele metallische und nicht-metallische Rohstoffe, Zuschlagstoffe, Baustoffe, Zementrohstoffe und Düngemittel. Sie werden als Standorte für Deponien hochtoxischer und radioaktiver Abfälle sowie als Speicher für Gase, Flüssigkeiten und Feststoffe benutzt. Schließlich kann aus porösen und klüftigen Medien der Sedimentbecken geothermische Energie gewonnen oder verflüssigtes $\mathrm{CO}_{2}$ entsorgt werden (KohlendioxidSequestrierung).

Angesichts einer auf die 10-Milliarden-Marke zustrebenden Weltbevölkerung und der raschen Entwicklung der Volkswirtschaften der bevölkerungsreichen Staaten Südostasiens wird sowohl der Energieversorgung als auch der Rohstoffsicherung sowie der Deponierung toxischer und radioaktiver Abfälle durch ein wissenschaftlich fundiertes Sedimentbecken-Management eine stark steigende Bedeutung zukommen.

Die Bedeutung der Ressourcen Koble, Erdöl und Erdgas ist evident, und es wird in Zukunft zur Deckung des Bedarfs an diesen Energieträgern notwendig sein, zur Aufsuchung und Produktion in immer größere Tiefen und in immer entlegenere Regionen der Erde vorzudringen, bis einmal der Verbrauch fossiler Energieträger durch Alternativen ersetzt werden kann. Um die Bohrkosten, die heute für tiefere Explorationsbohrungen meist zwischen 10 und 50 Millionen Dollar liegen, auf möglichst niedrigem Niveau zu halten, wird beispielsweise der Einsatz von aufwändigen geophysikalischen und geochemischen Methoden immer bedeutender. Immer größere Anforderungen werden an geowissenschaftliche ExplorationsModelle gestellt, um das Fehlschlagrisiko der Erdöl- und Erdgas-Bohrungen zu minimieren. 


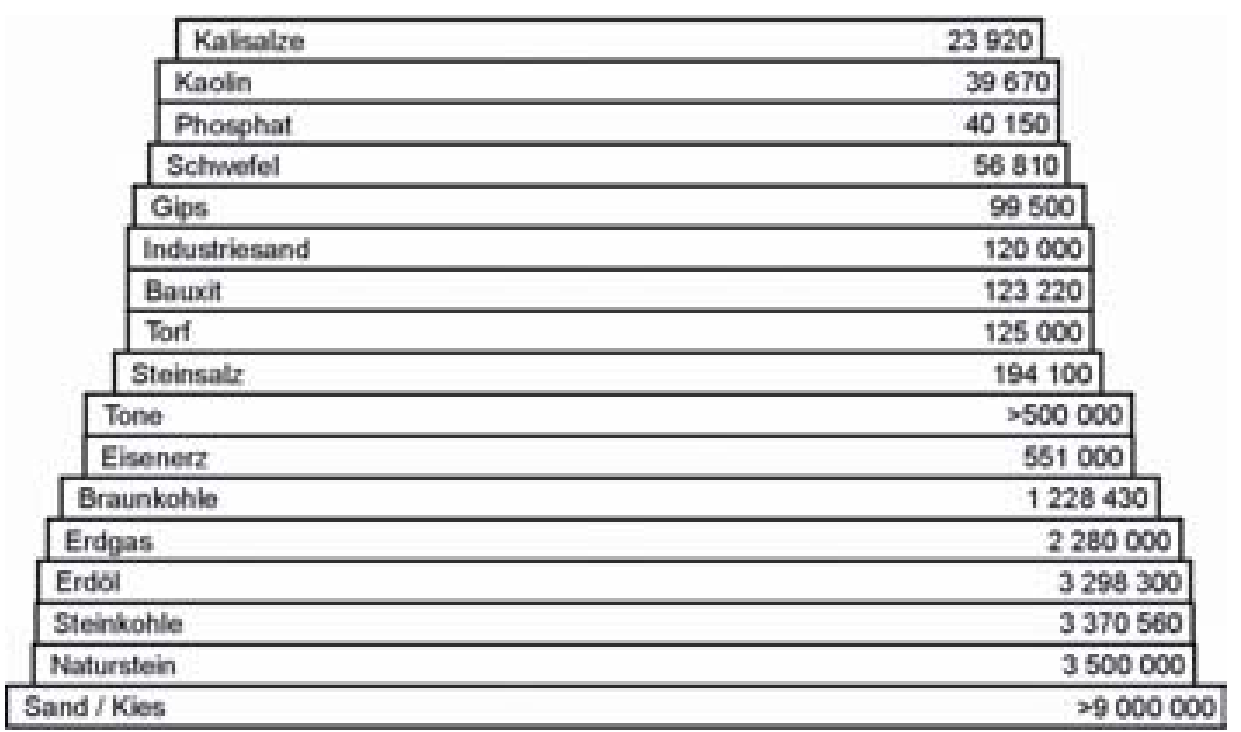

Abb. 7: Die 17 wichtigsten Massen- und Energie-Rohstoffe entstammen sedimentären Gesteinen oder sind sedimentärer Natur. Globale Produktion im Jahr 1996 nach der Menge (1000 t, Erdgas in Mio. m³, Wellmer \&o Becker-Platen 2002).

\begin{tabular}{|lr|lr|}
\hline Sand und Kies & $316 \mathrm{t}$ & Gips & $7,0 \mathrm{t}$ \\
Erdöl & $109 \mathrm{t}$ & Kaolin & $2,0 \mathrm{t}$ \\
Kalkstein, Dolomit & $94 \mathrm{t}$ & Aluminium & $1,5 \mathrm{t}$ \\
Steinkohle & $66 \mathrm{t}$ & Phosphat & $1,2 \mathrm{t}$ \\
Stahl & $33 \mathrm{t}$ & Kupfer & $1,0 \mathrm{t}$ \\
Tone & $22 \mathrm{t}$ & Kalium & $0,6 \mathrm{t}$ \\
Steinsalz & $11 \mathrm{t}$ & Schwefel & $0,5 \mathrm{t}$ \\
\hline
\end{tabular}

Tab. 1: Effektiver pro-Kopf-Verbrauch an mineralischen Rohstoffen (Massenrohstoffen) über ein 70-jähriges Leben in Deutschland (BGR 1998). 


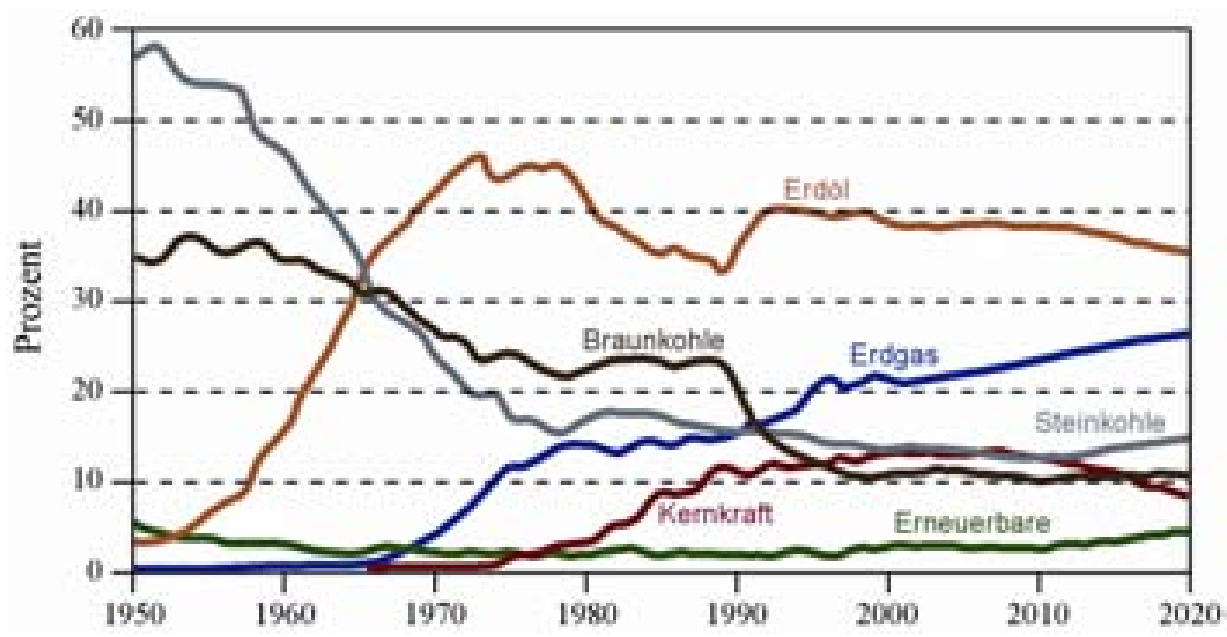

Abb. 8: Globaler Verbrauch und Zukunfts-Szenarien der wichtigsten Energiearten nach Rempel (2001). Den fossilen Energieträgern, die alle aus sedimentären Gesteinen gewonnen werden, kommt auch in den nächsten 50 Jahren größte Bedeutung zu.

\section{Sedimente: Vitale Bedeutung für die Menschheit}

Bis in Tiefen von mehr als $10 \mathrm{~km}$ sind Sedimentgesteine porös. Der vorhandene Porenraum ist meist mit Wasser gefüllt, das mehr oder weniger gelöste Substanzen enthält, auch Gase (wie Stickstoff oder Erdgas/Methan) oder organische Flüssigkeiten (Säuren, Öle, Kondensate). In größeren Erdtiefen und damit bei höheren Temperaturen und Drücken geht der für Sedimentgesteine so typische Porenraum fast gänzlich verloren. Das dort noch in geringsten Mengen vorhandene Wasser kann dann nicht mehr in Porensystemen mobil sein, sondern muss z. B. im Interkristallinraum der metamorphen Gesteine (Abb. 2) aktiv werden.

Hohe Anteile an Porenraum (bis $>40 \%$ des Gesteinsvolumens) sind also ein wichtiges Merkmal fast aller Sedimentgesteine in Erdoberflächen-Nähe. Dies erlaubt einen relativ raschen Transport von Flüssigkeiten und Gasen im Porenraum, unter anderem mit der Konsequenz der Akkumulation von Erdöl und Erdgas in Lagerstätten.

Die besondere Bedeutung von Sedimenten hängt auch mit ihrem hohen Anteil metastabiler Phasen zusammen, also mineralischen Bestandteilen, die unter den gegebenen Bedingungen der Oberflächennähe relativ rasch verändert oder gelöst werden können. Dies bewirkt ein hohes chemisches Reaktionspotential trotz der Niedrigtemperatur-Umgebung.

Sedimente und Sedimentgesteine bieten aufgrund ihrer Porensysteme und ihrer häufig sehr kleinen Mineralkomponenten mit großem Flächen-VolumenVerhältnis mitunter extrem hohe, ,innere“ und potentiell reaktive Oberflächen. Mehrere 100 Mio. km² Mineral-Oberflächen liegen im erdoberflächennahen Raum 
vor (Bosbach 1999). Auf diesen großen Flächen im Inneren der Sedimente finden ständig chemische und biochemische Prozesse statt, die in vielfacher Hinsicht für menschliches Leben von Bedeutung sind (z. B. Hochella 2002a).

Die Mobilität von Schwermetallen im Grundwasser und deren mögliche Fixierung durch Einbau bei Ausfällung von Mineralen aus den wässrigen Lösungen oder durch Sorption an Mineraloberflächen bzw. organische wie anorganische Kolloide wird in hohem Maße von der Verfügbarkeit dieser reaktiven Oberflächen gesteuert.

Bis zu ca. $120^{\circ} \mathrm{C}$ ist mit mikrobiellem Leben auf den inneren Oberflächen der Gesteine, vor allem der porösen Sedimentgesteine, zu rechnen. Diese Temperaturen werden normalerweise in 3 bis $4 \mathrm{~km}$ Tiefe erreicht. Noch sehr wenig wissen wir, in welchem Umfang und mit welcher Empfindlichkeit gegenüber Störungen das mikrobielle Leben unter der Erde einen reaktiven Puffer für menschliche Einwirkungen darstellt.

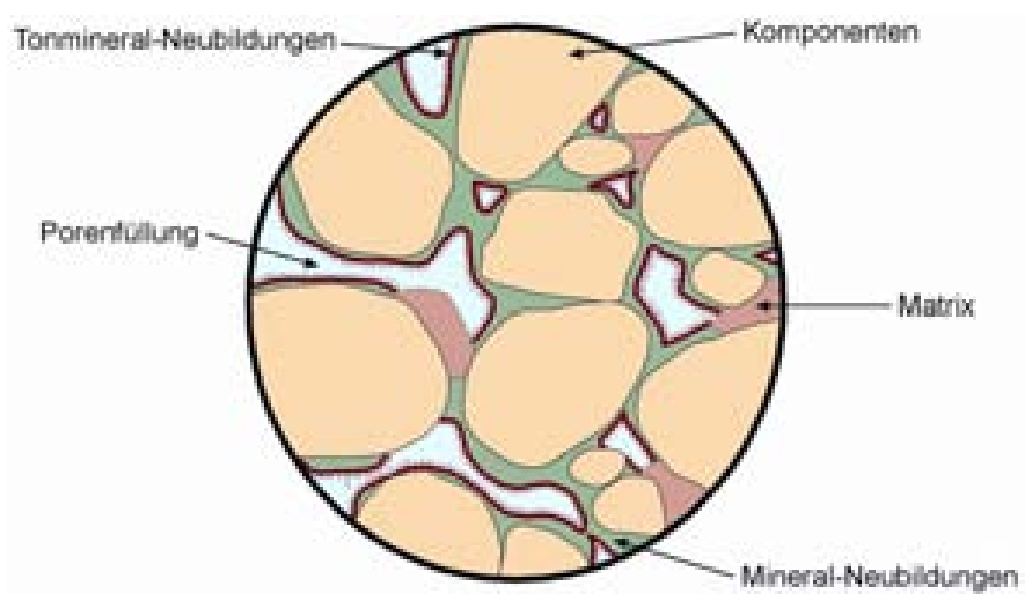

Abb. 9: Porensystem eines Sandstein-Grundwasserträgers. Besonders kritisch für die Austauschvorgänge zwischen Festkörper und Porenfüllung sind die Minerale (₹. B. Tonmineral-Neubildungen), Kolloide und organischen Substanzen (einschließlich der Mikroorganismen) auf den vergleichsweise leicht zugänglichen Poren-Grenaflächen (oder inneren Oberflächen) der porösen Medien.

Grenzflächenreaktionen zwischen Mineraloberflächen und der Füllung der Porenräume (Wasser mit gelöstem Material, Gase, organische Substanzen, aber auch Mikroorganismen) bestimmen die Lebensbedingungen in unserer Umwelt und beeinflussen den Austausch von klimarelevanten Treibhausgasen mit der Atmosphäre.

Sedimente sind als Quelle und Senke von Schadstoffen anzusehen. Kristallisations- und Auflösungsprozesse spielen dabei eine große Rolle. Die Auflösung von Sulfidmineralen unter oxischen Bedingungen birgt erhebliche Umwelt- 
risiken, so etwa auf Bergbauhalden mit sulfidischem Material (Acid Mine Drainage Problem).

Für geotechnische Anwendungen werden deshalb schon heute oberflächenspezifische Reaktionen eingesetzt, um auf Morphologie (Kristallform), Keimbildung bzw. Kristallisations-Kinetik Einfluss zu nehmen. Oberflächen-sensitive und lokal wie energetisch hochauflösende Methoden (Claret et al. 2002) werden zunehmend in diesen Zweigen der angewandten Umweltforschung eingesetzt, unter anderem auch um die Belastung von Böden besser einschätzen und ihr natürliches Potential der Selbstreinigung von den Prozessen her gründlicher verstehen zu lernen (Gerth \& Förstner 1991). Organische und metallische Schadstoffe, persistierende organische Verbindungen, Pestizide und Herbizide etc. reichern sich durch das Rückhaltevermögen in Böden und sedimentären Grundwasserträgern z. T. in gefährlicher Weise an (z. B. Grathwohl 1994, Hochella 2002a). Das Management dieser langzeitigen Anreicherungen von Stoffen z. B. in Wasser-Trägergesteinen (Untergrundkontaminationen) mit ihrer potentiellen Wirkung auf die Grundwasserqualität ist zu einer zwingenden Zukunftsaufgabe geworden.

Die sedimentären Gesteine Ton und Salz sind als geologische Barrieren für Endlagerstandorte (Gömmel 1997, AkEnd 2002) von großer Bedeutung. Zur Gewährleistung der Isolation radioaktiver Abfälle darf nur eine geringe Grundwasserbewegung im Gesteinskörper des „einschlusswirksamen Gebirgsbereichs“ vorhanden sein. Außerdem muss die flächenhafte Ausdehnung und die vertikale Mächtigkeit $(\geq 100 \mathrm{~m})$ des Wirtsgesteins für kritisches Material ausreichend groß sein für die Realisierung eines sicheren Endlagers. In Salz und Tongesteinen werden hierfür sehr viel kleinere Flächen benötigt, als z. B. für Granit. Tonsteine haben gegenüber Salzgesteinen und Granit den weiteren Vorteil, dass sie mit ihrem hohen Tonmineralanteil und dessen Sorptionseigenschaften ein hohes Rückhaltevermögen gegenüber Schadstoffen (z. B. Schwermetalle, organische Verbindungen, Radionuklide) besitzen. Die feinklastischen Sedimentgesteine, die Tone, sind auch bei der Behandlung von Altlasten, der oberflächennahen Untergrund-Kontamination und in der Deponietechnik im Einsatz.

\section{Sedimentgesteine als Archive für Paläozustände der Erde}

Atmosphäre, Klima, Hydrologie, Organismen, fluviale oder ozeanische Strömungen und viele weitere Einflussgrößen an der Oberfläche unseres Globus kontrollieren die Merkmalsausbildung sedimentärer Gesteine. Damit werden diese Gesteine seit den frühesten Phasen der Erdgeschichte (umfassender überliefert seit ca. 3,5 Mrd. Jahren) zu wichtigen Archiven der ehemaligen Bedingungen auf der Oberfläche unseres Erd-Systems.

So lässt sich die frühe Zusammensetzung und Entwicklung der frühen Atmosphäre unseres Planeten anhand spezifischer (meta-)sedimentärer Gesteine teil- 
weise rekonstruieren. Minerale wie Pyrit $\left(\mathrm{FeS}_{2}\right)$ oder Uraninit $\left(\mathrm{UO}_{2}\right)$ können nicht unter sauerstoffreichen Oberflächenbedingungen existiert haben, denn dort wären sie oxidiert worden. Sind sie aber Bestandteil der Komponenten von z. B. konglomeratischen Gesteinen, dann kann daraus auf Sauerstoff-Armut der Paläoatmosphäre vor 4-2,5 Mrd. Jahren geschlossen werden (Schidlowski 1988, siehe auch Beitrag Schidlowski in diesem Band).

Die Entwicklungsgeschichte der Sedimente selbst und deren sich im Laufe der Erdgeschichte ändernde Zusammensetzung erlaubt Aussagen zu den Veränderungen der Zusammensetzung des Meerwassers auf der frühen Erde (Kempe \& Degens 1985), zur Verwitterungsintensität unter einer frühen $\mathrm{CO}_{2}$-reichen Atmosphäre und zur Zunahme der Rezyklierung von Sediment (Veizer \& Jansen 1979, 1985).

Glaziale Ablagerungen, von Gletschern in Äquatornähe und auf Niveau des Meeresspiegels gebildet, sind Zeugen für außergewöhnliche Vereisungszustände der Erde vor ca. 700 Mio. Jahren (Hofmann \& Schrag 2002). Die „SnowballEarth"-Hypothese geht davon aus, dass damals nahezu die gesamte Erdoberfläche für lange Zeiträume von einer mächtigen Eisdecke überzogen war.

Die heute mit großem Aufwand betriebene und international koordinierte (paläo-)ozeanographische Forschung greift in erheblichem Maße auf die Sedimente der Meeresböden zurück, um beispielsweise die Ausbreitung von Meereis, die Veränderungen von Oberflächen-/Bodentemperaturen und Meeresströmungen, die Schwankungen des Windeintrages von partikulärem Material, die Ursachen von Planktonblüten oder die Raum-Zeit-Verteilung von marinen AussterbeEreignissen zu rekonstruieren (Wefer et al. 1999).

Maar-Seen stellen ausgezeichnete Paläoklima-Dokumente dar, die aufgrund der fast ungestörten Abfolge jahreszeitlicher Feinschwankungen im Sediment höchste Auflösung und damit zeitlich sehr präzise Rekonstruktion von Umweltbedingungen zumindest der jüngsten Erdgeschichte erlauben (Zolitschka et al. 2000). In koordinierten internationalen Großforschungsvorhaben (z. B. PAGES) werden See-Sedimente verteilt über alle Kontinente in mehreren Transekten global untersucht mit dem Ziel, die Klimageschichte über den Zeitraum der letzten 300000 Jahre $\mathrm{zu}$ studieren. Besonderes Interesse finden erdgeschichtliche Zeiträume, während denen außerordentlich rasche Veränderungen der Klimaparameter (Temperatur, Niederschlagsintensität, Windaktivität etc.) stattfanden. Sofern die oft vielfältigen Ursachen solcher Schwankungen rekonstruiert werden können, wird eine Einschätzung der Störanfälligkeit des heutigen globalen Klima und eventuell auch eine Prognose künftiger klimatischer Entwicklung möglich. Der Blick in die erdgeschichtliche Vergangenheit mithilfe sedimentärer Dokumente lässt uns damit auch erkennen, wie rasch natürliche, vom Menschen nicht beeinflusste Klimaänderungen abgelaufen sind und welche ökologischen Wirkungen damit verknüpft waren.

Aber nicht nur die Paläoklima-Rekonstruktionen sind aktuelle und reizvolle Forschungsziele, Sedimente bergen darüber hinaus einen fast unermesslichen Schatz an Informationen über die Entwicklung des Lebens auf der Erde. Die 
Fossilinhalte sedimentärer Gesteine sind die Grundlage der Paläontologie und Evolutionsbiologie. Phylogenetische Abstammungs-Beziehungen müssen aus den sicherlich sehr unvollständig und lückenhaft konservierten Lebensresten abgeleitet werden. Aber auch der Ablauf und die Ursachen der großen biotischen Krisen der Erdgeschichte, während derer sich die Biodiversität drastisch reduzierte, lassen sich nur aus Fossildokumenten und Sedimenteigenschaften erfassen (z. B. Prothero 1990, Oschmann et al. 2000).

Der oberflächennahe Ausdruck der geodynamischen Entwicklung der Erde zeigt sich z. B. in der sich ändernden Zusammensetzung von sedimentären Gesteinen, die während der Auffaltung eines Gebirges gebildet werden (von Eynatten \& Gaupp 1999, Schlunegger 1999, Kuhlemann et al. 2001). Hebung, Exhumation (Tiefenfreilegung) und Erosion eines Gebirges führen zum Eintrag von zunehmend tiefergebildetem Gesteinsmaterial in die Sedimentationsräume oder lassen Einzugsgebiete von material-exportierenden Flusssystemen im Laufe der Gebirgsbildung größer werden.

\section{Aspekte aktueller Forschung}

Aus der Vielfalt aktueller innovativer Entwicklungsrichtungen der Sediment-Forschung seien nur einige Beispiele herausgegriffen. Zunächst einerseits im Großmaßstab der Sedimentbecken die dreidimensionale Erfassung von Raumstrukturen, andrerseits der nanoskalige Weg in immer höher auflösende Analysen von z. B. Mineraloberflächen.

Die Fortschritte in der Bearbeitung von 3D-seismischen Untergrunddaten erlauben immer präziser zielgerichtete Explorationsbohrungen z. B. auf Erdgas oder Erdöl. Nicht nur die räumlichen Details werden sichtbar bis in den Maßstab von Zehnermetern, sondern auch Speichereigenschaften lassen sich bis zu einem gewissen Maß besser einschätzen, als das mit zweidimensionaler Seismik möglich war. In der künftigen weiteren Verbesserung sowohl der technischen Verfahren, als auch der sedimentgeologischen Modelle und Konzepte lassen sich die Explorations-Risiken minimieren.

Am anderen Ende der Betrachtungs-Maßstäbe stehen die „Nanogeowissenschaften“. Das Verständnis der grundlegenden Prozesse auf Mineraloberflächen im atomaren Maßstab ist vor allem im exogenen Bereich der Sedimente nötig, um Einsicht in globale Wechselwirkungen innerhalb geochemischer Stoffkreisläufe zu ermöglichen. Nur durch das direkte Beobachten der Reaktionsmechanismen im atomaren Maßstab und deren Modellierung können viele globale Prozesse im Hinblick auf ein Erdmanagement verstanden und vorausgesagt werden (z. B. Astilleros et al. 2001, Bosbach 1999, Hochella 2002b). Auch die biogeochemischen Wechselwirkungen zwischen Mineral-Oberflächen und Mikroorganismen in den Sedimenten werden in der Zukunft einmal auf molekularer Ebene definierbar. 


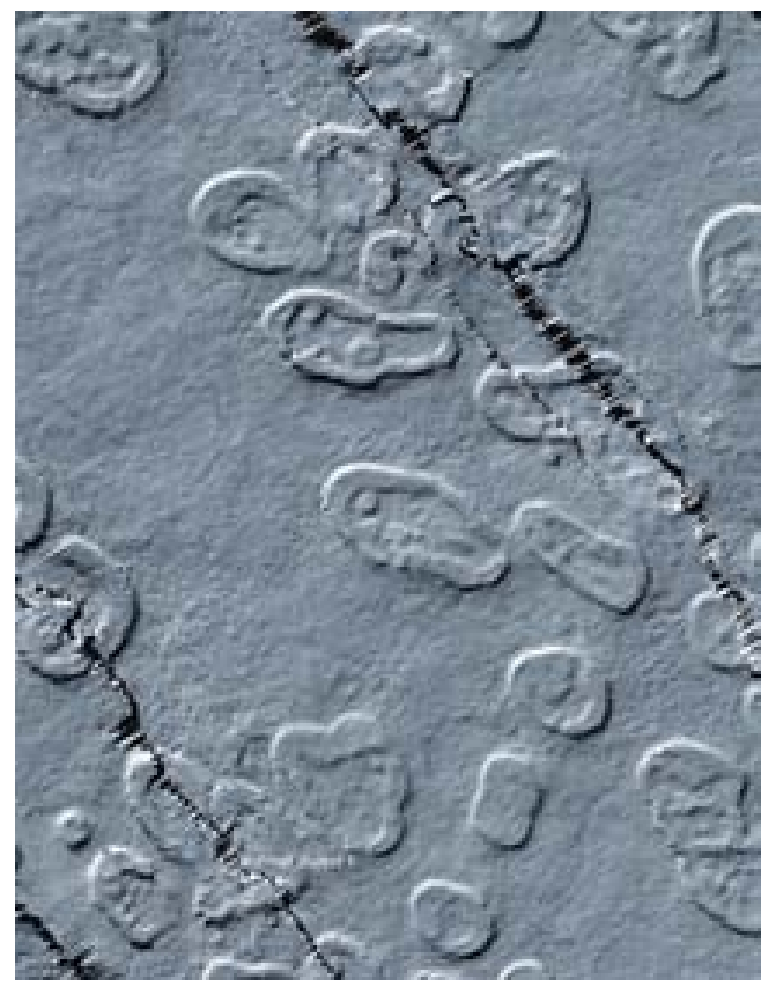

Abb. 10: Räumliche Darstellung (schattierte Aufsicht) von miozänen Atoll-Riffen vor Tunesien, die beute in einer Tiefe von ca. $700 \mathrm{~m}$ unter dem Meeresboden liegen (Bildbreite ca. $10 \mathrm{~km}$ ). Durch das Processing dreidimensionaler seismischer Daten lassen sich die geologischen Strukturen und Raumdetails sowie gesteinsphysikalischen Eigenschaften dieser potentiellen Speichergesteine erfassen (Kiefer \& Stabl 2002).

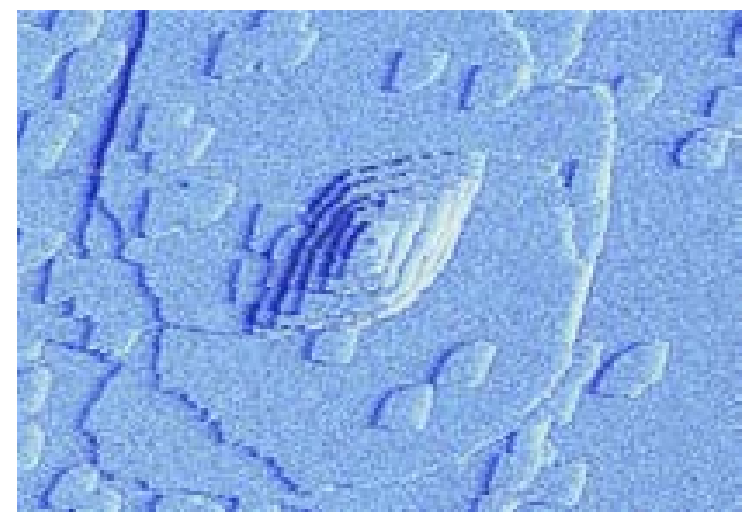

Abb. 11: Darstellung der molekularen Strukturen auf der Oberfläche eines Bariumsulfat-Kristalls mit der Rasterkeraftmikeroskopie (AFM,) nach Bosbach in Harjes \& Emmermann 1999;

Stufenhöhe ca. 0,35 $\mathrm{nm}$. 
Multidisziplinäre Ansätze fachgrenzenüberschreitender und zukunftsweisender Forschung sind zu erkennen, z. B. auch in der Analyse von geobiologischen Prozessen bei der Bildung und Umgestaltung von Sedimentgesteinen, in der Untersuchung von Raum-Zeit-Variationen von Fluidbewegungen im sedimentären Untergrund und bei der dynamischen Modellierung von Sediment-Wasser-Wechselwirkungen. Künftige Untersuchungen der sedimentären Haut unserer Erde werden sich methodischer Ansätze mit größerer Bandbreite der Skalen und noch höherer Auflösung bedienen können und die Integration großer Datenvolumen aus sehr unterschiedlicher Herkunft anstreben, um ein tieferes Verständnis unserer Lebensumwelt zu erreichen.

\section{Zusammenfassung und Ausblick}

1. Sedimente sind Substrat bester und guter landwirtschaftlich genutzter Böden in den kontinentalen Tiefländern, großen Flussniederungen und küstennahen Regionen. Sie sind damit eine Lebensgrundlage der Menschheit.

2. Sedimentgesteine sind die Träger aller wichtigen Massenrohstoffe und aller fossiler Energierohstoffe und die wichtigsten Grundwasser-Trägergesteine.

3. Sedimentgesteine sind mit ihrem umfassenden Porensystem und ihrer extremen inneren Oberfläche wichtige reaktive Puffer für anthropogene Verunreinigungen.

4. Sedimentgesteine sind Archive der Erdgeschichte (Paläoklima, Paläohydrologie, Palökologie, Evolutionsbiologie, Geodynamik etc.) mit dem Potential sehr hoher zeitlicher Auflösung. Sedimente und Sedimentgesteine zu studieren verbessert das Verständnis heutiger Prozesse und ermöglicht Prognosen für die nahe Zukunft (Variabilität und künftige Veränderbarkeit).

5. Das geowissenschaftliche Studium der Sedimente kann ein Zeitverständnis vermitteln, eine Einsicht in die Geschwindigkeiten natürlicher abiotischer Abläufe und in deren Empfindlichkeit für Störungen. Klimazukunft hat hier zwar bereits einen hohen Stellenwert in der öffentlichen Beachtung, stellt aber nur einen von weiteren möglichen geowissenschaftlichen Beiträgen zur Prognose von Veränderungs-Geschwindigkeiten dar (siehe auch Küsten-Dynamik, Fluss-Management, desaster prevention in steep slope management usw.). Nicht das technische Beherrschen, sondern Kenntnisse natürlicher Veränderungsraten können hier vonseiten der Geowissenschaften beigetragen werden.

Die sedimentäre Haut unserer Erde als Interaktionszone der globalen Sphären ist verletzlich und bedarf der vorsorgenden und pfleglichen Nutzung. 


\section{Literatur}

AkEnd (2003): Auswahlverfahren für Endlagerstandorte.- Empfehlung des AkEnd - Arbeitskreis Auswahlverfahren Endlagerstandorte, $45 \mathrm{~S}$.

Allègre, C. (1994): From Stone to Star - A View of Modern Geology, Harvard Univ. Press, Cambridge, $287 \mathrm{~S}$.

Allen, P. A. (1997): Earth Surface Processes, Blackwell Science, Oxford, 404 S.

Astilleros J. M., Pina C. M. Férnandez-Diaz L. and Putnis A. (2002): Molecular scale surface processes during the growth of calcite in the presence of manganese, Geochimica Cosmochimica Acta 66, 3177-3189

Bally, A. W. \& Snelson, S. (1987): Realms of Subsidence. In: S. Foster, N. H. \& Beaumont, E. A. (eds.), Geologic Basins I - Classification, Modeling, and Predictive Stratigraphy, Treatise of Petroleum Geology Reprint Series No.1, Amer. Assoc. Petrol. Geol., Tulsa, 1-86

Baumgartner, A.. \& Reichel, E. (1975): The world Water Balance, Oldenburg Verlag, München - Wien, 179 S.

Bosbach, D. (1999): Mineraloberflächen - von atomaren Prozessen zur

Geotechnik. In: Harjes, H.-P. \& Emmermann, R. (1999): Geotechnologien: Das „System Erde“ - Vom Prozessverständnis zum Erdmanagement, Senatskommission für Geowissenschaftliche Gemeinschaftsforschung der DFG, 99-107

Busby, C. J. \& Ingersoll, R. V. (1995): Tectonics of Sedimentary Basins, Blackwell Scientific, 579 S.

Claret, F., Bauer, A., Schäfer, T., Griffault, L., \& Lanson, B. (2002): Experimental investigations of the Interaction of Clays with High pH Solutions: A Case Study from the Callovo-Oxfordian Formation, Meuse - Haute Marne Underground Laboratory (France), Clays and Clay Minerals, 50(5), 633-646

Degens, E. T. (1989): Perspectives on Biogeochemistry, Springer Verlag, Berlin, $423 \mathrm{~S}$.

Einsele, G. (2000): Sedimentary Basins, Evolution, Facies, and Sediment Budget, 2. Ed., Springer Verlag, $792 \mathrm{~S}$.

Eynatten, H. von, \& Gaupp, R. (1999): Provenance of Cretaceous synorogenic sandstones in the Eastern Alps: constraints from framework petrography, heavy mineral analysis, and mineral chemistry, Sed. Geol. 124, 81-111

Füchtbauer, H. (1988): Sedimente und Sedimentgesteine - Teil II der Serie Sediment-Petrologie, Schweizerbart, Stuttgart, 1141 S. 
Fütterer, D. K. (2000): The Solid Phase of Marine Sediments. In: Schulz, H. D. \& Zabel, M. (Eds.), Marine Geochemistry, Springer Verlag Berlin, 1-28

Gerth, J. \& Förstner, U.(1991): Die Belastung von Böden. In: Warnecke, G., Huch, M. \& Germann, K. (eds.), Tatort Erde, menschliche Eingriffe in Naturraum und Klima, Springer Verlag Berlin, 51-72

Gömmel, R. (1997): Endlagerung radioaktiver Abfälle, Spektrum der Wissenschaft 97(1), 98-105

Grathwohl, P. (1994): Persistenz organischer Schadstoffe in Boden und Grundwasser - können einmal entstandene Untergrundverunreinigungen wieder beseitigt werden? In: Matschullat, J. \& Müller, G. (eds.): Geowissenschaften und Umwelt, Springer Verlag Berlin, 263-273

Harjes, H.-P. \& Emmermann, R. (1999): Geotechnologien: Das „System Erde“ Vom Prozessverständnis zum Erdmanagement, Senatskommission für Geowissenschaftliche Gemeinschaftsforschung der DFG, 140 S.

Hillier, S. (1995): Erosion, sedimentation, and sedimentary origin of clays. In: Velde, B. (ed.) Origin and mineralogy of clays, Springer Verlag Berlin, 162-219

Hochella, M. F. (2002a): Sustaining Earth: Thoughts on the present and future roles of mineralogy in environmental science, Mineralogical Magazine, 66/5, 627-652

Hochella, M. F. (2002b): Nanoscience and technology - the next revolution in the Earth sciences, Earth and Planetary Science Letters, 203, 593-605

Hofmann, P. F. \& Schrag, D. P (2002): The snowball Earth hypothesis: testing the limits of global change, Terra Nova 14, 129-155

Ibbeken, H. and Schleyer, R. (1991): Source and sediment, Springer-Verlag, Berlin - Heidelberg, $286 \mathrm{~S}$.

Janson-Smith, D. \& Cressey, G. (eds.) (1996): Earth's Restless Surface, The Natural History Museum London, 60 S.

Kempe, S. \& Degens (1985): An early Soda Ocean? Chem. Geol. 53: 95-108

Kiefer, E. \& Stahl, E. (2002): Visualization of the growth history of an Upper Miocene reef tract offshore Tunisia by advanced 3D-coherence processing, Zbl. Geol. Paläont. Teil I, 2001, 3/4, 211-236

Kuhlemann, J., Frisch, W., Dunkl, I., Székely, B. (2001): Quantifying tectonic versus erosive denudation: The Miocene core complexes of the Alps, Tectonophysics, 330: 1-23

Laske, G. \& Masters, G. (1997): A Global Digital Map of Sediment Thickness, EOS Trans. AGU, 78, F483 
Leeder, M. (1996): Sedimentary Basins: tectonic recorders of sediment discharge from drainage catchments, Earth Surface Processes and Landforms, 22, 229237

Leeder, M. (1999): Sedimentology and Sedimentary Basins - from Turbulence to Tectonics, Blackwell, Oxford, 592 S.

Milliman, J. D. \& Meade, R. H. (1983): World-wide delivery of river sediment to the oceans, J. Geol. 91, 1-21

Milliman, J. D. \& Syvitski, J. P. M. (1993): Geomorphic/tectonic control of sediment discharge to the ocean: The importance of small mountainous rivers, J. Geol. 100, 525-544

National Research Council (1997): The Dynamics of Sedimentary Basins - Panel on the Geodynamics of Sedimentary Basins, U. S. Board on Earth Sciences and Resources, Washington, D. C., 43p

Oschmann, W., Dullo, Ch., Mosbrugger, V. \& Steiniger, F. F. (2000): Evolution des Systems Erde - Geobiologische und paläobiologische Prozesse als Antrieb.- Kl. Senckenberg-Reihe Nr. 35, 57 S.

PAGES - Past Global Changes; www.pages-igbp.org

Prospero, J. M. (1996): The atmospheric transport of particles in the ocean. In: Ittekot, V., Schäfer, P., Honjo, S. \& Depetris, P. J. (eds.), Particle Flux in the Ocean. Wiley \& Sons, Chichester, 19-52

Prothero, D. R. (1990): Interpreting the Stratigraphic record, W. H Freeman \& Co., New York, $410 \mathrm{~S}$.

Rempel, H. (2001): Erdgas im 21. Jahrhundert, Vortrag Frühjahrstagung DGMK Celle, BGR, www.bgr.de/b123/erdgas_21.pdf

Schidlowski, M. (1988): Die Erdatmosphäre im Präkambrium - Entwicklung des atmosphärischen Sauerstoffs, Die Geowissenschaften, 7, 212-217

Schlunegger, F. (1999): Controls of surface erosion on the evolution of the Alps: constraints from the stratigraphies of the adjacent foreland basins, Int. J. Earth Sci. 88, 285-304

Siever, R. (1988): Sand - Ein Archiv der Erdgeschichte, Spektrum Verlag, Heidelberg, $254 \mathrm{~S}$.

Skinner, B. J. \& Porter, S. C. (1987): Physical Geology, John Wiley \& Sons, New York, $750 \mathrm{~S}$.

Van Wees, J.-D., Stephenson, R. A., Ziegler, P. A., Bayer, U., McCann, T., Gaupp, R., Narkiewicz, M., Nielsen, S. \& Scheck, M. (2000): On the origin of the Southern Permian Basin, Central Europe, Marine and Petroleum Geology, Volume 17/1, 43-59 
Veizer, J. \& Jansen, S. L. (1979): Basement and sedimentary recycling and continental evolution, J. Geol., 87, 341-370

Veizer, J. \& Jansen, S. L. (1985): Basement and sedimentary recycling 2: time dimension to global tectonics, J. Geol., 625-643

Wefer, G., Berger, W. H., Bijma, J, \& Fischer, G. (1999): Clues to Ocean History: A Brief Overview of Proxies. In: Fischer, G. \& Wefer, G. (eds.), Use of Proxies in Paleoceanography, Springer, 1-68

Wellmer, F.-W. \& Becker-Platen, J. D. (2002): Sustainable development and the exploitation of mineral and energy resources: a review, Int. J. Earth Sci., 91, 723-745

Wilgus, C. K. et al. (1988): Sea-Level Changes: An Integrated Approach, Soc. Econ. Paleont. Mineral. (SEPM) Special Publ. No. 42, Tulsa, 407 S.

Windley, B. F. (1995): The Evolving Continents, John Wiley, Chichester, 52

Zolitschka, B., Brauer, A., Stockhausen H., Lang, A. \& Negendank, J. F. W. (2000): Annually dated Late Weichselian continental paleoclimate record from the Eifel, Germany, Geology, 28, 783-786 


\title{
Wenn Kristalle fließen und Schmelzen hüpfen
}

\author{
Sharon L. Webb
}

\section{Einleitung}

Was bewegt die Erde? Die einzelnen Schritte der Atome im Innern unseres Planeten bewegen die Erdplatten und verursachen gewaltige vulkanische Ausbrüche. Die Bewegungsfähigkeit von Atomen in Schmelzen und Kristallen verursacht die zauberhaften physikalischen Eigenschaften von irdischer Materie. Jede atomare Bindung in einem Kristall oder einer Schmelze hat eine bestimmte Lebenszeit - abhängig von der Struktur des Materials, dem Druck und der Temperatur. Wenn sich das Material, in einer Zeit kürzer als die längste Bindungslebenszeit, verformen soll, so zeigt es elastische Eigenschaften. Diese Eigenschaften sind es, die das Material für kurze Zeit spröde und zerbrechlich machen, sodass es hüpfen und springen kann. Wenn das Material sich verformen soll, in einer Zeit, die länger als die längste Bindungslebenszeit ist, so zeigt das Material viskose Eigenschaften. Dies bedeutet, dass das Material - über einen langen

Zeitraum betrachtet - über Tausende von Kilometern fließen kann. Das Fließen von Kristallen und Hüpfen von Schmelzen belebt unseren Planeten und macht ihn zu einem noch bezaubernderen Ort, als wir dachten. 


\section{Unser Planet}

Unser Planet ist ein schöner und bequemer Ort zum Leben. Er schwebt blauschimmernd im schwarzen All und ist still und friedlich. Aber dieser Schein trügt! Unser Heimatplanet ist alles andere als ruhig. Unser Planet ist dynamisch: immer im Umbruch begriffen und ständig in Bewegung. Nichts bleibt wie es ist, und nichts ist von Dauer. Wir wissen, dass sich die Erdplatten - von den Geowissenschaftlern auch tektonische Platten genannt - ständig bewegen. So entfernt sich New York beispielsweise jedes Jahr um $\sim 20 \mathrm{~cm}$ weiter von Frankfurt am Main, und der Indische Kontinent rast mit derselben Geschwindigkeit nach Norden und baut dabei den Himalaja immer wieder neu auf. In 50 Millionen Jahren wird das Gesicht von Mutter Erde ganz anders ausschauen als heute. Das Mittelmeer wird nicht mehr existieren. Das bedeutet: kein schöner Sommerurlaub mehr in Griechenland oder auf den Deutschen liebster Ferieninsel Mallorca. Stattdessen können sie die nun subtropisch gewordene Antarktis besuchen!

Die tektonisch bedingten Bewegungen an der Erdoberfläche sind das Endergebnis des Brodelns im Innern der Erde. Unsere lebhafte Erdkugel baut sich aus einem inneren, festen und einem äußeren, flüssigen Kern auf. Für den Menschen ist jedoch der hierauf folgende kristalline Erdmantel von größerer Bedeutung: Es ist die ungestüme Dynamik dieses Mantels, die die Platten bewegt und sich somit für die großen Veränderungen an der Oberfläche verantwortlich zeigt. Der Mantel ist es, der wieder neue Kruste aufbaut und er ist es auch, der die alte Kruste frisst.

An den mittelozeanischen Rücken - den Nahtstellen der Erdschollen - ist die Kruste sehr dünn, und das heiße Mantelmaterial kommt näher an die Oberfläche als irgendwo sonst. Wegen des niedrigeren Drucks beginnt der Mantel partiell aufzuschmelzen. Die Schmelze sammelt sich unter dem ozeanischen Rücken, und wegen des Dichteunterschieds zwischen heißer Schmelze und kaltem Umgebungsgestein bahnt sich das flüssige Gestein einen Weg nach oben, wo es neuen Ozeanboden bildet. Auf der anderen Seite beginnt hingegen die Subduktion - d. h., dass alte, erkaltete Kruste wieder in den Mantel abtaucht. Während also an den ozeanischen Rücken durch fortwährende Krusten-Neubildung die Platten weiter auseinander gepresst werden, wird die andere Seite der Platte schon wieder in den Mantel hineingezogen. Auch auf diesen Prozess wirkt sich der Konvektionsstrom des Mantels noch unterstützend aus.

Es gibt noch andere Mechanismen, durch die neues Material an die Erdoberfläche gelangt: Einer davon hat seine Ursache in den Mantelplumes. Die Plumes sind heißes, kristallines Material, dessen Geburtsstätte an der Kern-Mantel-Grenze in $2900 \mathrm{~km}$ Tiefe zu finden ist. Wegen des Dichteunterschieds zwischen dem heißen Gestein und dem kühlerem Mantel-Gestein steigt der Plume bis auf eine Tiefe von $200 \mathrm{~km}$ auf. Dort wird die Hitze des Materials gebraucht, um Krustenund Mantelmaterial aufzuschmelzen. Diese Schmelze gelangt dann bei weiterem Aufstieg durch Vulkanausbrüche an die Oberfläche. Es gibt auch vulkanische Ausbrüche hinter subduzierten Platten. Das wasserhaltige Sediment an der Ober- 
fläche der untertauchenden Platte hat einen niedrigen Schmelzpunkt und beginnt bereits in einer Tiefe von $100 \mathrm{~km}$ zu schmelzen. Das Wasser von diesen Sedimenten gelangt auch in den kristallinen Mantel oberhalb der absinkenden Platte. Da die Anwesenheit von Wasser den Schmelzpunkt von Gesteinen erniedrigt, fängt dieser Teil des oberen Mantels auch an, teilweise zu schmelzen. Die in ihrer Zusammensetzung unterschiedlichen Schmelzen wandern wegen des Dichteunterschieds nach oben, bilden Magmakammern, die ausbrechen und dabei Vulkane formen.

Außer den Bewegungen der tektonischen Platten und den Vulkanausbrüchen gibt es auch noch weitere Anzeichen für die dynamischen Aktivitäten des Erdinnern. Nach einem Erdbeben kann man sehen, wie weit und in welche Richtung sich der Boden bewegt hat. Zum Beispiel kann man an Bahngleisen nach einem Beben große Falten beobachten, nachdem der Erdboden um $2 \mathrm{~m}$ in eine Richtung verschoben wurde. In Regionen, in denen es ständig zu Mini-Erdbeben kommt, kann man den Effekt der 1-cm-Bewegungen im Jahr anhand von regelmäßig gepflanzten Reihen in Baum-Plantagen ausmachen: Auf der einen Seite der Störung bewegen sich die Bäume nicht, sind also ortsfest. Auf der anderen Seite bewegen sich die übrigen Reihen um wenige Zentimeter im Jahr auseinander - ohne großen Aufruhr! Die Bäume auf dem sich bewegenden Land halten Abstand zueinander, und nach einigen Jahren bekommt man einen schönen Knick in den ansonsten linear verlaufenden Baumreihen, welche die Störung durchkreuzt.

Die Vulkane an der Oberfläche sind auch sehr gute Wegweiser für die Drift der Erdschollen. In dem Gebiet, in dem eine Platte subduziert wird, gibt es Vulkane, die von der teilweise geschmolzenen, untertauchenden Platte mit Magmen gefüttert werden. Noch eindrucksvoller sind die Ketten von Vulkanen, wie sie von Mantelplumes verursacht werden. Die Stelle des Plumes in der Tiefe ist fest verankert, und die Stelle des resultierenden Vulkans darüber bleibt auch geometrisch fest auf der Platte verankert. Die Platte jedoch bewegt sich weiter und fährt über den heißen Fleck (Hot Spot) im Erdmantel hinweg. Hierbei wird eine Kette von Vulkanen erzeugt. Wenn der Kontakt des Vulkans zum Plume abbricht, muß er zwangsläufig verhungern - er erlischt! Das Magma drängt jedoch weiter immer wieder von unten nach und bahnt sich schließlich einen neuen Weg nach oben, die Folge: Ein neuer Vulkan entsteht! Die Hawaii-Emperor-Sea-Mount-Kette, die von Hawaii bis Kamtschatka reicht, ist so ein Resultat eines fest verankerten Plumes und einer sich bewegenden Platte. Vor der Hauptinsel Hawaii gibt es bereits einen neuen submarinen Vulkan, Loihi genannt. Dieser jüngste Sprössling des hawaiianischen Hot-Spots wird sich allerdings erst in 50000 Jahren über den Meeresspiegel erheben. Doch danach ist noch lange nicht Schluss, denn dieser Prozess wird sich noch sehr lange fortsetzen und immer neue tropische Urlaubsinseln bilden. Diese könnten dann das verschwindende Mittelmeer als zukünftige Urlaubs-Paradiese ersetzen. 
Es gibt noch andere wunderbare Ereignisse an der Oberfläche, die von den unruhigen Vorgängen unter unseren Füßen zeugen. Da gibt es zum Beispiel die faszinierenden, effusiven Lavaströme, wie es sie auf Hawaii oft zu sehen gibt. Hier, auf einer verwüsteten Lava-Welt, brechen aus der nur äußerlich erkalteten, schwarzgewordenen Lavazunge immer wieder neue, intensiv rote und gelbe Flüsse hervor. Man kann dabei oft hinreißende Formen in der mal schneller und mal langsamer fließenden Lava erkennen. Es gibt auch die spektakuläreren Vulkane, wie den Ätna auf Sizilien, bei dessen Ausbrüchen glühendes Magma in die Luft geschleudert wird. Hier ist die ganze Pracht und der Aufruhr der Erde zu sehen.

Die Aufgabe der Geowissenschaftler ist es, unseren Planeten besser zu verstehen. Was der Planet ist und wie die einzelnen Teile zu einem Ganzen zusammenpassen, um schließlich das Gesamtbild in seiner ganzen natürlichen Schönheit erfassen zu können. Um so eine große Unternehmung vollbringen zu können, brauchen wir Laboruntersuchungen und physikalische Modelle von unserem Planeten. Wir müssen herausfinden, welchen Chemismus die Erde hat, welcher Druck und welche Temperaturen in großen Tiefen herrschen und welche Minerale unter diesen Bedingungen noch stabil sind.

Zur Überprüfung der Realitätsnähe unserer Modelle vergleichen wir die im Labor gemessenen physikalischen Eigenschaften mit denen, die von der Erde selbst gemessen worden sind. Die physikalischen Eigenschaften, die von der Erde selbst gemessen wurden, sind das Trägheitsmoment - von dem wir die Dichte ableiten können; die magnetischen und elektrischen Felder, die uns vor hochenergetischen, kosmischen Partikeln schützen und uns zeigen, in welcher Richtung Norden liegt und - nicht zuletzt - die Geschwindigkeit der seismischen Wellen durch die Erde. Diese Wellengeschwindigkeiten sind die auskunftsreichsten Daten.

Von den seismischen Geschwindigkeitsmessungen wissen wir, dass die Erde einen Schalenaufbau besitzt und dass es mit steigendem Druck und Temperatur Phasenumwandlungen von Mineralen gibt. Ebenfalls bekannt ist, wie hoch die Geschwindigkeiten der Wellen durch diese Minerale in der Erde sind. Das bedeutet, dass wir lediglich unter denselben Druck- und Temperaturbedingungen wie sie in der Erde vorherrschen, Geschwindigkeitsmessungen an den möglichen Mineralen durchführen müssen, um diese dann mit den uns zur Verfügung stehenden Daten der Erde vergleichen zu können. Damit sollte sich eigentlich herausfinden lassen, ob das Mineral in der Erde existieren kann. Zusammen mit der Wellengeschwindigkeit haben wir jetzt auch die Dämpfung der seismischen Welle, wenn sie die Erde durchdringt. Das gibt uns Auskunft über kinetische Prozesse innerhalb der Erde, die Energie verbrauchen, um sich zu vollenden.

Die Ergebnisse der Arbeit der Seismologen sind, dass wir ein Bild vom Erdinnern in Form von Wellengeschwindigkeiten und Dämpfung haben. Diese Daten zeigen erhöhte Geschwindigkeit und niedrige Dämpfung für alte, kalte Lithosphäre, wenn sie in hunderte von Kilometern in die Erde hinein abtaucht. Wir haben auch Gebiete mit hoher Dämpfung und niedriger Geschwindigkeit für 
heiße und vielleicht schmelzehaltige Mantelplumes, wenn Material aus der Tiefe nahe an die Oberfläche dringt.

Um physikalische Eigenschaften, wie Wellengeschwindigkeit und Dämpfung, im Labor messen zu können, müssen wir zunächst über die Messmethoden Bescheid wissen. Wir müssen Spannung, Verformung und Verformungsraten messen können, lernen, sie richtig zu deuten und die Laborergebnisse und -simulationen immer wieder neu mit denen der Erde vergleichen und unsere Experimente den Vorgaben der Natur anpassen.

Die Erde gibt aber ihre Geheimnisse nicht so ohne weiteres preis, zumindest nicht, ohne dass man hart dafür arbeiten und um die richtige Positionierung jedes einzelnen, kleinen Puzzleteils kämpfen müsste. Ein solches Beispiel, wie irreführend die Planeten sein können, kommt von einer Untersuchung der Mondgesteine aus dem Jahre 1970.

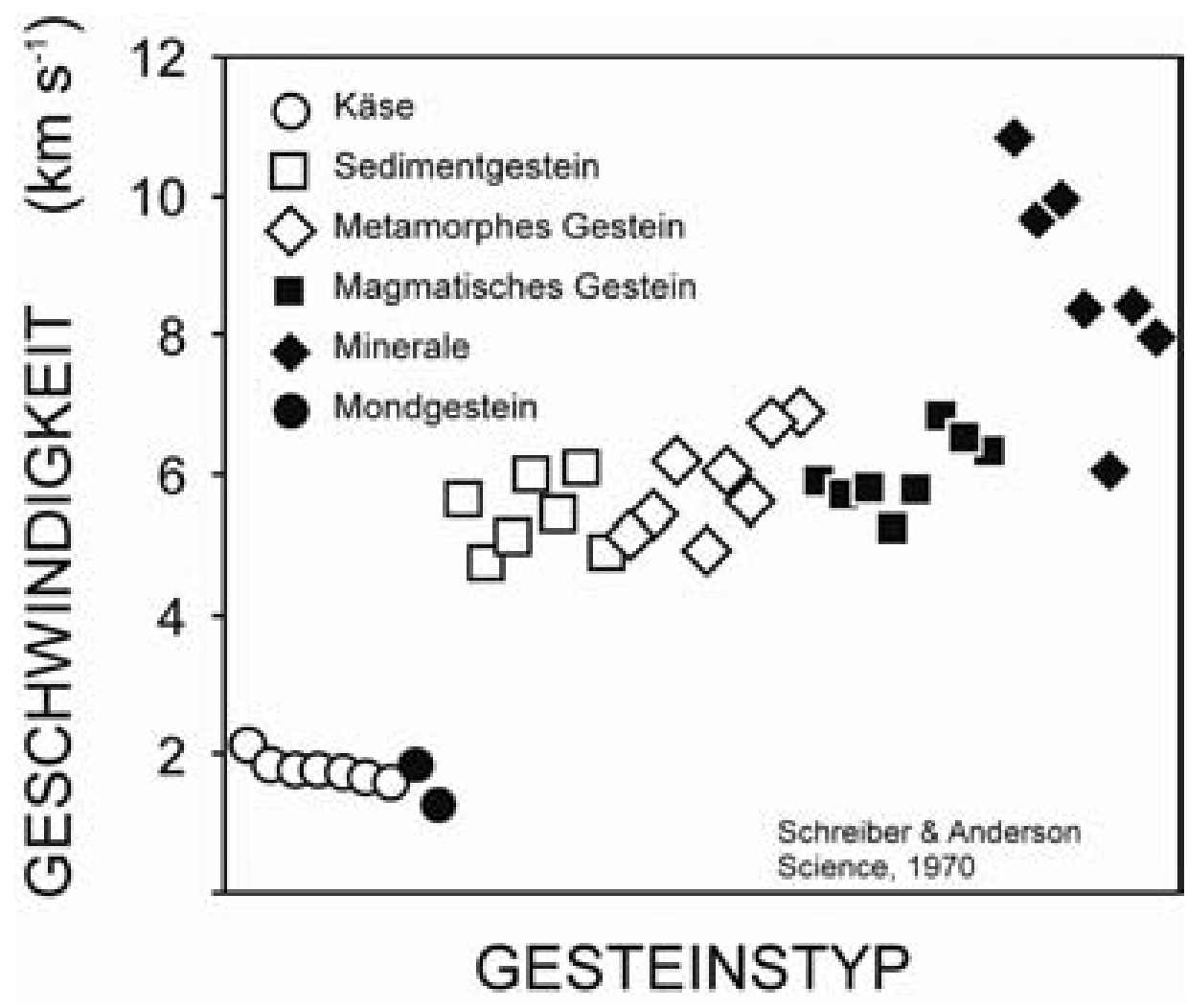

Abb. 1: Wellengeschwindigkeit von Mondgesteinen, unterschiedlichen Erdgesteinen und Mineralen und Käse (umgezeichnet aus Schreiber and Anderson, 1970). 
Um entscheiden zu können, ob eine Mineralart in der tiefen Erde existieren kann, vergleichen wir normalerweise Wellengeschwindigkeiten der Erde in der fraglichen Tiefe mit denen des Minerals im Labor bei entsprechendem Druck und der dazugehörigen Temperatur. Wie in Abb. 1 zu sehen ist, liegt die Wellengeschwindigkeit in verschiedenen Mineralen und Gesteinen der Erde zwischen 4,5 und $11 \mathrm{~km} \mathrm{~s}^{-1}$. Die Messungen am Mondgestein ergab eine Geschwindigkeit von $2 \mathrm{~km} \mathrm{~s}^{-1}$. Der dabei festgestellte Unterschied zwischen Erd- und Mondgestein würde bedeuten, dass diese nicht von denselben Mineralen aufgebaut werden.

Laut der philosophischen Vorgehensweise der Geowissenschaftler sollte das Mondgestein aus demselben Material sein, das dieselbe Wellengeschwindigkeit aufweist. Durchstöbert man die Literatur nach Vergleichsdaten, so findet man heraus, dass Käse dieselbe Wellengeschwindigkeit hat wie das Gestein unseres Trabanten. Glücklicherweise steht uns Mondgestein zur Verfügung, sodass wir mit eigenen Augen sehen können, dass die Minerale des Mondes mit denen der Erde identisch sind!

Was war dann falsch an den Messungen?

Das Mondgestein, das im Labor gemessen worden ist, hatte viele Risse zwischen den einzelnen Kristallen. Um Wellengeschwindigkeiten zu messen, wird eine Spannungswelle durch eine Probe geschickt. Die Zeit, welche die Welle braucht, um durch die Probe zu laufen, wird gemessen. Da die Welle bei dieser Probe auch durch die luftgefüllten Risse gehen musste - und die Geschwindigkeit durch Luft gerade einmal ein Zehntel dessen erreicht, welche in einem Feststoff erwartet wird - wird die durchschnittliche, gemessene Geschwindigkeit für die gesamte Mondprobe stark verringert, nämlich auf eine Durchlaufgeschwindigkeit von nur $2 \mathrm{~km} \mathrm{~s}^{-1}$. Da die Erdgesteine rissfrei gemessen worden sind, entstand die unerwartet hohe und inakzeptable Differenz zwischen den beiden eigentlich sehr ähnlichen Gesteinstypen! Um den verfälschenden Effekt der Risse zu eliminieren, mussten die Wissenschaftlicher die Mondproben erst unter Druck setzen, um die störenden Risse zu schließen. Dann werden auch die Geschwindigkeiten von ca. $5 \mathrm{~km} \mathrm{~s}^{-1}$ erreicht, wie wir sie schon von den Erdgesteinen kennen.

Auf diese Art und Weise versuchen wir, die Geheimnisse unseres Planeten zu lüften. Unsere Modellvorstellung basiert dabei auf den neugebildeten Gesteinen der mittelozeanischen Rücken, den Hot-Spot-Vulkanen und den Subduktionszonen-Vulkanen, sowie dem Altmaterial, das mit den Plattenrändern subduziert wird und der Geschwindigkeit und Dämpfung von seismischen Wellen in diesen Gebieten der Erde. Im Labor werden die Geschwindigkeit und die Dämpfung von Wellen in unterschiedlichen Gesteinen und Schmelzen gemessen. Diese Daten werden dann mit den seismischen Daten verglichen, um auszuwerten, ob das untersuchte Material in der Erde existieren darf. 


\section{Spannung, Verformung und Verformungsraten}

In unserem Planeten haben wir also Kristalle und Schmelzen. Um Messungen der Spannung und Verformung solcher Materialien machen zu können, müssen wir zuerst den Unterschied zwischen der elastischen Verformung eines Kristalls und der viskosen Verformung einer Schmelze verstehen. Wir haben alle einmal gelernt, dass Kristalle fest sind und auf Belastung elastisch reagieren. Wenn man eine konstante Spannung (Druck) auf einen Kristall ausübt, verformt er sich sofort. Diese Verformung bleibt erhalten, wenn die Spannung konstant aufrecht erhalten bleibt. Wird diese Art der Belastung weggenommen, so wird der Kristall sofort wieder seine ursprüngliche Ausgangsform annehmen. Übt man hingegen eine konstante Spannung auf eine Schmelze aus, gibt es keine sofortige elastische Verformung. Es kommt vielmehr zu einer zeitabhängigen, viskosen Verformung, und die Schmelze bleibt auch nach Wegfall der Spannung deformiert. Bei Kristallen wird also die für die Deformation nötige Energie zurückgewonnen, sobald die Spannung weggenommen wird. Bei der Formänderung der Schmelzen geht die zuvor aufgewendete Energie in der Deformation verloren und wird nicht wieder freigesetzt.

Mit dieser verhältnismäßig einfachen und traditionellen Lehrmeinung über das Verhalten von Kristallen und Schmelzen haben Geowissenschaftler inzwischen einige erhebliche Probleme. Das erste Problem hat mit dem Mantel zu tun: Der Mantel macht $85 \%$ des Erdvolumens aus. In ihm gibt es heißes Material, das in Form von Mantelplumes aufsteigt, und es gibt eintauchende tektonische Platten, die sich (in einem Zeitraum von Jahrmillionen) über Tausende von Kilometern bewegen können. Der kristalline Mantel ist kein starres Gestein, denn außer den oben angeführten Bewegungen anderer Materialien innerhalb des Mantels bewegt sich auch der Mantel selbst! Er konvektiert und ist damit auch eine Antriebskraft für die Plattenbewegungen der Kruste. Der Erdmantel ist kristallin. Und trotzdem bildet er in seinem Innern große Konvektionszellen, d. h. er fließt. Unsere bisherige Vorstellung von Kristallen war, dass sie fest sind, und dies erlaubt es eigentlich schon gar nicht, dass sie sich wie eine Flüssigkeit verhalten können.

Umgekehrt haben wir auch ein Problem mit den physikalischen Eigenschaften silikatischer Schmelzen, wie sie bei Vulkanausbrüchen auftreten. Bei heftigen pyroklastischen Ausbrüchen wird Magma fragmentiert. Obwohl aus flüssiger Schmelze bestehend, zerbricht es. Unsere Vorstellungen erlauben aber nur ein viskoses Verhalten, und die Zerbrechlichkeit ist eindeutig eine elastische Eigenschaft! Wie kann eine Schmelze zerbrechen?

Wir haben ein elegantes und sauberes Modell für das Erdinnere. Aber damit alles zusammenpasst - Modell und Beobachtung - muss es uns gelingen zu klären, wie Kristalle fließen und Schmelzen brechen können, und somit sich entgegen ihren erwarteten Eigenschaften verhalten können. Um unsere Modellvorstellungen und unsere Beobachtungen in Einklang miteinander zu bringen, müssen wir es zulassen, dass Erdmaterialien eigentlich viskoelastische Materialien sind. Es 
gibt kein Material, das nur elastische Eigenschaften hat und keines, das nur viskose Eigenschaften hat. Jedes Material - ob natürlich oder synthetisch - zeigt eine Mischung von beiden extremen Verhaltensweisen. Wir nutzen dies bereits aus, um Malerfarben, Zahnpasta, Kosmetika und Lebensmittel zu entwickeln. Abhängig von Druck und Temperatur kann sich ein Material mehr von seiner elastischen oder viskosen Seite zeigen. Wie verhalten sich nun viskoelastische Materialien, wenn eine konstante Kraft angelegt wird?

Wie in Abb. 2a gezeigt, erfolgt nach der Anlegung von Spannung die sofortige elastische Verformung und danach die zeitabhängige, anelastische Verformung. Beide sind energiespeichernd und somit reversibel. Danach gibt es die zeitabhängige, energieschluckende viskose Verformung. Abhängig davon, ob man unmittelbar nach Auflegung der Spannung die Verformung misst, oder eine Zeitlang wartet, um sie zu messen, misst man entweder die elastische oder die viskose Deformation. Es ist deshalb eine Frage der Zeit, welche der beiden Eigenschaften beobachtet werden kann - oder eine Mischung aus beiden!

Wir sehen diesen Effekt, wenn wir das elastische Modul und die Viskosität als Funktion der Zeit (oder der Frequenz) auswerten: Modul ist gleich Spannung geteilt durch Verformung. Wenn die konstante Spannung auf ein Material aufgelegt wird, gibt es eine sofortige elastische Verformung, und das resultiert in einem konstanten Wert des Moduls. Im Laufe der Zeit wird die Verformung durch die Anelastizität größer und das daraus kalkulierte Modul wird geringer. Nach einer sehr langen Zeit wird die viskose Verformung um einige Größenordnungen größer, als die elastische und anelastische Verformung. Wird eine konstante Spannung durch eine unendlich groß werdende Verformung geteilt, muß das kalkulierte Modul gegen Null gehen (Abb. 2b)!

Die Viskosität ist gleich Spannung geteilt durch Verformungsrate. Wenn die Spannung auf die Probe gelegt wird, kommt es zur sofortigen Verformung mit einer unendlichen Verformungsrate - und deswegen einer Viskosität von Null. Im Laufe der Zeit wird die Verformung durch den Effekt der anelastischen Deformation größer. Die kalkulierte Viskosität wird dabei immer größer, weil die Verformungsrate kleiner als unendlich wird. Nach einer längeren Zeit wird das viskose Fließen der einzige noch wirksame Verformungsprozess, der noch funktioniert, und die Verformungsrate nimmt einen konstanten Wert an. Somit wird auch die Viskosität konstant (Abb. 2c)!

Daraus wissen wir, dass der Mechanismus, der zur Verformung unseres Materials führt, davon abhängig ist, wie viel Zeit nach der Anlegung der Spannung vergangen ist. Obwohl ein Material viskoelastisch ist, könnte es passieren, dass wir nur die elastische oder nur die viskose Eigenschaft des Materials wahrnehmen. Bei einer Kurzzeitmessung messen wir also nur die elastischen Eigenschaften; die viskosen Eigenschaften können wir nur wahrnehmen, wenn wir den Messzeitraum erhöhen. 


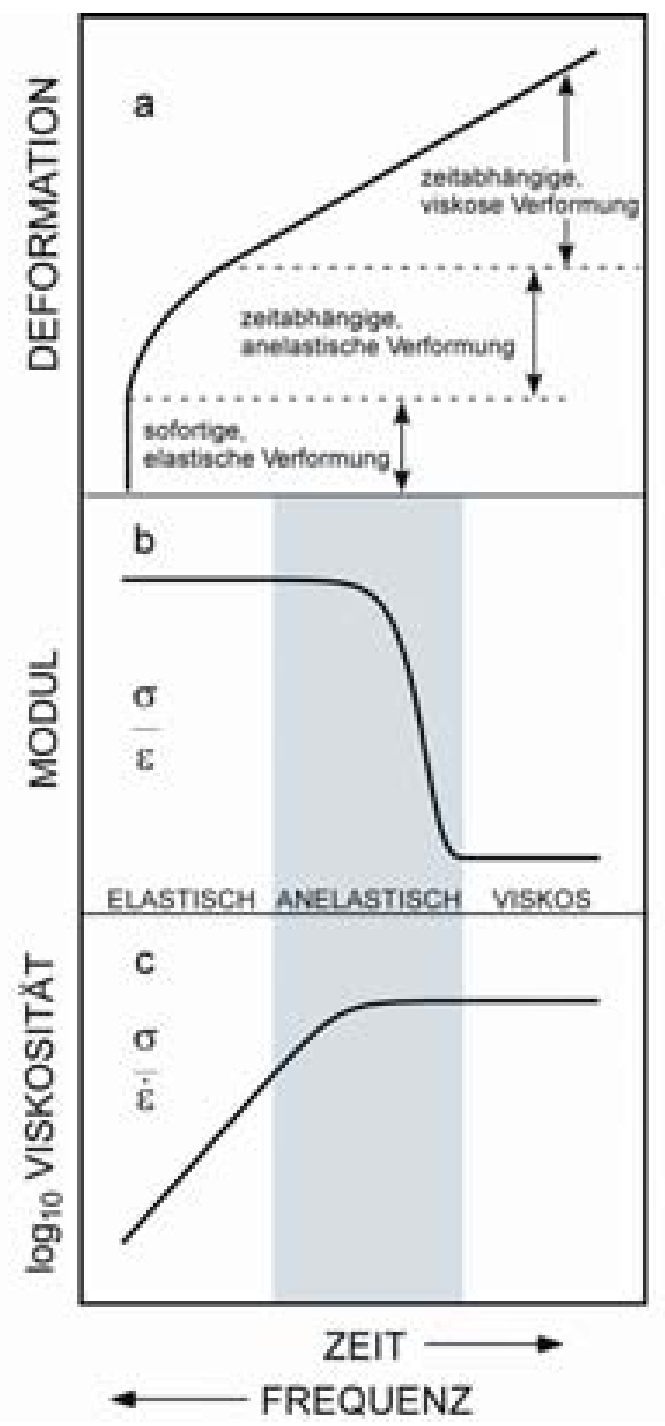

Abb. 2: (a) Verformung für eine konstante Spannung als Funktion der Zeit, zusammen mit (b) dem zeitabhängigen Modul und (c) der zeitabhängigen Viskosität.

Doch hat die Zeitabhängigkeit der physikalischen Eigenschaften von irdischen Kristallen und Schmelzen für uns überhaupt eine Bedeutung? Wir müssen noch einmal über unsere Messmethode nachdenken: Wir messen die Wellengeschwindigkeit von Proben im Labor. Es gibt eine einfache Beziehung zwischen der Frequenz der Spannungswellen, deren Geschwindigkeit und Wellenlänge. Um die Geschwindigkeit genau messen zu können, müsste die Probe mindestens zehn Wellenlängen groß sein. Die seismischen Wellen, welche die Erde durchlaufen und uns Auskunft über den Mineralbestand, Druck- und Temperaturbedingungen 
geben, haben eine Frequenz zwischen $10 \mathrm{~Hz}$ und $1 \mathrm{mHz}$. Bei einer durchschnittlichen Geschwindigkeit von $5 \mathrm{~km} \mathrm{~s}^{-1}$ hat die Welle eine Wellenlänge von 5 bis $50 \mathrm{~km}$. Das hieße, dass unsere Probenkörper mindestens $50 \mathrm{~km}$ lang sein müssten. So große Proben können wir nicht finden, geschweige denn, dass wir für sie genug Platz im Labor hätten! Unsere synthetischen Proben und die natürlichen Proben aus der Erde sind höchstens $5 \mathrm{~mm}$ lang. Um exakte Messungen zu machen, benutzen wir - dem Maßstab entsprechend - Ultraschallwellen in einem Frequenzbereich von 10 bis $100 \mathrm{MHz}$. Das entspricht einer Wellenlänge von $0,5 \mathrm{~mm}$ bis $50 \mu \mathrm{m}(1 \mathrm{~mm}$ entspricht $1000 \mu \mathrm{m})$. Es gibt einen Unterschied von 6 Größenordnungen zwischen der Frequenz einer seismischen Welle und der einer Ultraschallwelle.

Ist diese Frequenz-Anpassung an die Größe unserer Proben um sechs GröBenordnungen und deshalb der Zeit nach Anlegung der Spannung für unsere Messung von Verformung und Verformungsrate von Bedeutung und somit zulässig? Diese Frage lässt sich nur beantworten, indem wir die Labordaten für Wellengeschwindigkeiten und -dämpfungen in dem im oberen Mantel vorkommenden Mineral Olivin mit Messungen durch den oberen Erdmantel vergleichen. Die seismische Scherwellengeschwindigkeit für eine 1-Hz-Welle, die durch den Mantel reist, ändert sich mit zunehmender Tiefe (bis $600 \mathrm{~km}$ ) von $\sim 4,8$ bis $5,4 \mathrm{~km} \mathrm{~s}^{-1}$ (siehe Abb. 3a). Die im Labor gemessene Wellengeschwindigkeit für eine 10-MHz-Welle besitzt (innerhalb der Fehlertoleranzen) nahezu genau den gleichen Wert, wie wir ihn in der Erde selbst gemessen haben.

Scheinbar ist unsere Sorge um die Frequenzabhängigkeit der Eigenschaften unbegründet.

Wir versuchen, immer bessere und genauere Messmethoden zu entwickeln, um mehr über unser Universum herauszufinden. Inzwischen können wir die Dämpfung der Wellen messen - genauso, wie es die Seismologen tun. Für die Dämpfung $\left(\mathrm{Q}^{-1}\right)$ in der Erde wurde bei $1 \mathrm{~Hz}$ ein Wert von 0,006 gemessen. Im Labor wurde ein Wert von weniger als $10^{-4}$ für eine $10-\mathrm{MHz}-$ Welle ermittelt (Anderson, 1989). Diese Tatsache, dass die Geschwindigkeiten stimmen, aber die Dämpfung nicht, führt zu der brennenden Frage, ob unsere Labormessungen überhaupt realistisch sind und mit den seismischen Eigenschaften der Erde vergleichbar sind.

Hohe Dämpfung bedeutet ein größer werdender Einfluss der viskosen Eigenschaften. Die Konsequenz daraus ist, dass die hochfrequenten Ultraschallwellen nicht zur Erfassung des viskosen Fließens des Olivins geeignet sind und Messungen bei niedrigeren Frequenzen nötig sind.

Es gibt eine geniale Methode, Spannung und Verformung zu messen und daraus Wellengeschwindigkeit und Dämpfung zu kalkulieren - und das, ohne dass die Probe länger als $3 \mathrm{~cm}$ sein müsste. Bei dieser Torsions-Methode wird ein $\mathrm{Zy}$ linder mit einer sinusförmigen Spannung gedreht, und die daraus resultierende Verformung wird gemessen. Die Frequenz der Spannung variiert zwischen $1 \mathrm{mHz}$ und $10 \mathrm{~Hz}$. Die Dämpfung wird aus der Amplitude der Verformung berechnet. Wenn für eine angelegte Spannung die Verformung in Abhängigkeit von der Fre- 
quenz weniger wird, bedeutet das, dass die Dämpfung größer wird. Und somit wird auch der Anteil des viskosen Fließens größer - wie von der Theorie gefordert!

Der obere Erdmantel besteht zu 65 Volumenprozent aus dem Mineral Olivin. Deshalb haben Tan et al. (2001) eine Probe aus sehr feinen Olivinkristallen hergestellt und mit dieser Torsions-Methode gemessen. Wie in Abb. 3 zu sehen ist, sind die im Labor gemessenen Scherwellengeschwindigkeiten in Olivinen für Frequenzen zwischen 1 und $0,01 \mathrm{~Hz}$ (Wellenperiode von 1 bis 100 Sekunden) bei $1100{ }^{\circ} \mathrm{C}$ vergleichbar mit den seismischen Wellengeschwindigkeiten der Erde. Ebenso sind die beiden Dämpfungs-Werte des Laborexperiments und der Erde miteinander vergleichbar. Der Mantel hat jedoch in Tiefen von 200 bis $400 \mathrm{~km}$ eine Temperatur von $\sim 1400{ }^{\circ} \mathrm{C}$ (Jackson \& Rigden, 1998). Die im Labor gemessene Wellengeschwindigkeit in polykristallinem Olivin bei $1400{ }^{\circ} \mathrm{C}$ ist viel geringer als die seismische Geschwindigkeit; und die Dämpfung ist - im Gegensatz zu den Verhältnissen im Erdmantel - um den Faktor 200 größer.

Wir haben das richtige Gestein, den richtigen Druck, Temperatur, Frequenzen und Spannung. Was haben wir denn nun noch vergessen? Weil die Proben immer noch relativ klein sind, sind die Korngrößen der Minerale auch klein. In dieser Probe haben die Körner einen Durchmesser von 0,15 mm - und die SpannungVerformungs-Gleichung (Hirth \& Kohlstedt, 1995) ist korngrößenabhängig!

Die Dämpfung verhält sich zur Korngröße wie eine quadratische Funktion; d. h. einem Dämpfungsunterschied um den Faktor 200 steht ein Korngrößenunterschied mit dem Faktor 14 gegenüber. Für den Fall, dass wir eine Probe mit einer Korngröße von 2,1 mm für unsere Messung benutzen, bedeutet dies, dass wir dieselbe Scherwellengeschwindigkeit und Dämpfung messen werden, wie die von der Erde ermittelten Werte für seismische Wellen.

Doch wie groß sind die Olivin-Körner in $200 \mathrm{~km}$ Tiefe?

Hier kommt uns die Natur buchstäblich entgegen: Es gibt Vulkane, die OlivinKnollen mit an die Oberfläche bringen. Wenn die vulkanischen Ausbrüche heftig sind, kann das vorbeirauschende Magma Fragmente von den Wänden der Aufstiegsschlote aus bis zu $200 \mathrm{~km}$ Tiefe mit sich reißen. Die Körner in solchen Knollen sind 2-3 mm groß.

Das bedeutet, dass bei den niedrigen Frequenzen von seismischen Wellen, die durch die Erde reisen, die Kristalle genug Zeit haben, sich nach Anlegung der Spannung nicht nur elastisch, sondern teilweise auch viskos zu verformen. Und deswegen kann der Mantel konvektieren und fließen, obwobl er kristallin ist! In den Messungen von Tan et al. (2001) war der Beginn des Effekts des viskosen FlieBens der Olivinkristalle über einen Zeitraum von $10^{2} \mathrm{~s}$ zu beobachten. Die Verformung des Erdmantels vollzieht sich in einem Zeitraum von $10^{15}$ s. Dieser um 12 Größenordnungen differierende Zeitunterschied bedeutet, dass die $3 \mathrm{~mm}$ großen Olivinkörner im oberen Erdmantel ohne Probleme fließen können. Immerhin hat der Mantel viel Zeit - nämlich ein paar Millionen Jahre! Um das Problem des konvektierenden, kristallinen Mantels aus der Welt zu schaffen, müssen wir also nur einen viskoelastischen Mantel zulassen. 

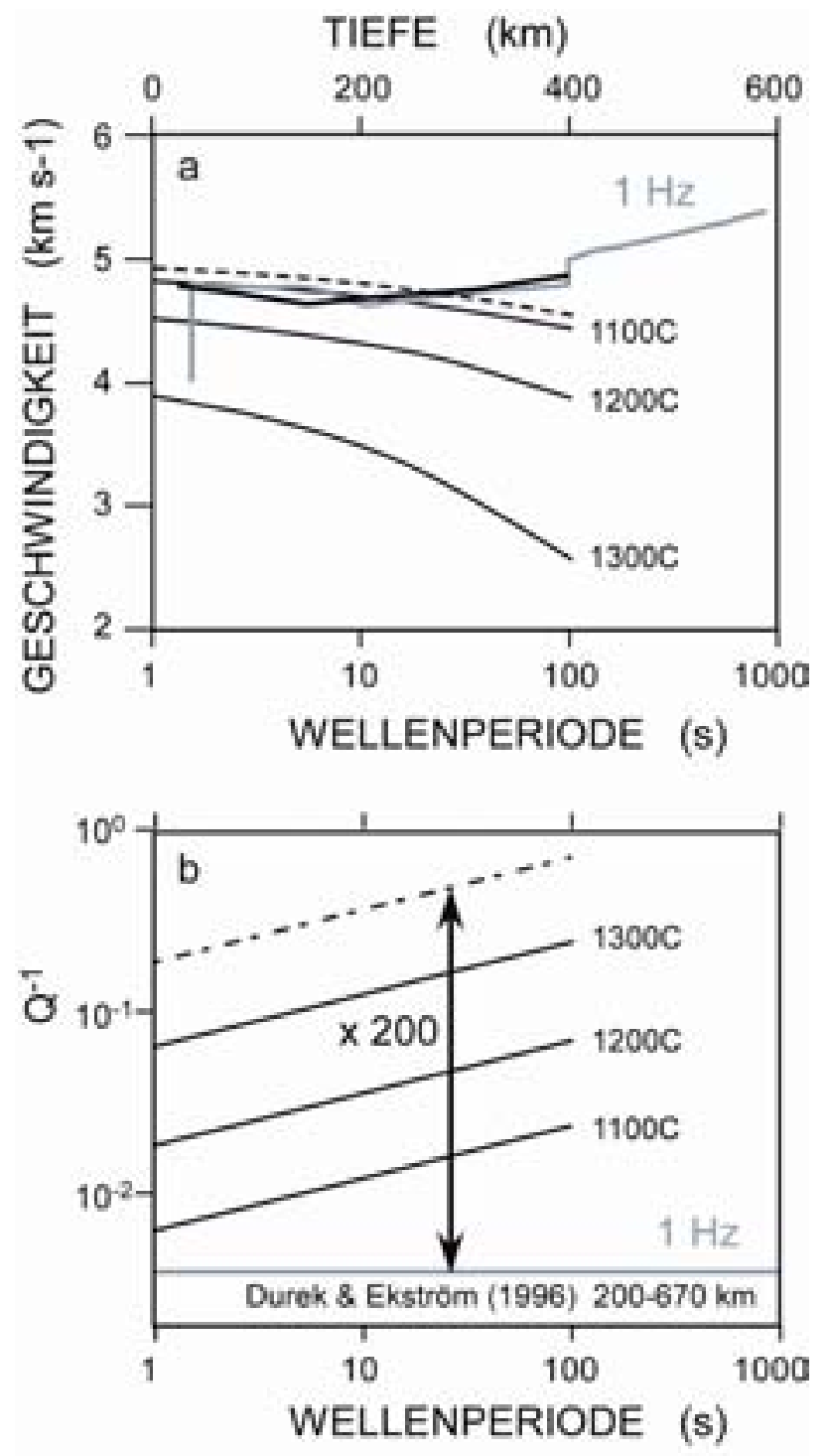

Abb. 3: (a) Geschwindigkeit in polykristallinem Olivin; graue Linie - seismische Daten für die Erde (Duffy \& Anderson, 1989); dicke schwarze Linie - Hochfrequenz-Ultraschalldaten von Duffy \& Anderson (1989); schwarze Linien - Hochtemperaturdaten von Tan et al. (2001) für seismische Frequenz-Labormessungen an Proben mit 0,15 mm Korngröße; gestrichelte Linie kalkulierte Geschwindigkeit für Olivin mit 2,1 mm Korngröße. (b) Dämpfung in polykeristallinem Olivin; graue Linie - seismische Daten für die Erde (Durek \& Ekström, 1996); schwarze Linien - Hocbtemperaturdaten von Tan et al. (2001) für seismische Frequenz-Labormessungen an Proben mit 0,15 mm Korngröße; gestrichelte Linie - extrapolierte Dämpfung für Olivin mit 0,15 mm Korngröße bei $1400^{\circ} \mathrm{C}$. 


\section{Fließende Kristalle}

Wie können Kristalle fließen? Wie jedes Material, auf das eine Belastung wirkt, wird auch der Kristall sich zuerst einmal sofort elastisch verformen. Wenn diese Spannung dann wieder weggenommen wird, nimmt der Kristall wieder seine Ausgangsform an. In dem Fall, dass die Spannung eine lange Zeit auf den Kristall wirkt, gibt es nicht nur die elastische Verformung, bei der jedes Atom in dem Kristallgitter ein bisschen verschoben wird. Einige der Atome können sich langsam aus dem Gitter lösen und sich durch Diffusionsprozesse zu einer Stelle mit niedrigerer Spannung bewegen. Falls das passiert, bevor die Spannung gewichen ist, wird der Kristall nicht zu seiner Ausgangsform zurückkehren, sondern bleibt dauerhaft verformt. In diesem Szenario ist elastische Energie in viskoses Fließen umgewandelt worden. Dieser Effekt zeigt sich bei der Messung als erhöhter Wert bei der Dämpfung, der bei einer rein elastischen Verformung geringer ist, als in dem gerade erwähnten Beispiel.

Aber wie fließt denn nun der Mantel? Welcher Mechanismus ist dafür verantwortlich, dass die tektonischen Platten sich bewegen und hierbei das Aussehen der Erdoberfläche in 50 Millionen Jahren bis hin zur Unkenntlichkeit verändert wird? Die Antwort auf diese Fragen lautet: durch die Bewegung der Atome! Ein Atom nach dem anderen wird durch die Kristalle des Erdmantels diffundieren und verleiht diesem damit die physikalischen Eigenschaften einer Flüssigkeit. Die langsame Bewegung der Atome in Nanometer-Schritten bewegt den 2900 km mächtigen Erdmantel!

\section{Hüpfende Schmelzen}

So, nun haben wir das erste Problem gelöst. Aber das zweite Problem ist immer noch da. Wie kann eine Schmelze zerbrechen? Warum zeigt eine Schmelze solche elastischen Eigenschaften?

Aus den physikalischen Eigenschaften für viskoelastische Materialien geht klar hervor, dass sich elastische Eigenschaften über sehr kurze Zeiträume zeigen. Im Gegensatz zu der Labor-Verformung von Kristallen - die normalerweise zu schnell ist, und deshalb nur elastische Eigenschaften gemessen werden - sind vielleicht die Messungen von Eigenschaften der Schmelze zu langsam, sodass nur das viskose Verhalten bisher wahrgenommen worden ist.

Wir haben uns Zylinder aus Silikatschmelzen hergestellt, und die SpannungsVerformungs-Verhältnisse als Funktion der Zeit (Frequenz) gemessen. Hierzu haben wir uns derselben Torsions-Methode bedient, wie sie auch schon bei den viskoelastischen Kristallen angewandt worden ist. Die Messungen wurden im seismischen Frequenzbereich durchgeführt. Die gemessene Viskosität ist, wie von der Theorie vorhergesagt, frequenzabhängig (Webb, 1992). Silikatische Schmelzen sind viskoelastische Materialien! Ob sie elastische oder viskose Eigenschaften zei- 
gen, ist von der Wirkungsdauer der Spannung abhängig. Nach längerer Einwirkung einer Spannung oder bei einer Druckänderung in einer Magmakammer, welche die Eruption provoziert, verhält sich die Schmelze wie ein viskoses Material. Aber in dem kurzen Millisekundenbereich nach der Druckwelle, welche die Eruption vorantreibt, benimmt sich die Schmelze wie ein elastischer Festkörper.

Wie erwartet, ist auch das gemessene elastische Modul abhängig von der Zeit und damit der Frequenz der sinusförmigen Spannung. Der vulkanische Ausbruch wird durch den plötzlichen Druckstoß angetrieben. In der kurzen Zeit nach jeder solchen plötzlichen Spannungsänderung benimmt sich die Schmelze zuerst wie ein elastisches Material und zeigt die physikalischen Eigenschaften, wie man sie von einem Festkörper erwartet. Dies bedeutet, dass auch eine Schmelze zerbrechlich sein kann und wie ein Stück Fensterglas gebrochen werden kann. Dazu ist es nötig, dass die Spannung schnell genug angelegt wird. Dies passiert zum Beispiel bei einem pyroklastischen Ausbruch! Sprödheit ist nicht die einzige elastische Eigenschaft, die silikatische Schmelzen zeigen: In den Millisekunden nach Anlegung der Spannung können Schmelzen hüpfen und hopsen und, wie jedes andere elastische Material auch, zurückspringen.

Nun ist auch das zweite Problem mit den physikalischen Eigenschaften der Erdmaterialien geklärt. Silikatische Schmelzen sind spröde und werden während gewaltiger pyroklastischer Ausbrüche zerbrochen. Das passiert, weil geschmolzene Steine eben auch viskoelastische Materialien sind.

Wie fließt eine silikatische Schmelze? Welche strukturelle Besonderheit führt zur viskoelastischen - und somit zeitabhängigen - Änderung im Verformungsmechanismus einer Schmelze? Eine silikatische Schmelze ist ein dreidimensionales Bauwerk, das durch Si-O-Si-Bindungen zusammengehalten wird. Jede dieser Bindungen hat eine gewisse Lebenszeit. Nach dieser Zeit wird die Bindung gelöst, und das Si-Atom sucht sich einen neuen Si-Partner. Falls kein neues Si-Atom in der Lebenszeit einer Bindung gefunden wird, wird das Si wieder eine Bindung mit dem Original-Partner eingehen. Die Struktur ist dynamisch und ändert sich laufend, aber die durchschnittliche Anzahl von Si-O-Si-Bindungen bleibt konstant. Im Innern der Schmelze sieht es aus wie bei einem Walzer. Die tanzenden Si-OSi-Komplexe bleiben einige Zeit zusammen, dann gehen sie auseinander, um neue Partner zu suchen. Falls keine neuen da sind, gehen die beiden Tänzer wieder zueinander zurück und tanzen noch eine Weile zusammen. Während das passiert, ist die ganze Gruppe immer in Bewegung.

Was verursacht solche tanzenden Si-Atome in einem viskoelastischen Material? Wenn eine Spannung aufgelegt wird, verformt sich die Schmelze, und die Verbindungen zwischen den Atomen reagieren zuerst nur elastisch. Dann, im Laufe der Zeit, werden Si-O-Si-Bindungen zerbrochen und neue Partner gesucht, die am Anfang nicht gefunden werden. Wird die Spannung jedoch konstant aufrecht erhalten, so finden die Si-Atome nach langer Zeit doch neue, freigewordene Partner und gehen neue Bindungen ein. Durch diesen Prozess fließt die Schmelze. Nur wenn genug Zeit vergangen ist und die Si-Atome neue Tanzpartner bekommen, fließt die Tanzgruppe. Das ist die Beschreibung eines schön ruhig- 
fließenden Magma-Ausbruchs, wie auf Hawaii, bei dem ein wirbelnder Fluss von tanzenden Si-Atomen sich über die Erdoberfläche wälzt.

Wenn aber die Spannung in der Schmelze sehr schnell geändert wird, wie bei einem explosiven, pyroklastischen Ausbruch, müssen sich die Si-Atome sehr schnell bewegen. In dieser kurzen Zeit können die Si-Tänzer die Bindung zum alten Partner nicht auf die natürlich zeitintensive Art und Weise lösen und wieder aufnehmen, sondern die Bindungen werden mit Gewalt aufgebrochen und die ganze Schmelze (Tanzgruppe) fällt auseinander in kleine, spröde Glasfragmente.

\section{Zusammenfassung}

Auf den ersten Blick erscheint uns unser Zuhause als ein schöner, blauer und vor allem friedlicher Planet, auf dem es sich bequem leben lässt. Aber unter unseren Füßen bewegen sich die tektonischen Platten, Kruste wird subduziert, der kristalline Mantel konvektiert, und es gibt immer wieder aufsteigende Plumes, die heiße Minerale und geschmolzenes Gestein zutage fördern. Dieses Bild vom groBen Ganzen wurde mithilfe der Geschwindigkeit und der Dämpfung von seismischen Wellen, die durch die Erde reisen, aufgenommen.

Welche Kraft und welcher Mechanismus treibt das ganze Brodeln an? Die Kraft ist die Spannung, und der Mechanismus ist der dynamische, immer wechselnde Strom von sich bewegenden Atomen, die unter der trügerisch-ruhigen Oberfläche des Planeten nie zur Ruhe kommen. Ob nun diese Atome im Kristallgitter sitzen oder in Schmelzen tanzen, sie sind es, die die Erdmaterialien unter unseren Füßen aufbauen und das viskoelastische Verhalten der unterschiedlichsten Materialien unseres Planeten bewirken.

Was bewegt die Erde? Atome, die unterwegs sind, bewegen die Erde. Tanzende Atome und Atome, die eine spannungsfreie Stelle suchen, machen Ångströmgroße Schritte, um die Welt in Gang zu setzen. Als Resultat dieser kleinen Schritte können wir farbenfrohe vulkanische Ausbrüche bewundern und aufregende Erdbeben erleben.

\section{Danksagung}

Ich möchte mich bei Gerrit Raschke für die Korrektur der Grammatik und bei Dr. Andreas Kronz für die hilfreichen Verbesserungsvorschläge herzlich bedanken. 


\section{Literatur}

Anderson, D. L. (1989): Theory of the Earth, Blackwell

Duffy, T. S. and Anderson, D. L. (1989): Seismic velocities in mantle minerals and the mineralogy of the upper mantle, J. Geophys. Res., 94, 1895-1912

Durek, J. J. and Ekström, G. (1996): A radial model of anelasticity consistent with long-period surface-wave attenuation, Bull. Seismol. Soc. Amer., 86, 144-158

Jackson, I. and Rigden, S. M. (1998): Composition and temperature of the Earth's Mantle: Seismological Models Interpreted through Experimental Studies of Earth Materials. In. The Earth's Mantle, I. Jackson (editor), Cambridge University Press

Hirth, G. and Kohlstedt, D. L. (1995): Experimental constraints on the dynamics of partially molten upper mantle: Deformation in the diffusion creep regime, J. Geophys. Res., 100, 1981-2001

Schreiber, E. and Anderson, O. L. (1970): Properties and composition of lunar materials; earth analogies, Science, 168, 1579-1580

Tan, B. H., Jackson, I. and Fitz Gerald, J. D. (2001): High-temperature viscoelasticity of fine-grained polycrystalline olivine, Phys. Chem. Mineral., 28, 641-664

Webb, S. L. (1992): Low-frequency shear and structural relaxation in rhyolite melt, Phys. Chem. Mineral., 19, 240-245 


\title{
Isotope, die Gene der Gesteine
}

\author{
Bent Tauber Hansen
}

Der oben aufgeführte Titel wird manche dazu veranlassen, sich zu fragen, wo denn der Zusammenhang zwischen Genen und Isotopen besteht. Der Vergleich ist nahe liegend. Betrachtet man die menschliche Zelle, hat diese einen Zellkern mit Chromosomen, in denen die individuellen DNA-Informationen enthalten sind. Bei den Gesteinen entsprechen die einzelnen Kristalle sozusagen den Zellen, und das Kristallgitter ist mit dem Zellkern zu vergleichen. Im Kristallgitter sind durch Einbau von radiogenen und radioaktiven Isotopen Informationen gespeichert, die für diesen Kristall und somit letztlich für das betreffende Gestein einzigartig sind. Um diese Informationen verstehen zu können, müssen wir erst zwei Begriffe definieren, „Isotope“ und „radioaktiven Zerfall“.

Die natürlichen Elemente (die 92 chemischen Elemente des Periodensystems von Mendelejew, 1869) sind eine Mischung von Isotopen, d. h. von Atomen, deren Kerne dieselbe, für ein Element bezeichnende Anzahl von Protonen enthalten und die sich nur in der Zahl der Neutronen unterscheiden. Ein Isotop wird durch die Gesamtzahl von Protonen und Neutronen gekennzeichnet, die man oben links vor das chemische Symbol schreibt. Einige an Neutronen reiche Isotope haben instabile Kerne. Diese neigen spontan dazu, ihre Energie zu verringern, indem sie etwas abgeben, z. B. ein Alpha-Teilchen (zwei Protonen und zwei Neutronen), die anderen ein Elektron (oder Beta-Teilchen), das durch Spaltung eines Neutrons in ein Proton und ein Elektron entsteht. Sie verwandeln sich somit in so genannte „Tochterelemente“, deren Kern eine (vom Mutterelement) abwei- 
chende Zahl von Protonen enthält. Das ist der Prozess des radioaktiven Zerfalls. Die Tochterelemente können ihrerseits selbst radioaktiv sein und zerfallen entsprechend weiter, bis am Ende ein stabiler Kern entstanden ist.

Der Prozess des radioaktiven Zerfalls ist seit über hundert Jahren bekannt. Im Jahre 1896 veröffentlichte Henry Becquerel in den „Comptes rendus de l'Académie des sciences“ die Ergebnisse seiner Versuche „sur les radiations invisible émises par les corps phosphorescents" (Über die von phosphoreszierenden Körpern ausgesandten unsichtbaren Strahlen, Becquerel 1896). Das Phänomen der spontanen Emission von Strahlung, das Marie Curie „Radioaktivität“ getauft hatte, ist einige Jahre später von Rutherford und Soddy (1902a, 1902b) erklärt worden. Diese Autoren haben auch die Gesetzmäßigkeit des radioaktiven Zerfalls beschrieben und somit die Grundlage geschaffen, Datierungen z. B. an Gesteinen, die radioaktive Elemente enthalten, vorzunehmen. Zunächst widmeten sich die Forscher dem radioaktiven Zerfall von Uran und Thorium. Dabei fiel es dem amerikanischen Chemiker Richards auf, dass Blei, das durch radioaktiven Zerfall entstanden war, ein anderes Atomgewicht hatte als das des gewöhnlichen Bleis (Richards und Lembert 1914). Dieses Problem wurde durch eine kühne Behauptung Soddys gelöst, indem er sagte, dass ein spezifisches Element mehr als eine Art von Atomen besitzt. Er nannte diese Atome „Isotope“ aus dem griechischen „gleicher Platz". Diese Theorie wurde 1913 durch die Beobachtungen von Thomson bestätigt, denn er konnte nachweisen, dass Neon aus zwei verschiedenen Atomen mit unterschiedlichem Gewicht besteht.

Die Erdwissenschaftler haben sich seit jeher mit der Frage des Alters der Erde beschäftigt, doch war diese Frage bis Mitte des achtzehnten Jahrhunderts eine Frage der Theologen. Die Erde wurde am 23. Oktober 4004 vor Christus um 9 Uhr vormittags erschaffen. So hatte es James Ussher, anglikanischer Erzbischof von Armagh in Irland, 1654 anhand gründlicher Forschungen in der Bibel berechnet. Laut Bischof Ussher wäre die Erde 6400 Jahre alt, wenn man in Rechnung stellt, dass das Jahr Null in der Zeitrechnung nie existiert hat. Usshers Berechnungen für den Schöpfungszeitpunkt der Welt und andere biblische Ereignisse sind in den Seitenüberschriften der „King James Bible“ angegeben (Ältere jüdische Berechnungen geben als Schöpfungsdatum den 7. Oktober 3761 vor Christus an). Wie Erzbischof Ussher nebst Datum auch auf die Uhrzeit der Schöpfung gekommen ist, bleibt ein Rätsel. Immerhin ist die Sonne laut Bibel erst am vierten Tag erschaffen worden. Es erhebt sich also die Frage, wie lange die ersten drei Tage gedauert haben. Doch nicht nur Bischof Ussher, sondern auch der Theologe Burnet beschäftigte sich mit der Bildung der Erde. In seinem Buch „Telluris theoria sacra“ (Abb. 1) veröffentlichte er sein „Eierschalen-Modell“ für die Entstehung der Gebirge (Burnet 1681). Die Altersabschätzungen von Ussher blieben unumstritten, bis Hutton 1785 in seinem Buch „Theory of the Earth“ die Grundlagen eines modernen geologischen Entwicklungsprozesses publizierte. Er hielt somit als Erster das Alter der Erde auf Grund seiner Überlegungen zur Bildung mächtiger Sedimentformationen für viel älter als bis dahin angenommen. 


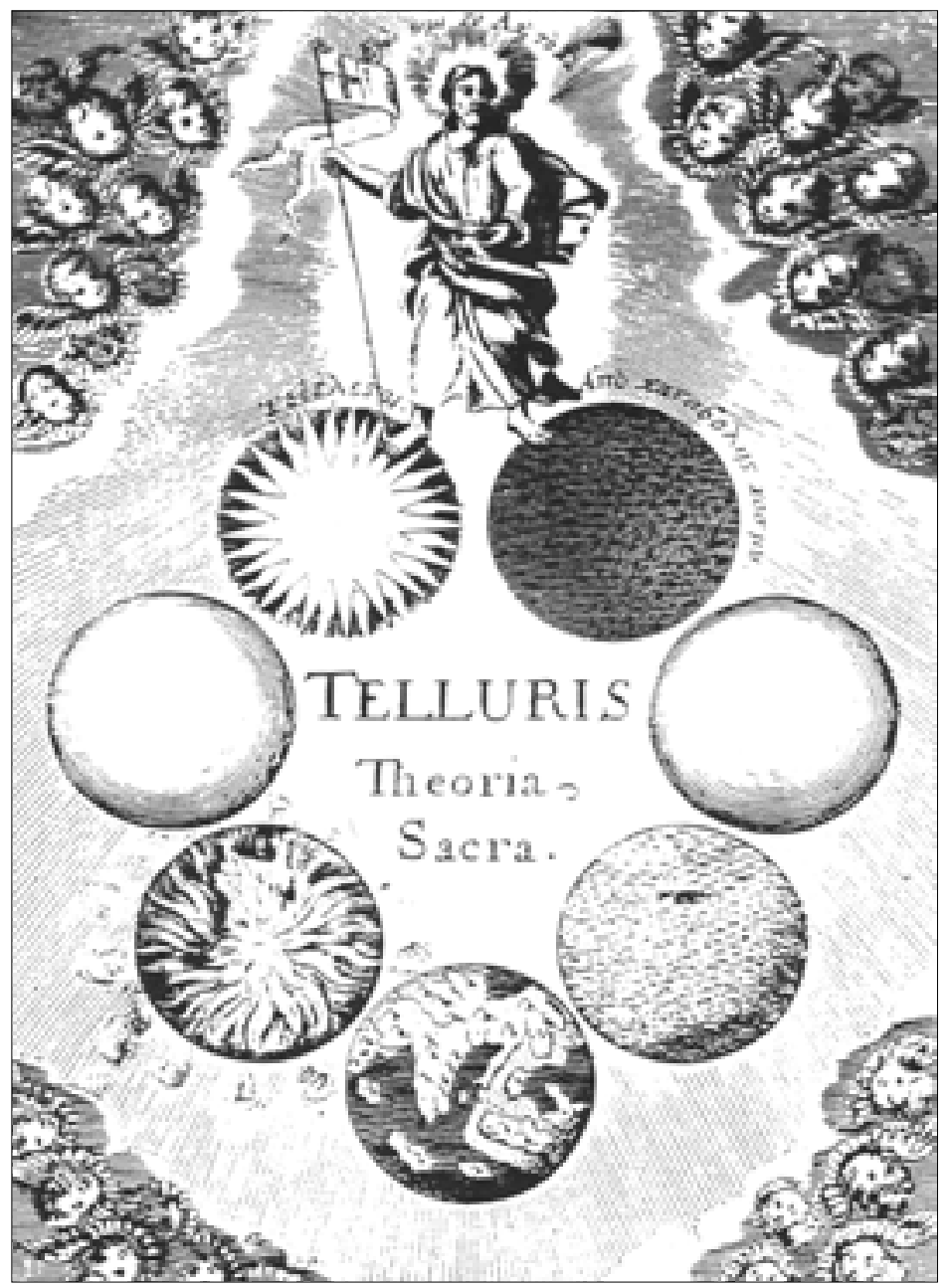

Abb. 1: Burnet (1681), Telluris theoria sacra.

Im Jahre 1830 publizierte Charles Lyell seine „Principles of Geology“, damit war die Grundlage für eine neue Generation von Geologen geschaffen, die von nun an nicht mehr verlangten, dass die geologischen Entwicklungstheorien mit den Büchern Moses in Einklang sein müssten und dass die Erdoberfläche durch die Sintflut geschaffen wurde.

Die modernen Isotopenuntersuchungen und somit die ersten Altersbestimmungen an Gesteinen wurden erst nach Entwicklung des modernen Massenspektrometers (Nier 1940) möglich. Nach dem Zweiten Weltkrieg erschienen die ersten Publikationen zur Altersbestimmung an geologischem Material (z. B. Aldrich und Nier 1948). 
Der größte Fortschritt entstand im Zusammenhang mit den amerikanischen Apollo-Missionen zum Mond (1969-1972). Diese Herausforderung an die Erdwissenschaftler und Physiker brachte die Technik der Altersdatierung entscheidend nach vorn. Nicht nur die Gesteine des Monds wurden mit großem Aufwand isotopisch beschrieben und datiert, gleichzeitig versuchten Wissenschaftler, die ältesten Gesteine der Erde zu entdecken, um die Theorien eines gemeinsamen Ursprungs von Erde und Mond zu beweisen. Hierbei wurde zunächst die Arbeitsgruppe aus Oxford bekannt, als es ihr gelang, Gesteine aus West-Grönland mit einem Alter von 3,7 Milliarden Jahren zu datieren (Black et al. 1971). Die Faszination der Geochronologen bei der Suche von Archaischen Gesteinen hat sich bis heute gehalten. Unter Inanspruchnahme von immer ausgereifteren Techniken ist es mit Hilfe einer hochauflösenden Ionensonde gelungen, Orthogneise von der Insel Akilia (SW-Grönland) mit einem Alter von mehr als 3,8 Milliarden Jahren zu datieren (Mojzsis \& Harrison 2002). In einem Zirkon aus diesem alten Krustensegment wurde sogar ein Alter von 4,1 Milliarden Jahren gemessen.

Die Isotopenuntersuchungen lassen nicht nur Aussagen über das Alter einer Gesteinsformation zu, sondern tragen auch zur Klärung des tektonischen Aufbaus ganzer Gesteinsformationen bei.

Der dänische Gelehrte Niels Stensen, besser bekannt unter seinem lateinischen Namen Nicolaus Steno (1638-1686) hat sehr früh den Begriff Strata geprägt. Ein wesentlicher Punkt in Stenos Theorie war, dass in einer Gesteinsfolge die jüngsten Gesteine als oberste Schichten zu finden sind und dem zu Folge die Schichten, je tiefer sie liegen, älter werden. Diese Theorie basierte auf der Annahme, dass alle Gesteine sedimentären Ursprungs sind. Diese und viele andere Theorien zu mineralogischen und geologischen Beobachtungen publizierte Steno in seinem legendären Buch „Prodomus“ (1669) kurz nach seiner Konvertierung zum katholischen Glauben (1667). Danach widmete er sich ausschließlich Glaubensfragen. Dennoch blieb das von ihm geprägte „Überlagerungs-Prinzip“ die gängige Interpretation, bis die modernen Datierungsmethoden es ermöglichten, nicht Fossil führende Gesteine zu datieren und somit Gesteinsfolgen mit inversen Altersabfolgen zu erkennen. Ein typisches Beispiel hierfür ist z. B. die Datierung der „Klippe von Omkoi“" in NW-Thailand, wo mit Hilfe von absoluten Altersbestimmungen zwei Überschiebungen von älteren Gesteinspaketen auf die jüngere Unterlage nachgewiesen werden konnte (Ahrendt et al. 1997). Eine typische Anwendung von Altersbestimmungen zum Nachweis von tektonischen Bewegungen in niedrig-metamorphen Gesteinen sind z. B. die Datierungen von großräumigen Überschiebungen in Vietnam (Wemmer et al. 1999).

Wie wir bereits gesehen haben, geben die Isotope nicht nur Auskunft über das Alter, sondern sind auch charakteristisch für die einzelnen Gesteine. Wenn diese Gesteine durch Verwitterung zu Böden umgewandelt werden, wird zwar die Konzentration einzelner Elemente teilweise verändert, aber eine Fraktionierung der Isotopie findet zunächst nicht statt, zumindest ist auch für den Boden die isotopische Zusammensetzung charakteristisch. Die Isotopie des Bodens wird über die Pflanzen aufgenommen und gelangt letztendlich somit in die Nahrungskette. 
Da sich die Bevölkerung im Mittelalter ausschließlich mit heimischen Nahrungsmitteln versorgte, wurde die Isotopie der Böden des jeweiligen Lebensraumes letztendlich in den Körpern der dort lebenden Menschen gespeichert. Da die chemischen Eigenschaften (Ionenradius und Ladung) von Calcium denjenigen von Strontium sehr ähnlich sind, wird im menschlichen Hartgewebe anstelle von Calcium Strontium eingebaut. Weit über $90 \%$ des im menschlichen Körper enthaltenen Strontiums ist im Skelett gebunden (Schroeder et al. 1972). Menschlicher Knochen wird ständig regeneriert, sodass ein kompakter Oberschenkelknochen (Femur) z. B. Strontium enthält, das ein Mensch in den letzten fünf bis zehn Lebensjahren aufgenommen hat (Glimscher 1976). Das im menschlichen Femur fixierte Strontium weist daher die lokale Strontium-Isotopensignatur des Lebensraums eines Individuums in den maximal letzten zehn Jahren auf. Der Zahnschmelz eines Menschen stellt nach seiner endgültigen Bildung im Gegensatz zum Knochen nahezu zellfreies und damit nicht stoffwechselaktives Gewebe dar, dass im weiteren Lebensverlauf unverändert bleibt. Die Krone eines ersten permanenten Backenzahns (1. Dauermolar) ist im Durchschnitt mit drei Jahren ausgebildet, wobei die Streuung \pm 12 Monate beträgt. Der Zahnschmelz eines 1. Dauermolaren enthält Strontium, das ein Individuum in den ersten vier Lebensjahren aufgenommen hat (Grupe et al. 1997). Somit hat es die lokale Strontium-Isotopensignatur der Region gespeichert, in der der betreffende Mensch seine ersten vier Lebensjahre verbracht hat. Durch den Vergleich der ${ }^{87} \mathrm{Sr} /{ }^{86} \mathrm{Sr}-$ Verhältnisse im Femur und im Zahnschmelz eines Individuums kann unter Umständen ein Ortswechsel zu Lebzeiten überprüft werden. Im Rahmen einer wissenschaftlichen Zusammenarbeit zwischen dem Geowissenschaftlichen Zentrum der Universität Göttingen, dem Anthropologischen Institut der Universität Göttingen und dem Institut für Ur- und Frühgeschichte der Universität Freiburg wurden Skelettproben von der Ausgrabung „Geißmatte“ bei Sulzburg im Breisgau auf ihre Strontium-Isotopie untersucht. Dieser Friedhof gehörte vermutlich zu einer Siedlung, deren Gründung hauptsächlich auf den in der Region betriebenen Silberbergbau zurückgeführt wird. Damit konnte eine Identifizierung der Individuen erfolgen, die zu Lebzeiten in das Arbeitsgebiet eingewandert waren. Dadurch ist ein Beitrag zur Klärung der Frage geleistet worden, ob im mittelalterlichen Bergbau der Region im 12. und frühen 13. Jahrhundert heimische Bergleute tätig waren, oder ob diese aus anderen Regionen nach Sulzburg eingewandert sind (Wormuth et al. 2000, Schutkowski et al. 2001).

Wie die Isotopensignaturen in den menschlichen Körper bzw. in das Hartgewebe des Menschen gelangen, so werden auch die entsprechenden Signaturen über die Nahrungsaufnahme von Kühen letztlich in die Milch und somit in die Butter gelangen. Diese Tatsache verhilft heute Zollfahndern dazu, falsch deklarierte Butter und die damit erschwindelten EU-Subventionen zu entlarven, denn schließlich besitzt Butter aus der Alpenregion eine andere Isotopensignatur als Butter aus Polen oder Neuseeland (Rossmann et al. 2000).

Ebenfalls verhilft diese Technik auch zur Identifikation der Herkunft von Weinen bzw. ob diese durch Verschnitt verfälscht wurden (Horn et al. 1998). 
Da es mit Hilfe moderner Isotopenanalytik wie gezeigt möglich ist, Wirtschaftskriminellen ihre Vergehen nachzuweisen, war es nahe liegend, den Versuch zu unternehmen, auch historische Kriminalfälle mit Messungen von Isotopenverhältnissen zu lösen. Der schwedische König Karl XII. (1682-1718) wurde 1718 in der Schlacht bei Fredrikshald in Norwegen aus dem Hinterhalt erschossen. Seitdem wird gerätselt, ob der König von einem norwegischen Feind erschossen wurde oder ob nicht vielmehr von einem Soldaten aus den eigenen Reihen, da die Angehörigen der schwedischen Armee des neunzehn Jahre andauernden Krieges längst müde waren. Um die These zu untersuchen, wurde die vermeintliche Kugel (ein mit Blei gefüllter Uniformknopf) auf ihre Blei-Isotopie untersucht. Dieser Knopf befindet sich zusammen mit der Uniform, die der König bei seiner Ermordung trug, im Museum von Varberg (Schweden). Man erhoffte sich aus der Zusammensetzung der Blei-Isotope Informationen über die Herkunft des Bleis und somit, ob es sich um norwegische oder schwedische Munition gehandelt hatte. Die Isotopenverhältnisse stimmen zwar recht gut überein mit denen des Erzes aus dem sogenannten Oslofeld in Norwegen, sind aber dem des Bleis aus nordeuropäischem Kupferschiefer noch ähnlicher (Wedepohl et al. 1978). Somit kann eine Herkunft durch Import entweder nach Norwegen oder nach Schweden nicht ausgeschlossen werden. Damit bleibt die Frage nach der Nationalität des Schützen ungelöst.

Die Frage nach der Blei-Isotopenzusammensetzung in Munition ist heute nach wie vor bei der Aufklärung von Verbrechen von großer Bedeutung. Die Firma Winchester lässt täglich ihre Produktion auf Blei-Isotopie untersuchen (Andrasko et al. 1993) und anhand der Auslieferungslisten können somit am Tatort sichergestellte Projektile zum Verkäufer zurückverfolgt werden.

Nicht nur die Bleizusammensetzung in Projektilen dient zur Überführung von Kriminellen, auch andere Isotope wie z. B. Sauerstoff hinterlassen deutliche „Fingerabdrücke“.

Zwischen Dezember 1993 und Dezember 1996 sorgten in Österreich und Deutschland vier Serien von Briefbomben an Prominente, darunter den damaligen Bürgermeister Helmut Zilk, für Aufregung. Da der Täter äußerst geschickt seine Spuren verwischte, hatten die ermittelnden Behörden lange Zeit keine Anhaltspunkte. Durch einen Artikel in „Der Spiegel“ wurde das deutsche Bundeskriminalamt auf die Arbeitsgruppe um Dr. H. Förstel am Forschungszentrum Jülich aufmerksam. In seiner Arbeitsgruppe werden die Isotopenverhältnisse von Sauerstoff in Wasserproben analysiert. Diese Daten erlauben es flächendeckend die regionale Herkunft von Wasser zu bestimmen. Da solche Analysen auch für Österreich verfügbar waren, war es nun möglich, den Täter regional einzugrenzen. Das Wasser wurde aus dem Gipsfuß einer Rohrbombe gewonnen, die aus dem Anschlag auf eine Roma-Siedlung im österreichischen Oberwart, bei der vier Menschen getötet wurden, stammte. Die Untersuchungen der Jülicher Gruppe bestätigten die Vermutung der österreichischen Behörden, dass der Täter aus dem Süden Österreichs, höchstwahrscheinlich aus dem südlichen Burgenland oder der Süd-Steiermark kam, konnten dagegen Wien und alle Gebiete nördlich der Alpen 
ausschließen (Messer Griesheim 2002). Hiermit wurde ein wesentlicher Beitrag zur Überführung des Bombenlegers Franz Fuchs mit Hilfe der Isotopenanalytik geleistet.

Als der Meteorologe Henrik Mohn (1835-1916) aus der Anlandung von sibirischem Treibgut an der Küste von Südwest-Grönland auf eine von Landmassen nicht gestörte Westdrift im Polarmeer schloss, fühlte der norwegische Polarforscher Fridtjof Nansen (1861-1930) sich herausgefordert, diese These zu untermauern.

Mit der finanziellen Unterstützung des norwegischen Parlaments, des Königs und privaten Spendern ließ er in Larvik von dem Schiffsbauer Collin Archer ein dem Packeis besonders angepasstes Schiff bauen, die 307 t große „Fram“ („Vorwärts“). Das Schiff hatte eine Wasserverdrängung von 800 Tonnen, eine Länge von $39 \mathrm{~m}$, eine Breite von $11 \mathrm{~m}$ und einen Tiefgang von $5 \mathrm{~m}$. Der Rumpf war so stark gebaut und so geschickt geformt, dass er, eingefroren im Packeis, nicht wie viele andere Schiffe vorher von den Eismassen zerdrückt wurde.

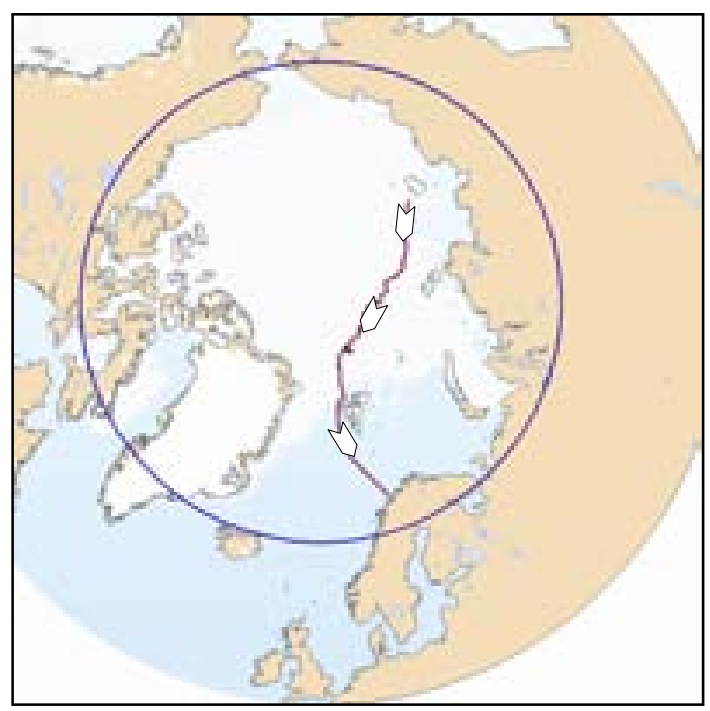

Abb. 2: Der Weg der „Fram“während der Drift 1893-1896.

Am Mittsommertag 1893 brach er mit zwölf Begleitern von Tromsø zur Insel Nowaja Semlja auf. Kapitän war Otto Neumann Sverdrup, der später selber Entdeckungsfahrten leitete. Im Polarmeer angekommen, musste er die „Fram“ der Eisdrift überlassen. Die Zeit wurde für konsequente Messungen der Meeres- und Eisverhältnisse genutzt. Als nach anderthalb Jahren deutlich wurde, dass die Drift weit südlich des Pols vorbeiführte und damit die Hoffnung zunichte machte, ihn auf diese Weise zu erreichen, versuchte Fridtjof Nansen mit seinem Begleiter 
Hjalmar Johansen am 14.03.1895 von der „Fram“ aus per Ski und Hundeschlitten den Pol zu erreichen. Er gelangte bis $86^{\circ} 14^{\prime}$ nördlicher Breite, so nah wie nie ein Mensch zuvor dem Pol gewesen war. Unter schwersten Strapazen schlugen sie sich nach Franz-Josef-Land durch, wo sie überwinterten. Am 17. Juni 1896 stieBen sie zufällig auf eine britische Expedition. Mit deren Hilfe gelangten sie nach Tromsø zurück. Eine Woche später traf dort auch die als verschollen geltende „Fram“ ein, die kurz zuvor nordwestlich von Spitzbergen vom Packeis freigegeben worden war.

Wenn auch das eigentliche Expeditionsziel nicht vollkommen erreicht wurde, so war die wissenschaftliche Ausbeute außerordentlich groß. Das Schiff, das später auch von Otto Neumann Sverdrup und Roald Amundsen für Polarfahrten genutzt wurde, liegt seit 1936 auf der Halbinsel Bygdøy in Oslo im „Fram Museum“.

Die These über die nicht gestörte Westdrift im Polarmeer konnte 2002 in einer Zusammenarbeit von Wissenschaftlern aus Göttingen und Kiel mit Hilfe von modernen isotopengeochemischen Analysen gelöst werden. Altersdatierungen an einem Sedimentkern aus dem Arktischen Meer und Sedimentproben der umrandenden Schelfgebiete zeigten nicht nur die Klimaschwankungen im Arktischen Ozean, sondern auch, dass die Meeresströmungen und somit die vom Treibeis verfrachteten Sedimente den von Nansen empirisch nachgewiesenen Weg der Eisdrift genommen hatten (Tütken et al. 2002).

Basierend auf Liefergebietsanalysen können Aussagen über globale Klimaschwankungen gemacht werden. In allen Klimamodellen ist die Oberflächen-

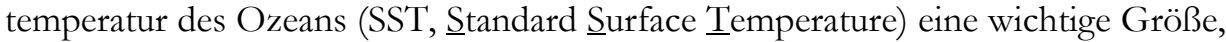
da diese die Bedingungen an der Grenzschicht „Ozean-Atmosphäre“ kontrolliert (Beck et al. 1992). Es besteht daher ein starkes Interesse, die SST der Vergangenheit, insbesondere der Tropen, zu rekonstruieren. Als SST-Thermometer dienen die sogenannten Proxys, das sind von der Temperatur abhängige Isotopen$\left(\delta^{18} \mathrm{O}\right)$ oder Element-Verhältnisse (Sr/Ca, U/Ca, Mg/Ca oder F/Ca) in kalzitischen und aragonitischen Foraminiferen und gebänderten aragonitischen Korallen. Die moderne Isotopenanalytik bietet die Möglichkeit, solche Elementverhältnisse sehr genau zu bestimmen. An einem Beispiel ist in Abb. 3 dargestellt, wie durch Sr/Ca- und U/Ca- Thermometrie an Korallen (Porites lutea) im Indischen Ozean vor der Westküste Australiens Temperaturschwankungen mit einer Genauigkeit von ca. $1^{\circ} \mathrm{C}$ bestimmt werden können.

Der Ausbruch des Pinatubo 1991 ist in den Korallen des Ningaloo Reefs durch einen SST-Abfall gegenüber dem Langzeitmittelwert um ca. $1^{\circ} \mathrm{C}$ im Jahr 1992 und 1,9 ${ }^{\circ} \mathrm{C}$ im Jahr 1993 aufgezeichnet. Durch den asiatischen Monsun verursachte SST-Anomalien scheinen sich ebenfalls an der westaustralischen Küste auszuwirken. 


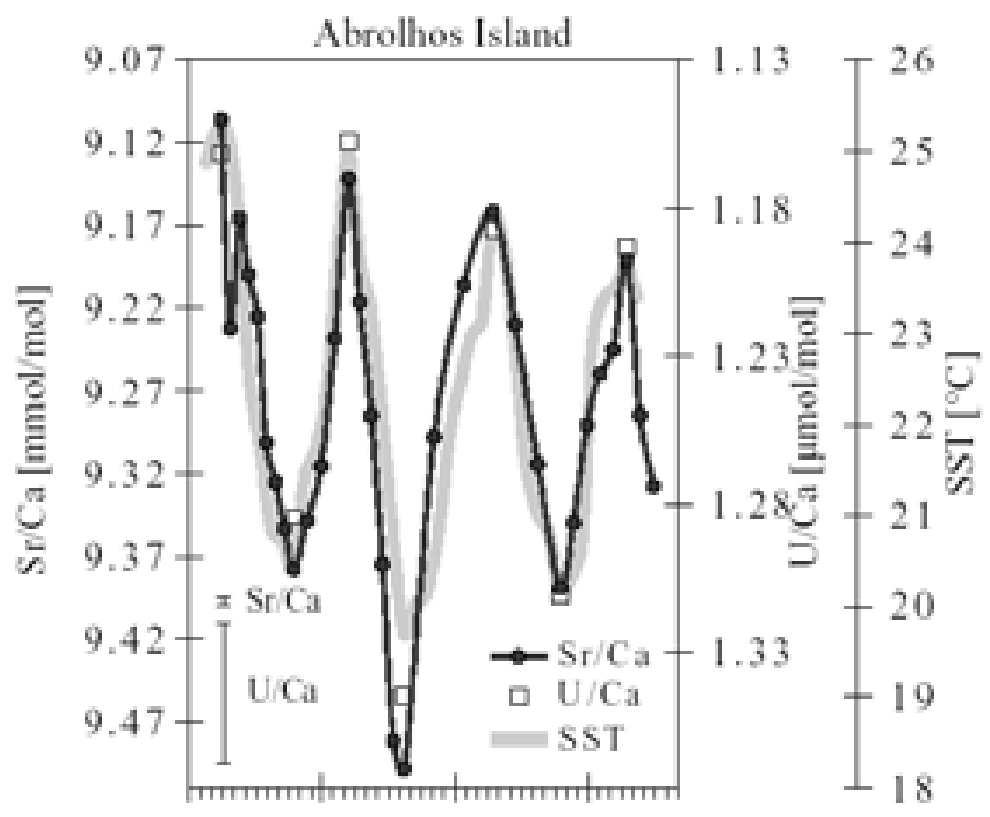

Jan 89 Jan 90 Jan 91 Jan 92

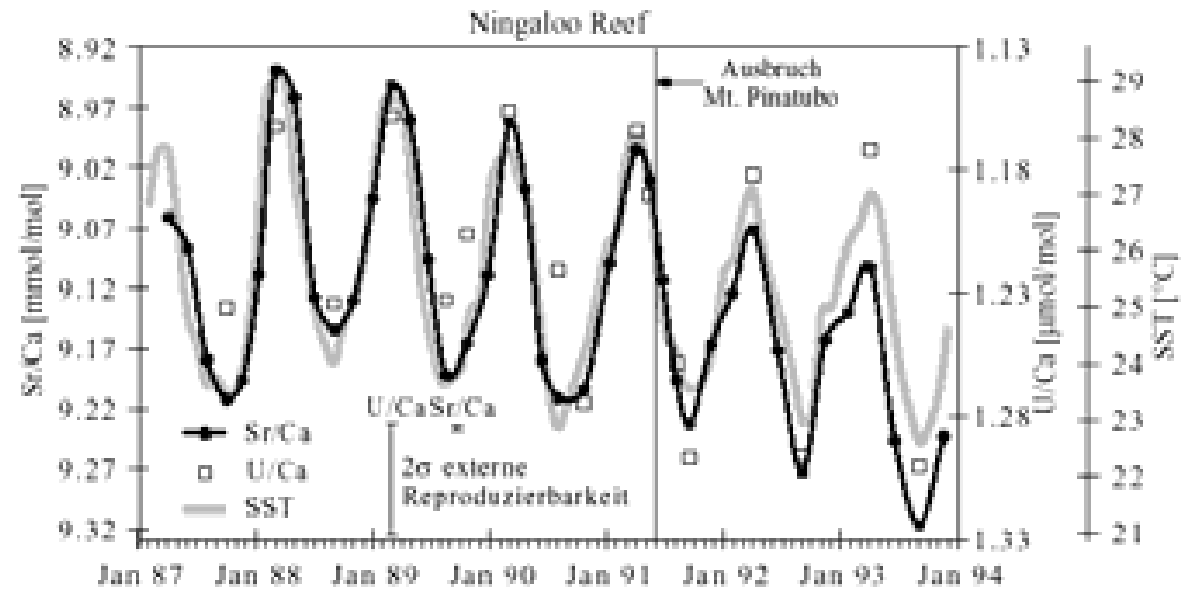

Abb. 3: Vergleich quischen SST-Daten, Sr/Ca- und U/Ca-Verbältnissen in Porites lutea, Lokalität Abrolhos Island und Ningaloo Reef (Wischow 1999). 
Damit die zukünftige klimatische Entwicklung in engen Grenzen vorausgesagt werden kann, ist die Gewinnung von SST-Daten mit hoher räumlicher und zeitlicher Auflösung von entscheidender Bedeutung, um damit die computergestützten Klimamodelle zu überprüfen und zu eichen.

Neben den Vorhersagen zur Klimaentwicklung wird in Zukunft vor allem auch die Verfügbarkeit des Wassers eine entscheidende Rolle spielen. Für diese Fragestellung können wir wiederum auf unsere Isotopensysteme zurückgreifen, in

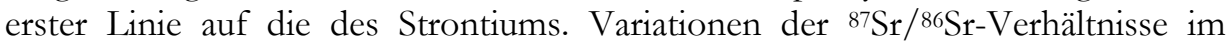
Grundwasser zeigen eine Abhängigkeit von verschiedenen Faktoren, unter denen die mineralogisch-geochemische Zusammensetzung des Aquifers bzw. des Einzugsgebietes dominieren. Die Sr-Isotopenverhältnisse in natürlichen Wässern werden über Wechselwirkungen zwischen Wasser und Mineralen kontrolliert. Für die Isotopensignatur des Wassers spielen unterschiedliche Löslichkeiten der verwitternden Minerale die entscheidende Rolle, aber auch anthropogene Quellen müssen berücksichtigt werden. Die Strontium-Isotopensystematik kann somit als „Tracer“ zur Berechnung von Mischungen zweier unterschiedlicher Wässer eingesetzt werden.

Zur Trinkwasserversorgung der Stadt Göttingen wird seit 1980 eine kontrollierte Mischung von Grundwässern der Eigenförderung mit Oberflächenwässern der Sösetalsperre (Harz) im Verhältnis von etwa 1:4 vorgenommen. Die Untersuchungen (Wiegand et al. 1998) zeigen, dass die beprobten Göttinger Wasserwerke Mischungsverhältnisse von $76,5 \%$ bis $86,4 \%$ aufweisen und diese mit den von den Wasserwerken angegebenen Werten übereinstimmen. Die Strontium-Isotopie erlaubt uns nicht nur, Mischungsverhältnisse zu berechnen, sondern gibt aus den oben genannten Gründen auch Aufschluss über die Aquifere und somit schließlich über die Fliesswege des Wassers. Diese Technik wurde im Rahmen einer Zusammenarbeit der Arbeitsgruppe Isotopengeologie am GZG und der Harzwasserwerke $\mathrm{GmbH}$ eingesetzt, um ein Modell der Grundwasserfließwege im Aquifer von Liebenau II (NW Deutschland) zu entwickeln.

Basierend auf den Unterschieden der Sr-Isotopensignaturen in den Grundwässern konnten für das Einzugsgebiet des Wasserwerkes Liebenau II vier Grundwasserhorizonte in den quartären Lockersedimenten unterschieden werden (Abb. 4). Der Vergleich zwischen Sr-Isotopenverteilung und Zusammensetzung der Hauptkomponenten der Grundwässer zeigt gute Übereinstimmungen für die unterschiedlichen Grundwasserhorizonte und bestätigt, dass dieselben Prozesse für die chemischen und isotopenchemischen Variationen der Grundwässer verantwortlich sind. Über charakteristische Unterschiede in den ${ }^{87} \mathrm{Sr} /{ }^{86} \mathrm{Sr}-V$ erhältnissen konnte zwischen Silikat- und Karbonatauflösung unterschieden werden (Wiegend et al. 2001). 


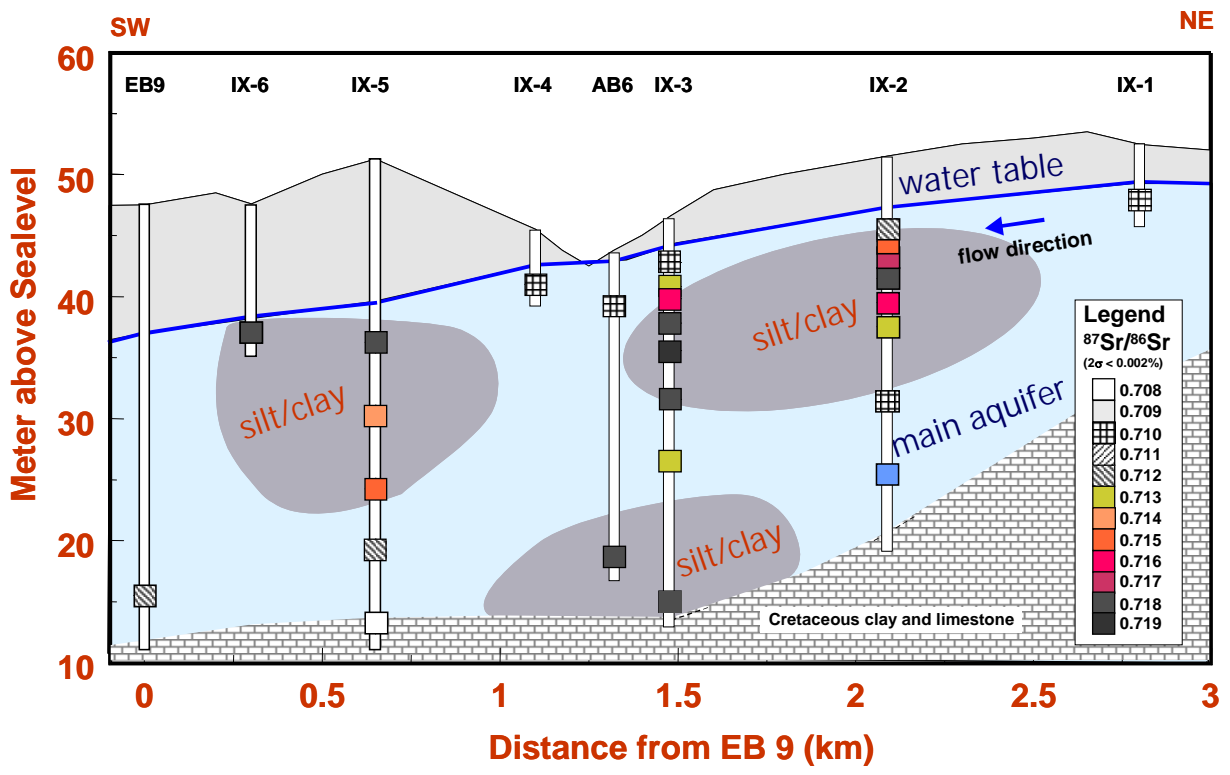

Abb. 4: Modell der Grundwasserfließwege im Aquifer von Liebenau II, dargestellt anhand der Brunnengalerie 9 (nach Wiegand et al. 2001).

Der Großraum Bangkok leidet wie viele Ballungsgebiete unter Landabsenkung wegen des enorm hohen Verbrauchs an Grundwasser. Die Entnahme von bis zu 1,4 Millionen Kubikmeter pro Tag führt zu Landabsenkungsraten bis zur 10 Zentimeter pro Jahr (Ramnarong \& Buapeng 1992). Diese Absenkungen haben zu nicht unerheblichen Schäden an der Infrastruktur dieser Region beigetragen. Eine mögliche Maßnahme zur Eindämmung dieser Absenkung wäre die Reinjektion von gereinigtem Oberflächenwasser. Damit die Fließwege des reinjizierten Wassers verfolgt werden können, müssen Parameter analysiert werden, die ohne künstliche chemische Zusätze bestimmt werden können. Basierend auf Erkenntnissen der Studie für das Wasserwerk Liebenau II, wurden in einer Langzeitstudie mehrere Entnahmebrunnen aus drei unterschiedlichen Aquiferen im Großraum Bangkok auf ihre Strontium-Isotopie untersucht, um die Konstanz der Zusammensetzung zu überprüfen. Diese Untersuchungen haben gezeigt, dass ausreichende Unterschiede in den ${ }^{87} \mathrm{Sr} /{ }^{86} \mathrm{Sr}-\mathrm{Verhältnissen} \mathrm{zwischen} \mathrm{Grund-} \mathrm{und} \mathrm{Ober-}$ flächenwasser vorhanden sind und dass diese über Jahre konstant sind (Hansen et al. 2002). Damit wäre ein Monitoring des reinjizierten Wassers möglich. Sollte aber die Entscheidung zugunsten der Versorgung ausschließlich mit Oberflächenwasser erfolgen, könnte jederzeit eine illegale Wasserentnahme aus den Aquiferen nachgewiesen werden. 
Nach diesem Exkurs durch einige der Anwendungen der modernen Isotopenanalytik möchte ich zu der Anfangs gestellten Frage nach dem Alter der Erde zurückkehren. Die bis heute beste Bestimmung für das Alter der Formation der

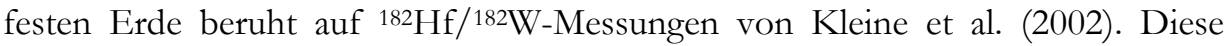
Autoren geben das Alter der Erde mit 4,53 \pm 0,002 Milliarden Jahre an, für die Formation unseres Sonnensystems wird ein Alter von 5,566 \pm 0,002 Milliarden Jahren errechnet. Auch wenn diese Bestimmungen nahe an dem eigentlichen Erdalter liegen, wird die Suche nach noch genaueren Datierungen weitergehen. Eine Suche, die Arthur Holmes (1913) in folgendem Satz formulierte:

\section{„It is perhaps a little indelicate to ask our Mother Earth her age, but Science acknowledges no Shame and from time to time has boldly attempted to wrest from her a secret which is proverbially well guarded."}

\section{Literatur}

Ahrendt, H., Hansen, B. T., Lumjuan, A., Mickein, A. and Wemmer, K. (1997):

Tectonometamorphic evolution of NW-Thailand deduced from U/Pb-, $\mathrm{Rb} / \mathrm{Sr}-, \mathrm{Sm} / \mathrm{Nd}$ - and K/Ar-isotope investigations. In: IGCP 359 and IGCP 383 Proc. Of the Internat. Conference on Stratigraphy and Tectonic Evolution of Southeast Asia and the South Pacific, 19-24 August 1997, Bangkok, 314-319

Aldrich, L. T. and Nier, A. O. (1948): Argon 40 in potassium minerals, Phys. Rev., $74,876-877$

Andrasko, J., Kopp, I., Åbrink, Å. and Skjöld, T. (1993): Lead Isotope Ratios in Lead Smears and Bullet Fragments and Application in Firearm Investigations, Journal of Forensic Sciences, JFSCA, Vol. 38, No 5, 1161-1171

Beck, J. W., Edwards, R. L., Ito, E., Taylor, F. W., Recy, J., Rougerie, F., Joannot, P. and Henin, C. (1992): Sea-surface temperature from coral skeletal strontium/calcium ratios, Science 257, 644-647

Becquerel, H. (1896) : Sur les radiations invisibles émises par phosphorescence ; Sur les radiations invisibles émises par les corps phosphorescents; Sur les radiations invisibles émises par les sels d'úranium, Compt. Rend., 122, 420, 501,689

Black, L. P., Gale, N. H., Moorbath, S., Pankhurst, R. J. and McGregor, V. R. (1971): Isotope dating of very early Precambrian amphibolite facies gneisses from the Godthaab District, West Greenland, Earth Planet. Sci. Lett. 12, 245-259 
Burnet, T. (1681): Telluris theoria sacra: Orbis nostril originem \& mutationes generales, quas aut jam subiit, aut olim subiturus est, complectens. Libri duo priores de diluvio \& paradiso, London: Typis R. N., Impensis G. Kettilby, 306 p.

Eisenhauer, A., Wischow, D., Heiss, G., Dullo, W.-C. and Hansen, B. (1999): $\mathrm{Sr} / \mathrm{Ca}$ and U/Ca Thermometry on Porites Lutea Corals from the Indian Ocean, Journal of Conference Abstracts 4/1, Cambridge Publications, 198

Glimscher, M. J. (1976): Composition, structure, and organisation of bone and other mineralised tissues and the mechanism of calcification. In: Handbook of physiology - Endocrinology, Chapter 7, Williams and Wilkins, Baltimore

Grupe, G., Price, T. D., Schröter, P., Söllner, F., Johnson, C. and Beard, B. L. (1997): Mobility of Bell Beaker people revealed by Strontium isotope ratios of tooth and bone: A study of southern Bavarian skeletal remains, Applied Geochemistry 12, 517-525

Hansen, B. T., Pawlig, S., Chusanathas, S. and Buapeng., S. (2002): ${ }^{87} \mathrm{Sr} /{ }^{86} \mathrm{Sr}-$ Ratios: A natural Tracer to Characterize Aquifers and Groundwater Flow Paths: Examples from the Area, NE of Bangkok, The Symposium on Geology of Thailand 26-31 August 2002, Bangkok, Thailand

Holmes, A. (1913): The age of the Earth, Harper and Brothers, London, 194 p.

Horn, P., Hölzl, S., Todt, W. and Matthies, D. (1998): Isotope abundance Ratios of Sr in Wine Provenance Determinations, in a Tree-Root activity Study, and of $\mathrm{Pb}$ in a Pollution Study on Tree-Rings, Isotopes Environ. Health Stud. 34, 31-42

Hutton, J. (1785): Theory of the Earth, Transactions of the Royal Society of Edinburgh, 1788, Vol. I

Johansson, Á. (1992): En Blyisotopstudie av Karl XIIs kulknapp, Varbergs Museum-Årsbok, 15-23

Kleine. T., Münker, C., Mezger, K. and Palme H. (2002): Rapid accretion and early core formation on asteroids and the terrestrial planets from Hf-W chronometry, Nature 418, 952-955

Lyell, C. (1830): Principles of Geology, Vol. I-III, John Murray, London, 1830-1833

Messer Griesheim (2002): Isotope überführen Briefbombenleger, Gas Aktuell 24,1

Mendelejew, D. (1869): Ueber die Beziehungen der Eigenschaften zu den Atomgewichten der Elemente, Zeitschrift für Chemie 12, 405-406 
Mojzsis, S. J. and Harrison (2002): Establishment of a 3.83-Ga magmatic age for the Akilia tonalite (southern West Greenland), Earth. Planet. Sci. Lett. 202, 563-576

Nier, A. O. (1940): A mass spectrometer for routine isotope abundance measurements, Rev. Sci. Instrum., 11, 212-216

Ramnarong, V. \& Buapeng, S: (1992): Groundwater Resources of Bangkok and its Vicinity: Impact and Management. In: Proc. National Conf. Geol. Res. Thailand: Potential for future Development, Dept. Min. Res., Bangkok, 172-184

Richards, T. W., and Lembert, M. E. (1914): Atomic weight of lead of radioactive origin, J. Am. Chem. Soc., 36, 1329-1344

Rossmann, A., Haberhauer, G., Hölzl, S., Horn, P., Pichlmayer, F. and Voerkelius, S. (2000): The potential of multielement stable analyses for regional origin assignment of butter, Eur. Food Technol. 21, 32-40

Rutherford, E. and Soddy, F. (1902a): The cause and nature of radioactivity, Pt. I. Phil. Mag., ser. 6, 4, 370-396

Rutherford, E. and Soddy, F. (1902b): The cause and nature of radioactivity, Pt. II. Phil. Mag., ser. 6, 4, 569-585

Schroeder, H., Tipton, I. and Nason, I. (1972): Trace metals in man: Strontium and barium, Journal of Chronic Diseases 25, 491-517

Schutkowski, H., Hansen, B., Wormuth, M. and Herrmann, B. (2001): Signaturen Stabiler Strontium-Isotope in menschlichen Hartgeweben- Möglichkeiten für die osteologische Identifikation. In: Oehmichen, M. Geserick, G. (Hrsg.): Osteologische Identifikation und Altersschätzung, Research in Legal Medicine, 26, Schmidt-Römhild, Lübeck, 31-40

Steno, N. (1669): De solido intra solidum naturaliter contento dissertationis prodromus. In: White, G. W. (Ed.): Contributions to the History of Geology, Hafner, New York, Vol. 4, 1968

Tütken, T., Eisenhauer, A., Wiegand, B. and Hansen, B. T. (2002): Glacialinterglacial cycles in $\mathrm{Sr}$ and $\mathrm{Nd}$ isotopic composition of Artic marine sediments triggered by the Svalbard/Barents Sea ice sheet, Marine Geology, $182,351-372$

Wedepohl, K. H., Delevaux, M. H. and Doe, B. R. (1978): The potential source of lead in the Permian Kupferschiefer bed of Europe and some selected Paleozoic mineral deposits in the Federal Republic of Germany, Contrib. to Min. and Petr. 65, 273-271 
Wemmer, K., Sievers, H., Ta Trong Thzang; Phan Trong Trinh (1999): New hints for nappe tectonics in Nothern Viet Nam by K/Ar dating of very low grade sediments, Journal of Geology, Series B 13-14, 107-109

Wiegand, B., Dietzel, M., Bielert, U., Groth, P. and Hansen, B. T. (2001): ${ }^{87} \mathrm{Sr} / 86 \mathrm{Sr}-$ Verhältnisse als Tracer für geochemische Prozesse in einem Lockergesteinsaquifer (Libenau, NW-Deutschland), Acta hydrochim. Hydrobiol. 29, 2-3, 139-152

Wischow, D. (1999): Sr/Ca- und U/Ca-Thermometrie an Korallen (Porites lutea) aus dem Indischen Ozean, Diss. Univer. Göttingen. 78 p.

Wormuth, M. Hansen, B. T. and Schutkowski, H. (2000): ${ }^{87} \mathrm{Sr} /{ }^{86} \mathrm{Sr}-$ Verhältnisse in menschlichem Knochen und Zahnschmelz als Indikator für Ortswechsel zu Lebzeiten, Beihefte zum Eur. Jour. of Mineralogy, 12, 238 



\title{
Fernerkundung der Erde - Neue Sensoren und innovative Techniken
}

\author{
Martin Kappas
}

\section{Einleitung}

Elias Canetti sagte einmal: „Denn ein Weg zur Wirklichkeit geht über Bilder“, und ein chinesisches Sprichwort lautet: „Ein Bild sagt mehr als 1000 Worte“. Diese Zitate sollen in den Themenkomplex einführen, wie wir unsere Erde sehen, wahrnehmen und Zugang zur Wirklichkeit der Erdphänomene erlangen. Wenn wir unsere Sensoren, die Augen, öffnen, sehen wir etwas - und wir glauben, es sei die Wirklichkeit oder Realität. Der Vorgang des Sehens und Erkennens ist für uns so selbstverständlich und vertraut, dass wir normalerweise gar nicht auf die Idee kommen, an ihm zu zweifeln. Das Sehen vermittelt uns weitaus das meiste, was wir von der Erde wissen, es hilft uns, uns auf ihr zurecht zu finden und uns zu bewegen, auf ihr zu handeln und sie zu gestalten. Aber so selbstverständlich uns dies alles ist, so wenig wissen wir darüber, wie sich dieses Sehen eigentlich abspielt: Die Vertrautheit des Vorgangs verbirgt seine Problematik. Dieser Prozess der Wahrnehmung mit unseren ureigenen Sensoren, den Augen, lässt sich auf die moderne Fernerkundung der Erde übertragen. Vom physikalischen Aspekt aus gesehen gibt es eine Reizquelle, denn es basiert alles darauf, dass von Objekten, die wir oder Weltraumsensoren betrachten, elektromagnetische Strahlung ausgeht. In der Regel reflektieren die Objekte in unserer Umgebung einen Teil der auftref- 
fenden elektromagnetischen Strahlung und werden dadurch sichtbar. Die Mehrzahl der Objekte, die wir sehen, werden beleuchtet. Sie haben die Eigenschaft, einen Teil der elektromagnetischen Strahlung, die ankommt, zu reflektieren. Diese Reflektion hat unterschiedliche Intensität, deshalb erscheinen uns die Gegenstände auf der Erde unterschiedlich hell. Die Reflektion ist aber auch ein selektiver Prozess bezüglich der Wellenlängen; nur deshalb können wir die unterschiedlichen Objekte in verschiedenen Farben sehen. Visuelle Wahrnehmung setzt aber eine Strukturierung der aufgenommen Reize voraus. Die Welt, die wir um uns herum wahrnehmen, und ein Großteil der Flächen, die wir betrachten, weist eine gewisse Strukturierung auf, eine Oberflächenstruktur, die in diesem Zusammenhang Textur genannt wird. Es gibt auf Oberflächen oder Bildwiedergaben dieser Oberflächen gewisse Elemente, die einzeln eigentlich nichts aussagen. Aber in ihrer Gesamtheit ergeben sie eine Strukturierung der Fläche, die für einen bestimmten Oberflächentyp, für ein bestimmtes Material, für einen Vegetationsbestand im Luft- oder Satellitenbild typisch sind. In unserer Wahrnehmung spielen solche Texturen eine große Rolle, zumal sie in hohem Maße objektspezifisch sind.

Dieser Beitrag erläutert den derzeitigen Stand und die Zukunftsperspektiven der Fernerkundung der Erde. Aktuelle Trends in der Bilderfassung sowie der Bildauswertung werden aufgezeigt. Fernerkundung wird heute in unterschiedlichen Maßstabsbereichen und für unterschiedliche Zwecke von der Rasterelektronenmikroskopie bis zur Erkundung ganzer Planeten (Mars-Sonde) eingesetzt. Der Beitrag schließt mit einem Ausblick auf zukünftige Entwicklungen.

Die Fernerkundung bildet heute einen zentralen Inhalt einer modernen Geoinformatik. Die Entwicklung neuer Technologien sowie der Einsatz operationeller Positionserfassung via GPS (Global Position System), multisensoraler Satellitensysteme, Neuerungen in der digitalen Photogrammetrie und Laser Scanning erzeugen Daten der Erde mit immer höherer spektraler und räumlicher Genauigkeit. Hinzu kommt noch die temporale Betrachtung von Zeitreihen der unterschiedlichen Sensordaten (Monitoring). Die Verschmelzung moderner Bildverarbeitungsmethoden und die Integration der aus ihnen gewonnen Informationen in geographische Informationssysteme erzeugt ein effizientes Management dieser Daten und die Möglichkeit eines vielseitigen, interdisziplinären Datenzugriffs. Durch dieses Vorgehen sind unterschiedliche Geodaten seit kurzem in ihrer Gesamtheit (integrierte Speicherung und Zugriff) für fachübergreifende Fragestellungen nutzbar. Ein enormes Potenzial für die ganzheitliche Erforschung der Erde. Die Fernerkundung spielt hier die Schlüsselrolle in der Datenbereitstellung, wobei nicht nur die terrestrischen Bereiche der Erde, sondern auch Ozeane und die Atmosphäre erfasst werden. Die Fernerkundung stellt oftmals die einzige Möglichkeit dar, Daten von weit entlegenen oder schwer zugänglichen Regionen (Regenwälder Amazoniens; Eisflächen Antarktika, Grönland) zu erhalten, und ist die einzige Möglichkeit, flächenhaft Information über den Zustand unserer Erde abzuleiten. Ein wichtiger limitierender Faktor für den Einsatz von Fernerkundungsdaten sind zurzeit noch die Kosten für die neusten und hochauflösenden Sensoren. Die teilweise sehr hohen Kosten für die Sensordaten kommerzieller 
Betreiber (zurzeit Digital Globe und Space Imaging) stellen allerdings für viele Interessenten eine große Hürde da. Ein weiteres Problem in der Nutzung von Satellitendaten liegt aber immer noch darin, dass die professionelle Nutzung der Daten noch immer „Spezialisten“ obliegt. Die Daten liegen überwiegend als „Rohdaten“ vor oder als weiter entwickelte Fernerkundungsprodukte (z. B. strahlungskorrigierte Daten, geo-referenzierte Daten, IHS-transformierte Daten). Meist können diese veredelten Fernerkundungsprodukte aufgrund mangelndem Budget nicht gekauft werden. Die Rohdaten bedürfen aber einer aufwendigen Bearbeitungskette, bevor sie themenbezogen genutzt werden können. Dies führt dazu, dass die Mehrzahl der archivierten Fernerkundungsdaten in der Vergangenheit ungenutzt blieben bzw. in der Zukunft ungenutzt bleiben. Eine unglaubliche Verschwendung von Wissensressourcen. Dies zeigt aber auch gleichzeitig, dass die Ausbildung im Bereich „Fernerkundung - Geodatenverarbeitung“ in den einzelnen Fachwissenschaften in der Zukunft zur Grundausbildung gehören muss. Hiervon ist der Wissensstandort Deutschland aber noch weit entfernt. Dies belegen auch die Curricula der Geographiestandorte deutscher Universitäten sowie die Anzahl vorhandener Professuren im Bereich Fernerkundung/Geoinformatik. Eine intelligente Nutzung der neuen Geodaten (insb. der Satellitendaten) ist nur möglich, wenn diese Methoden in die einzelnen Fachwissenschaften integriert werden. Eine nachhaltige Nutzung von Satellitendaten bedarf also eines Umdenkens in der Bereitstellung der Daten, aber auch eines Umdenkens in den Curricula an deutschen Universitäten.

\section{Fernerkundungssysteme - ein Überblick}

Ein Fernerkundungssystem ist ein Sammelbegriff für eine große Anzahl von Möglichkeiten, kontaktlose Messungen an Objekten zu betreiben. Wie kann man nun Fernerkundung definieren? „Fernerkundung ist zu einer selbständigen Wissenschaft herangewachsen, welche durch kontaktlose Messung Informationen über Objekte zu erhalten versucht“" (Kappas 1994). Dabei setzt sich die Fernerkundung aus zwei Haupttätigkeiten zusammen:

1. Datenerfassung

2. Datenanalyse und Interpretation.

Die moderne Fernerkundung hat sich dabei in den letzten Jahren weit von der klassischen Luftbildfernerkundung und Photogrammetrie entfernt bzw. weiter entwickelt. Viele der traditionell als photogrammetrisch bezeichneten Aufgaben werden heute weitgehend automatisch oder mit Hilfe alternativer Techniken gelöst. Heute werden neben dem sichtbaren Licht weitere elektromagnetische Wellenlängen genutzt, um Informationen über Objekte abzuleiten. Hierzu gehören insbesondere Wellenlängen im nahen und mittleren Infrarot, im thermalen Infrarot und im Mikrowellenbereich (Radar, vgl. Abb. 1). 


\section{Das elektromagnetische Spektrum}

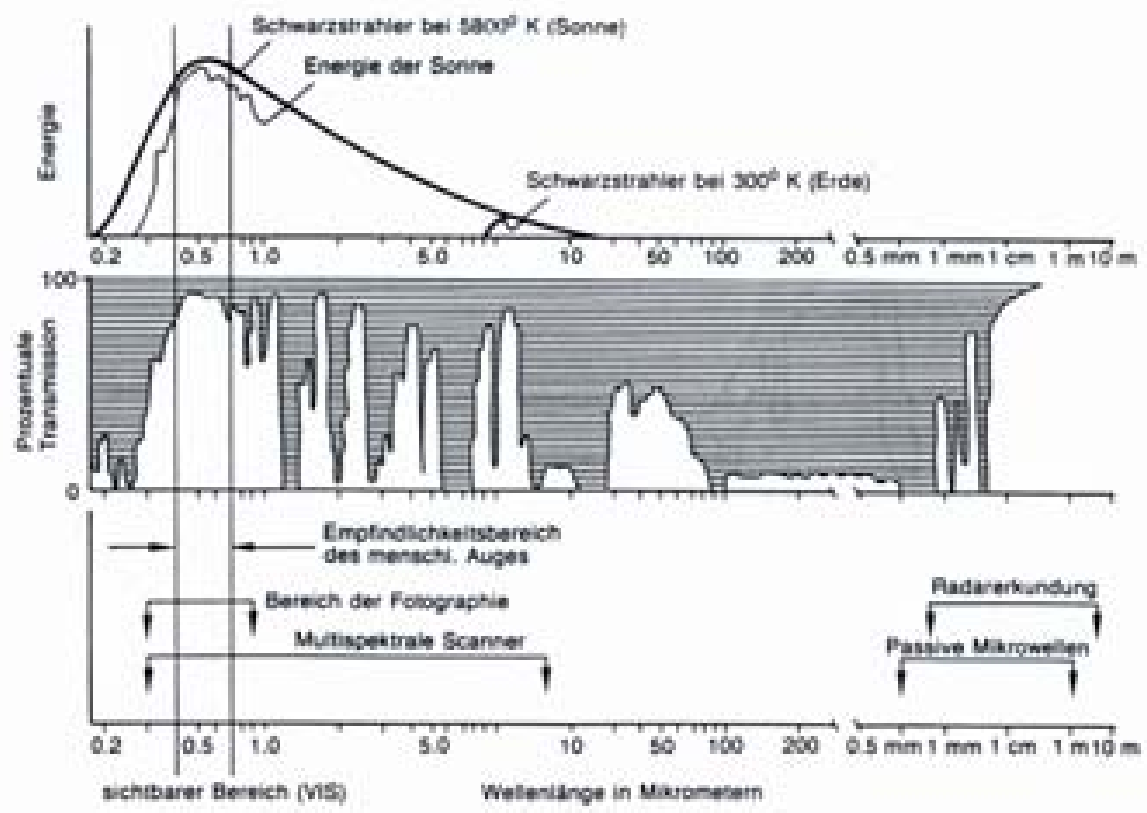

Abb. 1: Das elektromagnetische Spektrum - Kontinuum und Informationsträger (Kappas 1994, S. 7).

Letzteres teilt die Fernerkundungssysteme in aktive (Radar, Lidar) und passive Systeme. Passive Systeme empfangen lediglich die reflektierte oder emittierte Strahlung von der Erdoberfläche. Aktive Systeme fungieren als eigene Beleuchtungsquelle und sind somit von Tages- und Nachtzeit sowie von der Bewölkung unabhängig. Eine weitere Unterscheidung ergibt sich durch die Wahl der Aufnahmeplattform. Hier unterscheidet man zwischen satellitengetragenen und flugzeuggetragenen Systemen. In neuster Zeit werden aber auch Drohnen (ferngelenkte Flugkörper), Ballons oder Hebebühnen (z. B. in der terrestrischen Hyperspektralfernerkundung) eingesetzt. Weitere Einteilungskriterien von Fernerkundungssystemen sind die spektrale, räumliche und radiometrische Auflösung. Moderne Fernerkundungssysteme sind heute zumeist satellitengetragen und zeichnen ihre Daten direkt digital auf. Die Tabelle 1 fasst die Einteilung von Fernerkundungssystemen systematisch zusammen. 
Fernerkundung der Erde

\begin{tabular}{|c|c|c|c|c|c|c|c|c|}
\hline \multicolumn{9}{|c|}{ Taxonomie der Fernerkundungssysteme } \\
\hline $\begin{array}{l}\text { Auftreichenende } \\
\text { Plattlorm }\end{array}$ & \multicolumn{3}{|c|}{ Satelia/Space Shuate } & \multicolumn{2}{|c|}{ Flugeveg / Ballon / Drohne } & \multicolumn{3}{|c|}{ Stationarer Sensor } \\
\hline $\begin{array}{l}\text { Autreichnunger- } \\
\text { modus }\end{array}$ & \multicolumn{4}{|c|}{ patsiv / elektrocplisch } & \multicolumn{4}{|c|}{ abdv (Laser, Radar) } \\
\hline $\begin{array}{l}\text { Aufreichnungs } \\
\text { medium }\end{array}$ & \multicolumn{4}{|c|}{ andog (Kamera, Vides) } & \multicolumn{4}{|c|}{ Dighal (Whiskereom, Line Array, OCD) } \\
\hline $\begin{array}{l}\text { Spektrale } \\
\text { Abdeckung }\end{array}$ & \multicolumn{2}{|c|}{$\begin{array}{l}\text { Sichoat/ } \\
\text { Ultraviolet }\end{array}$} & \multicolumn{2}{|c|}{ Reflekterses intrard } & \multicolumn{2}{|c|}{ Thermales Intrarct } & \multicolumn{2}{|c|}{ Microwelen } \\
\hline $\begin{array}{l}\text { Spektrale } \\
\text { Aunosuing }\end{array}$ & \multicolumn{2}{|c|}{$\begin{array}{l}\text { Panchromatseh } \\
\text { (1 Band? }\end{array}$} & \multicolumn{2}{|c|}{$\begin{array}{l}\text { Mulsoebtral } \\
\text { (2-20 Blnder) }\end{array}$} & \multicolumn{2}{|c|}{$\begin{array}{l}\text { Hypersceitral } \\
\text { (20-250 Bander) }\end{array}$} & \multicolumn{2}{|c|}{$\begin{array}{l}\text { Utrasceitral } \\
\text { is } 250 \text { Blnden? }\end{array}$} \\
\hline $\begin{array}{l}\text { Radiometrische } \\
\text { Aufiosung }\end{array}$ & \multicolumn{2}{|c|}{ Sehe hoch \& $>12$ bC) } & \multicolumn{2}{|c|}{ Hoch $(8-12$ bit $)$} & \multicolumn{2}{|c|}{$\operatorname{Mettel}(6-8 \mathrm{bC})$} & \multicolumn{2}{|c|}{ niedrig $(<6$ bel) } \\
\hline $\begin{array}{l}\text { Raumliche } \\
\text { Auflosung }\end{array}$ & $\begin{array}{l}\text { Hochst. } \\
\text { aufiosend } \\
\text { (< } \mathrm{t} \text { m) }\end{array}$ & Sehn & $\begin{array}{l}\text { Hoch } \\
\text { m) }\end{array}$ & $\begin{array}{l}\text { Hooh } \\
(4-10 \mathrm{~m})\end{array}$ & $\begin{array}{c}\text { Moted } \\
(10-50=)\end{array}$ & $\mathrm{Ne}_{150-1}$ & & $\begin{array}{l}\text { Sehr nocrio } \\
(>250 \mathrm{~m})\end{array}$ \\
\hline
\end{tabular}

Tab. 1: Taxonomie der Fernerkundungssysteme.

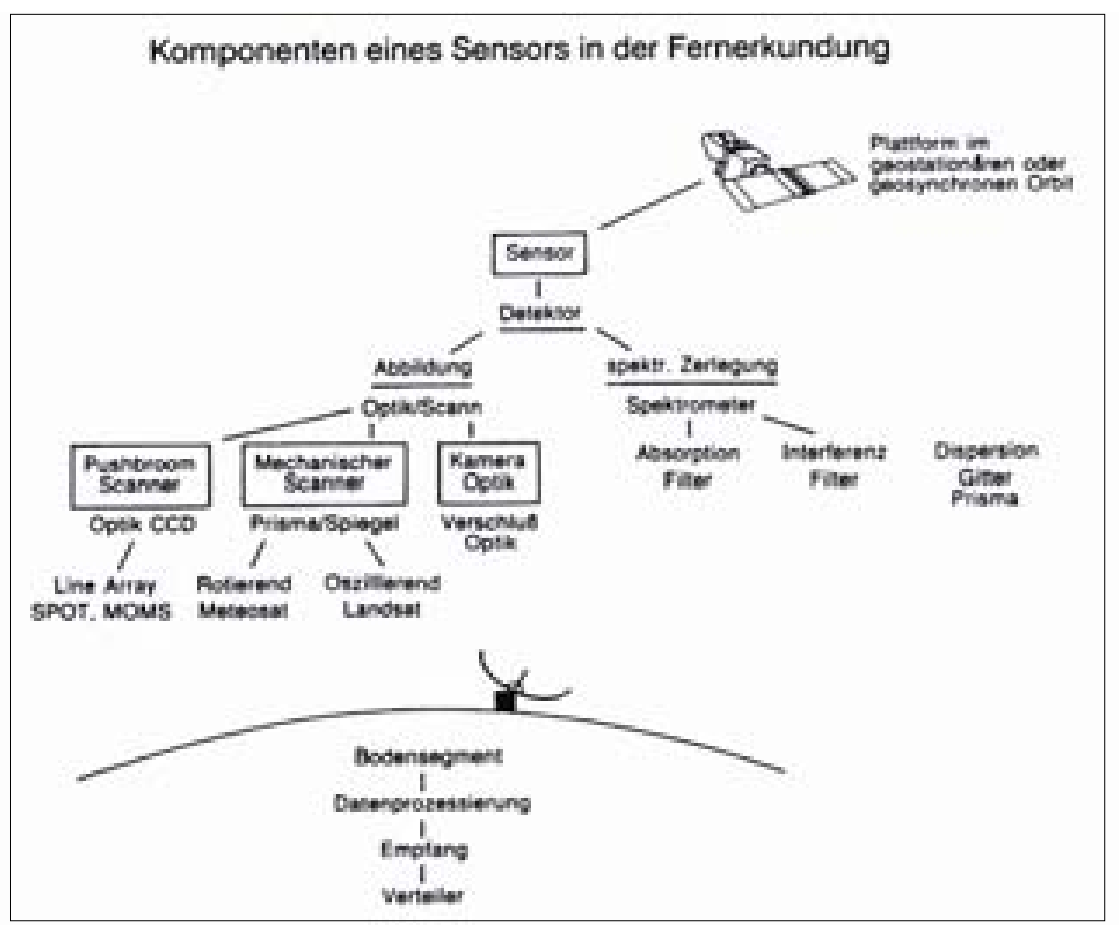

Abb. 2: Komponenten eines Sensors in der Fernerkundung (Kappas, 1994, S. 17). 


\section{Fernerkundungssysteme - state of the art}

\subsection{Mittlere bis hochauflösende Satellitensysteme}

In diesem Beitrag wird ein Schwerpunkt auf die optischen Systeme gelegt. Radarsysteme, mit ihrem unbestrittenen Nutzen für Militär oder Ozeanforschung, werden unter 3.3 kurz behandelt. Von den satellitengestützten operationellen Systemen sind insbesondere die Multispektralsysteme mittlerer und hoher Auflösung zu nennen. Diese Multispektralscanner liefern den Hauptanteil der operationellen Fernerkundungsdaten und sind auch für multitemporale Anwendungen zu nutzen (Monitoring). So reicht die Aufzeichnung der amerikanischen LandsatFamilie bis in das Jahr 1982 zurück, und der letzte Sproß der Landsat-Familie, der Landsat Enhanced Thematic Mapper (ETM) bietet im panchromatischen Bereich eine Raumauflösung von $15 \mathrm{~m}$. Als weitere operationell arbeitende Systeme in diesem Segment sind das französische SPOT-System und das indische IRS-System mit 5,8 m Auflösung (IRS 1C/1D) im panchromatischen Bereich zu nennen. Mit ihrer meist höheren Szenengröße (185 x $185 \mathrm{~km}$ bei Landsat TM) ergibt sich mittlerweile ein attraktives Preis/Leistungsverhältnis. Zudem sind diese Daten durch den integrierenden Charakter ihrer Pixelauflösung mit Standard-Bildverarbeitungsverfahren auswertbar. Die Tabelle 2 zeigt die Parameter der drei wichtigsten operationellen Systeme im mittel- bis hochauflösendem Bereich.

\begin{tabular}{|c|c|c|c|}
\hline Insticution & NASA (USA) & Ches Orankreich) & Neltsa (ledient \\
\hline Sabelibenursten & Landeat 7 ETU & SPOT 34 HAV & IRS ICO \\
\hline Mufaeichnugemodos & pan / MS + fhernal & Den IMS & $\operatorname{pan} /$ LISS $\mathrm{n}$ \\
\hline Abumliche Autlosung & $15 \mathrm{~m} / 39 \mathrm{~m}, 06 \mathrm{~m}$ ind & $90 \mathrm{n} / 20 \mathrm{~m}$ & $5.8 m / 23.5 m$ \\
\hline \multirow[t]{7}{*}{ Spebtrale Aunosung [nm] } & $500-900 / 450-520$ (b) & $510-699 / 580-590(9)$ & $500-750 / 520-500$ isl \\
\hline & $500-610[0)$ & $610-6998$ & 6000000 \\
\hline & $600-0908$ & $290-690(\mathrm{nin})$ & 7ro-ved inir) \\
\hline & 700-910 (nin) & $1500-1750$ (nar) & $1550-1700$ (mir) \\
\hline & 1570-1700 Amin) & & \\
\hline & $10420-12500$ (fr) & & \\
\hline & $2000-2350$ (min) & & \\
\hline Sinenenabdeckung & Ias $\times 1$ las km & $60 \times 60 \mathrm{~km}$ & $23 \times 23$ coker $70 \times 70 \mathrm{~km}$ \\
\hline Orbianche & $604 \mathrm{~km}$ & ase kn & 617 km \\
\hline inklination & $2 a 2^{+}$(tionnentynction) & Sa, $7^{*}$ (sornentynchion) & 9a., $1^{+}$(somnerdiychiron) \\
\hline Roweendungsmabstab & $1: 100000$ & $1=50000$ & $1: 25000$ \\
\hline
\end{tabular}

Tab. 2: Mittlere bis hochauflösende Satellitensysteme.

\subsection{Höchstauflösende Satellitensysteme}

Die Gruppe dieser Systeme wird seit 2000 von IKONOS II mit einer Auflösung von $1 \mathrm{~m}$ (panchromtisch) und $4 \mathrm{~m}$ (multispektral) und seit 2002 von QUICKBIRD mit einer räumlichen Auflösung von $0,6 \mathrm{~m}$ (panchromatisch) und 3-4 m (multispektral) angeführt. Weitere Entwicklungen mit höherer Auflösung werden 
folgen. Diese beiden kommerziellen Satellitensysteme reichen als Erste in den Anwendungsbereich der Luftbildphotographie hinein und ermöglichen Anwendungen in der kommunalen Planung. Durch schwenkbare Aufnahmesysteme konnte die Repetitionsrate deutlich gesenkt werden, sodass jeder Punkt der Erde in zwei bis drei Tagen aufgezeichnet werden kann. Neben diesen räumlich-höchstauflösenden Systemen sind spektral hochauflösende Systeme zu nennen (Hyperspektralsensoren), die allerdings zurzeit noch ausschließlich von Flugzeugen getragen werden. Aber erste hyperspektrale Sensoren für den Weltraum sind in Vorbereitung bzw. befinden sich bereits auf Plattformen. Der europäische ENVISATSatellit verfügt über eine Vielzahl von Sensoren (Multisensorik). Ebenso besitzt die TERRA-Satellitenfamilie der NASA eine Vielzahl von Sensoren, von denen ein Sensor (MODIS, 36 Kanäle) erstmals hyperspektrale Eigenschaften aufweist.

\begin{tabular}{|c|c|c|}
\hline Institution & Digital Globe & Space Imaging \\
\hline Satellitensystem & QuickBird & konos \\
\hline Aufzeichnugsmedus & pan / MS + thermal & pan / MS \\
\hline Räumliche Auflósung & $0.61 \mathrm{~m} / 2.50 \mathrm{~m}$ & $1 \mathrm{~m} / 4 \mathrm{~m}$ \\
\hline \multirow[t]{4}{*}{ Spektrale Auflōsung (nm) } & $450-490 / 450-520$ (b) & $450-900 / 450-520(\mathrm{~b})$ \\
\hline & $520-600(g)$ & $520-600(9)$ \\
\hline & $630-690 \%$ & $630-6909$ \\
\hline & $760-890$ (nir) & $760-900$ (nir) \\
\hline & & \\
\hline & & \\
\hline Szenenabdeckung & $17 \times 17 \mathrm{~km}$ & $11 \times 11 \mathrm{~km}$ \\
\hline Orbithohe & $450 \mathrm{~km}$ & $681 \mathrm{~km}$ \\
\hline Inklination & 98* (sonnensynchron) & 98.1* (sonnensynchron) \\
\hline Anwendungsma0stab & $1: 5000 \cdot 1: 25000$ & $1: 5000 \cdot 1: 25000$ \\
\hline
\end{tabular}

Tab. 3: Ultrahochauflösende Satellitensysteme.

\subsection{Radar (Radio Detection And Ranging)}

Radarsysteme sind aktive Fernerkundungssysteme, d. h., sie messen die Laufzeit und die Intensität von ausgestrahlter und am Objekt (z. B. Bodenoberfläche, Gestein, Bebauung, Vegetation) reflektierter elektromagnetischer Strahlung im Mikrowellenbereich (vgl. Radarbereich in Abb. 1). Radarsensoren schauen bei ihren Aufzeichnungen aufnahmebedingt zur Seite (side-looking instruments). Der Anteil des Radarbildes, welcher am dichtesten am Nadir-Track (Oberflächennormalen im Aufzeichnungsmodus) ist, wird als „,near range“ bezeichnet. Der Bildteil, der am weitesten vom Nadir-Track entfernt ist, heißt „far range“. Bei der 
Bewegung des aktiven Sensors vom „near range“ zum „far range“ wächst der Einfallswinkel des Radarpulses an, deshalb ist es wichtig, zwischen Einfallswinkel bzw. Anstellwinkel des Sensors und lokalen Einfallswinkel zu unterscheiden. Hier kann sich ein erheblicher Unterschied aufgrund von Reliefunterschieden ergeben. Der Radarsensor misst nun die lineare Entfernung zwischen seiner Sendeantenne und dem Objekt. Diese Linie heißt „slant range“. Die wahre horizontale Distanz am Boden, die mit jedem im „slant range“ gemessenem Messpunkt korrespondiert, wird als „ground range“ bezeichnet. Ein Radarbild wird durch ausgesendete und rückgestreute Signale (transmitted and backscattered signals) erzeugt, bildet jeder gesendete „Radar-Puls“ ein Element im Radarbild ab, so wird das System als „Real Apertur Radar“ bezeichnet. Die räumliche Auflösung des Radarbildes in „slant range - Richtung“ und Azimuth-Richtung wird durch die Pulslänge und die Bündelbreite der Antenne (Öffnungswinkel) definiert. Aufgrund der unterschiedlichen Parameter, welche die räumliche Auflösung in „slant“ und Azimuth-Richtung bestimmen, ist die räumliche Auflösung in beiden Richtungen ungleich. Für die Interpretation von Radarbilder werden diese auf eine gleichmäßige Pixelgröße resampelt. Im Falle des ERS-1 SAR wird eine Pixelgröße von 12,5 x 12,5 m berechnet. Da die Länge einer Antenne auf einem Satelliten physikalisch begrenzt ist, ist auch die Apertur (Öffnungswinkel) begrenzt. Deshalb bedient man sich heute eines technischen Tricks, durch den die Apertur synthetisch erweitert wird. Die synthetische Verlängerung der Antenne wird dadurch erreicht, dass man die Vorwärtsbewegung der Aufzeichnungsplattform (Satellit, Flugzeug) sowie mehrere rückgestreute Signale eines Objekts zur Simulation einer verlängerten Antenne benutzt. Heute werden fast ausschließlich diese SAR-Sensoren (Synthetic Aperture Radar) eingesetzt.

Eine der wichtigsten Radarentwicklungen der letzten Jahre ist das Interferometrische SAR (InSAR). Das InSAR beruht auf der Messung von Phasenunterschieden von zwei benachbarten Orten mit bekannter Position. Das Ergebnis ist ein digitales Oberflächenmodell. Als wichtige InSAR-Anwendung der letzten Jahre ist die „Space Shuttle Topography Mission“ (SRTM) zu nennen, die an Bord des Space Shuttle im Februar 2000 an ca. elf Tagen die Erde umkreiste, um ein hochgenaues Oberflächenmodell der Erde zu erfassen.

Die Radarsysteme sind auf unterschiedlichen Plattformen (Satelliten, Flugzeuge, terrestrische Stationen) zu finden. Wichtige Radarsatelliten sind heute der European Remote Sensing Satellite (ERS-1 und ERS-2) oder der neue europäische ENVISAT-Satellit. Die Vorteile der Radarsysteme liegen in der Unabhängigkeit von Bewölkung und Beleuchtung. Hier sind sie den optischen Systemen in vielen Anwendungsbereichen und Landschaftszonen (Tropen) überlegen. 


\section{Flugzeuggestützte Systeme}

Neben den ultrahochauflösenden Satellitensystemen IKONOS und QUICKBIRD erleben die flugzeuggetragenen digital aufzeichnenden Systeme eine Renaissance. In der digitalen Photogrammetrie hat in den letzten Jahren die Koppelung digitaler Scanner an GPS-Systeme unter gleichzeitiger Einbindung von INSSystemen (inertiales Navigationssystem) zu hochgenauen digitalen multispektralen Bilddaten geführt. Diese Entwicklung wird mittelfristig die analogen Reihenmesskammern ablösen. Die Vorteile der digitalen Scanner liegen auf der Hand: digitale Datenaufnahme mit besserer radiometrischer Empfindlichkeit, direkte Integrationsfähigkeit in GIS sowie Verfügbarkeit echter 3-D-Fähigkeit durch Anwendung von GPS und INS. Damit werden durch diese Verfahren echte 3-D-Daten erzeugt, die in GIS-Systemen vorgehalten werden können. Kraus (2001) spricht diesbezüglich von einem Paradigma-Wechsel in der Photogrammetrie durch die Einführung des Laser-Scanning.

\subsection{Laser Scanning}

Laserscanner zeichnen die Laufzeitunterschiede für die Strecken zwischen Sensor und reflektierendem Objekt auf. Wenn die Position und der Abstrahlwinkel des Lasers (z. B. quer zur Flugrichtung) bekannt ist, können digitale Oberflächen (DOM's) berechnet werden. Laser Scanning hat sich dadurch zur ernsthaften Alternative zur stereoskopischen Höhenmessung aus Stereoluftbildern entwickelt. Das monochromatische Bild eines Laserscanners hat aber heute noch eine deutlich schlechtere geometrische Auflösung als ein Luftbild. Laserscanner werden deshalb heute in der Anwendung mit einer digitalen Kamera gekoppelt. Das Laser-Scanning gestattet auch dann die Objektrekonstruktion, wenn nur ein Aufnahmestrahl zur Verfügung steht (wichtig in bewaldeten oder eng bebauten Gebieten). Das Laser-Scanning gestattet weiterhin die Objektrekonstruktion auch in Gebieten ohne Textur, wo die Stereophotogrammetrie versagt. Durch die digitalen Scanner in Flugzeugen wird die Raumauflösung eines betrachteten Bodensegments weiter erhöht, sodass hier Daten entstehen, die eine vielfache räumliche Auflösung gegenüber den ultrahochauflösenden Satellitendaten aufweisen.

\section{Neue Auswerteverfahren für Fernerkundungsdaten}

Ein Grundproblem der digitalen Bildverarbeitung höchstauflösender Scanner ist, dass der integrierende Charakter (low-pass-Filterung) der Satellitenscanner mittlerer Auflösung verloren geht. Somit findet bei den höchstauflösenden Systemen keine Objektaggregation mehr statt. Dadurch kommt es nicht mehr zu einer Grauwerthomogenisierung, sondern die Objekte werden dann auf mehrere inhomogene Pixel aufgeteilt. Die neuen Sensor-Daten erfordern dadurch neue Aus- 
werteverfahren, die diesen Umstand der nicht mehr stattfindenden Grauwerthomogenisierung ausgleichen. Hier gilt es die nun vorliegenden Daten durch eine geeignete Vorstrukturierung von kontextbasierten Eigenschaften zu verarbeiten, damit ein automatisiertes Auswerten ermöglicht wird. Als zentrale Forderung gilt hier parallel zur Neuentwicklung von Sensoren die Erschaffung neuartiger Auswerteverfahren. Neue Sensoren und Auswerteverfahren werden neue Anwendungsbereiche nach sich ziehen. Sehr viel versprechende Ansätze sind in Segmentierungsverfahren, Multisensor- und der Multimethodenintegration zu sehen. Diese Ansätze werden ergänzt durch die Integration vorhandener Metadaten in geographische Informationssysteme. Erste Softwareentwicklungen (z. B. eCognition) bieten kommerzielle Software zur Segmentierung von hochauflösenden Bilddaten an. Insgesamt ist ein Zusammenwachsen von Bildverarbeitung und GIS zu erkennen. Diese Verschmelzung von Fernerkundung und GIS führt zu einer modernen angewandten Geoinformatik, in der zum ersten Mal ein integrierender Gesamtansatz für die Erfassung (Fernerkundung), Speicherung, Analyse und Weiterverarbeitung (GIS) der Geodaten gegeben ist.

Im Folgenden soll das Augenmerk auf drei wichtige Tendenzen in der zukünftigen Bildverarbeitung gelegt werden: Der Segmentierung, der Formcharakteristik typischer Landbedeckungstypen und der Hyperspektralanalyse.

\subsection{Segmentierung}

Unterschiedliche Studien haben die Beschränkung von pixelbasierten Klassifikationsverfahren für die Analyse von sehr hoch aufgelösten Bilddaten aufgezeigt (Blaschke 2000 c, d, Andresen et al. 2002). Zwar ist das Mischpixelproblem dieser Daten gering, jedoch hat die Variabilität und das Rauschen innerhalb quasihomogener Klassen stark zugenommen (vgl. Schiewe, Tufte \& Ehlers 2001). Dies bedingt Probleme hinsichtlich der zu bearbeitenden Datenmenge sowie des Detailgehaltes der Daten. Um die neuen Auswertungsanforderungen zu bewältigen, sind innovative, nicht allein grauwertorientierte Ansätze erforderlich, denn Objektform und Nachbarschaftsbeziehungen sind ebenso wie spektrale Eigenschaften, wichtige Merkmale zur Klassifikation bestimmter Nutzungsformen (Neubert \& Meinel 2002).

Die unbefriedigenden Ergebnisse der traditionellen Lösungsansätze mündeten in den Einsatz von regionen-basierten Verfahren (Schiewe \& Tufte 2002). Hierbei wird vor der eigentlichen Klassifikation versucht, die Daten in sogenannte Segmente (Objekte) zu zerlegen, innerhalb derer die Pixel zueinander hohe Ähnlichkeiten aufweisen (Pilz \& Strobl 2002).

Während traditionelle, multi-spektrale Klassifizierungsalgorithmen lediglich spektrale Ähnlichkeiten unabhängig von ihrem lokalen Auftreten berücksichtigen, gehen Segmentierungsansätze von der Hypothese aus, dass benachbarte Bildelemente derselben Klasse angehören. Diese Behauptung wird auf Grundlage von Homogenitäts- oder Heterogenitätsparametern bestätigt oder abgelehnt. Die ent- 
sprechenden Verfahren hierfür können punkt-, kanten- oder regionenbasiert arbeiten (Schiewe \& Tufte 2002).

Für Fernerkundungsanwendungen ist die segmentbasierte Software eCognition im Moment der vielversprechendste, kommerzielle Ansatz (Neubert \& Meinel 2002). Als erster Bearbeitungsschritt in eCognition erfolgt eine Segmentierung der Bilddaten zu homogenen Arealen. Für jedes dieser Segmente (Objekte) werden Objekt-Eigenschaften berechnet und in einer Datenbank abgelegt. Dabei können unter Nutzung von Daten unterschiedlicher Auflösung sowie von Daten unterschiedlicher Herkunft (z. B. zusätzliche GIS-Daten) mehrere Segmentierungsebenen angelegt werden. Im Vergleich zu pixelbasierten Klassifikationsansätzen, die nur die spektralen Eigenschaften nutzen, kennen die Bildobjekte bereits im unklassifizierten Zustand ihre Nachbarn und besitzen diverse Zusatzinformationen, wie z. B. Textur, Form, Beziehung zu Über- oder Unterobjekten. Das somit entstehende hierarchische Netzwerk von Bildelementen wird dem Bildgehalt auf unterschiedlichen Betrachtungsebenen bzw. Maßstäben besser gerecht (Neubert \& Meinel 2002). Anschließend erfolgt in eCognition eine Fuzzy-Klassifikation, welche auf den verschiedenen Objekteigenschaften in der Datenbank basiert. Auf der Basis der Objekteigenschaften wird zunächst ein Basisregelwerk in kleinen Testgebieten entwickelt, welches die Klassifikationsbedingungen der aufgeführten Klassen durch Definition von Zugehörigkeitsfunktionen enthält. Dabei sind Nachbarschaftsbeziehungen, Relationen zu Sub- bzw. Superobjekten und Segmenteigenschaften (z. B. Formindizes) neben den Grauwertinformationen als Klassenmerkmale nutzbar, wodurch eine größere Klassendifferenzierbarkeit ermöglicht und eine vollständigere Klassifikation erreicht wird (nach Andresen et al. 2002, Neubert \& Meinel 2002). Die Klassifikation von Bildobjekten entspricht zusätzlich viel besser der visuellen und kognitiven Zugangsweise des Menschen, wodurch bessere Klassifikationsergebnisse durch intuitive Klassengestaltung zu erreichen sind. Durch die Bildung von Bildobjekten ist auch eine bessere Integration der Klassifikationsergebnisse in ein vektorielles GIS-Umfeld möglich. Dies vor allem auch deshalb, weil der sogenannte salt-and-pepper-Effekt wegfällt (Pilz \& Strobl 2002). Eine der wichtigsten Vorraussetzungen für die Klassifikation von Fernerkundungsdaten mit Hilfe objektorientierter Ansätze ist die Segmentierung aussagekräftiger Objekte, die nur Pixel einer zusammengehörenden semantischen Klasse enthalten (Baatz \& Schäpe 2000, nach Andresen et al. 2002). Die weiteren Arbeiten richten sich deshalb auf eine Verbesserung der Segmentierung und der Analyse weiterer segmentbezogener Merkmale, die in die Klassifizierung eingehen können (Janoth et al. 2002). Eine weitere Aufgabe liegt in der Entwicklung von Regelwerken zur automatischen Klassifikation. Diese sollten die Eigenschaften verschiedener Fernerkundungssysteme berücksichtigen und auf unterschiedliche Gebiete übertragbar sein. Auch ist die Nutzung von zusätzlichem Wissen in Form thematischer Ebenen denkbar. So wird eine Fokussierung auf „wichtige” Kernklassen möglich (Andresen et al.). 


\subsection{Formcharakteristik typischer Landbedeckungstypen}

Im Gegensatz zum spektralen Informationsgehalt von Multispektraldaten wurde in den vergangenen 25 Jahren dem räumlichen Aspekt in digitalen Rasterdaten relativ wenig Beachtung geschenkt (Menz 1998). Landschaftsökologische Prozesse und Veränderungen unserer Umwelt sind aber häufig mit Änderungen von räumlichen Strukturen gekoppelt (Gasper \& Menz 1999). Heute ist allerdings unbestritten, dass die ökologische Wertigkeit eines Landschaftsobjektes neben seiner Nutzung auch durch seine Struktur (z. B. Fläche, Form) und Nachbarschaft (z. B. Umfang und benachbarte Nutzungen anderer Objekte beeinflusst wird. (zit. Borg \& Klisch 1999). Dementsprechend kann die quantitative Erfassung dieser Eigenschaften eine zusätzliche Information für die Bewertung ökologischer Prozesse darstellen (Borg \& Fichtelmann 1998). Zur konkreten Erfassung der Landschaftsstruktur wird in der Literatur eine Vielzahl unterschiedlicher Parameter beschrieben (Walz 2001). Wichtige Indizes sind in diesem Zusammenhang, neben zahlreichen anderen, der Dominance-Index, der Contagion-Index und der Shape Complexity-Index. Der Dominance-Index ermöglicht eine Aussage darüber, inwieweit die im Bild vorhandenen Klassen ähnliche relative Häufigkeiten besitzen oder stark von einer einzigen Klasse dominiert werden. Damit kann er als ein Biodiversitätsindex aufgefasst werden. Der Contagion-Index gibt den Grad der Aggregierung der im Bild vorhandenen Regionen bzw. der Zusammengehörigkeit von Pixeln der gleichen Klasse wieder. Er dient damit als Maß für die Zergliederung- bzw. Fragmentierung von Landoberflächen. Der Shape-ComplexityIndex ermöglicht eine Aussage über die Formkomplexität der im Bild vorhandenen Regionen. Er kann etwa dazu genutzt werden, den Einfluss menschlicher Aktivität in bestimmten Gebieten zu erfassen (O’Neill et al. 1988 in Gasper \& Menz 1999). Dabei sind verschiedene Betrachtungsebenen zu berücksichtigen. Einerseits können die einzelnen Landschaftselemente untersucht werden. Konkrete Maße lassen sich dabei für die Form der jeweiligen Elemente, den Verlauf der Ränder (Kanten) und für die Lagebeziehung einzelner Elemente zueinander ableiten. Andererseits kann das Landschaftsmosaik, das sich aus den einzelnen Elementen zusammensetzt, in seiner Gesamtheit beschrieben werden. Dabei geht es im Wesentlichen um Auswertungen zur Vielfalt der Nutzungseinheiten, ihrer Verteilung und zur Anordnung linienhafter Elemente. Anstatt Maße für die gesamte Landschaft mit allen ihren Nutzungsklassen zu berechnen, kann dies auch für einzelne Nutzungsklassen geschehen (Walz 2001). Typischerweise werden Landschaftsmaße aus Fernerkundungsdaten gewonnen, die zuvor einer Klassifizierung unterzogen werden müssen (O’Neill et al. 1988 in Menz 1998), in geringem Umfang werden sie auch direkt aus Spektraldaten abgeleitet (De Jong et al. 1995 in Menz 1998). Landschaftsmaße und Fernerkundung sind laut Menz 1998 ein wichtiges „Tool“, um Raummuster und deren raumzeitliche Veränderung in den verschiedensten Maßstäben bzw. Größen quantitativ zu erfassen. Die Kriterien zur Qualitätsbewertung von Formindizes sind ihre Größen-, 
Richtungs- und Maßstabsinvarianz (Shapiro 1985) sowie die weitgehend eindeutige Interpretierbarkeit (Borg \& Fichtelmann 1998).

Das heißt (O’Neill et al. 1988):

- Der jeweilige Index sollte gleiche Strukturen in der Landschaft mit identischen Werten beschreiben.

- Der Index solle unabhängig und unkorreliert von anderen Indizes sein.

- Indizes sollten skaleninvariant sein, d. h., sie sollten unabhängig von der absoluten Fläche des Untersuchungsgebietes sein.

Die zahlreichen Indizes, die im Bereich der Landschaftsökologie entwickelt wurden, weisen allerdings noch einige Unstimmigkeiten und Ungenauigkeiten auf. Vergleiche unterschiedlicher Arbeiten sind aufgrund der Anwendung verschiedener Daten und Formeln bisher kaum möglich. Für die weitere Forschung ist die Untersuchung von Zusammenhängen zwischen den Ergebnissen der Landschaftsmaße, insbesondere bei einer räumlichen Differenzierung der Werte, und landschaftsökologischen Prozessen notwendig (Gasper \& Menz 1999). Grundsätzlich ist zwar festzustellen, dass es enge Zusammenhänge zwischen Landschaftsstruktur und biotischer Vielfalt gibt, Zusammenhänge zwischen der Habitateignung und Patchgröße und -form (Patch: je nach Betrachtungsebene das kleinste als weitgehend homogen anzusehende Einzelelement einer Landschaft) sind aber bisher nur für eine eng begrenzte Anzahl gut erforschter Schlüsselarten hergestellt worden. Für eine Quantifizierung müßten außerdem vergleichbare Untersuchungen in einer größeren Zahl von Gebieten in unterschiedlichen Naturräumen und detaillierte landschaftsökologische Analysen angestellt werden. Bei den zukünftigen Untersuchungen müssen konkrete, überschaubare Fragestellungen im Vordergrund stehen, um die Zusammenhänge zwischen Struktur und Funktion wenigstens in Teilbereichen weiter zu untermauern. Ganz konkrete Beispiele für die Verwendung der einzelnen Indizes fehlen in der Literatur nach wie vor, selbst in Standardwerken zum Umgang mit der Landschaftsstruktur (Walz 2001). Neben dem Einsatz von Fernerkundungsdaten ist die Einbeziehung von zusätzlichen Geo-Informationen zur ökologischen Zustandsbeschreibung wieterhin notwendig, insbesondere die Verschneidung mit zusätzlichen landschaftsökologischen Aussagen zur Überprüfung von funktionellen Zusammenhängen (Walz 2001). So können die spezifischen Vor- und Nachteile einzelner Datenbasen ausgeglichen und um Inhalte gegenseitig ergänzt werden.

Die neuen Sensoren und Techniken liefern immer höher auflösende Geodaten von den Objekten. Übersteigt die Objektgröße die Pixelauflösung, so kommt es nicht mehr zur homogenisierenden Grauwertglättung. Eine pixelbasierte Klassifikation wie bei den herkömmlichen mittelhoch auflösenden Multispektralscannern ist nun nicht mehr ratsam. Neue Methoden der Bildverarbeitung nutzen deshalb sogenannte kontextbasierende Verfahren zur Bildauswertung. Hier ist also nicht mehr der Pixelbezug, sondern der Objektbezug dominant, obwohl das Pixel die kleinste räumliche Einheit bleibt und somit auch Basis jeglicher Segmentierung ist. Festzuhalten ist hier allerdings, dass Standardmethoden zur operationellen Analyse von höchstauflösenden Satellitendaten noch zu entwickeln sind. Hier liegt ein ho- 
her Forschungsbedarf im Bereich der angewandten Bildanalyse höchstauflösender Bilder. Die skizzierten Entwicklungen von den neuen Sensoren über neuartige Auswertemethoden, werden zu neuen Anwendungsfeldern führen. Dies ist insofern von Bedeutung, da sich das Herangehen an die Informationsextraktion aus Satellitenbildern noch stärker in Richtung wissensbasierter Bildanalyse verschieben wird. Somit wird die Integration von GIS und Fernerkundung in die einzelnen Fachbereiche immer wichtiger. Das spezifische Fachwissen wird im Umfeld einer kontextbasierten Interpretation von Satellitenbildern neues Gewicht erhalten. Dies lässt sich leicht am Beispiel einer Wald- bzw. Forstkartierung mit Satellitenbildern belegen. War es bei den pixelbasierten Ansätzen (z. B. Maximum-Likelihood) auch einem fachspezifisch (z. B. Forst, Geologie, Meteorologie, etc.) nicht ausgebildeten Bildverarbeiter möglich, eine Klassifikation zu erstellen, wenn ihm nur genügend Testflächen für eine Satellitenszene vorlagen, so ist ihm dies heute in einer multi-skalaren Bildsegmentierung nicht so einfach möglich. Das fachspezifische Wissen über Waldstrukturen, Vergesellschaftungen, Baumtexturen etc. lässt sich in das Voranschreiten einer Bild-Segmentierung einbauen. Das endgültige Klassifikationsergebnis kann somit schrittweise interaktiv verbessert werden bzw. hängt direkt vom „Vorwissen“ über den Zielraum ab. Die Erzeugung regionalisierter Segmentierungsebenen für höchst-auflösende Bilder ist also für sich zunächst ein Problem. Die im geowissenschaftlichen Maßstabsbereich relevanten Objektgruppen lassen sich nun immer differenzierter abbilden. Bei der klassischen Bildanalyse wurden für Landnutzungsklassifikationen via „Landsat“ nur wenige Klassen ausgeschieden. Heute ist hier eine sprunghafte Erhöhung der thematischen Tiefe zu beobachten. Wie kann man sich nun dieser räumlichen Heterogenität annähern? Diese Frage bildet die Grundlage der modernen Landschaftsstrukturanalyse mittels modernen Fernerkundungsmethoden in den nächsten Jahren. In einem Bildausschnitt oder einer ganzen Szene sind Landschaftseinheiten in horizontaler Sicht leicht zu separieren. Ein sprunghafter Wechsel in den spektralen Eigenschaften oder eine deutliche Veränderung gewisser Formparameter basieren auf homogenen Einheiten, die sich gegenseitig abgrenzen. Der Heterogenitätsgrad ist also bestimmt durch die Anzahl homogen erscheinender Einheiten. Die zunächst erkennbare horizontale Anordnung dieses Musters (patch mosaics oder pattern) ist sehr stark maßstabsabhängig. Der Maßstabsbereich kann unser Muster (pattern) in raumzeitlicher Betrachtung sehr stark verändern. Aus der Fernerkundungssicht gibt der Maßstab jene Zeitspanne oder räumliche Erstreckung an, über die hinweg ein Signal (spektrales Muster, Textureigenschaft, etc.) einheitlich bleibt, also zu homogenen Einheiten in der Klassifikation führt. Der gewählte Maßstabsbereich bestimmt somit die Grenzen in vertikaler und horizontaler Bildbetrachtung (Allen \& Star 1982).

\subsection{Hyperspektralanalyse}

Die letzten Ausführungen konzentrierten sich insbesondere auf das Problem räumlich hochauflösender Sensoren. Wie steht es aber mit der in den letzten 
Jahren zunehmenden Steigerung der spektralen Auflösung? Wurde früher bei zu hoher spektraler Auflösung das „Auf und $A b^{“}$ des Kurvenverlaufs eines Reflexionsspektrums als „Rauschen“ empfunden und als Störgröße eliminiert, so wissen wir heute, dass sich hinter dem kontinuierlichen Verlauf eines objektbezogenen Reflexionsspektrums auch objektspezifische Information verbirgt. Die Entwicklung der „Hyperspektralanalyse“ hat in den letzten Jahren ein großes Anwendungspotential für quantitative Betrachtungen aus der Fernerkundung heraus dargelegt. Die Abbildung 3 zeigt die Grundlagen der Refelexionsspektrometrie. Der Schritt von der überwiegend deskriptiven Fernerkundung (was ist wo?) zur quantitativen Fernerkundung erweitert die Anwendungsmöglichkeiten enorm (vgl. Precision Farming, Vegetationsmonitoring, Lagerstättenanalyse). In der hoch technisierten Landwirtschaft ist es heute möglich, mittels Fernerkundung Inhaltsstoffe (z. B. Stickstoff) bzw. den Versorgungsgrad der Pflanzen zu ermitteln (Erasmi 2003). In der Geologie ermöglichen umfangreiche „Spektralbibliotheken“ eine Verbesserung der Kartierung von Gesteinsflächen bis hin zur Mineralbestimmung. Diese Verfahren werden überwiegend in der Exploration von Lagerstätten eingesetzt. Dabei ist der Übergang von hyperspektralen Labormethoden bis zu geländegestützten Messungen und deren Abgleich mit flugzeug- bzw. satellitengestützten Systemen fließend. Diese Anwendungen sind aber noch weit von einem operationellen Einsatz entfernt und liegen zurzeit nur als Fallstudien vor, die aber ein großes Potenzial dieses Fernerkundungszweigs aufweisen.

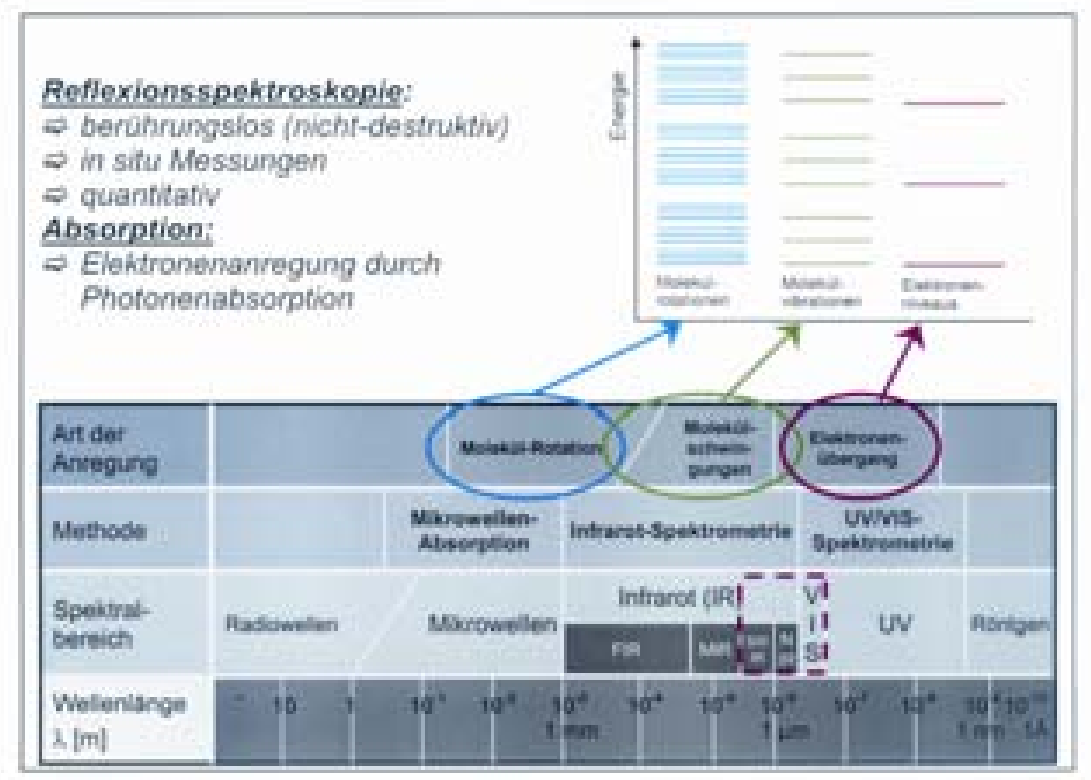

Abb. 3: Grundlagen der Reflexionsspektrometrie, Quelle: Erasmi, S., 2003. 


\section{Schlussbetrachtung und Tendenzen}

Die neuartigen Sensoren und Methoden der Fernerkundung stoßen in Anwendungsgebiete vor, die ehemals der Luftbildinterpretation vorbehalten waren. Dadurch ist es ohne aufwendige Luftbildbefliegung und deren Planung möglich, Bilder von der ganzen Erde in hoher Auflösung zu bekommen. Das System Erde wird somit erfassbar. Dies verleiht dem Fachwissenschaftler eine hohe Flexibilität in der Durchführung seiner Projekte und führt zu einem steigenden Einsatz von Fernerkundungsdaten in fast allen Disziplinen. Dies ist auch wünschenswert, wenn man sich vorstellt, dass die überwiegende Zahl der Fernerkundungsdaten ungenutzt in den Datenarchiven liegt. Die Bearbeitung neuer Anwendungen (wie Biotopkartierungen, Stadtkataster, Monitoring von Landschaften und Change Detection) wird den Bedarf an hochauflösenden Daten weiter steigern, aber auch die Entwicklung neuer Methoden erfordern. Wichtig ist hier, dass Methoden entwickelt werden, die sich als Standardverfahren etablieren können, um in Breite wahrgenommen zu werden.

Der Trend geht hier in Richtung komplexer Sensorsysteme bzw. multitemporaler (Change detection) und multisensoraler Betrachtung der Erde. Die multitemporalen Sichtweisen der Erde mittels Fernerkundung sind für die Geowissenschaften ein mächtiges Werkzeug zur Ableitung von Veränderungen auf unserer Erde (vgl. Abb. 4). Erst durch die wiederholende Betrachtung unserer Erde werden tiefgreifende Veränderungen im System sichtbar. Die Abbildung 4 zeigt tiefgreifende Veränderungen in den Regenwäldern Indonesiens in kurzer Zeitspanne. Neben der zeitlichen Dokumentation des Eingriffs können auch die Flächen schnell und objektiv quantifiziert werden. Das Erdsystem ist somit vielseitig und ganzheitlich in hoher zeitlicher und räumlicher Auflösung erfassbar.

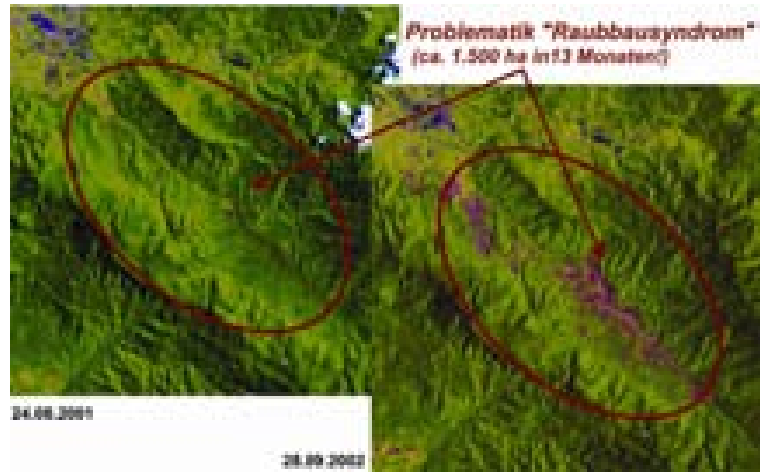

Abb. 4: Landsat ETM 741 (RGB) Change Detection des Waldrückgangs in Zentral Sulawesi, SFB552 STORMA.

Mit der Forderung einer multitemporalen und multisensoralen Betrachtung unserer Erde einher geht die Forderung nach besserer Kalibrierung der Sensoren. 
Weiterhin ist es nötig, Methoden und ihre Ergebnisse übertragbar und leicht verständlich für potenzielle Anwender zu gestalten. Die viel angepriesene Integration von Daten, Methoden, Modellen, Prozessen, usw. ist zwar heute schon möglich, in der Realität aber noch nicht häufig verwirklicht. Diese Integration dürfte die Hauptaufgabe der nächsten Jahre sein, um das Anwendungspotenzial der Fernerkundung noch weiter zu steigern.

\section{Literatur}

Allan, T. F. H. \& Starr, T. B. (1982): Hierachy - Perspectives of ecological complexity, Chicago

Andresen, T., Mott, C., Schüpferling, R., Zimmermann, S. \& Schneider, T. (2002): Objektorientierte Analyse von Fernerkundungsdaten zur Erfassung aquatisch/terrestrischer Parameter. In: Blaschke, T. (Hrsg.): GIS und Fernerkundung: Neue Sensoren - Innovative Methoden, Heidelberg, 222-232

Baatz, M. \& Schäpf, A. (2000): Multiresolution Segmentation - an optimisation approach for high quality multi-scale image segmentation. In: Strobl, J. et al. (Hrsg.): Angewandte Geographische Informationsverarbeitung XII. Beiträge zum AGIT-Symposium Salzburg 2000, Karlsruhe, 12-23

Blaschke, T. (2000d): Objektextraktion und regelbasierte Klassifikation von Fernerkundungsdaten: Neue Möglichkeiten für GIS-Anwender und Planer. In: 5. Symposium „Computergestützte Raumplanung“ - CORP2000, 153-162

Blaschke, T. (2000c): Ohne Salz und Pfeffer. Objektorientierte Bildanalyse - eine Revolution in der Fernerkundung. In: GeoBIT 2/2000, 30-32

Borg, E., \& Fichtelmann, B. (1998): Vergleichende Analyse von Formindizes zur Charakterisierung von Landschaftsobjekten unter ökologischen Aspekten, ZPF, Heidelberg, 66 (4): 108-119

Borg, E. \& Klisch, A. (1999): Untersuchungen zu zweidimensionalen Strukturinformationen am Beispiel von Waldobjekten im Warnow-Einzugsgebiet, VGI, Bd. 87, 4

Erasmi, S. (2003) : Optische Fernerkundung zur teilflächenspezifischen Zustandsbeschreibung von Winterweizen, Göttinger Geographische Abhandlungen, Heft 110

Gasper, P. \& Menz, G. (1999): Landschaftsmaße zur Bestimmung der räumlichen Strukturen in klassifizierten Satellitenbildern, Publikationen der Deutschen Gesellschaft für Photogrammetrie und Fernerkundung, Bd. 7, 333-340

Janoth, J., Eisl, M., Klaushofer, F. \& Luckel, W. (2002): Segmentbasierte Verfahren zur Veränderungsanalyse und Waldklassifizierung mit hochauflösender Satellitendaten. In: Blaschke, T. (Hrsg.): GIS und Fernerkundung: Neue Sensoren - Innovative Methoden, Heidelberg, 98-107 
Jong, S. M. D. \& Burrough, P. A. (1995): A fractal approach to the classification of mediterranean vegetation types in remotely sensed images, Photogrammetric Engeneering \& Remote Sensing 61 H. 8, 1041-1053

Kappas, M. (1994): Fernerkundung - nah gebracht. Leitfaden für Geowissenschaftler, Dümmler Verlag, Bonn, 207 S.

Kappas, M. (2001): Geographische Informationssysteme. Westermann Verlag, Das Geographische Seminar, Braunschweig, 317 S.

Kraus, K. (2001): Laser-Scanning - Ein Paradigma-Wechsel in der Photogrammetrie. In: Publikationen der Deutschen Gesellschaft für Photogrammetrie und Fernerkundung, Band 10, 13-22

Menz, G. (1998): Landschaftsmaße und Fernerkundung - neue Instrumente für die Umweltforschung, Geographische Rundschau 50, H. 2, 106-112

Neubert, M. \& Meinel, G. (2001): Segmentbasierte Auswertung von IKONOSDaten. In: ZGIS \& DGPF (Veranstalter): Fernerkundung und GIS. Neue Sensoren - Innovative Methoden, Workshop im Vorfeld der AGIT 2001. Salzburg, 3./4. Juli 2001, 22-23

Neubert, M. \& Meinel, G. (2002): Segmentbasierte Auswertung von IKONOSDaten - Anwendung der Bildanalyse-Software eCognition auf unterschiedliche Testgebiete. In: Blaschke, T. (Hrsg.): GIS und Fernerkundung: Neue Sensoren - Innovative Methoden, Heidelberg, 108-117

O’Neill, R. V., Krummel, J. R., Gardner, R. H., Sugihara, G., Jachson, B., DeAngelis, D. L., Milne, B. T., Turner, M. G., Zygmunt, B., Christensen, S. W., Dale, V. H., Graham, R. L. (1988): Indices of landscape pattern, Landscape Ecology 2, 63-69

Pilz, H. \& Strobl, J. (2002): Versiegelungsanalyse auf Basis digitaler Farborthofotos mittels objektorientierter Bildverarbeitung. In: Blaschke, T. (Hrsg.): GIS und Fernerkundung: Neue Sensoren - Innovative Methoden, Heidelberg, $159-168$

Shapiro, L. G. (1985): Recent progress in shape decomposition and analysis. In: Kanal, L. N., Rosenfeld, A. (eds.): Progress in Pattern Recognition 2, Elsevier, Amsterdam, 113-123

Schiewe, J., Tufte, L. \& Ehlers, M. (2001): Potential and problems of multi-scale segmentation methods in remote sensing. In: GeoBIT/GIS 6, 34-39

Schiewe, J. \& Tufte, L. (2002): Potenzial regionen-basierter Verfahren für die integrative Auswertung von GIS- und Fernerkundungsdaten. In: Blaschke, T. (Hrsg.): GIS und Fernerkundung: Neue Sensoren - Innovative Methoden, Heidelberg, 42-52

Walz, U. (2001): Charakterisierung der Landschaftsstruktur mit Methoden der Satelliten-Fernerkundung und der Geoinformatik, Berlin 


\title{
Die überwundene Schwerkraft - eine halbe Milliarde Jahre Wirbeltier-Evolution
}

\author{
Annette Broschinski
}

Die Fragestellung ,-- was bewegt die Welt?“ kann in besonderem Maße auf die Entwicklung der Wirbeltiere angewendet werden: Zum einen interessiert die Bewegung des ganzen Tieres relativ zu seiner Umgebung, also die Fortbewegung, zum anderen die kleinmaßstäbigen Bewegungen ohne Fortbewegungskontext, aber dafür mit gestaltändernden Auswirkungen, wobei hier der Schwerpunkt auf die wichtigste dieser Bewegungen gelegt wird, den Nahrungserwerb.

Aus dem Blickwinkel der Kenntnis vieler heutiger Säugetiere betrachtet, scheinen diese Aspekte leicht erkennbar zu sein: Beine bewegen sich gegen das Widerlager eines Körpers, Kiefer bewegen sich hin und her, um Nahrung zu zerbeißen. Es bedarf jedoch eines großen Zeitsprungs zurück zu den Anfängen der Wirbeltierentstehung im Verlauf des Kambriums respektive bereits davor, einer Zeit mit kleinen, spindelförmigen bis flach gebauten „Fischartigen“ ohne Flossen und ohne Kiefer, um zu verstehen, dass die heutigen Beobachtungen auf die Vorläuferstadien nicht immer klar anwendbar sind. Oft existiert nicht einmal ein rezentes Analogon für frühe Formen in der heutigen Lebewelt - und dies gilt für die verschiedensten evolutiven Ebenen. Um so wichtiger ist es daher, neben dem systematisch-taxonomischen Aspekt der Artabgrenzung und Verwandtschaftsanalyse stets den funktionsmorphologischen Aspekt im Auge zu behalten, der ja das Erscheinungsbild der Tiere maßgeblich beeinflusst und letztlich Erklärungsmöglichkeiten für die gestaltlichen Veränderungen liefert. 
Wirbeltierskelette sollen hier daher als Festteile von Konstruktionen (= Tragwerken) verstanden werden, die zu allen Zeiten fortschreitenden Veränderungen unterlagen, welche zu funktionsmorphologischen Verbesserungen führen konnten: Knapp 500 Millionen Jahre Optimierungsprozesse führten zu immer neuen Wirbeltiergruppen. Die Betonung liegt dabei auf „konnten“, nicht auf einer denkbaren Zwanghaftigkeit, wie der Fortbestand vieler ursprünglicherer Baupläne in den einzelnen Großgruppen beweist (Neunaugen, Echsen, u. v. m.). Einzelne Optimierungen bildeten sich heraus, aber Bewährtes blieb häufig gleichermaßen erhalten.

Das knorpelige und/oder knöcherne Skelett der Wirbeltiere stützt den Körper und dient als Ansatzfläche für Muskulatur im weitesten Sinne (Entspringen von Muskeln, Inserieren am Zielpunkt). Die Problematik von „Bewegung“ macht es dabei oft schwierig, die sonst grundsätzlich geltenden statischen Gesetze für Tragwerkskonstruktionen anzuwenden: Kräftegleichgewicht gilt für ruhende Konstruktionen. Es kann daher nur eine stark vereinfachte Beschreibung und Deutung der einzelnen Wirbeltiertypen erfolgen, um einen ,roten Faden“ durch die komplexen Entwicklungsabläufe zu gewinnen. Dabei tauchen unweigerlich zunehmend physikalische Erklärungsmuster aus der Festkörpermechanik auf - wie beispielsweise die Hebelgesetze - die mit den natürlichen Baumaterialien der Wirbeltiere in Einklang gebracht werden müssen (Knochen, Knorpel, Sehnen, Ligamente, u. v. m.).

Wie alles Leben auf der Erde stammen auch die Wirbeltiere aus dem Wasser. Sie leiten sich von einer Gruppe der Chordaten her, rückenmarktragende, längliche, kleine Meerestierchen (wenige $\mathrm{cm}$ ), deren entwicklungsgeschichtliches Markenzeichen auch zum Markenzeichen der Wirbeltiere wird: eine versteifte Körperlängsachse. Diese zunächst knorpelig gebildete Struktur (Chorda) ermöglicht denjenigen Vertretern der Gruppe, die sie vollständig ausbilden, eine innere Stabilisierung des Körpers bei zusätzlicher Schaffung von neuen Muskelansatzstellen und gleichzeitiger Elastizität in der Reaktion auf Belastungen von außen. Fossile Funde aus dieser Gruppe, die dem heute noch lebenden Vertreter dieses Bauplans (Lanzettfischchen) sehr ähneln, sind beispielsweise aus dem Mittelkambrium von Kanada bekannt (Burgess Shale: Gattung Pikaia). Echte Flossen sind bei beiden Formen nicht entwickelt, und der Versteifungsgrad der Körperlängsachse ist durch die Chorda dorsalis bereits so groß, dass für die Fortbewegung ein separater Flossensaum nötig ist, der in rhythmischen Wellen für die Fortbewegung der Tiere sorgt. Am spitzen Kopfende befindet sich die Mundöffnung, die klein und kieferlos ist und einen Kranz winziger Tentakeln ausbildet: Es handelt sich also um Suspensionsfressen, die ursprünglichste aller Arten von Nahrungserwerb. Für beide Bewegungsarten sind also keinerlei Hartteile zuständig, es können nur kleine und kleinste Nahrungspartikel gefressen werden, die frühesten erdgeschichtlichen Vertreter müssen zudem langsam gewesen sein und vor allem kleinwüchsig.

Die ersten echten Wirbeltiere - heute „Schädeltiere“ -, die wir als „Fische“ bezeichnen, obwohl sie mit den heutigen Fischen gemeinhin nichts zu tun haben, sind durch disartikulierte Hartteile überliefert: Panzer aus einer Vielzahl von Ein- 
zelschuppen sind aus oberkambrischen Sedimenten Wyomings bekannt. Kurz darauf im Ordovizium auftretende, vollständig erhaltene Exemplare geben uns ein Bild von jenen ersten Wirbeltieren. Es handelte sich um spindelförmige kleine Wesen, die sich durch eine ausgeprägte, äußere Hartteilpanzerung auszeichnen, die aus zahlreichen kleinen Einzelelementen besteht. Es handelt sich hierbei um Schuppen, jedoch nicht im heute für Knochenfische gültigen Sinne, sondern um die klassische Urform. Sie besteht i. d. R. aus drei Schichten, einer knöchernen Basis, einer mittleren Schicht aus einem zahnbeinartigen Material („Dentin“ sensu lato) und einer dünnen Deckschicht aus zahnschmelzartigem Material („Enameloid“ sensu lato). Alle drei Schichten sind dabei prinzipiell aus demselben Material gebaut, dem Hydroxylapatit (und diversen, sehr ähnlichen Derivaten mit FluorAnhängseln etc.). Knochen stellt dabei die lockerste Bauweise dieser Substanz dar, „Dentin“ eine wesentlich dichtere und „Enameloid“ schließlich die dichteste. Die Panzerung durch die kleinen, dreilagigen Einzelschuppen verlief bei den frühesten „Fischartigen“ flächendeckend von der Schnauzenspitze bis zum Schwanzende. Der Kopf war leicht abgerundet und zudem niedrig (dorsoventrale Abflachung), wohingegen die Schwanzregion eine seitliche Verschlankung ausbildete (laterale Abflachung). Flossen waren nicht entwickelt. Die Tiere lebten daher wohl stark bodenbezogen (flacher Kopf) und bewegten den hinteren Teil des Körpers seitlich hin und her (= laterale Undulation), um sich fortzubewegen (Abb. 1, 1.1). Sicherlich limitierte die starke Panzerung ausgeprägte Bewegungen dieses Typs. Der seitlich abgeflachte Schwanz übernahm anscheinend bereits eine zentrale Rolle beim Bewegungsantrieb. Die zentrale, meist schmale Maulöffnung erlaubte wenig mehr als ein erweitertes Suspensionsfressen. Die gut gepanzerten Tiere waren sicherlich recht gut gegen Fressfeinde geschützt, ansonsten übernahmen auch hier die Hartteile nur eine untergeordnete Rolle für die Bewegungen: Der am caudalen Pol leicht verbreiterte, seitlich abgeflachte Schwanz erleichterte die laterale Undulation ein wenig, ansonsten waren diese Tiere träge, partikelfressende Meeresbodenbewohner, die zwar geringfügig größer wurden als die ungepanzerten Ur-Lanzettfischchen, ansonsten jedoch keinerlei Potential für ein schnelleres Schwimmen oder eine Größenzunahme mitbrachten.

Interessant ist auch, dass gerade die Eigenbezeichnung der Wirbeltiere, die sich ja von Wirbeln herleitet, in diesem frühen Stadium nur schlecht nachweisbar ist. Man weiß durch die seltenen, etwas besser erhaltenen Fischfossilien aus diesen extrem alten Sedimenten, dass neben der Chorda bzw. um diese herum gelenkig miteinander verbundene, knorpelige Stützelemente ausgebildet waren, die zudem in rückenseitige und bauchseitige Verlängerungen übergingen: die ersten Wirbel. Sie ummantelten die Chorda. Durch den segmenthaften Charakter waren sowohl Stabilität als auch eine verbesserte Beweglichkeit in einer völlig neuartigen Kombination die Folge. Allerdings lagen alle diese frühen Wirbelsäulen inmitten der zuvor geschilderten Knochenpanzerung, was die Identifikation oft unmöglich macht. Knochen ist gut erhaltungsfähig, Knorpel dagegen erhält sich nur in Ausnahmefällen. Insbesondere bei Vorhandensein der harten Enameloid-Kappen 
der Schuppen fällt es stets schwer, etwaige Reste von knorpeligen Strukturen überhaupt zu erkennen.

Auffällig schnell tritt bei anderen dieser Fossilien ein abgewandeltes Erscheinungsbild dieses Typs in Erscheinung. Während der hintere Teil der Fischkörper oberflächlich betrachtet gleich aussieht, besteht der vordere Teil abweichend nicht aus einer Vielzahl kleiner Schuppen, sondern aus wenigen großen Platten (Abb. 1, 1.2). Diese immense Erhöhung der Rigidität, also die von außen gebildete Versteifung der vorderen Körperhälfte durch einen festen äußeren Panzer, wird bei genauerer Betrachtung am hinteren Pol durch eine stärker differenzierte Schwanzregion ergänzt, wobei in den verschiedenen Gruppen dieser Fische (z. B. den Heterostraci) unterschiedlich geformte, sehr ursprüngliche Schwanzflossen entstehen. Da diese weiterhin kleinformatig gepanzert waren, verfügten die Tiere im Gegensatz zum Kopf-Rumpf-Pol über die nötige Beweglichkeit, um einen lateralen Schwanzflossenschlag zu generieren, der eine Vorwärtsbewegung erzeugte (Abb. 1, 1.3). Allerdings fehlen weitere Flossen neben der Schwanzflosse, was die tatsächliche Beweglichkeit einschränkte und eher auf ein nur kurzzeitiges Verlassen des Meeresgrundes rückschließen lässt. Schließlich musste eine reine Vorwärtsbewegung ohne der Schwerkraft entgegenwirkende Komponenten zu einer geringen Manövrierfähigkeit und kurzen Schubphasen beim Schwimmen führen.
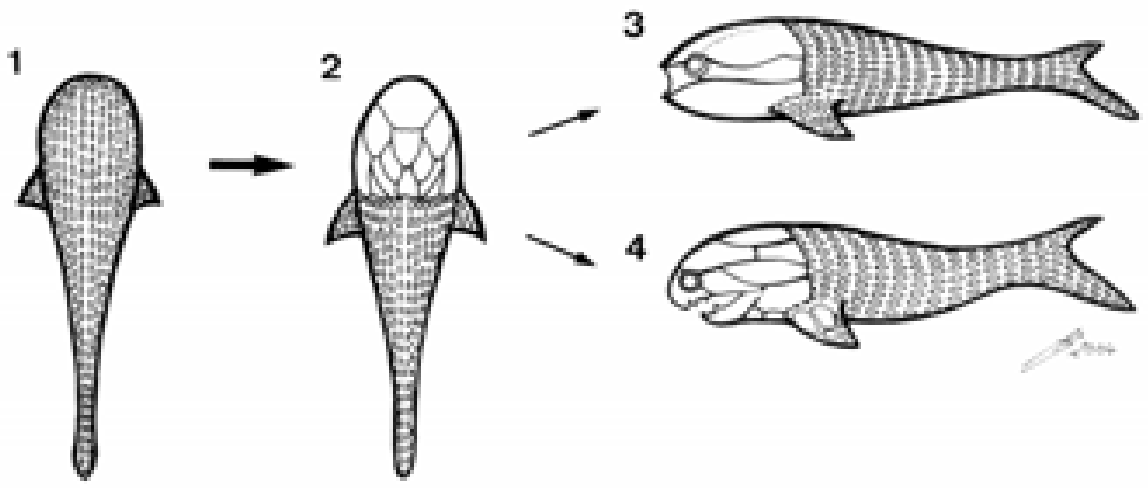

Abb.1: Strategien der Entwicklung früher Fischpanzer. 1.1: Ursprünglichster Form der Panzerung, kleine Schuppen von rostral bis caudal. 1.2: Entwicklung des Kopf-Brust-Panzers aus großen Platten, Beibehaltung des kleinformatigen Schuppenpanzers im binteren Bereich des Körpers. 1.3: Beibehaltung und Optimierung des Prinzips von 1.2 bei den kieferlosen Panzerfischen (Agnatha). 1.4: Beibehaltung und Optimierung des Prinzips von 1.2 in Kombination der Evolution des Kieferapparats bei den kiefertragenden Panzerfischen (Placodermi). 1.1 konnte eine undulierende Bewegung fast der gesamten Körperachse vollziehen, 1.2 bis 1.4 waren durch die vordere Versteifung auf ein nach binten verlagertes Oszillieren angewiesen. Nicht maßstäblich. 
Die Mundöffnung war weiterhin klein und schmal und begrenzte daher das Nahrungsspektrum auf kleinwüchsige Organismen und Partikel. Allerdings fällt bei einigen Arten dieser ursprünglichen Fische die Ausbildung sogenannter Oralplatten auf, transversal verbreiterter, beweglicher Zusatzelemente auf der Unterseite der Mundöffnungen. Sie ergaben einen ähnlichen Effekt wie die erst viel später und bei anderen Fischgruppen auftretenden, echten Unterkiefer: Auf- und Abbewegung dieser Platten konnten zum Einfangen und zum Festhalten von kleinen Organismen genutzt werden respektive zum lückenlosen Schließen des Mauls.

Bei den weiterhin vielschuppig gepanzerten Fischartigen trat im Silur und Devon des weiteren eine Gruppe auf (Thelodontia), die neben der Schwanzflossenregion auch erstmals kleine Brustflossen- und Analflossen-Fortsätze ausbildet. Diese Fortsätze waren allerdings nicht gelenkig mit dem Körper verbunden, sondern stellten zunächst starre Auswüchse dar. Durch sie trat nun erstmals eine von vorn her Auftrieb erzeugende Struktur auf, die den hinten kommenden Bewegungsimpuls durch leichte Aufwärtsschrägstellung der Brustflossen nicht nur in eine vorwärts gerichtete, sondern in eine leicht nach oben gerichtete Bewegung führte und damit der Schwerkraft entgegenwirken konnte.

Aus paarigen Kiemenbogenelementen, die zu je zwei Elementen von Anfang an gelenkig miteinander verbunden waren, bildeten sich etwa im unteren Silur schließlich Kiefer. Die beiden Oberkieferelemente nahmen dabei im Laufe der Weiterentwicklung mehr oder weniger festen Kontakt zu den Schädelelementen auf. Ein gegen den Schädel beweglicher Unterkiefer (die beiden unteren der vier Elemente) wurde ein echtes Erfolgsmerkmal aller weiteren Wirbeltiere, von denen nur ganz wenige Gruppen im Laufe der Evolution sekundär abweichen sollten (Röhrennasenfische, Ameisenbären, etc.). Die Existenz von Kiefern ermöglichte schlagartig zwei neue Wege des Nahrungserwerbs: Zum einen das sogenannte „suction feeding“, also das Hineinziehen von kleineren Beutetieren in die Maulregion durch Erzeugen eines Unterdrucks durch Abpressen des Wasserstroms durch die Kiemen hinten bei anschließendem Totalverschluss des Mauls vorn, zum anderen auch das Erbeuten größerer Tiere, sodass die ersten kiefertragenden Fische zwar noch das klassische Aussehen „vorn großflächig gepanzert, hinten beweglich durch kleine Schuppen“ der kieferlosen Panzerfische fortsetzen (Abb. 1, 1.4), im Gegensatz zu jenen jedoch erstmalig große Formen hervorbrachten wie etwa die devonischen Gattungen Dinichthys oder Dunkleosteus. Ab sofort spielen bei der Beurteilung von Effizienz im Kieferapparat die Hebelgesetze eine große Rolle: Zum Zwecke des Festhaltens von Beute muss der Unterkiefer an den Oberkiefer/Schädel herangezogen werden, was aus der ursprünglich für die Kiemenatmung tätigen Muskulatur besonders eine Muskelgruppe zu einer besonderen Bedeutung brachte: die sogenannten Adduktoren, die nun die Kieferschließmuskulatur bilden. Entsprechend den Hebelgesetzen fungiert das Kiefergelenk hierbei als Angelpunkt (Fulcrum), die davor ansitzende Muskelstrecke ist der Kraftarm und die davor bis zur Kieferspitze ziehende Strecke entspricht dem Lastarm oder der Last. Es ist gerade in einem so frühen Stadium der Kieferentwicklung ersichtlich, dass die genannten Streckenlängen und der genaue Insertionspunkt der Ad- 
duktoren eine große Rolle für die Effizienz spielen, denn zunächst muss ein kleiner, weit hinten (= langer Lastarm!) sitzender Adduktor eine große Masse bewegen, da die beiden Unterkieferäste auch ihr Eigengewicht haben.

Zunächst spielt dies jedoch nur eine eingeschränkte Rolle, da bei den frühen kiefertragenden Fischen die Kiefer gemäß ihrer Herkunft aus dem Kiemenbogensystem nur knorpelig angelegt wurden. Auffällig schnell werden diese Knorpelkiefer jedoch von mehreren Knochen umlagert und dadurch gestützt, was gerade bei den oben genannten, zum Größenwuchs tendierenden Fischen sehr charakteristisch entwickelt war. Nur eine Gruppe allerdings sehr erfolgreicher Fische bildet diese Knochenbedeckung sekundär wieder zurück: die Knorpelfische. Heute weiß man, dass diese Fische nicht besonders ursprünglich sind, sondern dass sie sich von Vorfahren herleiten, die die gerade entwickelte Knochenbedeckung aus schwer rekonstruierbaren Gründen wieder zurückbildeten und zum vollkörperlichen Knorpelskelett zurückkehrten. Der immense Erfolg der Knorpelfische (Haie, Rochen, Chimären) von ihrer Entstehung bis in die heutige Zeit beweist allerdings, dass die Ausbildung von Knochen um die Kiefer herum und auch am restlichen Skelett keine zwingende Bedingung für ein erfolgreiches Leben im aquatischen Bereich ist. Erst beim Landgang macht sich die Ausbildung der festen, kraftübertragenden Knochen zwingend bemerkbar.

Was die „sekundären“ Knorpelfische von den knochenbewehrten Panzerfischen in erster Linie unterscheidet, ist die Ausbildung von Zähnen. Die Panzerfische hatten noch recht unregelmäßige, knöcherne Zusatzplatten auf den Kieferrändern; die Knorpelfische hingegen und sämtliche anderen, weiter entwickelten Fische bilden ab diesem Evolutionsniveau Zähne aus, die prinzipiell wie die zuvor schon geschilderten Schuppen gebaut sind: knöcherne Basis, Dentin-Kern, Enameloid-Kappe, nur dass sie auf den Kieferrändern ansitzen. Unter allmählicher Reduktion der Knochenbasis bildeten die späteren Fische und sämtliche von ihnen abzuleitenden Wirbeltiere zweischichtig gebaute Zähne aus, die nur noch aus dem Dentin und einem Enameloid bestehen. Bei den Schuppen entwickelte sich ein Trend zur Reduktion der Mittelschicht aus Dentin. Dasselbe gilt übrigens auch für die echten Körperschuppen. Entscheidend war die Kombination „feste Kiefer plus Zähne“, wobei „fest" hier nicht mit dem Material Knochen gleichzusetzen ist, wie die Knorpelfischgebisse beweisen. Darüber hinaus sind auf dem Niveau der Knorpelfische noch zwei weitere, auffällige Änderungen zu bemerken: Viele Formen geben die Abplattung des Kopf-/Schulterbereichs auf, um den gesamten Körper stromlinienförmiger auszubilden. Zudem kommt es zur Ausbildung von Flossen, die gelenkig beweglich sind (Brust- und Afterflossen), zu solchen, die die Strömungseigenschaften verbessern (Rückenflossen) und zu großen, kräftigen Schwanzflossen. Diese werden dadurch verstärkt, dass der hinterste Teil der Wirbelsäule entweder in den oberen oder in den unteren Hautlappen der Schwanzflosse zieht, also rückenwärts (dorsal) bzw. bauchwärts (ventral) abknickt. Das Ergebnis dieser Veränderungen ist, dass der gesamte Körper durch die knorpelige Wirbelsäule zu einer Lateralundulation befähigt ist, die wiederum im hinteren Bereich massiv vom großflächigen Schlag der Schwanzflosse unterstützt 
wird. Dieser Bauplan ermöglicht einen Wechsel respektive einen Übergang vom lateralen Undulieren zum hauptsächlich von der Schwanzflosse ausgehenden Oszillieren, wie es die heutigen Hochgeschwindigkeitsschwimmer unter den Fischen aufweisen (Thunfisch, Schwertfisch, etc.). Die stark reduzierte oder nicht mehr vorhandene Hautpanzerung verringert auch das Gewicht der Tiere, sodass erneut neben der verbesserten, schnelleren Bewegungsfähigkeit auch ein Sprung im potentiellen Größenwachstum festzustellen ist. Bei den Landwirbeltieren bildeten sich sekundär erneut Hautpanzerungen aus, die zum Teil auch in einem Schuppen-System organisiert sind. Diese Osteoderme genannten Schuppen werden zwischen Corium und Epidermis gebildet und liegen entweder einzeln in der Haut, bilden einen sehr engen Kontakt bis hin zur Fusionierung oder sind imbrikat angeordnet, sodass im letzteren Fall Schutz und Beweglichkeit einander nicht ausschließen (Abb. 2).
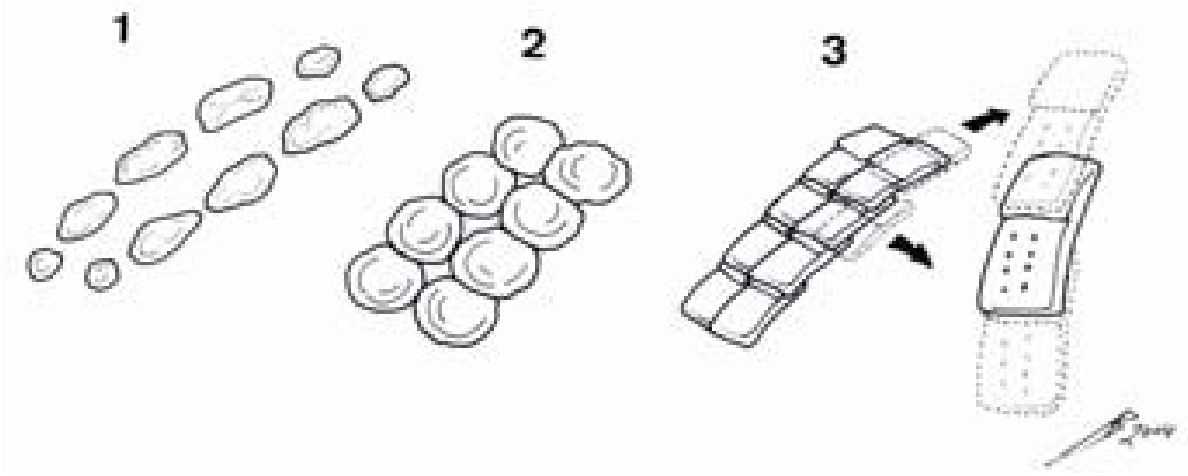

Abb. 2: Knochenschuppen (Osteoderme) der späteren Landwirbeltiere. 2.1: Loser Verband unregelmäßig gebauter Osteoderme; viele paläozoische Amphibien. 2.2: Sehr enger, starrer Verband regelmäßig gebauter Osteoderme; permische Paraiasaurier. 2.3: Sebr enger, imbrikater Verband von Osteodermen, die sich anteroposterior und transversal gegenseitig überschieben können; Detail: Einzel-Osteoderm mit Nachbar-Osteodermen; die verdickte Gleitfläche zeigt nach vorn (rostral). Jurassische bis rezente Lacertilier (Schildechsen: Cordyliformes, Gerrhosauridae).

Die stufenweise optimierte Fähigkeit einzelner Fischgruppen zum schnellen Schwimmen lässt sich erheblich später nochmals auf ähnliche Weise bei den sekundär zum Wasserleben übergegangenen Ichthyosauriern nachvollziehen: Auch sie bilden ähnlich wie die Haie und die Knochenfische zunächst die klassischen, langgezogenen Schwanzflossen aus. Sukzessive treten dann Formen mit immer stärker, meist durchgehend nach ventral abgeknickten Schwanzwirbelsäulen auf, die in der Rekonstruktion einen stabilen unteren Schwanzflossenlappen ergeben. Die Weichteilerhaltung in vielen Fossillokalitäten lieferte darüber hinaus den Beweis für die Existenz der oberen Hauptlappen, sodass der Bewegungstyp „Hauptantrieb von hinten/sehr große Schwanzflosse/Oszillation“ für die erdgeschichtlich viel jüngeren Ichthyosaurier klar nachweisbar ist.

Die Knochenfische gehen denselben Weg wie die Knorpelfische, nur eben mittels eines zunehmend verknöchernden Skeletts. Alle diese Gruppen behalten 
grundsätzlich die laterale Undulation bei und variieren lediglich den tatsächlichen Antriebsanteil der Schwanzflossen, sodass alle Bewegungsstadien in Form verschiedener Typen von Knochenfischen ausgebildet sind: undulieren, subundulieren, suboszillieren, oszillieren, je nach Anteil der Beweglichkeit der Wirbelsäule im Verhältnis zur Schwanzflosse. Alle vier Bewegungstypen gehören zu den sogenannten axialen Bewegungen (Körperachse: Wirbelsäule). Die voll undulierende Bewegung ist nur ohne Außenpanzerung bzw. mit extrem kleinen Schuppen in vollem Umfang möglich.

Tatsächlich bietet die Entwicklung gelenkiger Flossen eine weitere Valenz, nämlich auf die Beweglichkeit der Wirbelsäule sukzessive zu verzichten, den Körper eher zu versteifen und damit zu einem Widerlager umzubauen, und die Flossen gegen dieses Widerlager schlagen zu lassen. Auch dieser Bewegungstyp (paraxiales Schwimmen) wird erst durch den zusätzlichen inneren Umbau der Körperachse ermöglicht, deren Wirbel untereinander spezifische Verankerungen ausbilden können, die wiederum die Beweglichkeit limitieren. Zusätzlich bilden auch einige heutige, paraxial schwimmende Fische einen sekundären Außenpanzer (z. B. Kofferfische), gegen den die Brustflossen schlagen können, oder aber besonders ausgefallene Bewegungsformen aus (langsames Schlagen von Dorsal- und Analflosse der Mondfische).

Von besonderer Bedeutung wurde das kraftvolle Bewegen der Flossen bei den sogenannten fleischflossigen Fischen, den Sarcopterygiern - den Vorfahren der Landwirbeltiere. Ihr Flossenskelett enthält diverse kräftige Knochen, deren Aussehen und Anordnung bereits an die Extremitäten der späteren Landwirbeltiere erinnert: ein kräftiges Einzelelement dicht am Körper (proximal), zwei damit gelenkig verbundene Elemente darunter, und schließlich weiter vom Körper weg (distal) viele kleine Elemente. Diese bewegliche Reihe sowohl in der Vorder- als auch der Hinterflosse ermöglichte einen recht massigen Muskulaturansatz. Dass diese Anlage eine grundsätzliche Ausbildung war, die primär gar nichts mit einer bodenbezogenen Lebensweise zu tun gehabt haben muss, zeigt sich an der Existenz desselben Knochenmusters auch auf einer der beiden Rückenflossen (die hintere), die im Gegensatz zu den vier Flossen-Extremitäten der Flanke bzw. Ventralseite natürlich beim Landgang verloren gingen. Die heute noch lebenden Quastenflosser der Gattung Latimeria zeigen allerdings deutlich, dass gerade die Rückenflossen intensiv zur Antriebserzeugung genutzt werden, indem sie langsame, aberkräftige Schläge zu den beiden Seiten hin ausüben. Die Herausbildung von primär vier funktionsfähigen Beinen zeichnet ab diesem Evolutionsniveau die entsprechend benannten Landwirbeltiere aus, die Tetrapoden. Allerdings treten nunmehr ganz andere Probleme auf, wie sie bei der wesentlich stärkeren Wirkung der Schwerkraft an Land charakteristisch sind: Harte Tragesubstanzen wie die Knochen unterliegen erheblichen Belastungen, die den Kategorien Druck, Zug und Scherkräften zugeordnet werden. Es bedeutet, dass die Knochen neben der Schwerkraft und ihrem Eigengewicht als Gliedmaße in der Bewegung auch das Gewicht des Körpers (Thorax) tragen müssen, unter muskelgesteuerten Bewegungsbedingungen Druck und/oder Zug - oft auch beides abwechselnd - aushal- 
ten müssen und zudem durch komplexere Bewegungen auch noch hoch belastende Scherkräfte aushalten müssen. Hierbei schlägt zu Buche, dass das Hydroxylapatit des Knochens aus genau den genannten Gründen nicht allein auftritt, sondern in Kombination mit einer wichtigen, elastischen Komponente, dem Kollagen. Diese Gruppe von Proteinen zeichnet sich durch eine hohe elastische Belastbarkeit aus, sodass die ausgewogene Kombination aus Knochen und Kollagen zu einer nochmals verbesserten Belastbarkeit führt. Unter Bedingungen eines klassischen Last-Verformungs-Versuchs - also wachsender Belastung durch Druck oder Zug - können von allen Materialien Diagramme erstellt werden, welche die spezifische Belastbarkeit in Form einer Kurve ausdrücken. Das dabei entstehende Spannungs-Dehnungs-Diagramm von frischem Knochen (mit Kollagen) unter Zugbelastung zeigt eine recht lange elastische Reaktionsfähigkeit. Erst ab deutlicher Überlast kommt es zu einem ausschließlich plastischen Verhalten, und auch hierbei tritt jedoch über einen längeren Kurvenverlauf noch kein Bruch auf - eine erstaunliche Leistungsfähigkeit des Hartbaumaterials Knochen. Zudem gelten weiterhin einige Grundlagen der Kräfteeinwirkung auf Festkörper. So kann man besonders Oberarm und Oberschenkel der frühen landbewohnenden Amphibien aufgrund ihrer Massivität mit Balken vergleichen, die horizontal auf je zwei Endpunkten aufliegen. Für diese noch sehr massiv gebauten Einzelknochen gilt, dass die obere Schicht unter Bedingungen der Schwerkrafteinwirkung einer kompressiven Belastung, also Druck, ausgesetzt ist, während die untere Schicht einer Zugwirkung unterliegt. Lediglich die genau zentral gelegene Zone, die sogenannte neutrale Achse (bzw. die neutralen Fasern), verhält sich praktisch belastungslos. Die Extremitätenknochen unterliegen auch hier permanent Dauerbelastungen mit einer vermittelnden Übergangszone, die auch außerhalb der ausgeübten Bewegungen von Bedeutung sind. Erdgeschichtlich schnell entwickelten sich daher nicht mehr massiv gebaute Knochen, sondern stufenweise maschig bis hohl gebaute Elemente, je nach der immer noch nötigen Stabilität.

Anders sieht die Entwicklung beispielsweise beim Kiefer aus. Bereits bei den Knochenfischen, wesentlich ausgepägter jedoch bei den landlebenden Wirbeltieren werden die beiden knorpeligen Unterkieferäste zunehmend von einem Kompositum verschiedener Knochen umhüllt, d. h. mehrere Knochen zusammen bilden eine knöcherne Röhre. Die von dieser Röhre gebildeten Querschnitte sind meist rundlich bis längs-oval. Auch dies lässt sich direkt aus der Festkörpermechanik übertragen: Belastungen aus nur einer Achse resultieren im Einsatz von Wellblech-Strukturen bis hin zu T-Trägern, zweiachsige Belastungen meist in der Konstruktion von Vierkantrohren, vielachsige Belastungssyteme jedoch in Röhren mit rundem Querschnitt. Tatsächlich ist das Ergreifen von Beute mit den Kiefern primär eine Aktivität, die zu mehrachsigen Belastungen führt, da sich Beutetiere wehren können und somit Druck (vom eigenen Wirken der Adduktoren), Zug (Beute) und auch Scherkräfte (Beute) teils über mehreren Achsen wirken. Da Balkenkörper mit Biegebelastungen vorwiegend in den Randfasern reagieren (Modell „an den Enden horizontal aufliegender Balken“, oben: Druck, unten: Zug), müs- 
sen besonders die Querschnittflächen dieser Randzonen vergrößert werden; die Mitte kann hierbei ausgespart werden.

Die erhöhte Stabilität des Kiefers zusammen mit der Optimierung der Extremitätenknochen führte auch an Land zu einem erheblichen Größenzunahmepotential.

Die späteren Fleischfresser unter den Säugetieren gingen in Bezug auf den Unterkiefer einen anderen Weg: Durch den Wegfall der „vielen Elemente“ und die Betonung nur eines, nämlich des zahntragenden Knochens (Dentale) und durch die sehr exakte, auf geradlinige Bewegungsführungen limitierte Struktur des Kiefergelenks (scharnierartige Gelenke) traten zunehmend deutlich weniger Scherkräfte auf. Daher finden wir bei heutigen Carnivoren meist einen T-Trägerförmigen Querschnitt der Unterkiefer.
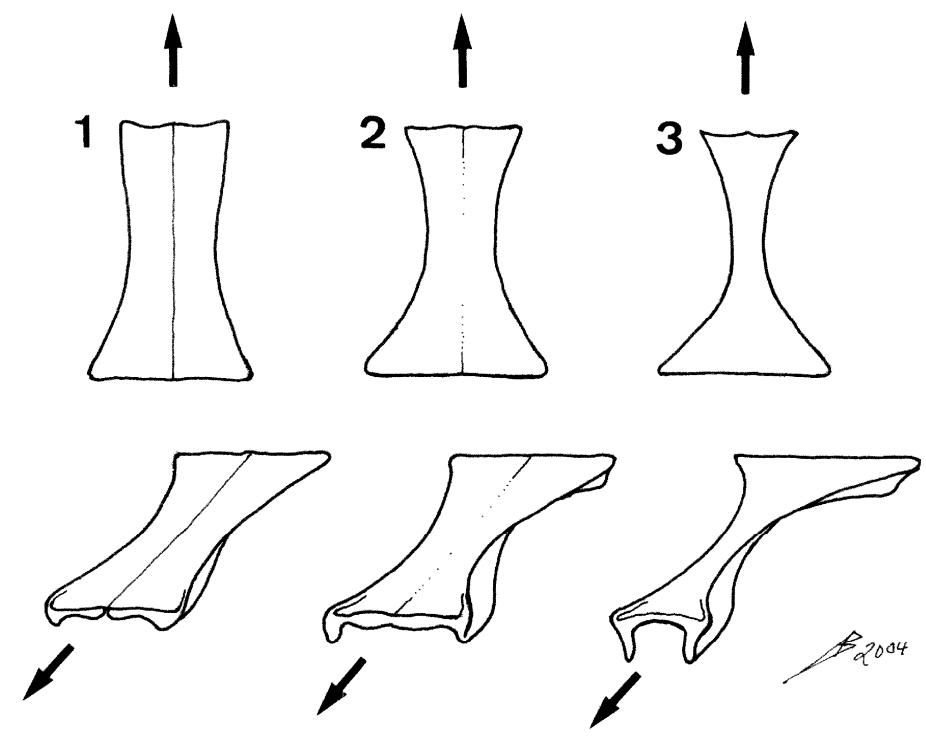

Abb. 3: Stabilisierung der zwischen den Augen gelegenen Frontalia (Schädeldachknochen) bei den Lacertilia (Echsen) am Beispiel dreier, schematisierter Scincomorpha (seit dem Oberen Jura). Die Pfeile weisen jeweils nach vorn, die Einschnürungen links und rechts stellen die Augenhöblen (Orbitae) dar. Oben jeweils die Dorsalansicht von oben, darunter die Ansicht von schräg vorn. 3.1: Ursprüngliche Konfiguration paariger Frontalia bei geringem Einschnürungsgrad (kleine Orbitae). Schwach ausgeprägte Randverdickeungen stabilisieren die beiden Knochen, in der Mitte verlaufen jeweils eigenständige Ränder. 3.2: Weiter entwickelte Konfiguration zweier stärker eingeschnürter Frontalia, die im Erwachsenenalter der Tiere (spätontogenetisch) fusionieren. Die Außenränder werden nach dem Wellblech-Prinzip deutlich verstärkt, die Wülste in der Mitte reduziert. 3.3: Sehr stark eingeschnürtes, unpaares Frontale (= sehr große Orbitae). Die seitlichen Fortsätze sind deutlich länger ausgezogen und ziehen weiter nach hinten, die Mittelwülste sind verschwunden. Das nunmehr frühontogenetisch bereits als Einzelknochen auftretende Element ist trotz seiner Grazilität von böchster Stabilität und vermittelt bereits zwischen Wellblech-und T-Träger-Prin₹ip. Leicht schematisiert, nicht maßstäblich. 
Die primäre Fortbewegungsweise an Land folgte noch dem ältesten aller Modi aus dem aquatischen Milieu, nämlich dem lateralen Undulieren. Die bereits bei den Knochenfischen zunehmend verbesserte Verknöcherung der Wirbel führte zu effizienten Rechts-Links-Schlängelbewegungen, die auch bei heutigen Landwirbeltieren noch gut nachzuvollziehen sind, beispielsweise bei den Eidechsen. Hierbei wird die Wirbelsäule in seitliche Krümmungen bzw. Falten gelegt, deren Wirkung durch das zusätzliche Vor- und Rückziehen bzw. Abstoßen der beiden Extremitätenpaare verstärkt wird. Im ursprünglichen Zustand stehen beide Beinpaare dabei direkt seitlich vom Körper ab, was auch das Grundmuster bei den Amphibien und vielen frühen und auch noch heutigen Reptilien war und ist (Eryops, Ophiacodon u. v. m.) und insbesondere die Tierwelt des Karbon und Perm kennzeichnet (Abb. 4, 4.1). Die hohe Gewichts- und Schwerkraftsbelastung sowie die ungünstigen Hebelverhältnisse (extrem lange Lastarme) der seitlich abstehenden Gliedmaßen limitierte allerdings wiederum das Größenwachstum (Ausnahme sind die teiloptimierten heutigen Warane, insbesondere der großwüchsige KomodoWaran). Neben einer Bewegungsverbesserung durch die Entwicklung des synovialen Gelenks ist daher frühzeitig, bereits während des Perm aufkommend, ein Trend zur Reduktion des seitlichen Schlängelns zu verzeichnen. Die Extremitäten werden in den verschiedenen Reptiliengruppen stufenweise weiter unter den Körper verlagert, was gleichermaßen ein passives Tragen der Körperlast wie auch eine effizientere Fortbewegung der Beinpaare hervorrief, deren Kraft nun nicht für das Halten, sondern primär für die eigentliche Kraftübertragung der Fortbewegung genutzt werden konnte (Abb. 4, 4.2).

Auffällig ist dabei, dass der ursprünglich plan dem Boden aufsitzende Fuß (plantigrade Fortbewegung) mit seinen Elementen Fußwurzel mit Ferse - Mittelfußknochen - Zehenglieder - Endzehen von den verschiedensten Landwirbeltiergruppen parallel zur Ausbildung komplexer distaler Sehnenverspannung vom Boden abgehoben wird. Das betrifft insbesondere die hintere Extremität, die den Hauptteil des Bewegungsschubs liefert, jedoch auch die vordere Extremität. Dabei wird die Ferse verlängert, ebenso werden bei schnellen Läufern die Mittelfußknochen erheblich verlängert und meist von fünf zu weniger Elementen reduziert, es bildet sich eine verwindungssteife Kontaktzone zwischen den darüber liegenden beiden Knochen des Unterschenkels (respektive des Unterarms) aus, die untereinander sukzessive immer weniger beweglich werden, und der eigentliche Bodenkontakt wird nur noch von den Zehen aufgenommen (Zehenlaufen: Digitigradie; Abb. 4, 4.3). Der Vorteil resultiert daraus in zweierlei Weise: Zum einen wird das Fußgelenk funktionell Teil des Beines, was bei der genannten Sehnenverspannung und wenig distaler Muskulatur zu einem weiteren elastischen Sprunggelenkelement führt, zum anderen erhöht sich die Stabilität für schnelle, gerichtete (= primär geradlinige) Bewegungsübertragungen. Dieses Muster ist daher sowohl beim Laufbein zweibeiniger Dinosaurier und der Vögel erkennbar als auch bei vielen unterschiedlichen, heutigen Säugetiergruppen - am ausgeprägtesten natürlich bei den Huftieren (Ungulaten), die besonders schnelle Läufer hervorgebracht haben (Abb. 4, 4.4). Zusätzlich kommt bei jenen großwüchsigen 
Formen, die nur noch kleine Füße (Paarhufer) oder nur noch einen einzigen Zeh (Pferde) haben hinzu, dass dadurch die Masseträgheit der distalen Partien trotz des Längenzuwachses herabgesetzt ist. Die von proximal wirkende Kraft der Ober- und Unterschenkelmuskulatur überträgt sich über Sehnen in die distalwärts gelegenen Beinpartien, ohne dass viel Masse bewegt werden muss.
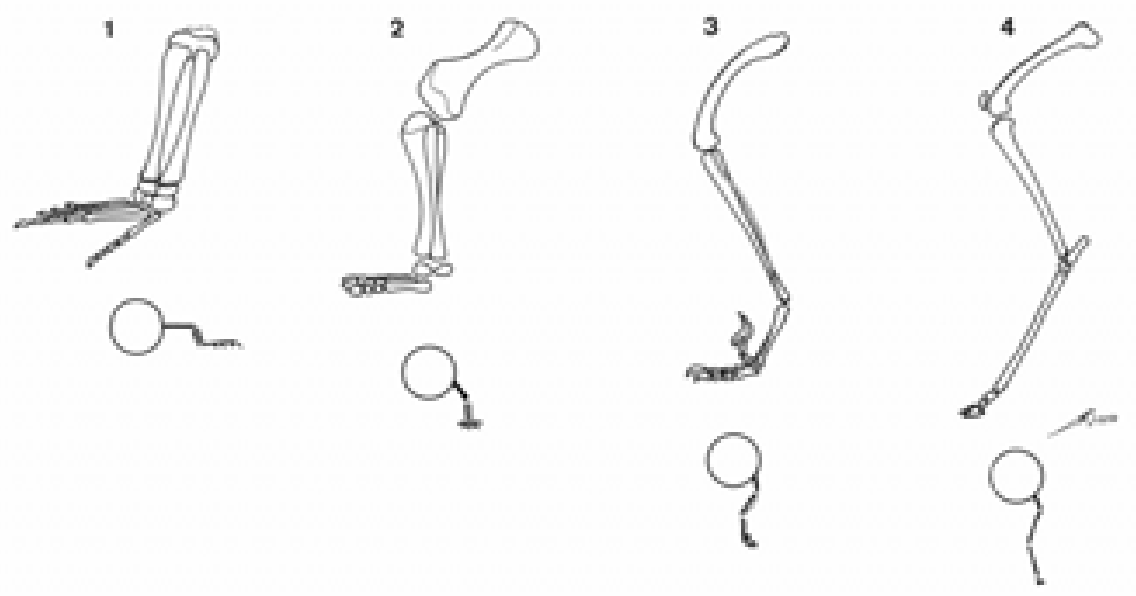

Abb. 4: Der Optimierungsprozess der Hintergliedmaße der Landwirbeltiere. Oben jeweils die Seitenansicht des linken Hinterbeines, darunter eine schematisierte Ansicht von vorn. 4.1: Seitlich abgespreiztes Bein eines schubkriechenden, ursprünglichen Reptils. Ober- und Unterschenkelknochen sind generalisiert gebaut; der Fuß wird von der Seite her abgerollt, weswegen der IV. Zeh der längste ist. Der ganze Fuß sitz̧t dem Boden auf (plantigrad). Petrolacosaurus, Karbon. 4.2: Verbesserte Körperstellung bei einem teilweise aufrecht laufenden, sängetierähnlichen Reptil der Therapsida. Die Fibula (Wadenbein) ist erbeblich dünner als die Tibia (Schienbein), die Zehen sind dabingehend verkürzt, dass der mittlere III. Zeh der längste ist, alle 5 Zehen zeigen in Bewegungsrichtung nach vorn. Der ganze Fuß sitz̧t dem Boden auf (plantigrad). Lystrosaurus, Untere Trias. 4.3: Voll aufrechte, verbesserte Beinstellung eines biped laufenden Dinosauriers (Theropoda) unter dem Körper. Das Femur (Oberschenkelknochen) ist schlank und leicht gebogen, die Tibia lang, die Fibula nur noch spangenförmig ausgebildet, die Metatarsalia (Mittelfußknochen) sind deutlich verlängert und liegen dem Boden nicht auf. Nur die Zehen berübren den Boden = digitigrade Bewegung. Im gezeigten Fall wurde von drei Zehen (II., III. und IV., Zustand der Vögel) zudem einer erhoben getragen, um die große Klaue vor Abnutzung zu schützen. Bambiraptor, Obere Kreide. 4.4: Voll verbesserte Beinstellung eines quadruped laufenden Säugetiers (Mammalia, Artiodactyla). Das Femur ist schlank, die Tibia schlank und lang, aber stabil, die Fibula ist äußerlich nicht mehr erkennbar (mit Tibia fusioniert), die miteinander verschmolzenen verbleibenden zwei Metatarsalia sind extrem verlängert, und nur noch die distal liegenden zwei Zehenspitzen (Hufe) berühren den Boden = unguligrad. Der sebr stark herausragende Fersenknochen (Calcaneus) ist ebenfalls charakteristisch. Neotragus batesi, rezent (Antilope). Zwischen 4.1 und 4.4 wird zunebmend die seitliche Dreh-und Scherfähigkeit des Beins eingeschränkt, was aber zu einer Optimierung der streng nach vorn gerichteten Bewegung führt. 
Von diesem auf Schnelligkeit zielenden Optimierungsprozess weichen lediglich die extremen Giganten ab, also bei den Dinosauriern die Sauropoden, bei den Säugetieren viele ausgestorbene tertiäre Riesenformen und heute die Elefanten. Sie bilden säulenförmige Beinknochen aus, die unter dem Körper liegen, um die Körpermasse passiv zu tragen. Bei ihnen liegen die Füße allerdings meist auch nicht mehr vollständig dem Boden auf, da wahrscheinlich auch die fossilen Formen analog zu den heutigen Elefanten elastische „Faserklumpen“ unterhalb der Fußwurzel eingebaut haben. Dieser „Turnschuh-Fersen-Effekt“ fing die Gewichtsbelastung über die Elastizität wohl besser auf als der rein plantigrade, ursprüngliche Bauplan.

Auf einen ganz besonderen Fall paraxialer Lokomotion, dem Bewegen von befiederten Flügeln gegen einen versteiften und verkürzten Körper bei den Vögeln, kann im Rahmen dieser Kurzpräsentation nicht ausführlich eingegangen werden. Es sei lediglich kurz erwähnt, dass die Knochen der Vögel verständlicherweise sehr leicht sein müssen und daher primär zur Bildung von sehr dünnwandigen Röhrenknochen tendieren. Allerdings sind hier auch Grenzen der Belastbarkeit zu rekonstruieren, d. h. die besonders dünnwandigen Knochen sind von innen her mit einer Vielzahl dünner, querverlaufender Verstrebungen stabilisiert (analog ,Wellblech“-Strategie).

Die Fortbewegung der Säugetiere weist darüber hinaus noch eine weitere Variante auf. Nachdem sich zunächst die Bewegung „optimierte Extremitäten gegen leicht versteiften Körper“ etabliert hatte, weichen die modernen Säugetiere ab Ende der Kreidezeit erstmals komplett vom Grundmuster lateralen Undulierens ab, indem ihre Wirbelsäule sekundär in der Vertikalen beweglich wird eine besonders bei den Walen gut erkennbare Möglichkeit axialen Antriebs. Sowohl bei vielen Pflanzen- als auch bei Fleischfressern (Antilopen, Raubkatzen) kann die Wirbelsäule im unbewegten Grundzustand nach oben gebuckelt sein, was morphologisch dem Schützenbogen und mechanisch einer Vorspannung entspricht. Beim schnellen Rennen dagegen funktioniert dieser Bogen als Beschleunigungskatapult, wenn sich das ganze Tier aus dem resultierenden Spannungszustand heraus in der Bewegung strecken kann. Dies zog natürlich eine komplette Umstrukturierung des Thorax nach sich, die man bei heute lebenden Säugetieren recht gut studieren kann (komplexe Sehnenverspannungssysteme entlang des Rumpfes). Deutlich sichtbar ist dies auch ohne ausgeprägte Grundkenntnisse beim umgekehrten Fall des bereits genannten Bogens, der die nach unten gebogene Halswirbelsäule vieler Pflanzenfresser mit großen, schweren Köpfen über eine zwischen Hinterhaupt und Nacken straff verlaufende Sehnenverspannung trägt.

Bei vielen fortschrittlichen Reptilien, wie den Dinosauriern, aber auch einigen kleinwüchsigen Echsen und natürlich der Vielzahl der Säugetiere fällt die extreme Verbesserung des Hebelsystems der kieferschließenden „Kieferadduktoren“ auf. Hierbei sind die Muskelstränge nicht nur am Schädel selbst wesentlich großflächiger verteilt als bei den ursprünglichen Tetrapoden, sondern sie inserieren am Unterkiefer auch wesentlich weiter vorn und oben, was den Hebel natürlich deut- 
lich verbessert und auch langschnäuzigen Formen einen hohen Kaudruck ermöglich - so besonders gut bei den Krokodilen zu beobachten. Bei den kurzschnäuzigen Formen wird dies durch einen vergrößerten Fortsatz am Unterkiefer erreicht, der nach dem bei den Amphibien und Reptilien dort sitzenden Einzelknochen „Coronoid-Fortsatz“ genannt wird. Bei den Säugetieren wird dieser Fortsatz nur noch vom Dentale gebildet.
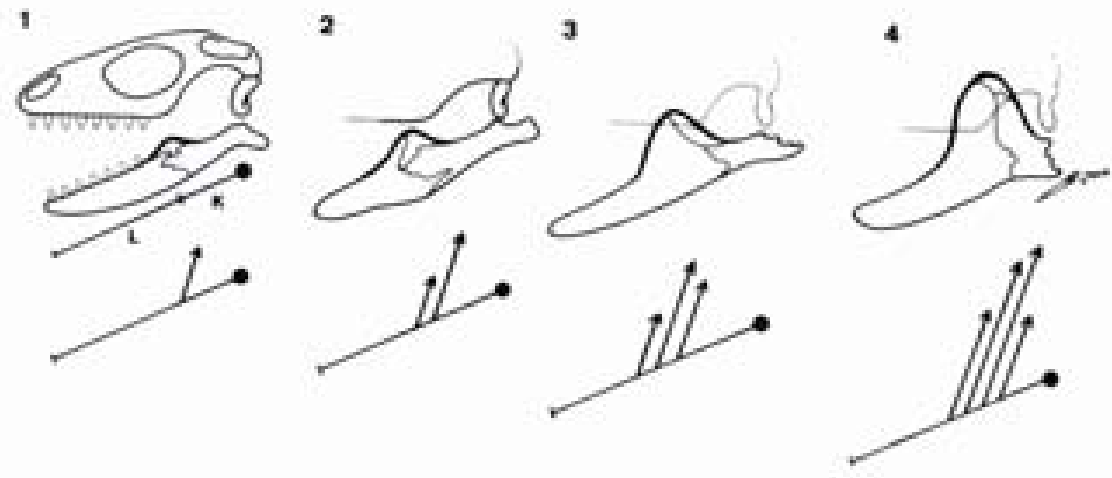

Abb. 5: Die Erböbung des Drucks beim Kieferschließen am Beispiel diverser bein-reduzierter, bodenlebender Lacertilia (alle Beispiele rezent). Oben jeweils die Seitenansicht von links, unten eine stark vereinfachte Visualisierung der Adduktoren-Kräfte. Die Länge der Pfeile stellt die relative Kraft dar, die dann am höchsten ist, wenn die Strecke zwischen der Insertion der Adduktoren (Unterkiefer) und ihrem Ursprung (Schädel: Jochbogen, obere Temporalöffnung, Seitenwand des Neurocraniums) kurz ist und der Ansatz am Unterkiefer weit vorn-oben. Schwarzer Kreis: Angelpunkt (Fulcrum), hier das Kiefergelenk. Die Knochen-Suturen des Dentale (zahntragender Knochen; vorn) und des Coronoids (dunkler hervorgehoben) sind mit Linien dargestellt. 5.1: Generalisierter $V$ ertreter der Scincomorpha, zum besseren Verständnis mit dem korrellierenden, streptostyl gebauten Schädel der Lacertilia. L = Lastarm, K = Kraftarm. Die äußeren Adduktoren haben nur einen geringfügig erhöhten Coronoid-Fortsatz, zum Ansetzen. Sie lassen sich daher als einzelner Pfeil symbolisieren, der eine verbältnismäßig schwache Kaukraft darstellt. Chamaesaura, Cordylidae. 5.2: Leicht verbesserte Situation: Sowohl das Dentale als auch das Coronoid bilden zusammen eine deutlich Erhöhung und Verbreiterung des Adduktoren-Areals. Es resultieren zwei symbolische KraftPfeile, die bereits auf kürzerer Strecke zum Schädel qiehen. Der Kraftarm liegt damit günstiger als bei dem Cordyliden. Acontias, Scincidae. 5.3: Erheblich erhöbter Coronoid-Fortsatz, an dem das Dentale den größeren Anteil hat. Die postdentalen, anderen Unterkieferelemente werden nach hinten verdrängt. Die ansitzende Muskelmasse sitzt dicht am Schädel und kann noch flächiger und noch weiter vorn angreifen, was eine deutliche Verbesserung des Kraftarms gegenüber dem Lastarm darstellt. Drei Kraft-Pfeile resultieren. Dibamus, Dibamidae. 5.4: Der extremste rezente Vertreter der Bodenwübler zeigt einen enorm vergrößerten Coronoid-Fortsat», der in Analogie zu den Sängetieren größstenteils vom Dentale gebildet wird. Die Ansatafläche der Adduktoren repräsentiert etwa die Hälfte der Kieferlänge, die Nähe zum Schädel ist optimal; vier Kraft-Pfeile symbolisieren diesen Vorteil. Trogonoophis, Amphisbaenia. 


\section{Literatur}

Borsuk-Bialynicka, M. (1985): Comments on the kinetics of the lizard skull Fortschritte der Zoologie, Band 30; Duncker/Fleischer (Eds.), Vertebrate Morphology; Gustav Fischer Verlag, Stuttgart, New York, 135-137

Broschinski, A. \& Sigogneau-Russell, D. (1996): Remarkable lizard remains from the Lower Cretaceous of Anoual (Morocco); Annales de Paléontologie (Vert.-Invert.), 82(3), 147-175, 13 Abb.

Dickinson, M. H., Farley, C. T., Full, R. J., Koehl, M. A. R., Kram, R. \& Lehman, S. (2000): How Animals Move: An Integrative View; Science, 288, 100-106

Donley, J., Sepulveda, C. A., Konstantinidis, P., Gemballa, S. \& Shadwick, R. (2004): Convergent evolution in mechanical design of lamnid sharks and tunas, Nature, 429, 61-65

Gans, C. (1974): Biomechanics, J. B. Lippincott Company, Philadelphia, Toronto, $261 \mathrm{pp}$

Hildebrand, M. \& Goslow, G. (2001): Analysis of Vertebrate Structure (Fifth Edition), John Wiley \& Sons, Inc., New York, Chichester, Brisbane, Toronto, Singapore, $635 \mathrm{pp}$

Hutchinson, J. R. (2003): Early birds surmount steep slopes, Nature, 426, 777-778

McGowan, C. (1999): A Practical Guide to Vertebrate Mechanics, Cambridge University Press, Cambridge, $301 \mathrm{pp}$

Reif, W.-E., Hrsg. (1981): Funktionsmorphologie - Paläontologische Kursbücher, Band 1, Paläontologische Gesellschaft Selbstverlag, München, 260 pp

Seilacher, A. et al. (1985): Sonderforschungsbereich 230, Natürliche Konstruktionen - Leichtbau in Architektur und und Natur: Konstruktionsprinzipien lebender und ausgestorbener Reptilien (Principles of construction in fossil and recent reptiles) - Konzepte SFB 230, Universität Stuttgart/Universität Tübingen, Stuttgart, Heft 4, 211 S. 



\title{
Die Sintflut aus geologischer Sicht
}

\author{
Klaus Weber
}

\section{Die Sintflut-Legende}

Die Sintflut-Legende ist Teil der alttestamentarischen Schöpfungsgeschichte, der Genesis, die zwischen 1000 und 500 vor unserer Zeitrechnung, also vor 3000 bis 2500 Jahren, niedergeschrieben wurde. Die hebräische Sintflut-Legende hat jedoch einen sehr viel älteren, sumerischen Vorläufer als Bestandteil des Gilgamesch-Epos. König Gilgamesch regierte während der Ersten Dynastie vor zirka 5000 Jahren im Zweistromland Mesopotamien und gilt als Erbauer der ca. $10 \mathrm{~km}$ langen Mauer von Uruk, die mit 900 Wehrtürmen besetzt war. Er gilt auch als ein sumerischer Herkules und Bezwinger von wilden Tieren und Ungeheuern. Gilgamesch war auch bei den Babyloniern und Assyrern, bei den Churritern und Hethitern ein bekannter Held. In der Babylonischen Fassung zählt das Epos 3500 Textzeilen, von denen etwas 2000 in ihrem Sinn gesichert sind (Baumann, 1972).

Die Sintflut-Legende wurde von George Smith auf sumerischen Schrifttafeln entdeckt, die von Layard und Rassan in der Mitte des 19. Jahrhunderts am Hügel von Kunjundschik bei Mossul am Tigris entdeckt und in großen Mengen, in Körben und Kisten zusammengeworfen, nach London gebracht worden waren.

George Smith, ein junger Kupferstecher und Banknotengraveur, war in den fünfziger Jahren des 19. Jahrhunderts am Britischen Museum angestellt worden, um die Schrifttafeln sortieren und zusammensetzen zu helfen. Er erwies sich als außerordentlich talentiert in dieser Puzzle-Arbeit, und es gelang ihm darüber 
hinaus in sehr kurzer Zeit, die Keilschriftzeichen zu lesen, was ihm das Zusammenfügen von Fragmenten sehr erleichterte.

Die ersten Grundlagen für die Entzifferung der Keilschrift waren von dem Göttinger Lehrer Georg Grotefend gelegt worden, der, wie Hans Baumann (1972) beschreibt, ,als junger Lehrer in Göttingen einige alte Sprachen beherrschte und alle ihm erreichbaren Texte aus Vorderasien studierte. So sehr war er von dem Wunsch besessen, in sie einzudringen, dass er eine Wette abschloss, er werde die Keilschrift entziffern. Das Unwahrscheinliche gelang: Mit einem Scharfsinn ohnegleichen erspürte Grotefend aus einer altpersischen Inschrift einige Königsnamen und erkannte auch das Zeichen, das die Worte trennte." Damit waren die Grundlagen für die Erforschung der Keilschriftzeichen in der ersten Hälfte des 19. Jahrhunderts gelegt.

George Smith war also in der Lage, die Keilschrift der Schrifttafeln zu lesen, und eines Tages entdeckte er die Geschichte von der großen Flut mit dem Rat Ea's, dem Gott der Weisheit, an Utnapischtim (Uitnapistim), König von Surippak (Schurupak), ein Boot zu bauen, um sich und die Seinen, sein Vieh und auch Wildtiere vor einer gewaltigen Überschwemmung zu retten. Viele Details dieser Sage sind identisch mit der Sintflut-Legende aus der Genesis, mit dem Unterschied, dass die spätere Genesis von Autoren verfasst wurde, die mit der Seefahrt offensichtlich nicht vertraut waren. Während Utnapischtim ein Boot baute, dessen Führung er einem Steuermann überließ, zimmerte Noah eine Arche, also in der ursprünglichen Bedeutung des Wortes eine Kiste, in der alles, was er hineinbrachte, vor der Flut gerettet werden sollte.

George Smith stellte seine Entdeckung am 03.12.1872 in einem Vortrag in London der Öffentlichkeit vor. Die Nachricht war eine Sensation. Allerdings war der Text nicht vollständig. Ein Stück der Schrifttafel war abgebrochen und fehlte. Der „Daily Telegraph“ finanzierte eine Expedition unter der Leitung von George Smith, das fehlende Stück des Sintflut-Berichtes zu finden, was ihm im Jahre 1873 tatsächlich auch gelang.

Sehen wir einmal von den religiösen Hintergründen und Ausschmückungen der Sintflut-Legende im Gilgamesch-Epos und in der Genesis ab, und fragen nach einem möglichen realen Ereignis einer so großen Überschwemmung im Zweistromland oder anderswo auf der Welt, die in das Bewusstsein der Menschen eingegangen ist, zu einer Zeit, als es noch keine schriftliche Überlieferung gab, die sozialen Strukturen aber weit genug entwickelt waren, und Gemeinschaften existierten, welche die Erhaltung und Weitergabe von Sagen und Legenden gewährleisteten. Dies begann erst mit der spätsteinzeitlichen Sesshaftwerdung der Menschen und dem Beginn von Ackerbau und Viehzucht. Dieses Entwicklungsstadium ist weltweit zu sehr verschiedenen Zeiten eingetreten. Der „Alte Orient“ mit dem Zweistromland war nach heutigem Wissen jenes Gebiet, in dem diese Entwicklung am frühesten eingesetzt hat. Das war lange vor der sumerischen Erfindung der Keilschrift vor ca. 5000 Jahren und begann hier am Ende der letzten Eiszeit vor ca. 10000 Jahren mit dem Sesshaftwerden der Menschen, mit Ackerbau, Viehzucht und zunehmender Arbeitsteilung in einer klimatisch 
günstigen Region. Diese klimatisch günstige Region Vorderasiens, die auch als der „Fruchtbare Halbmond“ bekannt ist, reicht von der Küste des östlichen Mittelmeeres in einem Bogen über Nordsyrien und die südliche Türkei bis in die Stromoase von Euphrat und Tigris. Hier vollzog sich vor ca. 9000 Jahren erstmalig der Übergang von der Jäger- und Sammlerkultur in eine spätsteinzeitliche, produzierende Ackerbau- und Viehzuchtgesellschaft, der auch als ,neolithische Revolution" bezeichnet wird (Hrouda, 1990).

Die Sintflut-Legende berichtet von einer Naturkatastrophe. Wir würden heute auch von einem Hazard sprechen, wenn es sich um Überschwemmungen, Erdbeben oder Vulkanausbrüche oder um eine Kombination von diesen handelt. Diese Hazards waren für die Menschen des Altertums und bis fast in die Neuzeit Strafen der Götter, weil es keine plausible Erklärung für ihre Ursachen gab.

Vor dem Hintergrund der Sintflut-Legende und ihrer Deutung aus geologischer Sicht möchte ich einige wichtige Prozesse beschreiben, welche Naturkatastrophen vergleichbaren Ausmaßes verursachen können. Ich möchte natürlich im Vornherein sagen, dass dabei eine weltweite Überschwemmung bis zu den Gipfeln der höchsten Berge, wie in der Genesis beschrieben, nicht zur Diskussion stehen kann.

Ich werde diese Prozesse nach ihrer Geschwindigkeit ordnen und mit den sehr langzeitigen Prozessen beginnen: isostatische Prozesse, eustatische Prozesse, Erdbeben und Tsunamis sowie Impakte.

Überschwemmungen sind zeitlich und räumlich begrenzte Hochwasserstände. Es sind reversible Prozesse, für die wir Tage und Wochen, in seltenen Fällen auch Monate ansetzen können. Ist die Überschwemmung ein bleibendes Ereignis, so sprechen die Geologen von einer Transgression, wobei es sich in der Regel um die Ausweitung eines Meeres handelt. Zieht sich umgekehrt das Meer vom Festland zurück, so sprechen wir von einer Regression. Transgressionen und Regressionen sind langzeitige geologische Prozesse, die in Jahrtausenden bis Jahrmillionen ablaufen und zum grundlegenden Inventar der erdgeschichtlichen Entwicklung gehören. Ohne sie gäbe es keine Salz-, Kohlen- und Kohlenwasserstofflagerstätten, und auch viele andere Mineral- und Erzlagerstätten hätten sich nicht bilden können. Die beiden wichtigsten Ursachen sind Hebungen, bzw. Einsenkungen der Erdkruste und Veränderungen im Wasservolumen der Ozeane und Meere.

\section{Isostatische Prozesse}

Veränderungen der Höhenlage der Erdkruste in bezug auf den Meeresspiegel sind in der Isostasie begründet. Diese basiert auf dem Archimedischen Prinzip, wonach z. B. unterschiedlich schwer beladene Schiffe verschieden tief in eine Wasseroberfläche eintauchen. Umgekehrt ragen Körper gleicher Dichte umso höher aus dem Wasser, je dicker sie sind. 


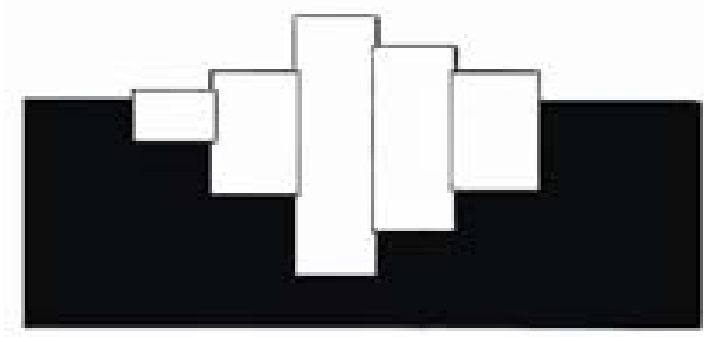

Abb. 1: Isostasie nach Airy. Auftrieb von Körpern gleicher Dichte und verschiedener Mächtigkeit.
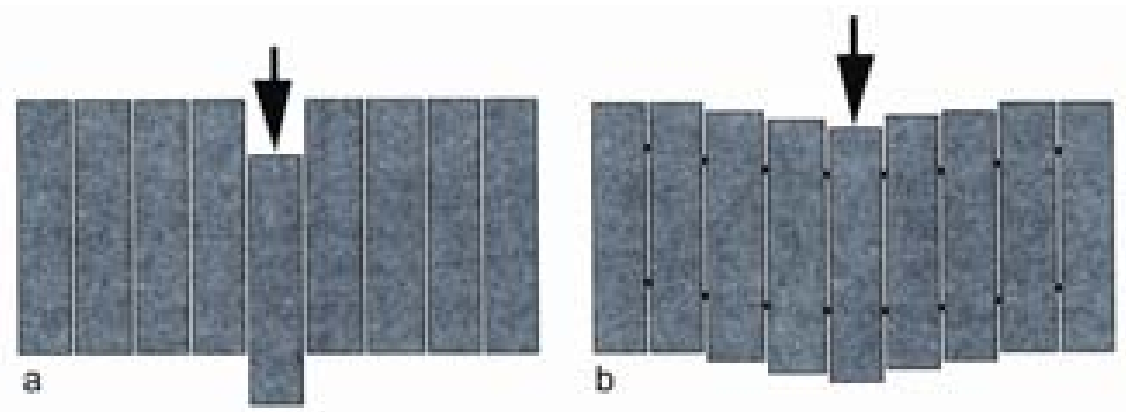

Abb. 2: a) Hydrostatische Isostasie: Auftrieb separater Körper. b) Flexurelle Isostasie: Auftrieb mechanisch gekoppelter Körper.

Die Isostasie ist die Anwendung des Archimedischen Prinzips auf die Lithosphäre. Diese verhält sich nicht wie separate Schiffe im Wasser, sondern die Umgebung belasteter Teile der Lithosphäre wird mit abgesenkt, weil die Lithosphärenplatten ein mechanisches Kontinuum bilden. Ein belasteter Teil der Lithosphäre ist also mit seiner Umgebung mechanisch gekoppelt, wodurch eine flexurartige Einsenkung entsteht. Um diese Anwendung geodynamisch besser zu verstehen, muss etwas über den Aufbau der Erde und die physikalischen Eigenschaften von Gesteinen gesagt werden. Darüber hinaus muss man wissen, dass die Dynamik der Erde, also das Verschieben, Heben und Senken der Lithosphärenplatten, das Öffnen und Schließen von Ozeanen, das Auftürmen von Gebirgen, Erdbeben und der Vulkanismus vom Wärmeüberschuss der Erde angetrieben werden. Ist diese Wärme verbraucht, wird die Erde sterben. Alle Höhenunterschiede werden ausgeglichen, und die Erdoberfläche wird gleichmäßig von Wasser bedeckt sein.

Die Erde besitzt einen Schalenaufbau. Die äußere, relativ starre Hülle wird Lithosphäre genannt. Sie ist zwischen 0 und maximal ca. $300 \mathrm{~km}$, im Mittel $125 \mathrm{~km}$ dick und schwimmt auf einer geringer viskosen Schicht, die wir die Asthenosphäre nennen. Auf dieser Asthenosphäre verschieben sich die Lithosphärenplatten, und in ihr vollziehen sich die isostatischen Ausgleichsbewegungen. 
Die Lithosphäre kann man physikalisch und nach ihrer mineralogischen Zusammensetzung in mehrere Schichten unterteilen, von denen für unsere Betrachtungen eine Unterteilung in den lithosphärischen Erdmantel und die Erdkruste wichtig sind. Die Erdkruste besteht aus relativ leichten Gesteinen mit einer mittleren Dichte von 2,78 g/ccm, die sich im Verlaufe der 4,5 Milliarden Jahre langen Erdgeschichte aus dem Erdmantel abgesondert haben. Der lithosphärische Mantel besteht aus schweren Gesteinen des Erdmantels mit einer Dichte von 3,3 g/ccm. Die fließfähige Schicht, die Asthenosphäre, unterscheidet sich chemisch und mineralogisch nicht vom umgebenden festeren Erdmantel. Ihre Fließfähigkeit resultiert daraus, dass die mit zunehmendem Druck ansteigende Festigkeit der Gesteine von der entfestigenden Wirkung der Temperaturzunahme überholt wird. In größerer Tiefe kehrt sich das Verhältnis wieder um.

Die Eintauchtiefe der Lithosphäre in die Asthenosphäre ist von der mittleren Dichte der Lithosphäre abhängig. Diese wird bei konstanter Dichte der Kruste und des Lithosphärischen Mantels von deren Mächtigkeitsverhältnis bestimmt. Nimmt die Mächtigkeit des Lithosphärischen Mantels (LM) ab, z. B. dadurch, dass aus dem tieferen Erdmantel Wärme zugeführt und damit LM in Asthenosphäre (mit geringer Dichte) umgewandelt wird, so steigt eine Landoberfläche auf. Den Aufstieg über den Meeresspiegel registrieren wir als Regression. Kühlt sich die Lithosphäre umgekehrt $\mathrm{ab}$, weil eine Wärmequelle im Erdmantel versiegt, so nimmt die Dicke des LM und damit auch die mittlere Dichte der Lithosphäre zu und ihre Oberfläche sinkt ab. Ihre Absenkung unter das Niveau des Meeresspiegels registrieren wir als Transgression.

Wo die leichte Kruste relativ zum schweren lithosphärischen Mantel besonders mächtig ist, wie z. B. in den Alpen, dem Himalaya oder den Anden, dort steigt die Erdoberfläche besonders hoch auf. Wo der lithosphärische Mantel relativ zur Kruste sehr mächtig ist, wie z. B. unter den tiefen ozeanischen Meeresbecken, dort sinkt die Erdoberfläche besonders tief ab.

Lokale Wärmezufuhr aus dem Erdmantel verringert die Dicke des lithosphärischen Mantels und damit auch die mittlere Dichte der Lithosphäre und lässt diese aufsteigen. Klassische Beispiele sind stationäre Wärmequellen in Form von Hotspots. Ein solcher findet sich unter Hawaii. Die Verschiebung der westpazifischen Lithosphäre über diesen Hotspot hinweg lässt sich an der Aneinanderreihung submariner Vulkaninseln erkennen, die bis an den Aleutenbogen zu verfolgen sind, wo sie gegenwärtig subduziert wird, d. h. in den tieferen Erdmantel absinkt. Die meisten dieser Inseln haben bei ihrer Anlage über die Meeresoberfläche gereicht. Als sie den Hotspot, über dem sie entstanden sind, verlassen hatten, ist die unterlagernde Lithosphäre wieder abgekühlt und isostatisch abgesunken. Bewohner solcher Inseln hätten dies in ihren Sagen oder Legenden vielleicht als Sintflut registriert.

Die isostatischen Hebungs- und Absenkungsprozesse vollziehen sich sehr langsam, gemessen an unserem menschlichen Zeitmaß. Ein Schiff senkt oder hebt sich in dem Maße, wie es entladen oder beladen wird. Ganz anders bei der Lithosphäre und den geodynamisch, d. h. durch thermische Anomalien und Verfor- 
mungsprozesse verursachten Änderungen der Höhenlage der Lithosphäre, die meist nur Bruchteile von Millimetern pro Jahr betragen.

Die Verformungsgeschwindigkeit oder die Verformungsrate ist die Längenänderung pro Zeit: $\left(1_{1}{ }^{-1} 0 / 1_{0}\right) s^{-1}$.

Die geologischen Verformungsraten liegen in der Größenordnung von $10^{-12} \mathrm{~s}^{-1}$ bis $10^{-15} \mathrm{~s}^{-1}$. Wird ein geologischer Körper in einem Jahr $\left(3,1536 \times 10^{7} \mathrm{~s}\right)$ um $20 \%$ verkürzt oder gelängt, so beträgt die Verformungsrate: $0,2 /\left(3,1536 \times 10^{7} \mathrm{~s}\right)=$ $6,3 \times 10^{-9} \mathrm{~s}^{-1}$. Das ist also die Geschwindigkeit, mit der z. B. ein $1 \mathrm{~m}$ langer Stab in einem Jahr auf 0,8 m verkürzt oder auf 1,2 m gelängt würde. Geologische Verformungsraten sind noch 3 bis 6 Größenordnungen geringer. Für die beschriebenen Längenänderungen unseres Stabes würden wir bei einer Verformungsrate von $10^{-12} \mathrm{~s}^{-1} 6300$ Jahre und bei $10^{-15} \mathrm{~s}^{-1}$ 6,3 Mill. Jahre benötigen.

Das sind die Geschwindigkeiten, mit denen die langzeitigen geodynamischen Prozesse ablaufen, Geschwindigkeiten, mit denen sich die Kontinente Millimeter bis wenige Zentimeter pro Jahr verschieben, sich Bruchteile von Millimetern pro Jahr heben oder senken und mit denen die Gebirge wie Alpen und Himalaya in Jahrmillionen aufgebaut und durch das Zerbrechen der Kontinente neue Meeresbecken in Jahrmillionen angelegt werden. Es sind also, gemessen an unserem menschlichen Zeitempfinden, sehr langsam ablaufende Prozesse, welche die wesentliche Gestaltung der Erdoberfläche bewirken und die sicherlich außerhalb des kumulativen Gedächtnisses der Menschheit liegen.

Allerdings besitzt die Lithosphäre auch elastische Eigenschaften, wie die Ausbreitung seismischer Wellen und die damit verbundenen Erdbeben zeigen. Insbesondere in ihrem oberen, kühleren Teil dominieren die elastischen Eigenschaften. Das hat zur Folge, dass Formänderungsprozesse, welche in der tiefen Lithosphäre fließend stattfinden, in der oberen Lithosphäre bruchhaft erfolgen. Dabei wird die Gesamtgeschwindigkeit der Formänderung durch den Teil der Lithosphäre bestimmt, welcher die höchste Festigkeit besitzt.

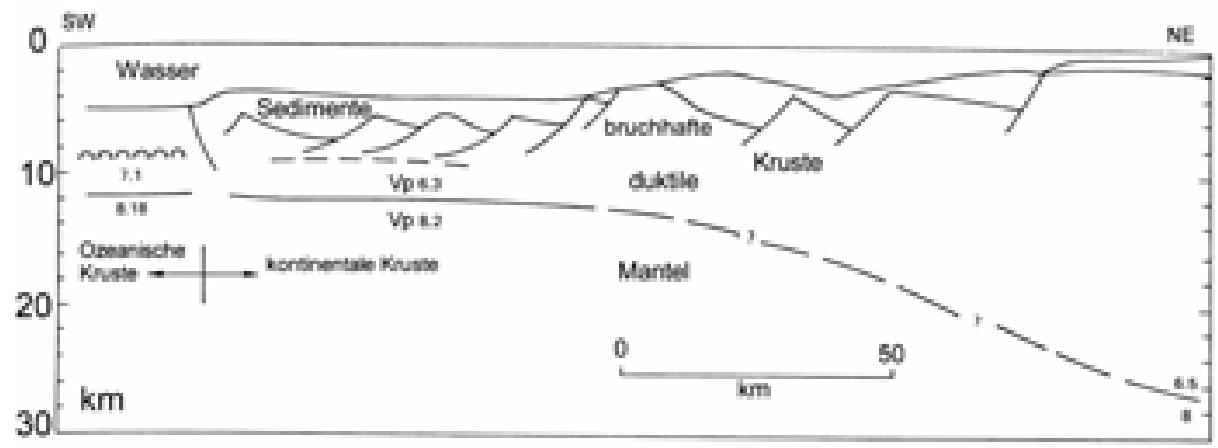

Abb. 3: Struktur des Kontinentalrandes im Bereich der nördlichen Biscaya. Die untere Kruste ist duktil gedehnt worden, während die höhere Kruste bruchhaft gedehnt wurde (nach Montadert et al., 1979). 
Die fließende Verformung der tieferen Lithosphäre führt zum Aufbau elastischer Spannungen in der höheren Lithosphäre, in der Regel in der Erdkruste. Wird die Festigkeit der elastischen Kruste überschritten, entstehen Brüche. Die freigesetzte elastische Verformungsenergie registrieren wir als Erdbeben. So wird also die kontinuierliche Formänderung der fließfähigen (duktilen) Lithosphäre durch zahlreiche diskontinuierliche Formänderungsschritte der bruchhaften Lithosphäre kompensiert.

Beide Verformungsarten können bei gegebenen Drucken und Temperaturen im gleichen Gestein auftreten, je nachdem, ob die Verformung rasch oder langsam stattfindet.

\section{Eustatische Meeresspiegelschwankungen}

Das Abschmelzen von polaren Eiskappen und Hochgebirgsgletschern führt zu einem Anstieg des Meeresspiegels, der sich zwei bis drei Größenordnungen schneller vollzieht, als die isostatischen Prozesse in der Lithosphäre. Für unsere Betrachtungen ist der Zeitraum des Ausklingens der letzten Vereisung und die sich ab $10 \mathrm{ka}$ anschließende und bis heute andauernde Warmzeit, die wir als Holozän bezeichnen, von Interesse. Das ist ein Zeitraum von etwa 15000 Jahren oder $15 \mathrm{ka}$ BP, in dem die Menschen begannen, sesshaft zu werden, Ackerbau und Viehzucht zu betreiben und eine progressive Arbeitsteilung die Entwicklung produzierender sozialer Gemeinschaften begünstigte.

Das Abschmelzen des Inlandeises begann auf der Nordhalbkugel vor ca. 20 ka. Die Erwärmung vollzog sich in zwei Schritten. Einem ersten Schritt globaler Erwärmung zwischen 13 und $12 \mathrm{ka}$, dem eine kurzeitige, einige hundert Jahre andauernde Abkühlung im Mittel vor 11,6 ka folgte, ein Zeitraum, der als die ,Jüngere Dryas“ bezeichnet wird. Danach folgte die holozäne Warmzeit mit einem Maximum des Temperaturanstieges von ca. 9 ka. Vor $6 \mathrm{ka}$ waren die Eiskappen und Hochgebirgsgletscher auf ihr heutiges Niveau abgeschmolzen und der eustatische Meeresspiegelanstieg abgeschlossen. In den mittleren Breiten betrug der Meeresspiegelanstieg im Mittel ca. 110 m. In Mitteleuropa wird dieser Meeresspiegelanstieg auch als die „Flandrische Transgression“ bezeichnet. Zur Zeit des Maximums der letzten Eiszeit war die südliche Nordsee Festland und England war mit dem Kontinent verbunden. Vor $6 \mathrm{ka}$ hatte sich annähernd die heutige Küstenkonfiguration eingestellt.

Während der letzten Eiszeit waren das Gelbe Meer, der Sundaschelf und der Nordaustralische Schelf bis Neuguinea Festland. Mit dem postglazialen Meeresspiegelanstieg mussten sich die Menschen vor der „Asian water front“ in die Kontinente hinein zurückziehen oder besiedelten die verbleibenden Inseln.

Skandinavien war während des Maximums der letzten Eiszeit mit max. 3500 m Inlandeis bedeckt. Unter dieser Last ist die Lithosphäre eingesenkt worden. Seit 8000 Jahren ist das Inlandeis abgeschmolzen, und Skandinavien ist seit dem 
Beginn des Eisrückzuges max. etwa $800 \mathrm{~m}$ aufgestiegen. Das ist ein sehr rascher isostatischer Aufstieg von einigen Millimetern pro Jahr, der wegen der hohen Viskosität der Lithosphäre auch noch nicht abgeschlossen ist und sich mit asymptotisch abnehmender Amplitude noch weitere 20000 Jahre fortsetzen wird. Solche glazio-isostatischen Höhenänderungen sind unterschiedlich verteilt. Sie besitzen dort die größte Geschwindigkeit und Amplitude, wo das Inlandeis besonders dick war, wie in Skandinavien oder in Kanada. Hier übersteigt der glazio-isostatische Aufstieg der Lithosphäre den eustatischen Meeresspiegelanstieg bei weitem, sodass hier das Ende einer Eiszeit mit einem Rückzug des Meeres verbunden ist.

Anders war dies im Bereich der Beringstraße zwischen Alaska und Nordostsibirien. Hier bestand eine Landbrücke zwischen $80 \mathrm{ka}$ und $14 \mathrm{ka}$, die auch während des Maximums der letzten Vereisung über weite Gebiete eisfrei war. Sie bot den Weg für die Einwanderung von Tieren und Menschen von Asien nach Nordamerika. Auf diesem Wege wanderten die Eskimos in Nordamerika ein und die Indianer besiedelten Nord- und Südamerika bis nach Patagonien.

Aralsee und Kaspisches Meer waren während der letzten Vereisung infolge trockenen Klimas Festland und der Spiegel des Schwarzen Meeres war um ca. $150 \mathrm{~m}$ abgesenkt. Das Schwarze Meer war damals ein Süßwassersee, der wie heute von Donau, Dniester, Bug und Don, zeitweise auch von der Wolga durch eine Verbindung zwischen Kaspischem und Asowschem Meer gespeist wurde. Im Verlaufe des postglazialen Anstieges des Meeresspiegels soll vor 7,5 ka das Niveau der Bosporusschwelle, die das bis $1200 \mathrm{~m}$ tiefe Marmarameer vom Schwarzen Meer durch eine $40 \mathrm{~m}$ tiefe Schwelle trennt, überflutet worden sein, wodurch das Schwarze Meer mit dem zweihundertfachen Volumen der Niagara-Fälle innerhalb von etwa drei Jahren um $150 \mathrm{~m}$ aufgefüllt wurde. Ryan und Pitman (1999) vermuten in diesem Ereignis den Ursprung der Sintflut-Legende.

Diese Theorie ist nicht unwidersprochen geblieben, und es gibt eine Reihe gegensätzlicher Beobachtungen (z. B. Aksu et al. 2002). Allerdings deutet eine Erosionsfläche in 123 bis $156 \mathrm{~m}$ Tiefe, die als ehemalige Küstenlinie interpretiert wird (Ryan et al. 1997, Ballard et al. 2001), auf eine Absenkung des Spiegels des Schwarzen Meeres hin. Ob allerdings die Flutung des Schwarzen Meeres ein gradueller Prozess oder ein katastrophales Ereignis war, ist bisher nicht entschieden. Die Umstellung der Süßwasserfauna auf eine marine Fauna nach Ryan et al. (1997) vor ca. 7,5 ka (vor 8,7 ka nach Sperling et al. 2003), kann auch dadurch zustande gekommen sein, dass das allmählich zufließende dichtere Salzwasser das leichtere Süßwasser des Schwarzen Meeres unterschichtet hat. Heute fließt am Bosporus unterhalb $20 \mathrm{~m}$ Wassertiefe höher salinares Wasser aus dem Marmarameer in das Schwarze Meer, während darüber geringer salinares Wasser des Schwarzen Meeres in das Marmarameer fließt. Dieses Gleichgewicht besteht nach Sperling et al. (2003) seit etwa 6,5 ka. 


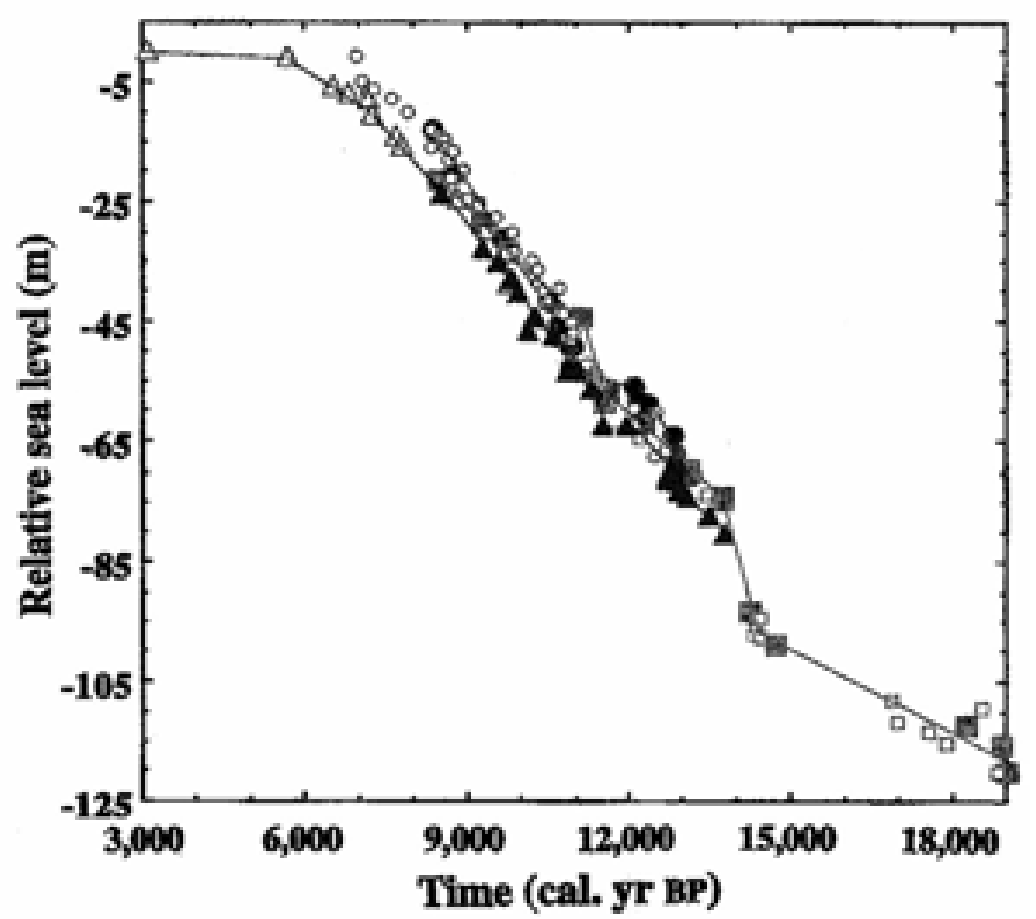

Abb. 4: Postglaziale Meeresspiegel-Kurve niederer Breiten. Seit dem Maximum der letzten Vereisung betrug der Anstieg des Meeresspiegels ca. $120 \mathrm{~m}$. Vor 6 ka war der eustatische Anstieg des Meeresspiegels abgeschlossen (nach Bard et al. 1996).

\section{Seismotektonik und Tsunamis}

Erdbeben entstehen, wenn in der oberen Erdkruste gespeicherte elastische Verformungsenergie die Festigkeit der Gesteine übersteigt und plötzlich freigesetzt wird. Die zerstörende Wirkung von Erdbeben kann gewaltig sein, insbesondere, wenn sie dicht besiedelte Gebiete betrifft, wie wir aus jüngster Zeit von Japan, der Türkei und dem Iran wissen. In historischen und vorhistorischen Zeiten war das nicht anders. Der Untergang von Sodom und Gomorrha war die Folge einer Erdbebenkatastrophe, und die Mauern von Jericho, der ältesten, von Mauern umgebenen Stadt, die wir kennen, sind nicht durch die Posaunen des Josua vor ca. 10000 Jahren eingestürzt, sondern als Folge eines Erdbebens, denn Jericho, $200 \mathrm{~m}$ unter dem Meeresspiegel im Jordantal gelegen, befindet sich in einer seismisch sehr aktiven Zone, auf die auch die Entstehung des Jordangrabens zurückzuführen ist.

Erdbeben sind in der Regel mit Hebungs- und Senkungsprozessen der Erdoberfläche verbunden, die in Form coseismischer Prozesse momentan und in Form postseismischer Prozesse über einen längeren Zeitraum andauernd ein- 
treten. Die verheerende Wirkung von Erdbeben liegt nicht nur in der zerstörenden Wirkung seismischer Erschütterungen, sondern besonders auch in der katastrophalen Wirkung von Tsunamis.

Tsunami ist das japanische Wort für lange Wellen mit Perioden von 3 bis 60 Minuten, die durch mechanische Impulse am Meeresboden angeregt werden. Es kann sich dabei um submarine Rutschungen oder um vulkanische Eruptionen handeln, am häufigsten aber werden Tsunamis durch untermeerische Erdbeben (Seebeben) erzeugt.

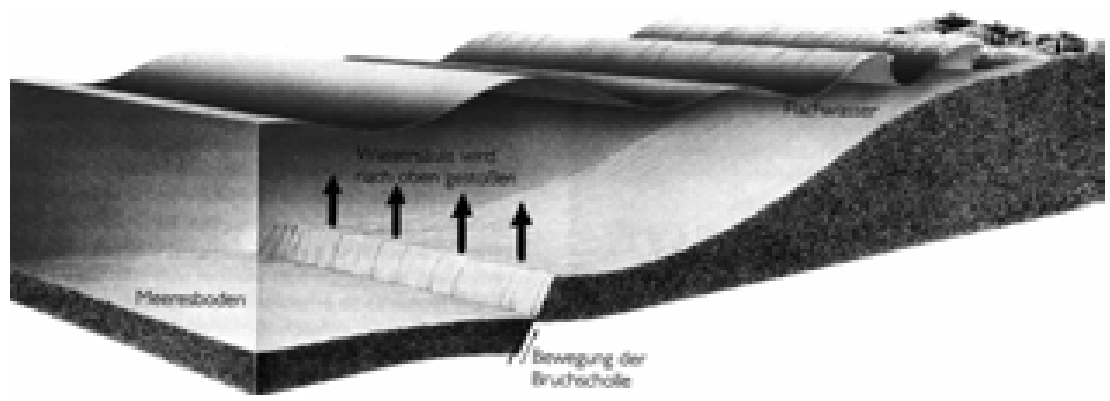

Abb. 5: Tsunami. Die vertikale Verschiebung des Meeresbodens erzengt eine aufrechte Welle über dem Erdbebenzentrum meist mit einer Amplitude von weniger als einem Meter und einer Wellenlänge von mehr als 200 km, die auf hoher See kaum wahrnehmbar ist. Die Welle breitet sich mit einer Geschwindigkeit bis 800 km/Std. aus. Läuft die Welle in flachen Küstengewässern auf, so können Wellen von mehr als 30 m Höhe entstehen, die zu katastrophalen Überflutungen führen (aus Press \& Siever, 2003).

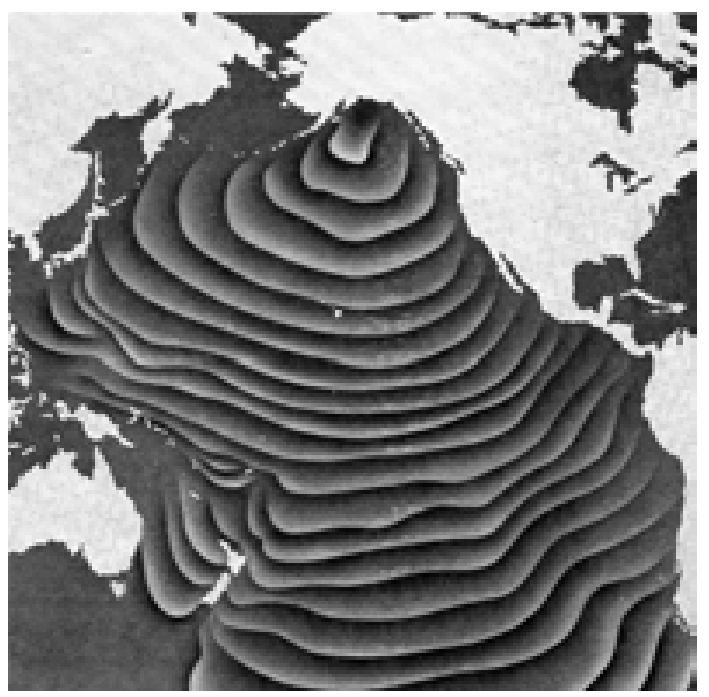

Abb. 6: Ausbreitung eines Tsunami über den Parifischen Ozean mit Ursprung vor der Küste Alaskas. Die Karte zeigt seine stündliche Position als Isolinien an (Der Große Krüger Atlas der Ozeane, W. Krüger Verlag, Frankefurt 1979). 
1960 löste ein Erdbeben vor der Küste Chiles einen Tsunami aus, der den gesamten Pazifik überquerte und in Japan zahlreiche Menschenleben forderte. Der Tsunami, der 1883 durch den Ausbruch des Krakatau in Indonesien ausgelöst wurde, erzeugte eine $40 \mathrm{~m}$ hohe Flutwelle, die 36000 Menschenleben forderte. Am stärksten durch Tsunamis gefährdet sind tiefliegende Küstenregionen, deren gegenwärtiges Gefährdungspotential noch dadurch steigt, dass sie dicht besiedelt sind. Die häufigsten Tsunamis treten im pazifischen Raum auf. Im Atlantik sind sie seltener. Sie können jedoch überall dort auftreten, wo untermeerische Erdbeben, Vulkane oder Rutschungen möglich sind.

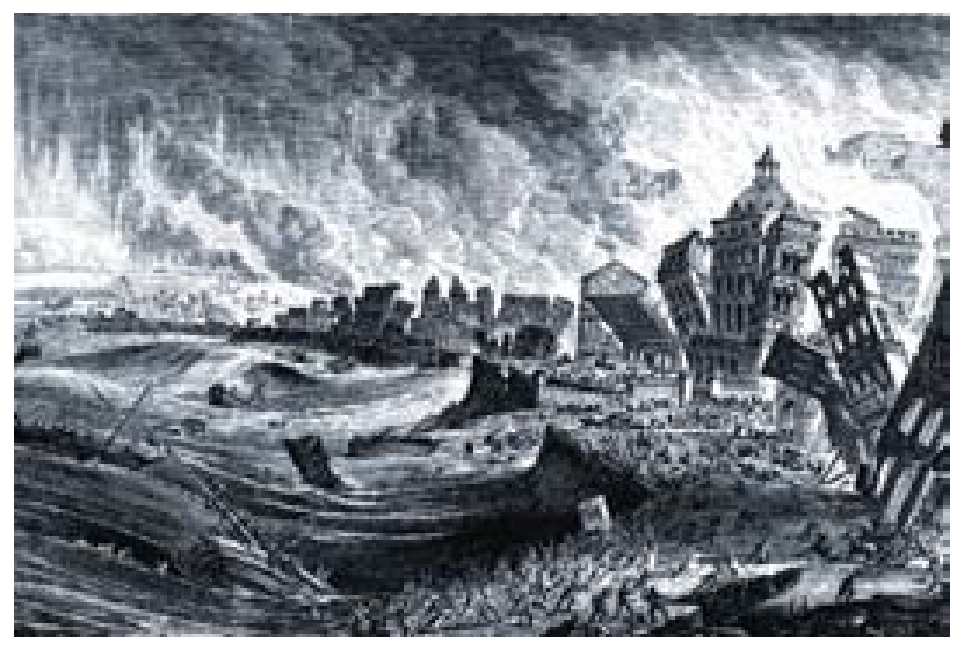

Abb. 7: Dem Erdbeben von Lissabon am 01.11.1755 folgte ein Tsunami, der die Stadt fast völlig zerstörte (Der Große Krüger Atlas der Ozeane, W. Krüger Verlag, Frankfurt 1979).

\section{Impakte}

Impakte, d. h. Einschläge von Meteoriten oder Kometen, können das non plus ultra globaler Katastrophen sein. In der Frühzeit ihrer Entwicklung, d. h. vor mehr als 4 Milliarden Jahren, war die Erde einem massiven Bombardement solcher Himmelskörper ausgesetzt, deren Spuren nahezu ausgelöscht sind. Auf dem Mond sind diese Spuren mangels freiem Wasser und einer Atmosphäre erhalten und zeichnen das Gesicht vom „Mann im Mond“.

Wir kennen ca. 165 Impaktstrukturen auf der Erde mit Durchmessern zwischen $15 \mathrm{~m}$ und $300 \mathrm{~km}$ (Langenhorst 2004). Bekannt ist z. B. das Nördlinger Ries in Süddeutschland, das auf einen Meteoriteneinschlag vor ca. 32 Ma zurückzuführen ist. Je jünger die Impaktstrukturen sind, umso besser sind sie erhalten. So z. B. der 49000 Jahre alte Barringer-Krater in Arizona (Abb. 8b) mit 1,2 km Durchmesser oder der teilweise von Dünen überdeckte Rote Kamm (Abb. 8a) in der Namibwüste Namibias, der ein Alter von 3 Mill. Jahren besitzt. 


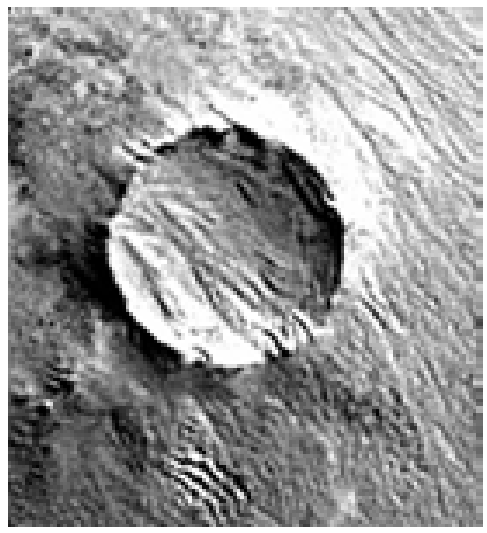

Abb. 8 a: Impakt-Krater Roter Kamm in der Namibwüste, Durchmesser 3 km (Satellitenbildausschnitt TM 5).

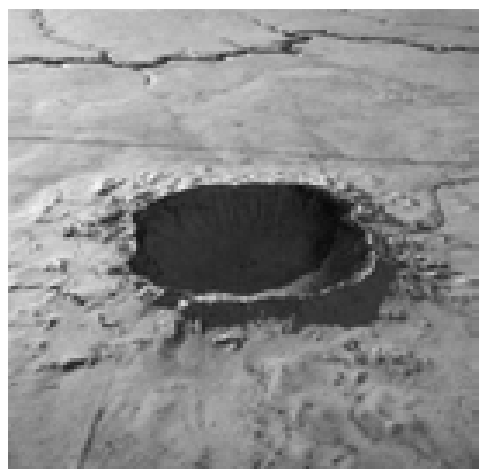

Abb. 8 b: Impakt-Krater von Arizona, Durchmesser 1,2 km.

Impakte können, wenn sie im Meer einschlagen, einen Tsunami erzeugen. Ihre Wirkung auf dem Lande ist eher von begrenzter Auswirkung, wenn es sich um Einschläge, wie beim Arizona-Krater oder beim Roten Kamm handelt. Der Impakt, welcher das Nördlinger Ries in Süddeutschland erzeugte, hatte schon weiterreichende Auswirkungen. Der Meteorit kam von Westen, und der Streufächer seiner Auswürfe (Tektite) reichte bis nach Böhmen.

Um eine Sintflut im biblischen Sinne zu erzeugen, muss ein solcher Einschlag im Meer erfolgen und eine Dimension haben, die weit über der aller bekannten Impakte liegt, ausgenommen dem Impakt von Chicxulub im Bereich der YucatanHalbinsel Mexikos. Dieser Impakt hat vor $65 \mathrm{Ma}$ am Ende der erdgeschichtlichen Kreidezeit stattgefunden. Er wurde durch einen ca. $10 \mathrm{~km}$ großen Asteroiden verursacht und hat einen Krater von $300 \mathrm{~km}$ Durchmesser und einer Tiefe von 17 bis $20 \mathrm{~km}$ erzeugt, wobei Zerstörungen der Lithosphäre bis in Tiefen von 45 bis $60 \mathrm{~km}$ eingetreten sind. 
Heute ist der Krater von jüngeren Sedimenten bedeckt und nur durch Bohrungen und geophysikalische Untersuchungen nachgewiesen. Es handelt sich um eines der größten Impakt-Ereignisse im inneren Sonnensystem nach dem Ende des frühen Bombardements vor 4 Milliarden Jahren und um den größten Impaktkrater, der von der Erde bekannt ist. Es wird vermutet, dass dieser Impakt durch den Auswurf gewaltiger Massen von Gesteinsstaub und Wasser in die Atmosphäre zu einer jahrelangen, globalen Klimakatastrophe geführt hat, in deren Folge zahlreiche Tierarten, u. a. die Saurier, ausgestorben sind.

Edith Kristan-Tollman und Alexander Tollman (1992) haben dieses Ereignis zum Anlass genommen, den Impakt eines Kometen, eines Himmelskörpers aus Gestein und Eis, zu postulieren, der am 23. September vor $9545 \pm$ einige Jahre um 3 Uhr morgens in der nördlichen Hemisphäre eingeschlagen sein soll. Vor dem Auftreffen habe er sich in sieben Teilkörper aufgelöst, die alle ins Meer fielen. Trotz gründlichen Studiums der Arbeiten von Tollmann \& Tollmann haben sich keine überzeugenden Angaben finden lassen, worauf dieses Datum basiert. Das Ereignis soll in seiner Dimension dem am Ende der Kreidezeit vergleichbar gewesen sein.

Wir haben zahlreiche wissenschaftliche Beweise für den Impakt vor $65 \mathrm{Ma}$, jedoch keinen einzigen Beweis für ein vergleichbares Ereignis vor 9500 Jahren, der einer eingehenden wissenschaftlichen Überprüfung standhält, wie die Erwiderung von Deutsch et al. (1994) auf die Veröffentlichungen der Tollmanns zeigt. Natürlich haben die Ansichten der Tollmanns bei Kreationisten und Excegeten des Alten Testaments großen Anklang gefunden. Ein Impaktereignis dieser Dimension von etwa $10 \mathrm{ka}$, d. h. also nach der letzten Eiszeit, aus einer Zeit also, von der wir sehr umfangreiche und detaillierte geologische Kenntnisse besitzen, müsste in den postglazialen Sedimenten und Strukturen eindeutig dokumentiert sein. Deshalb können wir diese Theorie ad acta legen und wollen uns jetzt wissenschaftlich besser fundierten Daten und Ansichten zuwenden, um den geologischen Hintergründen der Sintflut-Legende näher zu kommen.

Wie wir gesehen haben, gibt es viele Ursachen für regionale und lokale Überflutungen. Die Mechanismen wie Isostasie, Eustasie, Erdbeben, Tsunamis und Impakte können isoliert, jedoch auch überlagernd auftreten. Transgressionen, d. h. bleibende Überschwemmungen großen Maßstabes, wie auch Regressionen, also Rückzüge des Meeres über sehr große Gebiete, gehören zum Grundinventar der Erdgeschichte. Bei allen diesen Prozessen ist immer Leben zu Schaden gekommen oder hat davon profitiert, d. h. seit 4 Milliarden Jahren, seitdem es Leben auf der Erde gibt.

Eine Sintflut-Legende jedoch konnte erst entstehen, seitdem es Menschen gibt, die in sozialen Gemeinschaften lebten und sprachlich, später dann auch schriftlich kommunizierten. Damit ist der Zeitraum abgesteckt, in welchem wir nach Ereignissen für den Ursprung der Sintflut-Legende suchen können. Es liegt auf der Hand, dass damit nur der Zeitraum seit dem Ausgang der letzten Eiszeit infrage kommt. Da das Gilgamesch-Epos und damit die Sintflut-Legende ihren Ursprung im Zweistromland von Euphrat und Tigris haben, wollen wir über- 
prüfen, ob wir aus geologischer Sicht einen Ursprung dieser Legende hier finden können.

Mesopotamien und der Persische Golf liegen an der Grenze zweier Lithosphärenplatten, der Arabischen und der Eurasischen Platte (Abb. 9). Die Arabische Platte bewegt sich in nördlicher Richtung unter die Eurasische Platte. Auf ihrer rückwärtigen Seite wird dabei das Rote Meer geöffnet und auf ihrer Frontseite das Zagros-Gebirge zusammengeschoben. Unter der Auflast des Zagros-Gebirges wird der Nordostrand der Arabischen Platte abgesenkt und bildet das Tiefland von Mesopotamien und den Persischen Golf. Die Kollisionszone ist durch häufige Erdbeben gekennzeichnet, was für unsere spätere Interpretation der Sintflut-Legende wichtig sein wird. Der Persische Golf ist ein sehr flaches Meer mit seiner stärksten Absenkung vor dem Zagros-Gebirge. Im überwiegenden Teil ist die Wassertiefe geringer als $60 \mathrm{~m}$. Die Wassertiefe übersteigt nur an einigen Stellen geringfügig die $100 \mathrm{~m}$. Zur Zeit der letzten Eiszeit im Zeitraum bis etwa vor $15 \mathrm{ka}$ war der Persische Golf also trocken und durch Wüstenklima geprägt, wie fossile Dünenfelder am Meeresboden belegen (Sarntheim, 1972). Vor $12 \mathrm{ka}$ war er etwa bis zu 60 m-Tiefenlinie geflutet.

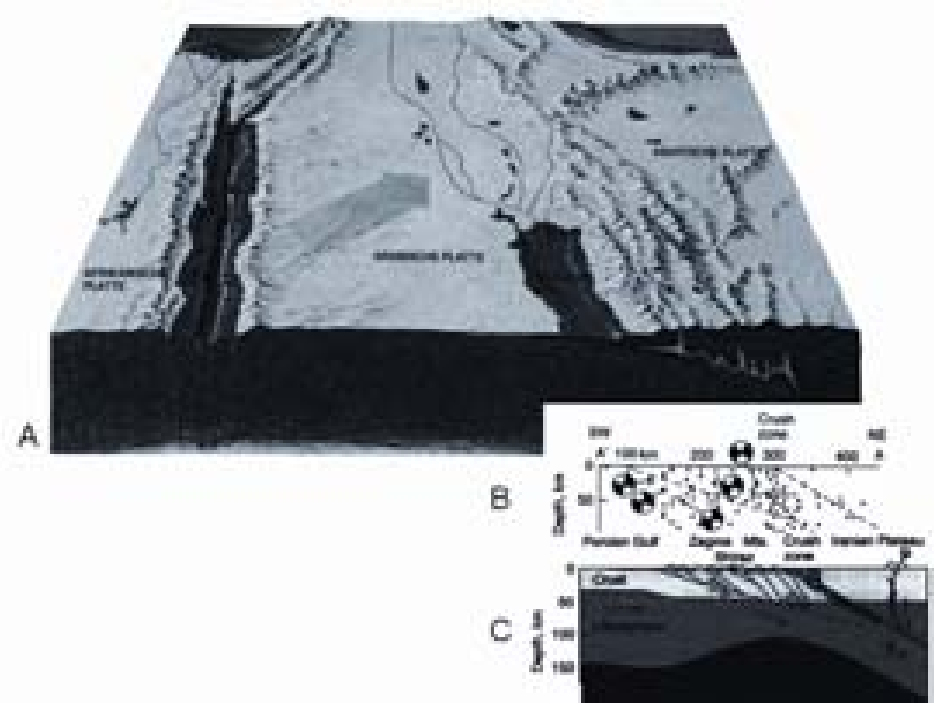

Abb. 9: Abtauchen der Arabischen Platte unter die Eurasische (Asiatische) Platte mit der Zone hoher Seismizität im Persischen Golf und dem Zagros-Gebirge (A Blockbild aus: Der Große Krüger Atlas der Ozeane, W. Krüger Verlag, Frankfurt 1979, B Verteilung der Erdbeben aus: Stöcklin 1974, C geologisches Profil aus: Bird 1978).

In der Darstellung menschlicher Siedlungsgebiete vor 25 bis $15 \mathrm{ka}$ von Madeyska et al. (1992) wird der Persische Golf als inhospitabel und das Gebiet des heutigen Zweistromlandes für die menschliche Besiedlung nur als bedingt geeignet bezeichnet. 
Das änderte sich mit einem Temperaturanstieg im Zeitraum zwischen 13 und 12 ka (Bölling-Warmzeit), dem eine kurze Abkühlungsphase (Jüngere Dryas) von einigen hundert Jahren zwischen 12 und $11 \mathrm{ka}$ folgte. Der nachfolgende Temperaturanstieg erreichte sein Maximum vor $9 \mathrm{ka}$. Bis etwa vor $6 \mathrm{ka}$ empfingen die Sahara und die Arabische Wüste deutlich mehr Niederschläge als heute. Die Arabische Halbinsel trug eine Savannenlandschaft und bot gute Bedingungen für die menschliche Besiedlung. Die zahlreichen steinzeitlichen Artefakte aus der Zeit zwischen 10 und 6 ka, die in der Saudi-Arabischen Wüste Rub-Al Khali zu finden sind, belegen den Klimawandel bis vor etwa 6 ka. Bessere Klimabedingungen herrschten auch nördlich des Persischen Golfes bis nach Pakistan und Indien.

Diese höheren Niederschläge in Afrika und Südasien sind vermutlich auf eine höhere Intensität des Monsuns zurückzuführen. Man vermutet auf Grund paläoklimatischer Daten und allgemeiner Zirkulationsmodelle für den Zeitraum zwischen 12 und $5 \mathrm{ka}$ eine orbital induzierte Zunahme der Sonneneinstrahlung während der Sommermonate und eine entsprechende Abnahme während der Wintermonate auf der Nordhalbkugel. Diese erhöhte den Temperatur-Kontrast zwischen Land und See, was zu einer Intensivierung des Sommermonsuns und damit zu erhöhten Niederschlägen, zu höherem Grundwasserspiegel und zur Auffüllung von Seen in Gebieten führte, die heute arid sind.

In den Sommermonaten entsteht durch die aufsteigende Warmluft ein Tiefdruckgebiet über dem Persischen Golf und dem Indischen Subkontinent. Der Südwestmonsun füllt dieses Tiefdruckgebiet mit reichlichen Niederschlägen auf. Umgekehrt bildet sich in den Wintermonaten ein stabiles Hochdruckgebiet über Asien, vom dem aus durch den Nordostmonsun Tiefdruckgebiete über dem Arabischen Meer und dem Indischen Ozen aufgefüllt werden. So konnten die Vorsumerer und Sumerer in den Sommermonaten mit dem Südwestmonsun über den Persischen Golf nach Indien segeln und in den Wintermonaten mit dem Nordostmonsun zurückkehren.

Wir dürfen aber auch annehmen, dass die höheren Luftdruckgegensätze zur Zeit der Vor- und Frühgeschichte Mesopotamiens zu einem intensiveren Wettergeschehen führten, und dass auch Taifune über das Arabische Meer und den Persischen Golf jagten, wie das auch in der Genesis beschrieben ist. Die SintflutLegende im Gilgamesch-Epos berichtet von einem tagelangen Orkan aus südlicher Richtung, wobei es sich vermutlich um besonders schwere Unwetter im Sommermonsun gehandelt haben dürfte. Durch Starkregen und Rückstau des Meerwassers kann, wie auch in der Gegenwart, das flache Schwemmland vom Persischen Golf her überflutet werden, wobei die niedrig gelegenen Siedlungsgebiete völlig zerstört werden können.

Der postglaziale Meeresspiegelanstieg (Flandrische Transgression) zwischen 9 und $6 \mathrm{ka}$ überlagerte sich mit der holozänen Klimaverbesserung im Vorderen Orient. Durch das geringe Gefälle der flachen Golfsenke schritt die Flandrische Transgression relativ schnell voran. Nach Sartheim (1971) verlagerte sich die Küstenlinie in der Längsachse des Golfes um 100 bis 120 mm/a. Nützel (1975) kommt mit $200 \mathrm{~km}$ in 2000 Jahren zu vergleichbaren Werten. Diese relativ schnell 
voranschreitende Transgression führte zu spürbaren, an manchen Orten und zu manchen Zeiten auch katastrophalen Veränderungen des natürlichen Lebensraumes in den vermutlich relativ dicht besiedelten, küstennahen Flachländern des Golfes.

Wie die Kurve des postglazialen Meeresspiegel-Anstieges (Abb. 4) zeigt, war dieser Anstieg des Meeresspiegels auf das heutige Niveau vor $6 \mathrm{ka}$ im Wesentlichen abgeschlossen. Das trifft auch für den Persischen Golf zu, der damals zum Höchststand der Transgression jedoch noch ca. $400 \mathrm{~km}$ in nordwestlicher Richtung landeinwärts bis etwa vor die Tore des späteren Bagdad reichte. Euphrat und Tigris mündeten zu dieser Zeit noch als getrennte Flüsse in den Persischen Golf. Über die getrennten Mündungsgebiete von Euphrat und Tigris berichtet auch die Sintflut-Legende im Gilgamesch-Epos. Als Utnapischtim, der vorsumerische Noah, die Sintflut überstanden und den Göttern Brandopfer gebracht hatte, wurden er und sein Weib vom Kriegsgott Bel, der die Sintflut angezettelt hatte, den Göttern gleich gemacht und ,in die Ferne, an die Mündung der Ströme entrückt", womit nur Euphrat und Tigris gemeint sein können.

Mit dem Abklingen der Flandrischen Transgression konnte die Aufschüttung ihres gemeinsamen Deltas beginnen. Die geologische Voraussetzung dafür war, wie auch bei anderen holozänen Delta-Aufschüttungen, dass die Rate der fluviatilen Sedimentzufuhr die abnehmende Rate des Meeresspiegel-Anstieges überschritt. Deshalb hat auch das antike Ur vor $5 \mathrm{ka}$ an der Küste des Persischen Golfes gelegen, und Woolley's „Sintflut-Horizont“ bei Ur, eine ca. 2,5 m mächtige tonige Schicht zwischen präsumerischen Grabstätten unten und sumerischen Grabstätten oben (Woolley, 1930), dokumentiert die Auffüllung des EuphratDeltas, die vor ca. 6 ka begann.

Ein wichtiges Ereignis, das vor etwa 6 ka begann, ist die deutliche Klima-Verschlechterung zu trockenerem Klima und Wüstenbildung von der Sahara, über die Arabische Halbinsel bis nach Südasien. Völker mussten ihre bisherigen Siedlungsräume verlassen und in Gebiete einwandern, die ausreichend Nahrung boten. In den Einwanderungsgebieten entstand ein Bevölkerungsdruck, der die kulturelle und politische Entwicklung beeinflusste und schließlich zu Dynastien wie in Ägypten und auch Mesopotamien führte. Zu diesen „Umsiedlern“ gehörten auch die Sumerer, die vor $6 \mathrm{ka}$ vermutlich von Osten in das Zweistromland einwanderten.

Die Sintflut-Legende, auf dem Gilgamesch-Epos basierend, berichtet in religiös übersteigerter und zeitlich verkürzter Form über den postglazialen Meeresspiegelanstieg. Diese Erfahrung haben die holozänen Menschen in allen mittleren und niederen Breiten gemacht. Dementsprechend gibt es in vielen Kulturen ähnliche Sagen und Legenden (Riem, 1925).

Viele Naturereignisse, über die in der Sintflutsage des Gilgamesch-Epos und in der Genesis berichtet wird, haben in der holozänen Geschichte Mesopotamiens stattgefunden. Der progressive Anstieg des Meeresspiegels erhöhte die Seismizität längs der ohnehin schon seimisch aktiven Plattengrenze zwischen Arabischer und Euroasiatischer Platte. Erdbeben und Tsunamis, die das flache Land weiträumig 
überfluteten, waren die Folge. Durch den Aufbau seismischer Spannungen wurden die wassergesättigten Sedimente des Mesopotamischen Schwemmlandes komprimiert, sodass ,sich die Erde auftat“ und infolge des hohen Grundwasserstandes „Wasser aus der Tiefe austrat", eine Erscheinung, die wir aus rezenten Erbebengebieten kennen.

Die erhöhten Luftdruckgegensätze ließen den Sommermonsun in größerer Heftigkeit über das Land hereinbrechen als in heutiger Zeit. Er war vermutlich von Taifunen, die den Himmel verdunkelten und heftigen Gewitterregen begleitet, deren Blitze den Himmel beleuchteten. Zwischen Monsunregen und Erdbeben kann ein ursächlicher Zusammenhang bestehen, dadurch, dass durch starke Luftdruckänderungen Erdbeben ausgelöst werden können. Dies ist aus historischen Zeiten hinreichend bekannt.

Da die Menschen jener Zeit bevorzugt die küstennahen Flachländer des Zweistromlandes besiedelten, haben sie über Generationen den Anstieg des Meeresspiegels erleben müssen. Die präsumerischen Siedler haben ihre Hütten und Felder wegen des ansteigenden Meeresspiegels immer wieder verlagern müssen. Da sie auch vom Fischfang lebten, haben sie Boote besessen und waren vermutlich auch in der Lage, den Sommer- und Wintermonsun nutzend, größere Entfernungen auf dem Meer zurückzulegen. Sie hatten also Boote, in denen sie sich und ihre Habe in Sicherheit bringen konnten, wenn Überflutungen hereinbrachen. Die übersteigerte Legende von der Arche entsprang dem kumulativen Gedächtnis vieler Generationen, welche die gleichen Erfahrungen gemacht hatten.

Die Einbeziehung der Götter entsprach den religiösen Vorstellungen jener Zeit. Nach der sumerischen Mythologie waren die Menschen von den Göttern erschaffen zu dem alleinigen Zweck, ihnen dienstbar zu sein und für ihr Wohlergehen zu arbeiten. Da man die natürlichen Ursachen der Naturkatastrophen nicht kannte, konnten es nur zornige Götter sein, die das Unglück sandten. Der Zorn des Götterkönigs Enlil war entbrannt, weil die Menschen, die sich unterdessen über die Maßen vermehrt hatten, Lärm und Getöse verbreiteten, wodurch Enlil nicht mehr ruhig schlafen konnte. Nachdem er zu ihrer Reduzierung Krankheit, Dürre und Hungersnot geschickt hatte, die der weise Gott Ea (Enki) immer wieder abwenden konnte, bevor die Menschen ausgerottet waren, schickte er schließlich die große Flut. Aber Ea wollte verhindern, dass die Götter die Arbeit der Menschen, die sie vor deren Erschaffung selbst verrichten mussten, nun wieder zu übernehmen hätten. Deshalb ließ er Atramhasis (im GilgameschEpos heißt er Utnapischtim) ein Boot bauen, in dem die Menschheit und die Tiere gerettet wurden, weil sie für die Götter unersetzlich waren. Um aber ihre Vermehrung einzudämmen, gab er ihnen eine begrenzte Lebenszeit, was bei ihrer Erschaffung versäumt worden war. Die Rettung der Menschheit erwies sich als sehr nützlich, denn als Atramhasis nach der Sintflut sein erstes Brandopfer brachte, stürzten sich die Götter darauf wie die Fliegen. Im Gilgamesch-Epos heißt es „,die Götter rochen den süßen Duft, die Götter scharten sich wie die Fliegen um den Opferer“. Noah dankte Gott mit Brandopfern und „der Herr roch den lieblichen 
Geruch und sprach in seinem Herzen: Ich will hinfort nicht mehr die Erde verfluchen um der Menschen willen“ (Genesis 8, Vers 21).

So ist die Sintflut-Legende in postglazialer Zeit über Jahrtausende aus dem kumulativen Gedächtnis von Menschen erwachsen, die in einer Zeit des postglazialen Meeresspiegelanstieges in einer geologisch und klimatisch ausgezeichneten Region lebten, welche für den Ursprung dieser Sintflut-Sage und ihre Weitergabe besonders günstig waren. In diesem Sinne ist es auch nicht wichtig, ob die Flutung des Schwarzen Meeres ein allmähliches oder ein katastrophales Ereignis war. Die Erfahrungen der Menschen, die um das Schwarze Meer siedelten, sind die gleichen, die weltweit alle Menschen in küstennahen Flachländern im Verlaufe des postglazialen Meeresspiegelanstieges gemacht haben.

Durch die christlichen Mythologien wurde die Sintflut-Legende zu einem weltweiten Geschehen verabsolutiert. Die Kirchenväter und Scholastiker des Mittelalters konnten nicht zu objektiv begründeten naturhistorischen Vorstellungen gelangen. Die Quellen ihres Wissens waren die Worte der Bibel, die man nach den Gesetzen der formalen Logistik bearbeitete und die als die absolute Wahrheit galten. Entsprechend wurden alle Zweifel an der Sintflut-Legende geahndet. Noch der französische Aufklärer Buffon (1707-1788), der den bereits von Seneca (-4 bis +64) geäußerten Gedanken eines mehrfachen Wechsels von Zeiten höherer und niederer Wässerstände des Weltmeeres aussprach, verstieß gegen das Dogma der einmaligen Flut zur Bestrafung der sündigen Menschheit. Um die Veröffentlichung einer neuen Ausgabe seines Werkes nicht zu gefährden, musste er sich dem Richterspruch des Kollegiums der Theologischen Fakultät der Sorbonne beugen und alles widerrufen, was sein „Buch über die Bildung der Erde enthält, und überhaupt alles, was gegen die Darstellung des Moses verstößt" (Schulz, 1985).

Heute gibt es Versuche, die Sintflut-Legende mit unserem naturwissenschaftlichen Weltbild in Einklang zu bringen, z. B. die Bemühungen der Kreationisten oder deren verwandte Vereinigungen. Diese Gruppen haben alle ein großes Problem mit der Einordnung ihres Sintflutjahres in das tatsächliche Alter der Erde, und sie ignorieren deshalb auch radiometrische Altersbestimmungen und leugnen grundlegende Fakten der erdgeschichtlichen Entwicklung.

Die Befassung mit der Sintflut-Legende führt uns auch zu einer sehr aktuellen Grundfrage geologischer Entwicklung. Was dominiert die erdgeschichtliche Entwicklung? Sind es kurzfristige Katastrophen oder langfristige Veränderungen? Diese Fragen haben bereits James Hutton (1726-1797) und Charles Lyell (17971875) diskutiert und sich für eine kontinuierliche Entwicklung entschieden, einer Ansicht, der sich auch heute die Mehrheit der Geologen anschließt. Dieses Prinzip der Gleichmäßigkeit (engl. uniformitarian concept) wird natürlich durch Ereignisse wie den Chicxulub-Impakt vor $65 \mathrm{Ma}$ erschüttert. Wir können auch nicht ausschließen, dass massenhaftes Aussterben von Arten $\mathrm{zu}$ anderen Zeiten innerhalb der letzten $500 \mathrm{Ma}$ auf vergleichbare Ereignisse zurückzuführen sind, die wir aber bisher nicht nachweisen konnten. 
Die langfristigen Veränderungen scheinen mir jedoch die dominanten und erdgeschichtlich effektiveren Prozesse zu sein. Sie laufen in Zeiträumen von Jahrmillionen bis Jahrtausenden ab. Aus der Sicht des Geologen sind das überschaubare Zeiten, in denen eine Vielzahl von Einzelereignissen, die durchaus auch lokale oder regionale Naturkatastrophen sein können, eine Gesamtentwicklung ergeben. Ganz analog bei der Sintflut-Legende. Hier ist es das kumulative Gedächtnis einer Gemeinschaft von Menschen, die über Jahrtausende die gleichen Erfahrungen gemacht hat, und die sich dann in der Sintflut-Legende niederschlugen.

\section{Literatur}

Aksu, A. E., Hiscott, R. N., Mudi, P. J., Rochon, A., Kaminski, M. A., Abrajano, T., Yasar, D. (2002): Persistent Holocene outflow from the Black Sea to the Eastern Mediterranean contradicts Noah's Flood hypothesis, GSA Today, May 2002, 4-10

Ballard, R. D., Coleman, D. F. \& Rosenberg, G. D. (2001): Further evidence of abrupt Holocene drowning of the Black Sea shelf, Mar. Geol. 170, 253-261

Bard, E., Hamelin, B., Arnold, M., Montaggione, L., Cabioch, G., Faure, G. \& Rougerie, F. (1996): Deglacial sea-level record from Tahiti corals and the timing of global meltwater discharge, Nature, Vol. 382, 18, July 1996, 241-244

Baumann, H. (1972): Im Lande Ur, Ravenburger Taschenbücher, Band 226

Bird, P. (1978): Finite element modelling of lithosphere deformation: The Zagros collision orogeny, Tectonophysics 50, 307-336

Deutsch, A., Koeberl, C., Blum, J. D., French, B. M., Glass, B. P., Grieve, R., Horn, P., Jessberger, E. K., Kurat, G., Reimold, W. U., Smit, J., Stoffler, D. \& Taylor, S. R. (1994): The impact-flood connection: Does it exist? Terra Nova, 6, 644-650

Hrouda, B. (2003): Vorgeschichte. In: Hrouda, B: Der Alte Orient, Bassermann Verlag, München, 35-55

Kristan-Tollmann, E. \& Tollmann, A. (1992): Der Sintflut-Impakt, Mitt. österr. geol. Ges. 84, 1-63

Langenhorst, F. (2004): Impaktkrater auf der Erde - Spuren des kosmischen Bombardements, GMIT Nr. 15, März 2004, 8-17

Madayska, T., Soffer, O. \& Kurenkova, E. I. (1992): Human occupation. In: Frenzel, B., Pésci, M. \& Valichko, A. A. (eds.): Atlas of Paleoclimates and Paleoenvironments of the Northern Hemisphere, Geographical Research Institute, Hungarian Academy of Sciences, Budapest, plate 63 
Montadert, L., Roberts, D. G.; De Charpal, O. \& Guennoc, P. (1979) : Rifting and subsidence of the northern continental margin of the Bay of Biscay, Initial Reports Deep Sea Drilling Project 48, Scripps Institution of Oceanography, San Diego, 1205-1260

Press, F. \& Siever, R. (2003): Allgemeine Geologie - Einführung in das System Erde, Spektrum Akad. Verlag, Heidelberg, 723 S.

Riem, J. (1925): Die Sintflut in Sage und Wissenschaft, Agentur des Rauhen Hauses, Hamburg, $194 \mathrm{~S}$.

Ryan, W. B. F., Pitman, III, W. C. (1999): Noah's Flood: The new scientific discoveries about the event that changed history, Simon \& Schuster, New York, 319 p.

Ryan, W. B. F., Pitman, III, W. C., Major, C. D., Shimkus, K., Moskalenko, V., Jones, G. A., Dimitrov, P., Görür, N., Sakinc, M., \& Yüce, H. (1997): An abrupt drowning of the Black Sea shelf, Mar. Geol. 138, 119-126

Sarntheim, M. (1972): Sediments and history of the postglacial transgression in the Persian Gulf and northwest Gulf Oman. Mar. Geol., 12, 245-266

Schulz, H. (1985): Leben wir in einem Eiszeitalter? VEB Deutscher Verlag der Wissenschaften, Berlin, 85 S.

Sperling, M., Schmiedl, G., Hemleben, K. C., Emeis, K. C., Erlenkeuser, H. \& Grootes, P. M. (2003): Black Sea impact on the formation of eastern Mediterranean sapropel S1? Evidence from the Marmara Sea.

Palaeogeography, Palaeoclimatology, Palaeooecology 190, 9-21

Stöcklin, J. (1974): Possible ancient continental margins of Iran. In: Burk, C. and Drake, C. L. (eds.): Geology of Continental Margins, Springer, New York

Woolley, C. L. (1930): Ur und die Sintflut, Brockhaus, Leipzig, 137 S. 


\title{
Geologie und Paläontologie im Rahmen der kulturellen Entwicklung
}

\author{
Otto H. Walliser
}

Die Kenntnis von der Vergangenheit und dem Zustand der Erde gereicht dem Menschengeist zur Zierde und Nahrung.

Leonardo da Vinci

\section{Übersicht}

Eine langwierige Geburt: Von der Vorzeit bis ins 18. Jahrhundert. Gedanken über den Beginn - Frühe Empirik versus Mythus - Römisches Intermezzo - Nichts als Naturspiele - Die alles überdeckende Sintflut - Naturalisten

Der Aufbruch im 18. und 19. Jahrhundert. Kommunikationswege - Kartierung: Dokumentation geologischer Gegebenheiten - Stratigraphie: Zeugnis der Erdgeschichte - Evolution: Voraussetzung für Fortschritt - Die Erde: Werden und Gestaltung

Korrelation zwischen geowissenschaftlicher und kultureller Entwicklung. Zeit der Finsternis - Dämmerung, Aufbruch und Durchbruch - Geowissenschaften heute: gewichtiger Faktor der kulturellen Entwicklung 


\section{Prolog}

Der Begriff kulturelle Entwicklung ist hier im weiteren Sinne gebraucht. Er umfasst sowohl die Geisteswissenschaften inklusive Philosophie, Religion, Kunst, Rechts- und Sozialwissenschaften als auch die Naturwissenschaften, Medizin und Technologie als wichtige Parameter der Zivilisation. Alle Teile sind nicht nur durch Übergänge verbunden, sondern eng und unlösbar miteinander vernetzt.

Die kulturelle Entwicklung verläuft phasenhaft, wobei längere Zeitabschnitte der Stagnation oder langsamen Entwicklung von kürzeren Phasen unterbrochen werden, die durch neue, innovative Erkenntnisse und Errungenschaften gekennzeichnet sind. Als Beispiel dafür sei auf die Entwicklung des wohl wichtigsten Faktors der kulturellen Entwicklung hingewiesen, nämlich der Fähigkeit, Wissen $\mathrm{zu}$ vermitteln und zu speichern. Die ursprünglichste Form davon ist die der mündlichen Überlieferung, die mit der Herausbildung der Sprache zwar zunahm, aber in ihrem Umfang natürlicherweise begrenzt ist. Eine innovative Erweiterung der Möglichkeit, Wissen zu speichern, erfolgte dann vor etwa 35000 Jahren durch die zunehmende Fähigkeit bildlicher Darstellungen, wie wir sie von den prächtigen Höhlenmalereien und von Skulpturen aus dieser Zeit und den folgenden 25000 Jahren her kennen. Ein grundsätzlicher Durchbruch wurde aber erst vor zirka 5000 Jahren durch die Herausbildung von Schriften geschaffen. Weitere Meilensteine waren der Buchdruck, insbesondere der mit beweglichen Lettern (Gutenberg, 1455), die Rotationsdruckmaschine (1812) und schließlich, nach der innovativen Einführung von Fotographie und Tonaufzeichnung, die digitale elektronische Speicherung, die seit ihrer Einführung vor zirka 50 Jahren eine rasante Entwicklung erfuhr und zu fast unbegrenzten Möglichkeiten der Wissensspeicherung und -verarbeitung führte. Wie leicht zu ersehen ist, findet mit zunehmender Fähigkeit zur Wissensspeicherung eine deutliche Beschleunigung ihrer eigenen und der damit verknüpften kulturellen Entwicklung statt.

Im vorliegenden Artikel beabsichtige ich nicht, die Geschichte der Geowissenschaften dadurch vorzustellen, dass alle bedeutenden Vertreter aufgeführt werden und deren Beitrag gewürdigt wird. Dies kann in vielen veröffentlichten Darstellungen nachgelesen werden, von denen ich die wohl nur noch in Bibliotheken einsehbaren Monographien von Karl Alfred v. Zittel 1899 und Helmut Hölder 1960, 1986 besonders empfehlen möchte. Die Lebensdaten der im Folgenden angeführten Persönlichkeiten entstammen im Wesentlichen diesen hervorragenden Werken.

Die hier zu behandelnde Frage ist, wie weit die Entwicklung der Geowissenschaften, insbesondere der Geologie und der Paläontologie, in direkter Beziehung oder gar Wechselwirkung zur kulturellen Entwicklung steht. Also zum Beispiel die Frage, ob die Erkenntnisse beziehungsweise Annahmen über die Natur der Fossilien oder über den Aufbau der Erde die Philosophie oder gar die Religionen beeinflussten oder ob es vielleicht umgekehrt war, sei es im positiven oder im negativen Sinne. Die Diskussion darüber ist Gegenstand der letzten beiden Kapi- 
tel. Zuvor wird versucht, die Entwicklung der Geowissenschaften zu skizzieren, ohne jeweils die Verknüpfung mit der kulturellen Entwicklung herauszuarbeiten.

Wenn im Folgenden der Schwerpunkt auf England, Frankreich und Deutschland gelegt und dabei die eigene Universität, also die Georgia Augusta zu Göttingen, besonders berücksichtigt wird, bedeutet dies keinesfalls eine Zurücksetzung anderer Forscher und Forschungsstätten. Die Entwicklung der Geowissenschaften seit der Mitte des 18. Jahrhunderts erfolgte mehr oder weniger gleichzeitig an vielen Orten Europas und Nordamerikas. Sie wäre ohne den intensiven Austausch der Erkenntnisse nicht möglich gewesen.

\section{Eine langwierige Geburt: Von der Vorzeit bis ins 18. Jahrhundert}

\section{Gedanken über den Beginn}

Zählt man zum Metier eines Geowissenschaftlers die Prospektion, also das Aufspüren natürlicher Rohstoffe, so hat dieses sicher schon in der Vorzeit begonnen. Man kann annehmen, dass sich eine eingehende Kenntnis über Vorkommen bestimmter Materialien aus der Erfahrung und dem kollektiven Wissen der steinzeitlichen Bevölkerung heraus entwickelte. Durch zunehmende Bedürfnisse und durch Erweiterung der Anwendungsgebiete dürften sich dann allmählich auf verschiedenen Gebieten besonders Kundige herausgebildet haben.

Frühe Kundige wirkten vielleicht schon in der Steinzeit beim Aufspüren besonderer Gesteinsvorkommen, die zur Herstellung spezieller Artefakte geeignet waren. Ähnliches gilt wohl auch für die Prospektion besonders geeigneter Vorkommen für die Fertigung von Lehmziegeln, wie wir sie seit dem Beginn des Neolithikums bis heute kennen, oder ebenfalls für die schon früh auftretenden Töpferwaren. Ein weiteres Beispiel ist die Beschaffung der nötigen Materialien zur Farbenherstellung für die Ausmalung der ägyptischen Grabkammern, wie neuerliche Funde in der Wüste westlich der Oase Dachla für die Zeit des Pharaos Cheops vor rund 4600 Jahren vermuten lassen. Vielleicht gilt Ähnliches sogar für die viele Jahrtausende älteren Höhlenmalereien zu Beginn und während des JungPaläolithikums.

Mit Sicherheit waren kundige Prospektoren mit speziellen Kenntnissen vom Anfang der Metallzeit an in zunehmendem Maße erforderlich. Sie sind zu jenem Zweig der Geowissenschaften zu zählen, der die Lagerstättenkunde, den Bergbau und Teile der Mineralogie umfasst. Er wird im Folgenden vereinfacht - wenn auch nicht ganz korrekt - unter dem Begriff Montangeologie zusammengefasst.

Zweifellos brachte die Einführung von Metallen revolutionäre Fortschritte, verbunden mit einer allgemeinen Beschleunigung der kulturellen Entwicklung. Man denke nur daran, welch enormer Schub von der Einführung der Bronze erzeugt wurde: Das Zusammenführen der Grundmaterialien Kupfer und Zinn aus meist verschiedenen Gegenden entwickelte und förderte den Handel; neben den 
Bergleuten entstanden neue Berufe wie Gießer, Schmiede, Waffen- und Schmuckhersteller, Transporteure. Der Handel mit weiteren Rohstoffen und Waren wie Salz, Pelzen, Keramik, Bernstein und anderen Schmucksteinen wurde ebenso intensiviert wie die Entwicklung von Schriften. Durch die Vernetzung mit der inzwischen ausgedehnten Landwirtschaft entwickelten sich immer größere, durch Arbeitsteilung gekennzeichnete Gemeinwesen, allerdings auch die bis heute blühenden Rivalitäten zwischen denselben.

Die Einführung und Anwendung von Metall führte also, gemeinsam mit der Herausbildung der Schrift, zum 4. großen Umbruch in der kulturellen Entwicklung. Vorausgegangen waren drei wichtige Innovationen, nämlich die Herstellung von Gesteinsgeräten vor zirka 2 Millionen Jahren, dann die Ausprägung künstlerischer Gestaltung seit etwa 35 000, und schließlich das Aufkommen und die Ausbreitung von Landwirtschaft in der seit dem Ende der Eiszeit vor 10000 Jahren beginnenden so genannten Neolithischen Revolution.

Von der Vorzeit bis heute ist der montanistische Anteil der Geologie unzweifelhaft ein wesentlicher, ja mitbestimmender Faktor der kulturellen Entwicklung. Wir können ihn aber vorerst trotzdem weitgehend aus unserer Betrachtung herausnehmen, da er primär ausschließlich praktisch orientiert war und bis ins 18. Jahrhundert wenig zur Lösung dessen beitrug, was wir als Grundfragen der Geologie und Paläontologie ansehen: was ist die Natur der Erde und der Fossilien; wie und warum haben sie sich entwickelt; wie sind sie miteinander korreliert?

\section{Frühe Empirik versus Mythus}

Als ältestes Zeugnis einer Art entwicklungsgeschichtlichen Denkens kann man die biblische Schöpfungsgeschichte heranziehen, die wohl vor etwa 4500 Jahren entstanden sein dürfte. Eliminiert man einige Unstimmigkeiten und kryptische Stellen, insbesondere den 2. und 4. Tag, so erkennt man eine logisch begründbare Abfolge der Genesis: Nach der Schöpfung von Himmel und Erde am 1. Tag werden am 3. Tag Land und Meer geschaffen als Voraussetzung für die Entstehung von Wasserbewohnern am 5. Tag, denen am 6. Tag die Landbewohner folgen.

Konkrete Anschauungen beziehungsweise Vorstellungen über Fossilien, Veränderungen an der Erdoberfläche, den Aufbau der Erde und deren Stellung im Kosmos erfahren wir erst aus der griechischen Antike. Bereits vor 2500 Jahren zog der Philosoph Xenophanes von Kolophon aus Naturbetrachtungen richtige Folgerungen. Für die Gesteine nahm er eine Entstehung aus schlammigen Sinkstoffen an und deutete die darin enthaltenen Tierreste als Zeugnisse periodischer Überflutungen durch das Meer. Die gleichen Schlüsse zog Herodot aus Halikarnass, der altgriechische Geschichtsschreiber, aus Versteinerungen, die er auf Bergen nahe der Ammons-Oase fand. Erstmals wird durch ihn auch über historische Aspekte, nämlich Veränderungen an der Erdoberfläche, berichtet: „Der größte Teil des genannten Gebietes“ (gemeint ist der untere Nil-Abschnitt) „ist, wie die Priester mir sagen und wie ich annehme, angeschwemmtes Land. Ich 
hatte den Eindruck, dass die zwischen den erwähnten Gebirgen südwärts von Memphis gelegene Ebene einst ein Meerbusen gewesen ist. Warum sollte in der ganzen vor meiner Geburt verflossenen Zeit nicht ein freilich wesentlich größerer Meerbusen durch einen so gewaltigen und so geschäftigen Strom in Land verwandelt worden sein?“ „Würde ein Strom wie der Nil sich in den arabischen Meerbusen ergießen, sollte er nicht in zwanzigtausend Jahren in ein angeschwemmtes Tal verwandelt sein? Ich glaube, dazu wären nur zehntausend Jahre erforderlich.“

Ein Zeitgenosse Herodots, Empedokles von Agrigent, machte sich auch über den Aufbau der Erde Gedanken. Aus dem Vorhandensein heißer Quellen und Vulkane (bei deren Beobachtung er zuletzt in den Ätna gestürzt sein soll) schloss er auf einen feuerflüssigen Erdkern und nicht auf ein Zentralfeuer, wie es früher die Pythagoräer vorgeschlagen hatten. Zur Stellung der Erde in Bezug zu den anderen Himmelskörpern hatte schon Heraklit von Ephesos aufgrund allerdings höchst eigenartiger Spekulationen ein heliozentrisches Weltsystem angenommen und auch erkannt, dass sich die Erde um ihre eigene Achse dreht.

Man sollte annehmen, dass die aufgezählten Erkenntnisse aus dem 6. und 5. vorchristlichen Jahrhundert eine ausgezeichnete Basis für weitere Einsichten bildeten und damit für einen raschen Fortschritt in Richtung echter Wissenschaften. Dem war nicht so. Die naturkundlich denkenden Empiriker und ihre Lehren wurden vielmehr verdrängt oder gar verfolgt von den Vertretern einer spekulativen Philosophie mit abstrusen, ohne Bezug zur Wirklichkeit konstruierten Vorstellungen. Und das für weit über zweitausend Jahre!

Am Anfang standen Erklärungsversuche für die Entstehung der Lebewesen überhaupt, wie dies zum Beispiel der Naturphilosoph Anaximander von Milet durchführte, der nur zirka 50 Jahre vor dem oben erwähnten Xenophanes wirkte. Nach Anaximander entstanden alle Wesen in der aus einem Urstoff („Apeiron“) abgeschiedenen Erde bei der Verdunstung des durch die Sonne erhitzten Wassers. Der etwas jüngere Anaxagoras ließ die Organismen durch Keime aus der Luft in der schlammigen Erde hervorgehen, also ähnlich wie noch 2250 Jahre später Carl Nikolaus Lang.

Aristoteles, der herausragendste Gelehrte der griechischen Antike, Schüler Platos und Lehrer Alexander des Großen, fasste das für ihn gültige Wissen seiner Zeit zusammen, und zwar sowohl jenes auf dem Gebiet der spekulativen Philosophie als auch das auf dem der empirischen Naturbeobachtung. Zu Letzterem war sein eigener Beitrag so groß, dass man ihn zu Recht den Begründer der Zoologie nennen kann. Auch hinsichtlich geologischer Aspekte anerkannte er durchaus manche der früheren Erkenntnisse, wie die gelegentlichen Überflutungen durch das Meer oder die Wirkung der Vulkane. Umso erstaunlicher ist es, dass auch er hinsichtlich der Entstehung der Lebewesen im Prinzip die absurden Spekulationen Anaximanders übernahm. Bei Aristoteles entstanden die niederen Tiere durch eine Urzeugung (generatio spontanae oder generatio aequivoca) im Flussschlamm, in feuchter Erde, aus faulenden Substanzen oder ähnlichem Material. Durch geschlechtliche Fortpflanzung entwickeln sich daraus die höheren Tiere. Dies wie- 
derholt sich in einem geschlossenen Kreis des Werdens und Vergehens, der seit Ewigkeiten besteht und dem alles, also auch die Erde, unterliegt. Diese befindet sich im Mittelpunkt des Weltgebäudes, um den sich die Sterne drehen.

Dem geozentrischen Weltbild des Aristoteles setzte wenige Dezennien später Aristarchos von Samos, der Schöpfer der Trigonometrie, ein wohl begründetes heliozentrisches gegenüber. Etwa gleichzeitig bestimmte Eratosthenes von Kyrene, den Ptolemäus III Euergetes zum Leiter der berühmten Bibliothek in Alexandria berief, den Erdumfang sowie, ziemlich genau, die Ekliptik.

Die Annahme einer Urzeugung bestand mit vielerlei Variationen über zweitausend Jahre lang bis in das 18., teilweise sogar 19. Jahrhundert. Allerdings bestand ein wesentlicher Unterschied zwischen den Lehren der Protagonisten aus der Antike und den Vertretern aus der Zeit der christlichen Kirche. Bei den altgriechischen Philosophen war die „Urzeugung“ eine Erklärung für den allgemeinen Schöpfungsvorgang. Es liegt jedoch nahe zu vermuten, dass ihre Hypothese über die Urzeugung aus dem Schlamm durch die Kenntnis von in der Erde verborgenen Fossilien angeregt, wenn nicht gar bestimmt wurde. Theophrast, Schüler des Aristoteles, nannte in Kenntnis fossiler Knochen die in der Erde befindliche gestalterische Kraft vis plastica. Interessant ist auch, dass in der Urzeugungs-Hypothese eine von primitiven Urformen ausgehende Entwicklung zu höheren Lebewesen angenommen wird. Die Arten sind bei den Vertretern dieser alten griechischen Schule also veränderlich und nicht konstant, wie später aus der mosaischen Genesis fälschlicherweise abgeleitet wurde.

\section{Römisches Intermezzo}

Der Dichter Ovid vermittelt gewissermaßen zwischen der griechischen Antike und der römischen Kaiserzeit. In seinen „Metamorphosen“ legt er dem 500 Jahre zuvor lebenden Pythagoras Folgendes in den Mund: „Das Festland wurde umgewandelt in Meer und das Meer in Festland. Muscheln und Schiffsanker finden sich auf dem Gipfel von Bergen. Täler wurden ausgefurcht von fließendem Wasser, Berge abgewaschen und dem Ozean zugeführt. Spalten wurden durch Erdbeben geschlossen. Erdbeben versenkten Teile des Festlandes und ganze Städte im Meer. Feuer speiende Berge waren einstens untätig, aktive Vulkane werden einstens erlöschen“ (zitiert nach Zittel, S. 5).

Diese sehr fortschrittlichen geologischen Erkenntnisse waren offensichtlich zumindest zu Ovids Zeit schon allgemein anerkannt. Nach dem weit gereisten Geographen Strabo konnten ganze Kontinente aus dem Meere gehoben und wieder in dieses versenkt werden. Dem Land nahe Inseln erklärte er als durch Erdbeben abgetrennte Teile des Festlandes und weiter entfernte Inseln als durch unterirdische Feuer - also als Vulkane - entstanden. Seneca, der Leibarzt des Kaisers Nero, vermutete in seinen „Questiones naturales“, dass Erdbeben durch gewaltige Ausdehnungen von Gasen oder durch den Einsturz von Hohlräumen im Erdinneren entstanden und dass Vulkane durch Kanäle mit unterirdischen Glutherden verbunden seien. 


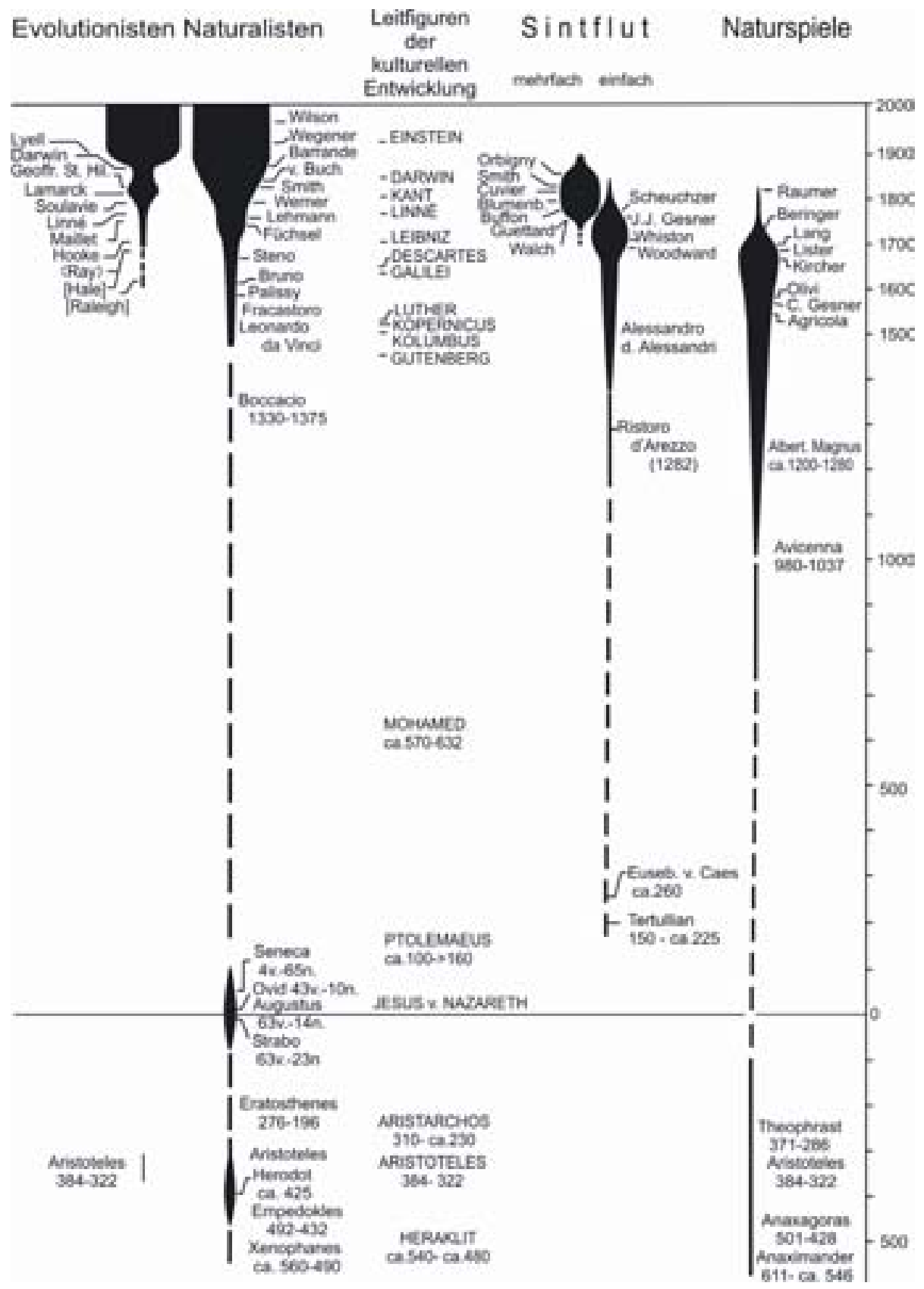

Schema zur Verdeutlichung des relativen Anteils der jeweiligen Lehrmeinungen, mit Nennung einiger wichtiger Vertreter. Deren Lebensdaten sind ab dem 16. Jabrbundert nur im Text aufgefübrt. 
Auf die Natur der Fossilien wird kaum eingegangen. Es wird allerdings berichtet, dass Kaiser Augustus seine Villa in Capri mit allerhand Naturgegenständen ausrüstete. In diesem antiken Naturalien-Kabinett waren auch gewaltige fossile Knochen, wahrscheinlich von Elefanten stammend, die als Überreste von Riesen gedeutet wurden.

Für die Geschichte der Geologie und Paläontologie sind noch zwei weitere Gelehrte aus der römischen Kaiserzeit von Bedeutung: Ptolemaeus und Tertullian. Ptolemaeus führte wieder das geozentrische, „ptolemaeische“ Weltsystem ein. Tertullian, einer der ältesten lateinischen Kirchenschriftsteller, sah, ebenso wie Eusebius von Caesarea, in den Fossilien auf dem Festland Zeugnisse für die große biblische Flut.

\section{Nichts als Naturspiele}

Im Gegensatz zu den antiken Philosophen bezogen der islamische Philosoph Avicenna (= Ibn Sina) und viele christliche Autoren ihre abstrusen Hypothesen ausschließlich auf die Natur der Fossilien. Diese waren bei Johann Baptista Olivi in seiner 1584 erschienenen Beschreibung von Fossilien aus einem Museum in Verona nur Naturspiele, lusus naturae. Conrad Gesner (1516-1565) bezeichnete sie als Figurensteine (lapides figurati). Avicenna ließ sie, in Anlehnung an die aristotelische Schule, durch eine geheimnisvolle vis plastica hervorbringen. Von einer virtus formativa sprach Albertus Magnus (= Albert von Bollstaedt), der 1931 heilig gesprochene große Gelehrte des 13. Jahrhunderts, in Anlehnung an Avicenna. Athanasius Kircher (1602-1680) ließ einen spiritus plasticus oder architectonicus wirken. Carl Nikolaus Lang (1670-1741), ein damals berühmter Arzt und Ratsherr in Luzern, veröffentlichte 1708 eine „Historia lapidum figuratorum Helvetiae“, in der er auf 163 Tafeln Kristalle, Stalaktiten und die verschiedenartigsten Fossilien darstellte. Er verglich Letztere mit rezenten Bewohnern der Meeresküste und kommt zu dem überraschenden Ergebnis, dass die die Gesteine befruchtende aura seminalis von der Hochsee und nicht von der Küste stammen müsste, da er viele Fossilien, wie z. B. Ammonshörner, nicht an den Gestaden fand. C. von Raumer hielt die Fossilien selbst noch 1817 für nie belebt gewesene, vorzeitig abgebrochene Versuche der Natur.

Auch Agricola (= Georg Bauer, 1494-1555) sah die Schalen tragenden Fossilien als aus verhärtetem Wassergemenge, einer materia pinguis, hervorgegangen an. Dies ist umso erstaunlicher, als er bei Pflanzen, Knochen und Fischen durchaus einen organischen Ursprung vermutete. Ihre Umwandlung in Gestein bewirkte ein succus lapidesceus. Agricola, dieser gelehrte Arzt, wurde von ,seinen Zeitgenossen als Zierde Deutschlands bezeichnet“" und A. G. Werner nannte ihn den „Vater aller Bergwerksgelehrten und Schöpfer aller mineralogischen Kritik“, der ,an Schärfe der Beobachtung und Präzision der Darstellung unter den Mineralogen aller Zeiten eine hervorragende Stelle“ einnimmt (zitiert nach Zittel, S. 17). Agricola schuf übrigens den Begriff Fossil, worunter er - und viele andere nach ihm noch nahezu 200 Jahre lang - alles umfasste, das aus der Grube (lat. 
fossa) zu bergen ist: Mineralien ebenso wie Versteinerungen von ehemaligen Lebewesen. Letztere wurden von späteren Autoren meist als Petrefakten oder, wie heute, als Fossilien (sensu stricto) bezeichnet.

\section{Die alles überdeckende Sintflut}

Wie schon in der Antike gab es auch nach der Zeitenwende neben den mythischen Vorstellungen solche, in denen Fossilien als Überreste ehemals lebender Organismen erkannt wurden. Da es sich in den meisten Fällen offensichtlich um ehemalige Meerestiere handelte, stand im Vordergrund der Diskussion die Frage, wie diese auf das Festland gelangen konnten. In einer Zeit, in der religiöse Aspekte grundlegende Bedeutung hatten, lag es nahe, die Sintflut aus der Bibel zur Erklärung heranzuziehen. Diese weltweit wirkende mosaische oder noachische Überschwemmung, die davor schon in der deukalischen Flut ihre Entsprechung hatte, spielte auch in manch anderen Religionen und Mythen eine Rolle. Da man seinerzeit noch nicht wie heute eine geologische Erklärung hatte, nämlich den rapiden Anstieg des Meeresspiegels nach der letzten Eiszeit, sah man in der Flut eine Strafaktion der göttlichen Kräfte, deren Wiederholung wegen Missachtung der göttlichen Gebote jederzeit drohte.

Aus der großen Zahl der Diluvianer, also derjenigen Autoren, welche der biblischen Überschwemmung anhingen, sollen nur einige wenige genannt werden. So der bald nach Tertullian lebende Eusebius von Caesarea, der vom Vorkommen von Fossilien auf den Gipfeln des Libanon auf eine ungeheuer hohe Sintflut schloss. Das Zusammenschwemmen von Fossilien auf dem Festland schrieb 1000 Jahre später auch Ristoro d'Arezzo der Sintflut zu. Alessandro del Allesandri legte, um Fossilfunde auf den kantabrischen Bergen zu erklären, der Sintflut eine Veränderung der Rotationsachse der Erde als Ursache zugrunde.

Die genannten und viele weitere Autoren begnügten sich damit, die Sintflut durch das Vorkommen von Fossilien auf dem Festland zu beweisen oder, umgekehrt, dieses Vorkommen der Fossilien durch die Sintflut zu erklären. Über die Ursache der Sintflut spekulierte dagegen der englische Professor John Woodward (1665-1722) in seiner 1695 erschienenen „Natural History of the Earth“. Danach war das Erdinnere eine riesige Wasserkugel. Als die darüber liegende Rinde zerbrach, trat das Wasser aus und verursachte die Sintflut. Dabei wurde die zuvor bestehende Kohäsion ausgesetzt, sodass sich die Gesteine - mit Ausnahme der Muscheln und sonstigen Meerestiere sowie der Metalle - auflösten. Bei der Erneuerung der Kohäsion bildete sich eine neue Erdkruste, in welche die Fossilien und Metalle eingeschlossen wurden: zuunterst die schweren, darüber, in der Kreide, leichtere Schalentiere und Seeigel, darüber Pflanzen sowie die Schalen der leichteren Land- und Süßwassertiere. Mit dieser phantastischen Geschichte konnte Woodward einige beobachtbare Phänomene erklären, ohne in Widerspruch zur Bibel zu geraten: die Fossilien sind im Gestein und nicht nur auf dessen Oberfläche; man kann anhand der Versteinerungen verschiedene, übereinander liegende Schichten unterscheiden; es gab also auch Lebewesen, die aus der 
Gegenwart nicht bekannt sind. Nach Woodward sind diese nicht etwa ausgestorben, sondern in die Tiefsee abgewandert. Im Übrigen schloss er aus der Zartheit der eingeschlossenen Pflanzen und dem Vorkommen an „Fischrogen“ (wohl Oolithe), dass die Sintflut im Monat Mai stattfand. Mit nicht minder skurrilen Hypothesen kam Woodwards Zeitgenosse William Whiston (1666-1695) auf den 18. November des Jahres 2349 vor Christus als Datum der Sintflut. Dass der 1755 als Polizey Kommisarius an die Universität Göttingen berufene Bergrat J. H. G. Justi noch in seiner 1771 erschienenen „Geschichte des Weltkörpers“ darüber spottete, ist nicht nur aus heutiger Sicht kaum verwunderlich.

Rund 50 Jahre zuvor hatte Johann Jacob Scheuchzer (1672-1733), Arzt und Mathematik-Professor am Gymnasium in Zürich, Woodwards Werk ins Lateinische übersetzt und konvertierte dadurch vom Verfechter der Naturspiele-Hypothese zum begeisterten Diluvianer. Davon zeugt das von ihm als Homo diluvii testis beschriebene Fossil. Dass es sich jedoch nicht um das „Bein-Gerüst eines in der Sündflut ertrunkenen Menschen“ handelte, erkannte wenig später der Züricher Naturgelehrte und Mediziner H. J. J. Gesner. Er interpretierte es als Überrest eines Welses. Erst Cuvier, von dem später noch mehrmals die Rede sein wird, erkannte es als Riesensalamander, der heute den Namen Andrias scheuchzeri führt.

Woodwards Erkenntnis, dass die Verteilung der Fossilien auf eine gewisse Abfolge, modern ausgedrückt auf eine biostratigraphische Gliederung, schließen lässt, verdichtete sich im 18. Jahrhundert in rasch zunehmendem Ausmaß. Dabei wurde mehr und mehr klar, dass ältere Faunen immer wieder durch jüngere ersetzt wurden. Um dies irgendwie in Einklang mit den kirchlichen Dogmen zu bringen, erklärte man es dahingehend, dass eben nicht nur eine einzige Sintflut geschehen war, sondern mehrere, zuletzt, bei Cuvier, sogar viele. Bemerkenswert ist, dass die meisten von Cuviers Katastrophen heute wieder als Zeiten globaler, häufiger oder massenhafter Extinktionen bestätigt und in den meisten Fällen auf Schwankungen ozeanischer Parameter, zum Beispiel des Meeresspiegels, zurückgeführt werden können.

\section{Naturalisten}

Der Begriff Naturalismus wird in den verschiedenen Disziplinen, von der Philosophie bis zur Naturwissenschaft, zum Teil stark voneinander abweichend definiert beziehungsweise angewendet. In unserem Zusammenhang werden als Naturalisten diejenigen Autoren bezeichnet, die empirische Naturbeobachtung und - Forschung betreiben und die Ergebnisse entweder ohne Interpretation darstellen oder, falls Erklärungsversuche oder Hypothesen aufgestellt oder verwendet werden, diese nicht opportunistisch an bestehende Dogmen anpassen. Sie unterscheiden sich in dieser Hinsicht von vielen derjenigen Diluvianer, die ebenfalls Naturstudien betrieben.

Abgesehen von den schon genannten antiken Autoren erkannte auch Giovanni Boccaccio, der Dichter des Decamerone, im 14. Jahrhundert, dass die fossilen Meeresmuscheln in der Toscana von Tieren herrühren, die einst dort 
gelebt hatten. Einen Höhepunkt der naturalistischen Erkenntnisse erreicht rund 150 Jahre später das Universalgenie Leonardo da Vinci. Er wendet sich eingehend gegen die Mystiker, die behaupten, „dass die Muscheln in den Bergen von der Natur mit Hilfe der Gestirne erzeugt worden sind“, und fragt sie, auf welche Weise „diese Gestirne Muscheln von verschiedener Größe, verschiedenem Alter und verschiedener Art an demselben Ort geschaffen haben" (zitiert nach Lücke, S. 218/219). Gegen die Sintflut-Hypothese bringt er zahlreiche Argumente vor. So zum Beispiel, dass die Sintflut ,in 40 Tagen und Nächten durch einen unaufhörlichen, über die ganze Erde verbreiteten Regen verursacht wurde“. Die Muscheln auf dem heutigen Festland gleichen aber marinen Formen und sind nicht nur auf der Oberfläche, sondern überwiegend in den Gesteinsschichten eingeschlossen. Dort finden wir sie ,alle noch zusammen und sehen auch die Schnecken, die Tintenfische und die anderen Muscheln noch im Tode so zusammenliegen, wie sie in Scharen miteinander lebten und ... wie wir es jeden Tag an den Meeresgestaden beobachten könnten“. Mit dieser ihm eigenen aktualistischen Betrachtungs- und Erklärungsweise kam Leonardo auch bei anderen geologischen Phänomenen, wie zum Beispiel dem Vorgang der Fossilisation oder dem Materialtransport in Flüssen, zu plausiblen Erklärungen. Leider veröffentlichte er seine Überlegungen und Ergebnisse nicht. Zudem hatte er sie in Spiegelschrift geschrieben. Damit waren sie für die Entwicklung der Geowissenschaften verloren, zeugen aber von der Genialität dieses Renaissance-Künstlers.

Ganz ähnliche Überlegungen wie Leonardo äußerte dessen jüngerer Zeitgenosse Hieronymus Fracastoro (1483-1553). Durch Muscheln, die beim Bau der Zitadelle von San Felice in Verona gefunden wurden, kam er zu der Erkenntnis, dass sie ehemals am Einbettungsort gelebt hatten und somit sowohl die vis plastica als auch die Sintflut zur Erklärung nicht in Frage kamen. Auch Bernhard Palissy (1510-1589), Glasmaler und Kunsttöpfer in Paris, wandte sich gegen Mystiker und Diluvianer. Als Amateur, der weder Griechisch noch die damalige Wissenschaftssprache Latein konnte, kam er durch seine Naturbeobachtung zu entsprechenden, fortschrittlichen Deutungen wie Leonardo da Vinci.

Von den an Zahl zunehmenden Naturalisten sei noch der dänische Arzt Nicolaus Steno (= Niels Stensen, 1638-1687) erwähnt. Er war, von der protestantischen zur katholischen Kirche übergetreten, im Dienste der Medici in Florenz. Als Generalvikar für Niedersachsen starb er in Schwerin, wurde aber auf Anordnung von Großherzog Cosimo III. nach Florenz überführt und in der Kathedrale St. Lorenzo beigesetzt. Vielleicht hingen seine für die damalige Zeit unerhörten medizinischen Erkenntnisse - zum Beispiel, dass das Herz nichts als ein Muskel ist - ebenso wie die geologisch-paläontologischen, mit seiner Freundschaft zu dem als Atheist geltenden Philosophen Spinoza (1632-1677) zusammen. In seinem 1669 erschienenen Werk „De solido intra solidum naturaliter contento“ erhärtete Steno durch eingehenden Vergleich den schon von Leonardo da Vinci sowie 1517 von Fracastoro und 1616 von Colonna geäußerten Verdacht, dass die sogenannten Glossopetren Haifischzähne sind und ebenso wie fossile Muscheln und Schnecken der Toscana Überbleibsel einer vorzeitlichen Fauna sind, die an 
ihrem Fundort gelebt hatte. Er verglich sie mit rezenten Arten und versuchte, die verschiedenen Erhaltungsformen zu deuten. Darüber hinaus wies er darauf hin, dass die höher liegenden Schichten jünger sind als die unteren sowie dass die einzelnen Schichten zum Teil erhebliche Unterschiede aufweisen.

Die bisher genannten Naturalisten waren ihrer Zeit zum Teil weit voraus, ohne dass sie jedoch einen besonderen Einfluss auf die Entwicklung der Erdwissenschaften hatten. Erst die ab dem 18. Jahrhundert aufzuführenden Naturalisten bewirkten den Auf- und Durchbruch der Geowissenschaften.

\section{Der Aufbruch im 18. und 19. Jahrhundert}

Dieser Abschnitt in der Entwicklung der Geowissenschaften wird häufig die „heroische Zeit" genannt. Ich möchte lieber von der Zeit des Aufbruchs sprechen, und zwar Aufbruch in doppeltem Sinne: zum einen das Aufbrechen alter, tradierter, festgefahrener und den Fortschritt hemmender Strukturen, und damit gleichzeitig Aufbruch hin zu Erkenntnissen und Verfahrensweisen, die zu einer wirklichen Wissenschaft vom Entstehen und Werden der Erde und ihrer Lebenswelt führen.

Als besonders hervorragende Vertreter der Aufbruchszeit werden häufig James Hutton, Abraham Gottlob Werner und Charles Lyell sowie William Smith, Georges Baron de Cuvier und schließlich Charles Darwin genannt. Ohne Zweifel trugen diese „Heroen“ entscheidend dazu bei, die geologisch-paläontologischen Teilgebiete der Naturkunde zur Anerkennung als Naturwissenschaften zu führen, beziehungsweise ihnen zum Durchbruch zu verhelfen.

Die großen Verdienste der eben Genannten beruhen jedoch zum Teil eher auf der umfangreichen Anwendung und Verbreitung neuer Erkenntnisse als auf deren Gewinnung selbst. Ihnen allen waren Wegbereiter vorausgegangen. Damit erhebt sich die Frage, wessen geistige Leistung höher einzuschätzen, beziehungsweise wem das größere Verdienst zuzuschreiben ist. Waren es jene, die ganz am Anfang des Aufbruchs, in der Zeit der Dämmerung, standen und aus ihrer Beschäftigung mit der Natur die wegweisenden Erkenntnisse und Deutungen bezogen, denen aber nicht selbst der Durchbruch gelang? Oder waren es die nachfolgenden Autoren, welche die Erkenntnisse ihrer Vorgänger vertiefen, erweitern und wirksam verbreiten konnten? Vielleicht lässt sich eine Beantwortung aus den folgenden Abschnitten ableiten.

\section{Kommunikations-Wege}

Neben der Möglichkeit der Speicherung des Wissens ist die Art, Weise und Intensität der Möglichkeit zu kommunizieren eine der ganz wichtigen Faktoren der Entwicklung einer Wissenschaft. Wie war dies zu Beginn des Umbruchs, also im späten 18. Jahrhundert? Damals gab es weder entsprechende Fachzeitschriften noch Gesellschaften. Sie wurden - nach und neben einigen jetzt aufkommenden 
naturhistorischen Vereinen - erst im 19. Jahrhundert gegründet: 1807 die Geological Society of London, 1830 die Société Géologique de France, 1848 die Deutsche Geologische Gesellschaft. Ergebnisse konnten gegebenenfalls in den Zeitschriften der bereits bestehenden wissenschaftlichen Akademien veröffentlicht werden oder in denen der nun immer mehr aufkommenden, regional begrenzten naturhistorischen Vereine oder in Form von Büchern, wobei besonders Lehrbücher eine wichtige Rolle spielten. Daneben kam der individuellen Kommunikation durch direkte Kontakte oder durch Briefe eine ganz wichtige Aufgabe zu. Um dies zu verdeutlichen, können die Verhältnisse in Göttingen herangezogen werden.

Obwohl das offizielle Gründungsdatum der Universität Göttingen im Jahr 1737 liegt, wurde die Matrikel bereits 1734 eröffnet. Schon 14 Tage davor hielt Samuel Christian Hollmann, Professor der Philosophie und Physik, die erste Vorlesung. Er war es, der als Erster in Deutschland die ganze Naturgeschichte lehrte, also neben Chemie und Physik, Botanik und Zoologie auch das Wissen über die Erde, die Gesteine, Mineralien und die als Petrefakten bezeichneten Versteinerungen organischen Ursprungs. Mitte des 19. Jahrhunderts schrieb Hollmann über „Ossium fossilium... in praefectura vicina Herzbergensi“. In den hinzugefügten Abbildungen versah er die fossilen Knochen mit Bezeichnungen, wie sie für rezente Wirbeltiere benutzt wurden, und führte damit einen Vergleich der Anatomie durch. Die von Hollmann gefundenen fossilen Knochen, unter anderem vom Wollhaar-Nashorn, gingen in das damalige Naturalienkabinett ein und sind zum Teil in der Sammlung des Geowissenschaftlichen Zentrums noch erhalten (freundl. Mitt. Dr. H. Jahnke).

Nun zu Johann Friedrich Blumenbach (1752-1840). Seine damalige Bedeutung spiegelt sich darin wider, dass er 1825, zur Zeit seines 50-jährigen DoktorJubiläums, Mitglied in weltweit 55 Akademien und wissenschaftlichen Gesellschaften war und als Nestor der deutschen Naturforscher, als „Magister Germaniae" geehrt und bei der Aufnahme in die Kaiserliche LeopoldinoCarolingische Akademie mit dem akademischen Beinamen Aristoteles II. versehen wurde. Eine lange Liste weiterer Ehrungen, verliehener Titel und Orden ist bei Dougherty (1984) aufgeführt.

Blumenbach kam 1772 als Magister der Medizin aus Gotha nach Göttingen. Ein Empfehlungsschreiben des Gothaer Rektors Geisler an Christian Gottlob Heyne (1729-1812), den damals führenden Gelehrten der jungen Universität, brachte ihm die Aufgabe ein, das 1772 erworbene Naturalien- und Münz-Kabinett von Christian Wilhelm Büttner (1716-1801), dem „Stein-Büttner“, zu ordnen. 1775 wurde er aufgrund der Dissertation „De generis humani varietate nativa“ zum Dr. med. promoviert. Selle, 1937, bemerkt dazu, dass man nicht unbedingt sagen kann, dass Blumenbach „eigene Gedanken auf dem Gebiete der Rassenkunde hätte, auch nicht in allen anderen von ihm vertretenen Fächern. Von Büttner wurde Blumenbach zu seiner Dissertation angeregt, die ihn mit einem Schlage zu einem berühmten Manne machte“ und ihm später den Titel „Vater der Anthropologie" einbrachte. 
1776 wurde Blumenbach zum Aufseher der nun als „Königlich Akademisches Museum“ geführten Sammlungen sowie zum Außerordentlichen Professor und 1778 zum Ordentlichen Professor der Medizin und Arzneywissenschaft bestellt. Im selben Jahr heiratete er Louise Amalie Brandes, Schwägerin Heynes, Tochter des hannoverschen Geheimen Kanzleisekretärs Georg Brandes, „des wahren Lenkers der Göttinger Universität“ (Dougherty, 1984). Blumenbach übernahm von Büttner auch dessen Kolleg über Naturgeschichte, das er „in mehr als 100 Semestern zu wahrem europäischem Ruhm bringen sollte“ (Selle, S. 143). Begleitend zur Vorlesung erschien erstmals 1779 sein „Handbuch der Naturgeschichte“, das bis 1830 zwölf Auflagen sowie Übersetzungen in mehrere Sprachen erfuhr.

Blumenbach unternahm selbst nur wenige Reisen. 1783 besuchte er Goethe in Weimar, G. E. Haller, den Mitbegründer der Königlichen Gesellschaft der Wissenschaften zu Göttingen, in Bern und Ch. Bonnet in Genf. 1791/92 weilte er zwei Monate in London am British Museum. Er traf dort den Naturforscher Sir Joseph Banks (1743-1820), der James Cook auf dessen erster Weltumsegelung begleitet hatte, sowie weitere Mitglieder der Royal Society, deren Fellow er selbst ein Jahr später wurde. Sein oberster Dienstherr, König Georg III., empfing ihn zur Audienz, ebenso wie auch Königin Charlotte. Dies geschah offensichtlich auf Empfehlung von de Luc.

Andrée de Luc (1727-1813) wurde in Genf geboren. 1768 beteiligte er sich an den politischen Kämpfen in Genf, von wo er nach Bern und Paris gesandt und dort 1770 in den Großen Rat gewählt wurde. Später fungierte er als Reisebegleiter und Vorleser der Königin Charlotte. 1798 erfolgte seine Ernennung zum Honorar-Professor für Geologie an der Universität Göttingen, ohne dort jemals tätig zu sein. Er war zu Lebzeiten als Polyhistor hochgeachtet und stand mit fast allen namhaften Vertretern von Meteorologie, Physik, Cosmologie, Chemie, Philosophie, Theologie, Sozialpolitik, Geographie und Geologie in brieflichem Kontakt. So zum Beispiel auch mit Georg Christoph Lichtenberg (1742-1799), dem Göttinger Physik-Professor und Meister des Aphorismus. Allerdings, so Zittel 1899, S. 109, lohnt es sich hinsichtlich de Luc ,nicht, auf die Anschauungen dieses außerordentlich emsigen, aber flüchtigen Beobachters und phantastischen Vielschreibers näher einzugehen; de Luc's Publicationen sind zum größten Theile der verdienten Vergessenheit anheimgefallen“.

Ebenso wie de Luc pflegte Blumenbach eine äußerst umfangreiche Korrespondenz, in der er die wissenschaftlichen Probleme und Erkenntnisse seiner Zeit diskutierte. Dabei bat er häufig, besonders bei Forschungsreisenden, um Vervollständigung seiner Sammlung, besonders auch von Schädeln der verschiedenen Gegenden und Rassen. So sandte ihm Georg Thomas von Asch (1729-1807) 1797 den Schädel eines persischen Bey. Von Asch war 1750 in Göttingen bei Albrecht von Haller promoviert worden. Anschließend kehrte er in seine Vaterstadt St. Petersburg zurück, wo er unter der Zarin Elisabeth angestellt und besonders unter Katharina II., der Großen, vom Stadtphysikus zum geadelten Staatsrat und Ehrenmitglied der Kaiserlichen Akademie der Wissenschaften avancierte. 
Alexander von Humboldt (1769-1859) sandte seinem ,,theuren Lehrer und Freund“ Blumenbach 1806 zwar keinen Schädel, aber wenigstens das Bild eines Riesen-Mestizen aus Mexiko. Es gelang Blumenbach auch durch Georg III. für das Academische Museum einen beträchtlichen Teil der ethnographischen Sammlung zu erwerben, die von Johann Reinhald Forster (1729-1798) und seinem Sohn Georg (1754-1794) auf der zweiten Cook'schen Weltumsegelung zwischen 1772 und 1775 hauptsächlich in der Südsee zusammengebracht wurde. Humboldt und Forster standen nicht nur über Blumenbach, sondern auch direkt miteinander in Verbindung. Sie unternahmen 1790 gemeinsam eine Reise durch die Niederlande, Großbritannien und Frankreich. Selbstverständlich waren beide Wissenschaftler ihrerseits jeweils auch mit anderen Naturforschern in direkter Verbindung. Dies zeigt beispielhaft die enge Vernetzung der Kommunikationswege in der Zeit des Aufbruchs. Von den vielen Briefpartnern Blumenbachs möchte ich nur noch Goethe und Cuvier nennen, da er mit diesen in speziellen Beziehungen stand.

Johann Wolfgang von Goethe (1749-1832) wurde von Blumenbach mehrfach in Weimar besucht und traf diesen auch bei seinen zwei längeren Aufenthalten, 1783 und 1801, in Göttingen. Er besuchte dort nicht nur die seinerzeit berühmte Bibliothek und das Museum, sondern sammelte auch mit seinem Sohn August auf dem Hainberg oberhalb Göttingens Fossilien, besonders Ceratiten - die er allerdings nachts zum Teil als Wurfgeschosse gegen bellende Hunde benützte. Goethes Interesse für die Erforschung der Natur wurde durch die ihm anvertraute Leitung des Ilmenauer Bergwerks geweckt. Dementsprechend führte er ,geognostische" Untersuchungen in der Gegend von Karlsbad und Franzensbad sowie im Fichtelgebirge durch und korrespondierte neben anderen auch mit Blumenbach über allgemeine geologische Fragen, worüber er auch mehrere Abhandlungen verfasste. Dabei wandte er sich auch gegen die auf James Hutton (17261796) zurückgehende plutonistische Hypothese, nach der die wesentlichen Gestaltungskräfte vom Innern der Erde ausgehen. Goethe hing dagegen, wie auch Blumenbach, der von Abraham Gottlob Werner (1749-1817) ausgelösten Hypothese der sogenannten Neptunisten an. Danach kristallisierten aus einem heißen Urozean zuerst Granite aus und dann in einer bestimmten Reihenfolge die anderen Gesteine. Er wandte sich allerdings sehr ungehalten auch gegen extreme Vertreter der Neptunisten, da er wie Blumenbach Vulkane als durch unterirdische Kohlenlager gespeiste Erscheinungen an der Oberfläche der Erdkruste deutete.

Georges Baron de Cuvier (1769-1832) wurde in der von 1397 bis 1793/1803 württembergischen Exklave Mömpelgard (Montbéliard) geboren. Er erfuhr seine Ausbildung kurze Zeit am berühmten Tübinger Stift und dann von 1784 bis 1788 an der Karlsschule, der Militärakademie in Stutgart, an der ein Dezennium zuvor auch Friedrich Schiller (1759-1805) studiert hatte. Cuvier widmete sich dem Studienfach Administration, in dem auch Naturgeschichte enthalten war. Seine „ersten Ideen in philosophischer Naturgeschichte“ erhielt er nach eigener Bezeugung allerdings von K. F. Kielmeyer, der erst später zum Lehrer an der Karlsschule avancierte. Cuvier ging nach dem Studium von 1788-1795 als 
Hauslehrer in die Normandie, wo er die französische Revolution von ferne erlebte und 1793 die französische Staatsbürgerschaft erhielt. Er benützte seinen Aufenthalt zum Studium der Flora und Fauna sowohl des Landesinneren als auch der Küste. Dabei kam er, wie er seinem Stuttgarter Freund Pfaff schrieb, zu der Erkenntnis, dass er „nichts anderes als ein Naturalist“ sein wollte. Dieser Wunsch wurde ihm erfüllt, als er 1795 an den Jardin des Plantes in Paris berufen wurde, an dem auch G. de Saint-Hilaire und Lamarck tätig waren. Cuvier wurde bald Direktor des Jardin und reussierte auch im staatlichen Bereich bis hin zum Pair von Frankreich (1831, unter König Louis Philipp). Schon unter Napoleon erreichte er höchste Stellen der Kultusverwaltung. Diesem Umstand ist es sicherlich $\mathrm{zu}$ verdanken, dass sein Freund Bumenbach sowie Professor Martens am 28.09.1807 als Vertreter der Göttinger Universität von Napoleon empfangen wurden. Dabei soll dieser gesagt haben, dass eine Universität, an der ein Blumenbach lehrt, nicht - wie die anderen Hochschulen der besetzten Gebiete - geschlossen werden kann, zumal die Universität Göttingen weder nur Hannover oder nur Deutschland gehöre, sondern der Welt.

Blumenbach und Cuvier verband das Interesse an den fossilen Elefantiden sowie die innovative Untersuchungsmethode der vergleichenden Anatomie. Meistens wird Cuvier als Begründer derselben und damit als „Vater der wissenschaftlichen Paläontologie" genannt, wobei er selbst bezüglich der vergleichenden Anatomie Blumenbach als seinen Lehrmeister bezeichnete. Dass Blumenbach auf Vorläufern aufbauen konnte, wurde oben bei Hollmann angedeutet. Dieser hatte schon 1751 Knochen der beiden von Blumenbach 1799 mit wissenschaftlichem Namen versehenen großen Säugetiere, Wollhaar-Nashorn und das zu den Elefanten gehörende Mammut, abgebildet und vergleichend beschrieben. Cuvier, der mit Blumenbach die Fundstellen Hollmanns aufsuchte, dehnte seine Forschung auf alle damals bekannten fossilen und rezenten Elefanten sowie weitere Tetrapoden einschließlich Saurier aus und verhalf damit der vergleichenden Anatomie in der Paläontologie zum Durchbruch. Dies wurde von beiden, Cuvier und Blumenbach, durch ihre Vorlesungen und Lehrbücher über vergleichende Anatomie zusätzlich gefördert.

Obwohl Lamarck bereits die Evolution durch Veränderung der Arten lehrte, war der am gleichen Institut wirkende Cuvier von deren Konstanz, also ihrer Unveränderlichkeit, überzeugt. Er, ebenso wie Blumenbach, gingen „,von einem kaum übersehbaren Heer von Versteinerungen präadamitischer Geschöpfe der Vorwelt“ aus, „die einst durch eine allgemeine Erdkatastrophe vertilgt worden“ waren (Blumenbach 1799, Nr. 41). Die Lebenswelt nach der Katastrophe ist also eine Neuschöpfung. Cuvier erkannte jedoch, dass nicht nur eine, nämlich die mosaische Sintflut gewirkt hatte. Vielmehr war eine größere Anzahl von Katastrophen notwendig, um die im Verlauf der Erdgeschichte auftretenden Verschiedenheiten der Lebenswelten erklären zu können. Er begründete damit die später so genannte Katastrophen-Theorie. Cuvier und Blumenbach erkannten, beziehungsweise anerkannten damit das, was später Biostratigraphie genannt wurde: die Kennzeichnung und Gliederung der erdgeschichtlichen Schichtenfolge durch 
zeitlich unterschiedliche Faunen und Floren, die jeweils für die aufeinander folgenden Abschnitte charakteristisch sind.

Die Göttinger Verhältnisse stehen ganz allgemein für die damalige Art und Weise der Kommunikation zwischen den Naturforschern. Zwei wichtige Punkte sind dabei hervorzuheben: zum einen dass die Kommunikationswege zwischen den damals in noch relativ geringer Anzahl tätigen Naturforschern in vielfacher Weise eng vernetzt waren. Zum anderen war die Zeitdauer für die Verbreitung und Diskussion neuer Erkenntnisse im Vergleich zu den heutigen Möglichkeiten gar nicht entscheidend länger: Postkutsche und Schiff, also Tage bis Wochen, anstelle von Auto, Eisenbahn und Flugzeug, also Stunden bis Tage; persönliche Besuche und Briefe anstelle von E-mails; Abschriften und relativ wenige Publikationen anstelle von Internet und einer Unzahl von Zeitschriften und Büchern.

Wie sich unter den beschriebenen Verhältnissen der Aufbruch gestaltete, soll besonders am Beispiel der Stratigraphie sowie mit einigen Bemerkungen über weitere Teilgebiete der Geowissenschaften aufgezeigt werden.

\section{Kartierung: Dokumentation geologischer Gegebenheiten}

Eine wichtige Grundlage für die Geowissenschaften ist die Kenntnis der räumlichen und zeitlichen Verteilung erdgeschichtlicher Dokumente, wie zum Beispiel die Verbreitung bestimmter Gesteinsarten, der Lagerungsverhältnisse und tektonischer Strukturen sowie sedimentologische, geochemische und lagerstättliche Daten. Dies kann durch eine kartographische Erfassung erreicht werden.

Die ältesten geologischen Karten stammen aus der Mitte des 18. Jahrhunderts. Auf Anregung von Martin Lister (1638-1711) fertigte Christopher Packe 1743 eine erste, allerdings noch recht unvollkommene Karte des östlichen Kent. Wesentlich beeindruckender ist die 1746 von Jean Etienne Guettard (1715-1786) vorgelegte geologische Karte von Frankreich. Er verglich auch den Nordwesten Frankreichs mit dem südöstlichen England und stellte dabei eine Übereinstimmung zwischen den Fossilien beider Regionen fest.

In der zweiten Hälfte des 18. Jahrhunderts wurden schon häufiger geologische Karten erstellt. Erwähnt sei der erste Versuch durch Georg Christian Füchsel (1722-1773), der sich über die Stratigraphie und die Geologie Thüringens große Verdienste erwarb, ebenso wie Johann Karl Wilhelm Voigt (1752-1821), der eine kolorierte petrographische Landkarte und eine geologische Beschreibung des Thüringer Waldes erstellte. Voigt war ein früher Schüler von Abraham Gottlob Werner (1749-1817), dem Inhaber des ersten Lehrstuhls für Geologie an der sächsischen Bergakademie in Freiberg. Ein späterer Schüler, Johann Friedrich Wilhelm Charpentier (1786-1855), der später die erratischen Blöcke im Alpenvorland erstmals richtig durch Gletscher-Transport erklärte, schuf 1775 eine umfassende geologische Karte der chursächsischen Lande und später eine erst postum veröffentlichte kolorierte geologische Karte der Pyrenäen.

Ein weiterer Schub erfolgte 1801 durch den Entwurf einer geologischen Karte von England und Wales, den der englische Kanalbau-Ingenieur William Smith 
(1769-1839) vorlegte. Darauf aufbauend veröffentlichte 1819 George Bellas Greenough (1778-1855), der 1807 die Geological Society in London gründete, eine topographisch und geologisch wesentlich fortgeschrittene Karte von England und Wales. Greenough hatte in Cambridge und Göttingen Jurisprudenz studiert, wandte sich aber durch den Einfluss Blumenbachs der Geologie zu.

Schon 1826 brachte Leopold von Buch (1774-1852), ein weiterer Schüler von A. G. Werner, der kurzzeitig auch in Göttingen studiert hatte, eine geognostische Karte von Deutschland in 24 Blättern heraus, die bis 1853 insgesamt 5 Auflagen erreichte. Zumindest ab dieser Zeit war für die geologische Forschung die Erstellung geologischer Karten selbstverständlicher Usus, ohne seither wirklich prinzipielle Änderungen zu erfahren. Natürlich wurden die Kartierungen immer genauer und detaillierter; Deutschland wurde mit einem Netz von Spezialkarten im Maßstab 1:25 000 überzogen, von denen allein der in Göttingen von 1882-1907 als Ordinarius wirkende Adolf von Koenen (1837-1915) mit seinen Schülern 28 Blätter erstellte, die im Wesentlichen bis heute Gültigkeit haben. In neuerer Zeit erfuhr die geologische Kartierung besondere Impulse durch die Luftbildfotografie und durch die verschiedenen Methoden der satellitengestützten Fernerkundung.

Wie aus dem folgenden Kapitel über die Stratigraphie zu ersehen ist, besteht zwischen Kartierung und Stratigraphie, zumindest in der Zeit des Aufbruchs, eine ganz enge Interdependenz, sodass deren Entwicklungsphasen vollkommen übereinstimmen.

\section{Stratigraphie: Zeugnis der Erdgeschichte}

Die Stratigraphie beschreibt Schichten in ihrer zeitlichen Aufeinanderfolge. Dass tatsächlich Schichtstapel existieren, wurde in Einzelfällen von der Antike her bis zu Leonardo da Vinci immer wieder aufs Neue beobachtet. Eingehender befasste sich damit allerdings erst der schon genannte Nicolaus Steno Mitte des 18. Jahrhunderts. Er schuf das später so genannte Lagerungsgesetz, das heißt, er wies auf die Tatsache hin, dass Schichten eine zeitliche Abfolge enthalten und damit bei ungestörter Lagerung das Jüngere auf dem Älteren liegt.

Die Erkenntnis über einen Schichtenaufbau setzte sich bis zum Ende des 17. Jahrhunderts weitgehend durch. Die Deutung der Entstehung solcher sedimentären Abfolgen war allerdings häufig noch recht abenteuerlich und fern aller Wirklichkeit. Wir haben dies im vorausgehenden Kapitel über die Sintflut schon bei Woodward, 1695, kennengelernt. Noch ganz ähnlich waren die Vorstellungen von Johann Georg Lehmann, der 1761 durch Katharina II. nach St. Petersburg als Direktor des Kaiserlichen Museums und Professor der Chemie berufen wurde (und bereits 6 Jahre später bei einer Explosion im Chemielabor tödlich verunglückte). Auch nach Lehmann haben sich die geschichteten Gesteinsabfolgen, zusammen mit Pflanzen und Fossilien, erst nach der Sintflut abgelagert, wobei sich die Bestandteile nach der Schwere ordneten. Die geschichteten Gesteinsserien, Flözgebirge genannt, wurden von Lehmann in seinem 1756 erschienenen „Ver- 
such einer Geschichte der Flözgebirge" bereits in 30 verschiedene Schichten unterteilt. Das Flözgebirge liegt dabei auf dem älteren, schon während der Schöpfung entstandenen fossilfreien Ganggebirge. Damit kommt Lehmann zu einer ähnlichen Großgliederung, wie sie Giovanni Arduino (1713-1795), Professor der Mineralogie und Metallurgie in Venedig sowie Direktor der Bergwerke in den toskanischen und vicentinischen Gebieten, vorgeschlagen hatte. Arduino unterschied eine Abfolge von primitiven, sekundären und tertiären sowie vulkanischen Gesteinen. Die primitiven, später als primäre Serien bezeichnet, entsprechen Lehmanns Ganggebirge und die jüngeren sekundären Schichten seinem Flözgebirge. Der bei Lehmann angedeutete Widerspruch von Beobachtung und Deutung ist schon bei Martin Lister, zuletzt Leibarzt der englischen Königin Anna, zu sehen. Er vermutete, dass die verschiedenen Schichten jeweils charakteristische Fossilien enthalten, mit deren Hilfe man zu einer Art zeitlicher Ordnung kommen könnte. 1678 stellte er in einer Arbeit alttertiäre neben rezenten Muscheln dar und hob deren Ähnlichkeit hervor. Da Lister aber noch in der mittelalterlichen Tradition verhaftet war, kam er zu dem für ihn zwingenden Schluss, dass die fossilen Muscheln nicht Reste ehemaliger Lebewesen waren, sondern Naturspiele. Es blieb ihm dann nichts anderes übrig als anzunehmen, dass die verschiedenen Schichten die Fähigkeit besaßen, für sie jeweils kennzeichnende Formen hervorzubringen.

Ganz anders als Lister verwendete dessen Zeitgenosse, der berühmte englische Physiker (u. a. Konstanz der Schmelz- und Siedepunkte) Robert Hooke (16351703) seine Beobachtungen. Er schreibt 1688 über fossile Arten, die sich seiner Ansicht nach verändern können, dass ,diese Denkmäler der Natur doch sicherere Zeichen des Altertums“ sind, „als Münzen und Medaillen, deren beste kopiert oder künstlich gefertigt sein können... Und wenn es in der Tat recht schwierig ist, die Schriftzüge der Natur zu lesen, eine Chronologie aus ihnen aufzustellen und die zeitlichen Zwischenräume zu bestimmen, in denen sich diese oder jene Katastrophen oder Veränderungen ereignet haben, so ist es doch nicht unmöglich“ (zitiert nach Hölder, 1960 S. 376).

Die Gliederung der Erdgeschichte, repräsentiert durch die Schichtfolgen, ging im 18. Jahrhundert zügig voran. G. C. Füchsel, fürstlicher Leibarzt in Rudolstadt, ergänzte 1762 in seiner „Historia terrae et maris ex historia Thuringiae...“ die Lehmann'sche Gliederung des Flözgebirges. Dieses enthielt nun über dem Gangoder Grundgebirge auch das Steinkohlengebirge, das Weißgebirge bis mehlbatziges Kalkgebirge mit Flözen (Zechstein mit Kupferschiefer), überlagert vom Sandgebirge (Buntsandstein) und Muschelkalk. Anzeichen für die Sintflut werden zwar nicht ganz geleugnet, aber wenigstens reduziert bis auf den jungen, in Tälern auftretenden Tuffstein und andere zeitlich entsprechende Sedimente. Füchsel weist erstmals darauf hin, dass eine bestimmte Serie von Sedimentgestein eine bestimmte Periode in der Erdentwicklung kennzeichnet.

A. G. Werner übernahm und verfeinerte das stratigraphische System von Füchsel. Da er viele später herausragende Schüler aus ganz Europa hatte, erreichte die stratigraphische Erforschung der Erdgeschichte sehr schnell einen sehr hohen Standard. Dies gilt in gewissem Maße auch für die Biostratigraphie, da er nach 
Aussagen von Schülern lehrte, dass jede Formation ihre besonderen Fossilien hat und dass diese sich in ihrer Gestalt umso mehr den Organismen „,der gegenwärtigen Schöpfung“ nähern, je jünger die einbettende Formation ist.

Das Verdienst, der Biostratigraphie zur allgemeinen Anerkennung zu verhelfen, gebührt W. Smith. In Brinkmanns Abriss der Geologie (Band Historische Geologie, 9. Aufl., 1977, S. 5) heißt es dazu: „Um 1800 zog der englische Ingenieur William Smith aus seinen Erfahrungen bei Kanalbauten in Mittelengland den Schluss, dass die Fossilien nicht regellos in den Schichten verstaut, sondern in bestimmter Folge im Schichtprofil eingebettet sind. Mit dieser Beobachtung wurde er zum Begründer der biostratigraphischen Arbeitsweise." Dies trifft insofern zu, als erst mit den Arbeiten von W. Smith der stratigraphische Wert der Fossilien allgemeine Anerkennung fand. Es muss aber darauf hingewiesen werden, dass entsprechende Folgerungen schon vor und gleichzeitig mit W. Smith veröffentlicht wurden. Dazu gehört J. F. L. Hausmann (1782-1859), der schon Jahre vor seiner 1811 erfolgten Berufung auf den neu gegründeten Göttinger Lehrstuhl für Mineralogie und Technologie, den stratigraphischen Wert der Fossilien klar in den Vordergrund stellte. Auch viele weitere Autoren hatten den chronologischen Wert der Fossilien erkannt, diese aber eher als charakteristische Beigabe $\mathrm{zu}$ ihren lithostratigraphischen Einheiten betrachtet. Andere wiederum konzentrierten sich auf die Beschreibung von Fossilien, wobei deren stratigraphische Bedeutung höchstens eine untergeordnete Rolle spielte. So zum Beispiel die von Joh. Ernst Emman. Walch zwischen 1755 und 1775 herausgegebene und mit 274 kolorierten Tafeln versehene „Sammlung von Merkwürdigkeiten der Natur und Naturgeschichte der Versteinerungen zur Erläuterung der Knorr'schen Sammlung“. W. Smith dagegen stellte die Abfolge der Fossilien sowie deren Brauchbarkeit für die Korrelation über größere Entfernungen hin selbst in den Vordergrund.

Seit W. Smith fand somit nicht nur die Lithostratigraphie, sondern auch die Biostratigraphie allgemeine Anerkennung. Beide gemeinsam bildeten nun das Hauptfeld der geologisch-paläontologischen Forschung im 19. Jahrhundert. Im Vordergrund stand oft das rein paläontologische Interesse an den Petrefakten, wie die fossilen Pflanzen und Tiere damals meist genannt wurden. Dabei spielte es keine Rolle, wie die jeweiligen Bearbeiter die Fossilien und deren stratigraphische Abfolge deuteten: ob als Aufeinanderfolge von Neuschöpfungen, die jeweils nach Katastrophen auftraten, oder als Zeugnisse fortschreitender Entwicklung. Letzteres setzte sich nach Darwins Veröffentlichung seiner Evolutions-Theorie rasch und fast ausnahmslos durch.

Nachdem die stratigraphische Forschung einmal angestoßen war, verlief sie wie von selbst und führte zu einer rapiden Zunahme der Bearbeiter und Erkenntnisse. So kam es, dass bereits um die Mitte des 19. Jahrhunderts die erdgeschichtliche Abfolge im Groben erarbeitet war. Leider ist es in diesem Rahmen nicht möglich, auch nur die dafür wichtigsten Autoren und Arbeiten aufzuführen, geschweige denn die Vielzahl derer, die mit speziellen Studien zum Fortschritt beitrugen. Die folgenden Autoren seien deshalb nur stellvertretend genannt: Alex. 
Brongniart schrieb 1821 „Sur les caractères zoologiques des formations“, Georg Aug. Goldfuß und Georg Graf zu Münster 1826-44 über die „Petrefactae Germaniae“, Heinr. Georg Bronn 1835-38 in zwei Bänden eine „Lethaea geognostica oder Beschreibung der für die Gebirgsformationen bezeichnendsten Versteinerungen“. Adam Sedgwick erkannte 1838 das kambrische, Roderik Impey Murchison 1839 das silurische und das devonische System (in einem gesonderten paläontologischen Teil wurden auf 27 Tafeln charakteristische Fossilien des Silurs abgebildet und durch Lous Agassiz, James Sowerby und Lonsdale beschrieben). G. G. Pusch verfasste von 1833-36 eine „Geognostische Beschreibung von Polen...“, Ch. H. Pander 1830 „Beiträge zur Geognosie des russischen Reiches“, Alcide d'Orbigny 1840-55 die „Paléontologie française“ sowie 1849-52 den „Cours élémentaire de Paléontologie et de Géologie stratigraphique“ und James Hall 1844-88 die „Paleontology of New York“.

Bereits um die Mitte des 19. Jahrhunderts wurden umfassende Arbeiten wie die zuvor genannten zunehmend durch solche ergänzt, die sich auf enger begrenzte Regionen oder Abschnitte der Erdgeschichte oder auch auf spezielle Tiergruppen beschränkten. So zum Beispiel Joach. Barrande 1852-81: „Système silurien du Centre de la Bohème"; die Gebrüder Guido und Fridolin Sandberger 1850-56: „Die Versteinerungen des Rheinischen Schichtensystems in Nassau“; Friedr. Adolph Roemer 1843: „Die Versteinerungen des Harzgebirges“ und 1844: „Das Rheinische Übergangsgebirge; eine paläontologisch-geognostische Darstellung“; Fr. Aug. Quenstedt 1845-49: „Petrefaktenkunde Deutschlands, Band I: Cephalopoden“ und 1858: „Der Jura“; Adolf von Koenen, später Ordinarius in Göttingen, 1867/68: „Das marine Mitteloligocän Norddeutschlands“.

Wie die aufgeführten Arbeiten erkennen lassen, hat der im 18. Jahrhundert begonnene Aufbruch innerhalb von zirka 100 Jahren zum Durchbruch bei der Stratigraphie geführt. Die weitere Entwicklung könnte in zwei Sätzen zusammengefasst werden: 1. Im Laufe der Zeit wurde eine immer detailliertere Gliederung der Erdgeschichte erreicht. 2. Die der Stratigraphie innewohnende relative Zeitmessung konnte im 20. Jahrhundert durch die Möglichkeit der Messung des zeitlichen Zerfalls bestimmter radioaktiver Elemente in die absolute Zeitrechnung eingebunden werden (siehe Abschnitt „Aufbau und Alter der Erde“).

Die Verfeinerung der Biostratigraphie beschränkt sich naturgemäß auf die Zeit der raschen Evolution und zunehmenden Erhaltungsfähigkeit der Organismen. Dies ist das vor 543 Millionen Jahren mit dem Kambrium beginnende Phanerozoikum. Mitte des 20. Jahrhunderts wurde dieses in aufeinanderfolgende Zeiteinheiten von jeweils zirka 3 Millionen Jahren unterteilt. Inzwischen jedoch gelangt man zu Zeiteinheiten von jeweils nur 300000 Jahren. Mit Hilfe von sich schnell entwickelnden Artengruppen oder/und Anwendung aller verfügbaren Methoden, also bei holostratigraphischem Vorgehen, können selbst bis zu 10-fach kleinere Zeiteinheiten ausgeschieden werden.

Die hoch auflösende Feinstratigraphie brachte auch auf dem Gebiet der Evolution große Fortschritte, da diese nun in entsprechend kleine Schritte aufgelöst werden kann. 


\section{Evolution: Voraussetzung für Fortschritt}

Der Gedanke einer Entwicklung der Lebewesen taucht seit der Antike immer wieder auf, allerdings ohne vor dem 18. Jahrhundert in das jeweils herrschende Lehrgebäude aufgenommen zu werden, geschweige denn dieses nachhaltig zu verändern. Es wurde schon erwähnt, dass Aristoteles höhere Lebewesen auf die im Schlamm erzeugten Ursprungsformen zurückführte. Dies ließ sich jedoch im Mittelalter nicht mit der biblischen Schöpfungsgeschichte vereinbaren. Umso überraschender ist deshalb der Versuch, eben aus der biblischen Geschichte eine Entwicklung abzuleiten. Sir Walter Raleigh (1552-1618), der in Ungnade gefallene Admiral und ehemalige Günstling der englischen Königin Elisabeth I., der später durch Jakob I. zur Hinrichtung verurteilt wurde, schrieb in seiner 13-jährigen Haft im Tower von London eine 5-bändige Weltgeschichte. Darin leitete er auch ab, dass die Arche Noah zu klein war, um neben den Lebewesen der Alten Welt auch die der Neuen Welt aufzunehmen. Letztere müssen sich also nach der Sintflut aus den Ersteren entwickelt haben.

Etwas fundierter, nämlich auf Züchtungsversuchen beruhend, nahm John Ray (1628-1705) eine Translation bei Pflanzen an. Die Fossilien ließ er allerdings aus der Meerestiefe durch aufbrechende Wasserfluten an den Strand befördern, von wo sie dann durch Meerkatzen auf die Berge getragen wurden. Demgegenüber erkennt man bei Matthias Hale, 1685, einen klaren wissenschaftlichen Ansatz, nämlich Beobachtungen bei der Haustierzüchtung. Daraus und aus der Aufeinanderfolge ähnlicher, aber nicht gleich gestalteter Fossilien schloss er auf eine Veränderlichkeit der Arten. Diese führte er, weit vorausblickend, auf Wechsel in den Umweltbedingungen zurück.

Dass die allgemeine Anerkennung einer Entwicklung der Arten noch weit über ein Jahrhundert dauerte, ist aus der noch vorherrschenden Bindung an die Bibel zu verstehen. So berichtet Leibniz 1700 in seiner Protogaea, warum „manche“ Zeitgenossen eine Entwicklung annehmen. „Aber solches widerspricht den heiligen Schriftstellern, von denen abzuweichen sündhaft ist" (zitiert nach Hölder 1960, S. 376). Eine derartige Formulierung ist offensichtlich eine zu dieser Zeit gängige Camouflage für die Tatsache, dass man selbst zu den scheinbar kritisierten „manchen“ Zeitgenossen gehört.

Im Laufe des 18. Jahrhunderts und besonders in dessen zweiter Hälfte, verzeichnen wir eine enorme Zunahme von Veröffentlichungen über Petrefakten oder Schichtfolgen mit jeweils kennzeichnenden Fossilien. Dies bewirkte eine sich selbst verstärkende sammlerische Tätigkeit und in zunehmendem Maße eine problemorientierte Beschäftigung mit den damit verbundenen Fragen. So ist es nicht verwunderlich, dass sich der Verdacht über eine Veränderlichkeit der Arten verstärkte. Beispiele dafür sind die später noch zu besprechenden Autoren De Maillet, Soulavie, Palissy, Guettard und De Buffon.

Den Durchbruch zu einer Evolutions-Theorie errang jedoch erst Jean Baptiste de Lamarck (1774-1829), der 1794 mit 20 Jahren als Professor der Zoologie für Insekten, Würmer und Mikroorganismen an den Jardin des Plantes, das spätere 
Musée Nationale d'Histoire Naturelles, berufen wurde. An seiner Seite stand Etienne Geoffroy de Sainte-Hilaire (1772-1844), der am Ägypten-Feldzug Napoleons teilnahm und von diesem später zum wissenschaftlichen Inspekteur der Pyrenäen-Halbinsel eingesetzt wurde. Im Gegensatz zu Cuvier, der die Analogien heranzog, beschäftigte sich Sainte-Hilaire mit Homologien, zum Beispiel von Fisch-Flossen und Tetrapoden-Beinen. Dies führte ihn zur Annahme eines einzigen Grundbauplans für die „Urform“ aller Tiere, entsprechend der „Urpflanze“ Goethes. Wenn man sich vergegenwärtigt, dass sich die Erkenntnis über die Veränderlichkeit der Arten damals häufig auf die Interpretation von Züchtungen beschränkte, dass außerdem die Diluvianer, unter anderen der am selben Institut tätige Cuvier, noch das Feld beherrschten und selbst die Naturspiel-Hypothese noch Anhänger hatte, dann ist die Leistung der damaligen Evolutionisten und vor allem die Lamarcks zumindest ebenso hoch einzuschätzen wie die ein halbes Jahrhundert später veröffentlichte Evolutions-Theorie von Charles Darwin (1809-92) und dessen Konkurrenten Alfred Russel Wallace (1823-1913). Letzterem kommt allerdings das Verdienst zu, das Vorhandensein und die Bedeutung biogeographischer Unterschiede erkannt zu haben.

Darwin, dessen Theorien-Gebäude auf seinen Erfahrungen und Erkenntnissen fußt, die er bei der Forschungsreise mit der Beagle gewonnen hatte, stand in enger Beziehung zu Charles Lyell (1797-1875), der auch als Begründer der modernen Geologie bezeichnet wird. Seine 1830 bis 1835 veröffentlichten „Principles of Geology“ machte er Darwin schon während dessen Reise zugänglich. Er wies darin darauf hin, dass die heute das Bild der Erde prägenden Kräfte dieselben sind, die auch in früheren Erdzeitaltern im Verlauf von ungeheuerlichen Zeiträumen wirkten. Allerdings leugnete Lyell in seinen „Principles“ noch eine Evolution der Lebewesen, ließ sich dann aber durch Darwin völlig überzeugen und wurde zum glühenden Anhänger von Darwins Selektions-Theorie. Es mag Lyell ähnlich ergangen sein wie dem englischen Zoologen Thomas Huxley (1825-95), der nach der Lektüre des 1859 erschienenen Werkes Darwins „On the origin of species“ sagte: „Was bin ich doch dumm, dass ich daran nicht gedacht habe“.

Die Abstammungslehre Darwins wurde in kürzester Zeit anerkannt und führte sofort zu umfangreichen Studien an Arten-Ketten und an der Stammesgeschichte aller Gruppen der Lebewesen. Der jetzt häufig benützte Begriff Neo-Darwinismus schließt die ganz erstaunliche Tatsache ein, dass Darwins Theorie auch heute noch gültig ist, obwohl Darwin von den später gemachten Erkenntnissen nichts wissen konnte, das heißt von den zwar schon zu Darwins Lebzeiten aufgestellten, aber erst 1900 neu entdeckten Vererbungsgesetzen des Augustiner-Mönches Georg Johannes Mendel (1822-1884), bis zur Molekularbiologie und der Dechiffrierung genetischer Kodizes.

Selbstverständlich erfährt eine so lange existierende Theorie Modifikationen, kritische Ergänzungen oder Abstriche in einzelnen Punkten. Aber alle Versuche, die Darwinsche Theorie als überholt oder gar ungültig zu erklären, sei es durch Wissenschaftler mit falschen oder überzogenen Fakten oder sei es durch die hoffentlich bald der Selektion anheim fallende Indoktrination bei Kreationisten, 
scheiterten. Darwins Vererbungslehre ist nach wie vor der Schlüssel für das Verstehen der schon Milliarden Jahre andauernden Entwicklung des irdischen Lebens.

\section{Die Erde: Werden und Gestaltung}

Wie zuvor schon angedeutet wurden seit der Antike immer wieder Überlegungen darüber angestellt, wie alt die Erde ist, welchen inneren Aufbau sie hat und welche inneren und äußeren Kräfte sie gestalteten beziehungsweise auch heute noch wirksam sind. Antworten auf diese Fragen waren zum großen Teil erst nach der hier behandelten Zeit des Auf- und Durchbruchs zu einer Wissenschaft möglich. Dies liegt zum einen daran, dass zuerst Grundfragen nach dem Schichtenbau (Stratigraphie), der räumlichen Anordnung (Paläogeographie) und der Evolution geklärt werden mussten. Zum anderen wurden die notwendigen chemischen und physikalischen Methoden erst relativ spät, nämlich in der ersten Hälfte des 20. Jahrhunderts, entwickelt.

Ein typisches Beispiel ist die Frage nach dem Alter der Erde. Buffon vermutete in der zweiten Hälfte des 18. Jahrhunderts, dass für die Bildung und Dauer der von ihm aufgestellten 7 Epochen die biblischen 6000 Jahre unzureichend waren. Selbst das von ihm aus der Abkühlungszeit glühender Kugeln abgeleitete Alter der Erde von 74832 Jahren würde nicht ausreichen, sondern man müsste mit mindestens 100000 oder gar mit mehr als 1 Million Jahren rechnen. Mit zunehmender Akzeptanz der Stratigraphie und der Evolution wurden immer längere Zeitspannen vermutet. Ein Beweis für das wirkliche Alter von zirka 4,5 Milliarden Jahren war erst im 20. Jahrhundert möglich, nachdem der Zerfall bestimmter radioaktiver Elemente zur Zeitmessung herangezogen werden konnte. Schon Jahrzehnte davor, 1898, hatte das Physiker-Ehepaar Marie und Pierre Curie mit der Entdeckung der Radioaktivität die Voraussetzung dafür geschaffen.

Auch der Schalenaufbau der Erde konnte erst geklärt werden, nachdem im 20. Jahrhundert die geophysikalischen Methoden zur seismischen Durchleuchtung der Erde entwickelt waren. Noch gegen Ende des 17. Jahrhunderts sahen die Modellvorstellungen, z. B. die von Kircher, nicht viel anders aus als in der Antike. Und bis weit in das 19. Jahrhundert hinein beherrschte der Streit zwischen Neptunisten und Plutonisten beziehungsweise Vulkanisten die Diskussion. Die nach den griechischen Göttern der Unterwelt beziehungsweise des Feuers benannten Plutonisten beziehungsweise Vulkanisten nahmen an, dass an der Bildung der Erdkruste hauptsächlich die aus geschmolzener Materie hervorgegangenen Gesteine beteiligt waren, auf denen dann im Ozean gebildete Sedimente abgelagert wurden. Ein Hauptvertreter der Plutonisten war James Hutton (1726-97). Dem traten A. G. Werner und seine Schüler entgegen, nach deren neptunistischer Ansicht die Gesteine der Erdkruste aus den Ozeanen ausgeschieden wurden. Viele dieser Gruppe anerkannten später zunehmend die Basalte als vulkanische Produkte. Der Widerspruch der beiden Lager löste sich bereits im 2. Drittel des vorletzten Jahrhunderts durch neue Erkenntnisse über die Petrographie der Gesteine auf. 
Ein weiterer Komplex betrifft die Geodynamik. Deren Erscheinungsformen, wie zum Beispiel Hebung und Senkung, Klüftung, Überschiebung, Faltung etc., waren Ende des 19. Jahrhunderts schon Gegenstand intensiver Untersuchungen und Diskussionen. Ungeklärt blieb die Ursachenfrage, die auf vielerlei Weise, aber, wie wir heute wissen, falsch beantwortet wurde. Ganz neue Aspekte ergaben sich aus der 1913 veröffentlichten Theorie des Geophysikers und Polarforschers Alfred Wegener (1880-1930). Aus dem passgenauen Verlauf der sich gegenüberliegenden Küsten Südamerikas und Afrikas schloss er, dass beide Kontinente früher eine Einheit bildeten, dann zerbrachen und sich durch eine Kontinentaldrift zunehmend voneinander entfernten. Diese Theorie wurde sehr skeptisch aufgenommen und weitgehend viele Jahrzehnte in Zweifel gezogen. Als dann in den darauf folgenden 60er Jahren paläomagnetische Anomalie-Streifen am mittelatlantischen Rücken auf eine Ausweitung des Ozeanrückens (see floor spreading) deuteten, wurde Wegeners Theorie bestätigt und 1965 durch Wilson und andere das geotektonische Konzept der Plattentektonik entwickelt.

Wie ersichtlich, fand die Klärung geologischer Fragen, welche den Erdaufbau und die Geodynamik betreffen, gegenüber dem Komplex Kartierung/Stratigraphie/Evolution erst mit einer zeitlichen Verzögerung statt. Dies zeigt zweierlei: zum Einen, dass zur Lösung von Problemen zuvor gewisse erkenntnismäßige Grundvoraussetzungen geschaffen werden müssen, und zum Anderen, dass die Entwicklung einer Wissenschaft, wenn sie nicht rein spekulativ, sondern wissenschaftlich begründet angestoßen ist, zum „Selbstläufer“" wird. Die herausgestellte Reihenfolge gilt heute allerdings nur noch in beschränktem Maße: die Kenntnis der räumlichen Erfassung und der zeitlichen Abfolge ist bereits auf so hohem Niveau, dass darauf auch für viele weitere Fragen aufgebaut werden kann. Sie bilden zwar nach wie vor die Basis vieler Forschungszweige, haben aber durch ihren großen Fortschritt die Spitzenposition in der Forschung an neue Zweige der Geologie und Paläontologie abgegeben.

\section{Korrelation zwischen geowissenschaftlicher und kultureller Entwicklung}

Aus den vorausgegangenen Darstellungen ist hinsichtlich der Entwicklung der Geowissenschaften eine klare Zweiteilung zu konstatieren: Der vor zirka 250 Jahren beginnenden Dämmerung und dem Aufbruch hin zu einer modernen Wissenschaft gehen Jahrtausende der geowissenschaftlichen Finsternis voraus, in denen zwar die montanistische Seite genutzt wurde, aber die auch in der Antike schon auftretenden erdgeschichtlichen Erkenntnisse nicht zu weiterführenden Untersuchungen und Vorstellungen führten. Worauf ist das zurückzuführen? Für die Antwort darauf müssen mehrere Faktoren berücksichtigt werden, die ich mit den Stichworten Befangenheit, Repression, Autoritätsgläubigkeit sowie Bedarf kennzeichnen möchte. Mit Befangenheit ist ganz allgemein das Gebundensein an 
das jeweilige Niveau der kulturellen Entwicklung gemeint. Dazu gehören auch die anderen genannten Faktoren, die wegen ihrer Bedeutung aber extra hervorgehoben werden sollen. Dies ist zum einen die Repression, das heißt die bewusste Unterdrückung von allem, was die bestehenden Machtverhältnisse gefährden könnte und damit im Gegensatz zur verantwortlichen Freiheit des Denkens und Handelns steht. Ein nicht zu unterschätzendes Hemmnis für weitere Entwicklung ist die kritiklose Autoritätsgläubigkeit per se, aber auch deren gezielte Ausnützung. Der Bedarf, zum anderen, ergibt sich zumeist aus dem Bestreben der Weiterentwicklung sowohl bei den Erkenntnissen als auch bei den materiellen Gegebenheiten; mit anderen Worten: der Bedarf entsteht aus dem Wunsch, die sich fortlaufend erneuernde Neugierde zu befriedigen sowie aus dem Bestreben, die materiellen Grundlagen zu optimieren.

\section{Zeit der Finsternis}

Im Prolog und dem darauf folgenden Abschnitt wurde bereits dargelegt, dass die erstmals auftretende Gewinnung und Verarbeitung von Metall, zusammen mit der etwa gleichzeitigen Heranbildung von Schriften, zu dem 4. großen Umbruch in der Menschheit führten. Das Aufspüren der dazu notwendigen Ausgangsmaterialien wurde von speziell Kundigen, die wir als Prospektoren bezeichnen können, durchgeführt. Man kann wohl davon ausgehen, dass sich frühzeitig für die Prospektion förderliche Kenntnisse herausbildeten und sich im Laufe der Generationen aus der zunehmenden Erfahrung heraus vermehrten. Auf jeden Fall spielte dieser im weiteren Sinn geologische Teil anfangs wohl einen ganz entscheidenden Einfluss auf die kulturelle Entwicklung. Im Laufe der Zeit verlor er aber seine vorrangige Bedeutung. Offensichtlich war es nicht notwendig, die Bildungsbedingungen der Erze und die Einordnung der Lagerstätten in die geologischen Gegebenheiten eingehend zu erforschen. Die Montangeologie verharrte damit über 5000 Jahre hinweg bis in das 19. Jahrhundert in der Funktion einer Beschafferin von Erz und Kohle, ohne die Voraussetzung und Notwendigkeit zu bieten, sich zu einer Wissenschaft zu entwickeln.

So wie die Montangeologie hatten auch die anderen erdgeschichtlichen Vorstellungen in der Antike keinerlei Einfluss auf die kulturelle Entwicklung. Die in einigen Schöpfungsgeschichten des Altertums enthaltenen vagen Vorstellungen über die Entstehung und Entwicklung der Erde und ihrer Bewohner gaben keinen Anlass zu weitergehenden Überlegungen oder gar Forschungen. Einen Einfluss auf die kulturelle Entwicklung gewannen sie allerdings einige Millenien später, als die christliche Kirche die Bibel - und damit auch die Genesis - als göttliche Wahrheit und damit als ihr nicht diskutierbares Fundament deklarierte und verteidigte.

In der griechischen Antike blieben die Gedanken über die Entstehung der Erde und der Lebewesen auf dem spekulativen Niveau der alten religiösen Mythologien stehen und wurden durch die weitgehend philosophisch behandelte Frage nach dem Urstoff beziehungsweise den Urstoffen überprägt. Vorstellungen über den Aufbau der Erde beruhten nur teilweise auf Beobachtungen in der Natur, 
wobei dann die sichtbare Vulkantätigkeit und die unermesslichen Meere im Vordergrund standen. Die daraus entwickelten Vorstellungen von feuerflüssiger Materie unter der Gesteinsschale oder im Kern der Erde sowie riesigen, wassergefüllten Kammern blieben in verschiedenen, häufig recht obskuren Modifikationen bis in die Neuzeit hinein bestehen.

Bezüglich Veränderungen an der Erdoberfläche gab es, wie im ersten Kapitel beschrieben, durchaus Deutungen, die zumindest in eine ausbaubare Richtung wiesen: Überschwemmungen des Festlandes durch das Meer, worauf die eingebetteten Versteinerungen hinwiesen; Materialtransport durch Flüsse bis hin zur Bildung eines Deltas; Bildung vulkanischer Inseln sowie Veränderungen der sichtbaren Erdkruste durch Erdbeben und spezielle Vorgänge, die wir heute als Tektonik bezeichnen.

Es ist nicht unbedingt anzunehmen, dass in der Antike das seltene Befasstsein mit geologischen Phänomenen auf Repressionen zurückzuführen ist. Es hat solche zwar auf anderen Gebieten gegeben, wie wir zum Beispiel bezüglich Sokrates wissen, der wegen angeblicher Götterlästerung, tatsächlich aber wohl eher wegen seiner Kritik an der weltlichen Ordnung Athens verurteilt wurde und durch den Trunk aus dem Schierlingsbecher starb. Manch anderer kritische Gelehrte wurde direkt oder indirekt vertrieben. So Xenophanes aus Kolophon, der nach Elea ging. Dabei spielten seine Erkenntnisse über die Entstehung geologischer Schichten wohl keine Rolle, sondern der von ihm vertretene Monotheismus. Der weitgereiste Pythagoras wanderte von Samos nach Kroton in Unteritalien aus. Dabei dürften die ganz beachtlichen geologischen Erkenntnisse, die ihm einige Jahrhunderte später Ovid zuschrieb, kaum eine Rolle gespielt haben. Ovid selbst und die anderen Naturforscher der augusteischen Zeit waren gegenüber ihren Vorläufern aus der griechischen Antike in ihren geologischen Vorstellungen noch weiter fortgeschritten, ohne dadurch besonderen Repressionen ausgesetzt zu sein.

Für die vorchristliche Antike bedeuteten die erreichten erdgeschichtlichen Erkenntnisse und spekulativen Vorstellungen offensichtlich keine Gefahr für die weltliche und geistliche Obrigkeit. Dass sich in diesen rund 500 Jahren trotzdem nicht einmal die Anfänge einer Geowissenschaft herausbildeten, muss also andere Gründe gehabt haben. Zu diesen gehört sicher, dass sich die Bildung des größten Teiles der Bevölkerung auf die Ausbildung für ihren Lebensberuf beschränkte, also für die Landwirtschaft und das Kleingewerbe. Hinzu kamen allenfalls Begründungen für das soziale und religiöse Verhalten. Damit bestand für diese Schicht weder der Bedarf noch die Möglichkeit, philosophische Betrachtungen anzustellen oder Naturstudien zu betreiben, die über den eigenen Bedarf hinausgingen.

Auch für die gebildete Elite bestand offensichtlich kein Bedarf an der Erweiterung erdgeschichtlicher Erkenntnisse. Eine Ausnahme bildete in gewisser Weise die Frage nach der Stellung der Erde im Weltensystem. Der heliozentrischen Hypothese Heraklits stellte Aristoteles die Erde als Weltzentrum gegenüber. Obwohl nachfolgend Aristarchos wieder die Sonne in die Mitte stellte, ver- 
half vier Jahrhunderte später Ptolemaeus dem geozentrischen Weltbild zu einer anderthalb Jahrtausende überdauernden Geltung.

Vereinfacht kann für die Antike festgestellt werden, dass erdgeschichtliche Erkenntnisse für die kulturelle Entwicklung keine Rolle spielten. Die für entsprechende Forschungen notwendige Neugierde und damit ein Bedarf für die Erklärung gewisser geologischer Gegebenheiten fehlte weitgehend. Sie trat nur sporadisch auf und dann kennzeichnenderweise besonders bei Autoren, die weitgereist waren und damit eine Vielzahl geologischer Erscheinungen beobachten konnten. Repressionen, die manches Mal durchaus auch stimulierend auf das unterdrückte Wissensgebiet wirken können, waren nicht vorhanden. Offensichtlich berührten die spärlichen erdgeschichtlichen Erkenntnisse weder die Götterwelt unter Zeus noch unter Jupiter. Dies änderte sich grundlegend erst im Mittelalter, lange nachdem sich die christliche Religion in Europa durchgesetzt hatte.

Bestimmend für erdgeschichtliche Fragen waren im christlichen Mittelalter die biblische Genesis, also die einmalige, unveränderliche Schöpfung aller Lebewesen durch Gott, sowie die mosaische Sintflut. Alle andersartigen Erklärungsversuche wurden rigoros unterdrückt. Eine besondere Rolle spielte auch die Dominanz und Verehrung des großen antiken Lehrers Aristoteles, nachdem Avicenna zu Beginn des 11. Jahrhunderts dessen Werke in das Latein, die Wissenschaftssprache des Mittelalters, übersetzt hatte und nachdem in der Scholastik versucht wurde, die Lehre des Aristoteles mit der der christlichen Kirche zu vereinen. Dies führte zum Teil zu einer kritiklosen Übernahme seiner Theorien, wie zum Beispiel durch das mittelalterliche Universalgenie Albertus Magnus, der deshalb auch als „Affe des Aristoteles" bezeichnet wurde.

Die wirkungsvollste Innovation für die kulturelle Entwicklung, und dabei besonders auch für die Wissenschaften, war der Mitte des 15. Jahrhunderts von Gutenberg (Johannes Gensfleisch, ca. 1400-1467) entwickelte Buchdruck mit beweglichen Lettern. Dies ermöglichte gegenüber zuvor eine relativ preiswerte, rasche und weite Verbreitung von neuen Ideen und Erkenntnissen, ebenso wie von Spekulationen und Disputationen. Der damit initiierte Aufbruch vom Mittelalter in die Neuzeit bedeutet gleichzeitig den nach unserer Zählung 5. großen Umbruch in der kulturellen Entwicklung der Menschheit. Er wurde durch viele innerhalb weniger Jahrzehnte erbrachte Neuerungen und Entdeckungen verstärkt.

Die Kulturwende war die Zeit, in der unter anderen Michelangelo Buonarotti (1475-1564), Leonardo da Vinci (1452-1519), Albrecht Dürer (1471-1528) oder Lucas Cranach d. Ä. (1472-1553) in erstaunenswerter Kreativität die frühe Phase der Renaissance in der bildenden Kunst verkörperten. Es war auch die Zeit der maßgebenden Wissenserweiterung über die Geographie unserer Erde und deren Stellung im Weltsystem: Christoph Columbus (1451-1543) entdeckte 1492 Amerika, Vasco da Gama (1469-1524) fand 1497/98 den Seeweg nach Indien und Nikolaus Kopernikus (1473-1543) erbrachte 1514 den Beweis für das heliozentrische Planetensystem. Und es war die Zeit, in der Martin Luther (1483-1546) mit der Bekanntmachung seiner Thesen 1517 die Reformation einleitete. 
Man sollte annehmen, dass dieser 5. große kulturelle Umbruch auch die Erforschung der Erdgeschichte in Gang setzte. Für die Tatsache, dass dies nicht geschah, lassen sich wohl eine Reihe von Gründen aufführen. Entscheidend aber war, dass es im Hinblick auf die Erdwissenschaften nicht zutraf, „dass der Mönch Martin Luther vor fünfhundert Jahren die Christenheit aus dem Mittelalter erlöste“ (Der Spiegel, 51/2003). Ganz im Gegenteil: für Luther war die Bibel und damit die Genesis, also die einmalige Erschaffung der Welt durch Gott, die unveränderliche Grundlage des Glaubens. Luther und zum Teil noch mehr die anderen Reformatoren wie Ulrich Zwingli (1484-1531) und Johann Calvin (1509-1564) waren in dieser Beziehung Fundamentalisten und Kreationisten, für die eine langsame Entwicklung der Erde und ihrer Lebewesen undenkbar und damit zu verwerfen und zu bekämpfen war. Insofern wurde das Mittelalter fortgesetzt und es bestand in dieser Beziehung kein grundsätzlicher Unterschied zur weiterhin andauernden repressiven Politik der katholischen Kirche.

Man muss sich vergegenwärtigen, dass noch im Jahre 1600 Giordano Bruno (1548-1600) in Rom als Ketzer verbrannt wurde, da er das kopernikanische Weltbild zu einer Weltanschauung erweiterte, in der Gott in der Welt und die Welt in Gott erhalten ist. Er vertrat auch die Ansicht, dass bestimmte Phänomene auf dem Land nicht durch die biblische Sintflut verursacht wurden, sondern auf Wechsel von Land und Meer hindeuteten. 1614, also erst 100 Jahre nach der Erstveröffentlichung, verbot Papst Paul V. die heliozentrische Idee des Kopernikus. Deshalb musste der Astronom Galileo Galilei (1564-1642) seine wohl begründete Anhängerschaft an Kopernikus 1633 vor einem inquisitorischen Tribunal widerrufen. Nördlich der Alpen konnte dagegen Johannes Kepler (15711630) seine Planetengesetze 1619 unangefochten veröffentlichen. Er schuf sich damit so großes Ansehen, dass er seine Mutter, die als Hexe angeklagt war, vor dem Feuertode retten konnte.

Neue Gedanken nicht nur über die Stellung der Erde, sondern auch über deren Geschichte, standen weiterhin unter dem christlichen Bann. Gegen Ende des 16. Jahrhunderts starb Bernard Palissy (1509-1589) in der Pariser Bastille, da er als Hugenotte seinem reformierten Glauben nicht ebenso abschwören wollte, wie er das für seinen 1580 veröffentlichten „Discours admirable de la nature...“ tat. Dort hatte er den Standpunkt vertreten, dass versteinerte Muscheln und Schnecken von ehemals wirklich lebenden Tieren stammen.

Einen besonderen Weg, der Repression durch die Obrigkeit zu entgehen, ging Benoit de Maillet. Er wählte die Form des Dialogs zwischen einem der Kirchenlehre getreuen französischen Missionar und einem indischen Philosophen, der neben kuriosen Phantasien die fortschrittlichen, also ketzerischen Gedanken vertrat: Fossilien lebten einst an den Fundorten unter normalen Meeresbedingungen; viele fossile Arten sind heute unbekannt; die biblische Flut war nicht universell, sondern nur lokal; die Landpflanzen entwickelten sich durch Transmutation aus Meerespflanzen. Als weitere Vorsichtsmaßnahme verfügte er, dass die Arbeit erst postum unter dem Anagramm Telliamed veröffentlicht werden soll, was dann 1748 geschah. 
Selbst noch gegen Ende des 18. Jahrhunderts wurden Widerrufe erzwungen. So von Etienne Guettard durch die französische königliche Zensur. Außer der erwähnten ersten geologischen Karte von Frankreich veröffentlichte er zahlreiche neue Ideen, die im Widerspruch zu den herrschenden Ansichten standen: die Brauchbarkeit von Fossilien für die Gliederung der Erdgeschichte und die sich daraus ergebende Folgerung, dass nicht nur eine einzige Flut, also die biblische, geschah, sondern eine Vielzahl von Meereseinbrüchen. Unter Androhung der Untersagung wissenschaftlicher Arbeit veröffentlichte er einen Widerruf, der allerdings eher nichtssagend war.

Noch besser mit der Zensur umzugehen verstand George Louis Leclerc de Buffon (1707-1788), der 1731 Intendant des Jardin des Planets in Paris wurde, und damit einer der Vorgänger Cuviers. Er hatte 1749 seine „Theorie de la Terre“ und 1778 über die „Epoques de la Nature“ veröffentlicht. Darin und in weiteren Publikationen wandte er sich gegen die Sintflut-Hypothese und zeigte, dass die Fossilien früherer Epochen ausgestorben waren. Buffon gelang es allerdings, alle seine bahnbrechenden Erkenntnisse in eine solche Form zu kleiden, dass die vor der französischen Revolution gegen ihn tätige Zensur wirkungslos blieb.

Dass die Vorsicht vor der Zensur und all die geleisteten Widerrufe nicht aus Überzeugung, sondern zum Selbstschutz erfolgten, darf, ja muss angenommen werden. Weit schwieriger dagegen ist die Frage, ob die zur selben Zeit wirkenden Vertreter des vis plastica-Mythos oder der Sintflut-Sage aus Opportunismus diesen Hypothesen anhingen oder ob sie davon tatsächlich überzeugt waren und sich ohne eigenes Bemühen der mehrheitlich kursierenden Deutung anschlossen. Man könnte für jede dieser Möglichkeiten begründete Beispiele anführen, ebenso wie auch dafür, dass die durch Erziehung und weitere Umwelteinflüsse vermittelten religiösen Dogmen wichtige, für sich eigentlich überzeugende Einsichten zu blockieren vermögen. Als eklatantes Beispiel dafür kann Cuvier angeführt werden, von dem Honoré de Balzac (1799-1850) in seinem 1831 erschienenen Roman „La Peau de Chagrin“ poetisch schwärmt: „Ist Cuvier nicht der größte Dichter unseres Jahrhunderts? Der unsterbliche Naturforscher ... hat Welten neu erstehen lassen aus gebleichten Knochen, er hat - wie Katmos - Städte wieder aufgerichtet aus Zähnen, ... er hat Geschlechter von Riesen wieder aufgefunden in dem Fußabdruck eines Mammuts“. Aber trotz der diesem Ruhm zugrundeliegenden Leistungen und obwohl an seinem Institut Lamarck und Sainte-Hilaire die Evolution der Lebewesen lehrten und er selbst wie sein Vorgänger Buffon nicht mit einer einmaligen Sintflut auskam, sondern mehrere bis zahlreiche Katastrophen annehmen musste, beharrte er auf der Unveränderlichkeit der Arten. Wie ist dies möglich? Repressionen durch die Obrigkeit, zu der er zuletzt selbst zählte, waren in der Zeit des Aufbruchs, zu dem er selbst Entscheidendes beitrug, nicht zu erwarten. Man ist deshalb versucht, diese auf die Frage der Evolution bezogene Unbeweglichkeit religiöser Befangenheit, das heißt in seinem Fall der streng calvinistischen Erziehung, zuzuschreiben.

Zusammenfassend kann für die finstere Zeit der Geowissenschaften folgendes festgestellt werden: Wenn man vom Bergbau absieht, spielten erdgeschichtliche 
Kenntnisse in der Antike keine Rolle. Im darauf folgenden christlichen Mittelalter und in der frühen Neuzeit bis hinein in das 18. Jahrhundert nahm das naturhistorische Interesse zwar zu, wurde aber dann unterdrückt, wenn es wirklich oder auch nur scheinbar im Widerspruch zur Bibel stand. Obwohl trotzdem in Einzelfällen Anfangserkenntnisse auftraten, blieben diese zu ihrer Zeit für die kulturelle Entwicklung nur insofern von Bedeutung, als ihre Ablehnung und Unterdrückung den Aufbruch zu einer fortgeschrittenen Gesellschaft ganz wesentlich verzögerte.

\section{Dämmerung, Auf- und Durchbruch}

Fragen wir uns nun, wie es dazu kam, dass nach den rund zwei Jahrtausenden der Ignoranz und Unterdrückung der immer wieder auftauchenden naturhistorischen Erkenntnisse nun in verhältnismäßig kurzer Zeit der Auf- und Durchbruch zu wissenschaftlicher Höhe gelang. Häufig begnügt man sich mit der Feststellung, dass es ,in der Luft lag“ oder nun „die Zeit reif dafür“" war. Versuchen wir doch, diese Floskeln zu hinterfragen, das heißt herauszufinden, welche Faktoren sich dahinter verbergen.

Bei der Ursachenfrage muss berücksichtigt werden, dass historische Abläufe komplexe Vorgänge sind. Dabei können sich sowohl Fortschritt als auch Stillstand auf einem bestimmten Gebiet fördernd oder hemmend auf völlig andere Bereiche auswirken und durch Rückkopplung das Ausgangs-Gebiet selbst wieder beeinflussen. Wir müssen auch damit rechnen, dass eine Innovation auf einem Wissensfeld bis zu dessen Auswirkung auf einen anderen Bereich eine gewisse Zeit benötigt. Deshalb sollten wir die kulturelle Entwicklung nicht nur während des Aufbruchs, sondern auch in der Zeit davor darauf durchforschen, ob und wie weit dort Aspekte auftraten, die in einer hier als Dämmerung bezeichneten Zeitspanne mit zum Aufbruch beitrugen.

Die in der zu betrachtenden Zeit hervorragendste Änderung in der kulturellen Evolution ist sicher die Aufklärung im weiteren Sinne. Wir müssen dabei zurückgehen bis René Descartes (1596-1650), dem französischen Philosophen und Mathematiker. Obwohl dieser, erzogen in einer Jesuiten-Schule, an der traditionellen Verbindung von Theologie und Wissenschaft festhielt, kann er als Begründer des modernen Rationalismus betrachtet werden. Er fordert in seiner mechanistischen Weltsicht „klare und distinkte“ Anschauungen sowie ein Zurückgehen auf die einfachsten Einsichten, zu denen die Erkenntnis cogito ergo sum (ich denke, also bin ich) als grundlegende Gewissheit gehört.

Die nach Descartes eingetretene geistige und wissenschaftliche Entwicklung hin zu der Zeit der Aufklärung und in dieser selbst sind durch viele, die kulturelle Entwicklung entscheidend fördernde Namen gekennzeichnet. Eine kleine Auswahl davon, von Descartes bis in das 19. Jahrhundert reichend, sei, da sie für sich selbst sprechen, kommentarlos aufgeführt: Sir Isaac Newton (1643-1727), Gottfried Wilhelm Freiherr von Leibniz (1646-1716), David Hume (1711-1776), Carl von Linné (1707-1778), Immanuel Kant (1724-1804), Pierre Simon Marquis de Laplace (1724-1804), Georg Friedrich Wilhelm Hegel (1770-1831). Wichtige 
technologische Meilensteine waren in dieser Zeitspanne: Tiegelgussstahl, James Watts Dampfmaschine 1765, erste Spinnmaschinen zwischen 1763 und 1771 (die nach Friedrich Engels [1820-1895] den Beginn der industriellen Revolution kennzeichnen), erstes Dampfschiff 1807, Zylinder-Schnelldruckpresse 1812, erste Eisenbahn 1825. Deutlich treten auch wichtige gesellschaftliche und politische Veränderungen und Ereignisse hervor: Nordamerikanische Unabhängigkeitserklärung 1776, französische Revolution 1789, Abschaffung des Sklavenhandels zwischen 1792 und 1850, Säkularisation zwischen 1789 und 1802.

Leibniz versuchte noch, die mechanistische Naturerklärung Descartes' mit den religiösen Vorstellungen in Übereinstimmung zu bringen. Demgegenüber trennte Kant uneingeschränkt die Religion von der Philosophie. Für ihn war die Vernunft der Schlüssel, dem Menschen zum „Ausgang aus einer selbstverschuldeten Unmündigkeit“ zu verhelfen. Aus dieser Zielsetzung heraus befasste er sich auch mit einer Allgemeinen Naturgeschichte des Himmels, in der er die später so genannte Kant-Laplace'sche Nebular-Theorie entwickelte, die in ihrer Grundaussage heute noch gültig ist. Da die Arbeit 1755 unmittelbar vor dem Siebenjährigen Krieg (1756-63) erschien, fand sie erst nach ihrer Wiederentdeckung durch A. von Humboldt im 19. Jahrhundert die gebührende Beachtung. In der 1790 erschienenen „Kritik der Urteilskraft“ leitet Kant von der Analogie vieler Merkmale der Lebewesen ab, dass dies auf eine wirkliche Verwandtschaft hinweist und damit auf eine ,gemeinsame Urmutter“. Daraus ergibt sich nach Kant für die Erdgeschichtler die Aufgabe, aus den Erdschichten den Entwicklungsgang des Lebens abzuleiten.

Die in diesem Abschnitt aufgeführten Namen, technischen Fortschritte und gesellschaftlichen Veränderungen sind sowohl Basis als auch Resultat der Aufklärung und in vielfacher Weise miteinander verwoben. Mit der Aufklärung wurde das Repressions-Potential der Kirche gegenüber naturwissenschaftlichen Ergebnissen weitgehend geschwächt oder sogar aufgehoben. Als symbolisches Beispiel für diesen Umbruch mag Giraud Soulavie dienen. Als hochrangiger Priester begeisterte er sich für die französische Revolution, trat dem Klub der Jacobiner bei und vertrat außerdem die Veränderlichkeit der Arten.

Eine große Rolle spielte neben den genannten Faktoren die im 18. Jahrhundert schnell zunehmende Anzahl von Veröffentlichungen. Die Konfrontation mit den auch qualitativ besseren Darstellungen von Fossilien und geologischen Phänomenen regte immer mehr Zeitgenossen an, sich damit zu beschäftigen, sich kritisch damit auseinanderzusetzen und die Kommunikation mit anderen Naturhistorikern zu pflegen. Vorrang hatte dabei, entsprechend einer Darstellung von Agostino Scilla, 1752, „die Beobachtung als Bändiger vager Spekulationen“ (aus Hölder, 1960, Taf. 1). Dazu gehörte auch die zunehmende Zahl der Forschungsreisen wie die von Vater und Sohn Forster als Teilnehmer der Weltumsegelung durch Cook, später im 20. Jahrhundert unter anderen die von A. v. Humboldt, A. R. Wallace und Ch. Darwin. Aus dem zunehmenden Interesse heraus entstehend und gleichzeitig selbst fördernd wirkend waren die Naturalienkabinette, die sich später zu naturhistorischen Museen entwickelten, sowie die in 
der ersten Hälfte des 19. Jahrhunderts gegründeten geologischen Gesellschaften mit ihren Zeitschriften, und schließlich die Einrichtung geologischer Dienste, wie zum Beispiel 1873 die Preußische Geologische Landesanstalt.

Die Entwicklung der Geowissenschaften von der Zeit der hier so genannten Dämmerung an bis zu ihrem Durchbruch zeigt einige allgemeine, wohl auch auf viele andere Gebiete der kulturellen Entwicklung anwendbare Aspekte. Die unvorstellbar lange Zeit des mangelnden allgemeinen Interesses und der Repression wird erst durch eine geistige und gesellschaftliche Neuordnung beendet, wobei die erstere, also in unserem Fall die Aufklärung, die initiale kulturelle Veränderung hervorruft. Die spätere Entwicklung wird gekennzeichnet durch eine enge Vernetzung aller geistigen, gesellschaftlichen und technischen Bereiche. Sie erfährt dabei eine zunehmende Beschleunigung. In diesen Prozess ist die Entwicklung der Erdwissenschaften sowie deren zunehmende Bedeutung mit einbezogen. Dies beschränkt sich nicht nur auf die rein technischen und wirtschaftlichen Aspekte, sondern beeinflusst oder verursacht in hohem Maße auch die Veränderungen in den gesellschaftlichen Bereichen, einschließlich der religiösen und philosophischen Vorstellungen.

\section{Geowissenschaften heute: gewichtiger Faktor der kulturellen Entwicklung}

Die Geowissenschaften umfassen heute ein weites naturwissenschaftliches Feld. Sie haben zwar im Laufe der Zeit einige Teilgebiete abgetreten, auch wenn sie in diesen noch mehr oder weniger intensiv mitwirken. Dies gilt besonders für die morphologische Gestaltung der Erdoberfläche, für die Bodenkunde, in gewissem Maße auch für die Geologie des jüngsten Erdzeitalters, des Quartärs, und vor allem für die Paläoanthropologie, also die Entwicklung des Menschen und seiner Vorfahren. Auf der anderen Seite wirken sie intensiv auf zahlreichen Gebieten mit, die im Allgemeinen anderen naturwissenschaftlichen Zweigen zugeordnet werden. Manche davon wurden von Geowissenschaftlern angestoßen oder zumindest sehr stark beeinflusst. Dies gilt vor allem für diejenigen Bereiche, die nicht nur für die Gegenwart, sondern ebenso für die Entwicklung der Erde und des Lebens von Bedeutung sind, also zum Beispiel Klima, Ozeanographie, marine und terrestrische Ökologie.

Es braucht wohl nicht näher herauszustellen sein, dass die Geowissenschaften für viele Bereiche der Zivilisation unverzichtbar sind. Dies betrifft zum Beispiel die Prospektion der meisten Naturstoffe wie Erze, Mineralien, Steine und Erden, fossile Brennstoffe wie Kohle, Erdöl und Erdgas, und im besonderen Maße Wasser. Darüber hinaus tragen die Geowissenschaften auch die Verpflichtung, diese Naturstoffe verantwortlich zu erschließen und zu benutzen.

Die derzeitig und künftig größte, aber auch schwierigste Aufgabe der Menschheit ist die Bewahrung einer lebenswerten Umwelt. Besonders schwierig ist diese Aufgabe aus zwei Gründen. Zum Einen muss sie die wirksame Zügelung individueller und institutioneller Egoismen betreiben, soweit diese dem 
erweiterten Kant'schen Imperativ zuwiderhandeln, also keine Rücksicht nehmen auf die Erhaltung günstiger Lebensbedingungen für die heutigen Lebewesen und deren Nachkommen. Dies betrifft nicht nur die ganz eklatanten Vergehen wie zum Beispiel die völlig rücksichtslose und gewissenlose Überfischung und Kontamination der Meere oder den ungezügelten Verbrauch fossiler Energieträger. Es gilt auch für die ganze Spanne von großen bis kleinen „Umweltsünden“, die im Einzelfall kaum wirksam, in ihrer Summierung jedoch ein hohes Gefährdungspotential bilden. Die andere Schwierigkeit bei der Bewältigung der Aufgabe ist, dass sie nicht durch eine einmalige, kurzfristige Anstrengung zu lösen ist, sondern vielmehr eine andauernde Auseinandersetzung mit den Problemen und mit deren wirksamer Lösung erfordert.

Die Geowissenschaften sollten bei der Bewältigung der Zukunftsaufgaben eine führende Rolle einnehmen. Sie sind auf fast allen relevanten Teilgebieten maßgebend aktiv. Außerdem sind sie per se sowohl mit vernetzten Systemen als auch mit Langzeitprozessen vertraut. Die Geowissenschaften bringen damit alle Voraussetzungen mit, unsere Umwelt in Gegenwart und Zukunft lebenswert zu erhalten. Dies ist gleichzeitig die Basis für eine weitere positive kulturelle Entwicklung.

\section{Epilog}

Unvoreingenommen und streng betrachtet war die Kenntnis über die Geschichte der Erde und deren Bewohner über Jahrtausende hinweg bis in das 18. Jahrhundert hinein annähernd gleich null. Daran änderte die seit dem Beginn der Metallzeit notwendige bergbauliche Tätigkeit ebenso wenig wie die auf die Erfassung und Beschreibung der Mineralien beschränkte Mineralogie. Auch die in dieser für unsere Wissenschaft finsteren Zeit gelegentlich auftretenden richtungsweisenden Erkenntnisse änderten daran nichts. Sie wurden im besten Falle ignoriert, meist aber unterdrückt oder aus Furcht vor Repressionen so erfolgreich geheim gehalten, dass ihre Existenz erst im 18. oder 19. Jahrhundert bekannt wurde. Die Geognosie hatte also über Jahrtausende hinweg keinen prägenden Einfluss auf die kulturelle Entwicklung. Man kann im Gegenteil eher annehmen, dass eben diese Unkenntnis über die Geschichte der Erde und des Lebens eine der wesentlichen Ursachen dafür war, dass die religiösen und gesellschaftlichen Strukturen über Millennien hinweg extrem dogmatisch verharren konnten.

Die Repressionen gingen direkt oder indirekt zumeist von den Religionen aus. Im europäisch, vorderasiatisch oder ägyptisch beeinflussten Raum waren es in der Antike diverse Polytheismen und zeitlich daran anschließend die jüdischen, christlichen und islamischen Monotheismen. Die in deren Namen ausgeübten Repressionen dienten der Erhaltung der Macht nicht nur der Vertreter dieser Religionen, sondern missbräuchlich auch anderer gesellschaftlicher beziehungs- 
weise politischer Kräfte. Ganz Entsprechendes gilt natürlich auch für andere, auf nichtreligiösen Weltanschauungen beruhenden Organisationen.

Unkritische Autoritätsgläubigkeit und Fundamentalismus sind für die Emanzipation der Menschheit die gefährlichsten Hemmnisse. Die Forderung nach deren Beseitigung gilt auch in der Gegenwart. Es ist inakzeptabel, dass Kreationismus an Schulen gelehrt sowie versucht wird, noch 150 Jahre nach Darwin die Evolutionslehre aus den Schulen zu verdrängen. Für die Verfechter dieser allen biologischen und paläontologischen Erkenntnissen Hohn sprechenden Betrachtungen, vor allem aber für die dafür politisch Verantwortlichen, gilt, mehr als höflich ausgedrückt, die von Leonardo da Vinci für die seinerzeitigen Vertreter der vis plastica geprägte Feststellung, dass ,eine solche Meinung nicht in Gehirnen mit großem Denkvermögen besteht“. Es ist zu hoffen, dass die Religionen diejenigen Elemente, die zwar aus deren Entstehungszeit heraus verständlich sind, aber allen modernen Erkenntnissen widersprechen, zurechtrücken und vielmehr ihre positiven, insbesondere ethischen Werte als ihre Basis auffassen, darstellen und begründen.

Die an alle Religionen und Weltanschauungen gerichtete Forderung zur Anerkennung und entsprechenden Berücksichtigung naturwissenschaftlicher Erkenntnisse ergibt sich aus dem im späteren Verlauf der Aufklärung entstandenen modernen Weltbild. Ganz wesentlich daran beteiligt waren die neuen Erkenntnisse über den Aufbau und die Geschichte unseres Planeten sowie über die schon mehrere Milliarden Jahre ablaufende Entwicklung der Lebewesen. Die Geowissenschaften traten damit endlich aus ihrer weitgehenden Bedeutungslosigkeit für die kulturelle Entwicklung heraus und beeinflussten diese seither in einem zwar häufig nicht vordergründig erkennbaren, in Wirklichkeit jedoch hoch relevanten Ausmaß.

Grundlage für die zuvor schon erwähnte Emanzipation der Menschheit ist die überzeugte Akzeptanz des Kant'schen Imperativs, allerdings erweitert durch die Erkenntnis der alleinigen Verantwortung der Menschen für das globale Ökosystem in Gegenwart und Zukunft, also für die Umwelt, in der und von der wir und zukünftige Generationen leben.

Die Geowissenschaften können dazu aus verschiedenen Gründen einen wichtigen Beitrag leisten. Zum einen befassen sie sich mit vielen für die Umweltbedingungen wichtigen Teilgebieten. Damit sind für ihre Vertreter die Voraussetzungen dafür gegeben, notwendige Problemlösungen in holistischer Betrachtungsweise anzugehen. Zum anderen sind sie gewöhnt, in ihre Überlegungen die Wirkung über lange Zeiträume, das heißt über eine Vielzahl von politischen Wahlperioden hinaus, einzubeziehen. Damit sind sie in der Lage, sowohl für natürlich ablaufende Prozesse in unserer Umwelt als auch für solche, die artifiziell in Gang gesetzt werden, deren langfristige Folgen und die sogenannte Nachhaltigkeit beabsichtigter Veränderungen abzuschätzen.

Die geowissenschaftliche Forschung wird sich schwerpunktmäßig am Bedarf der Gesellschaft orientieren müssen. Es wäre jedoch falsch, sich nur nach der ökonomischen Wirksamkeit der erzielten Ergebnisse auszurichten. Auch aus zweckfreier Grundlagenforschung ergeben sich Erkenntnisse, die nicht nur die 
theoretischen Grundlagen der Geowissenschaften wesentlich erweitern, sondern in ihrer praktischen Anwendung die kulturelle Entwicklung vorantreiben, unter anderem auch einfach durch die Bereicherung unseres Wissens.

Wissen wollen um des Wissens willen ist eine ernsthafte Beschäftigung und kein eitles Verlangen.

Albertus Magnus (im 13. Jabrhundert)

\section{Literatur}

Blumenbach, J. F. (1796-1810): Abbildungen naturhistorischer Gegenstände, Nr. 1-100, Heinrich Dieterich, Göttingen

Blumenbach, J. F. (1816): Handbuch der Naturgeschichte, 9. Ausgabe, zweyte Abtheilung, Kath. Gräffer und Härter, Wien, 325 S.

Dougherty, F. W. P. (1984): Commercium epistolicum J. F. Blumenbachii, Niedersächsische Staats- und Universitätsbibliothek, Göttingen, 220 S.

Hölder, H. (1960): Geologie und Paläontologie in Texten und ihrer Geschichte. In der Reihe „Orbis academicus, Problemgeschichten der Wissenschaft in Dokumenten und Darstellungen“ (Hrg. F. Wagner \& R. Brodführer), Verlag Karl Alber, Freiburg/München, XVIII, 566 S.

Hölder, H. (1989): Kurze Geschichte der Geologie und Paläontologie, SpringerVerlag Berlin Heidelberg, 244 S.

Selle, G. v. (1937): Die Georg-August-Universität zu Göttingen 1737-1937, Vandenhoek \& Ruprecht, Göttingen, 398 S.

Walliser, O. H. \& Alberti, L. (1987): Die Geschichte der Geologie und Paläontologie in Göttingen. In: Georgia Augusta, Nachrichten der Universität Göttingen: 73-76

Wendt, H. (1965): Ehe die Sintflut kam, G. Stalling, Oldenburg, 392 S.

Zittel, K. A. v. (1899): Geschichte der Geologie und Paläontologie bis Ende des 19. Jahrhunderts. In der Reihe „Geschichte der Wissenschaften in Deutschland. Neuere Zeit“, R. Oldenbourg, München/Leipzig, 23. Band, XI, 868 S. 


\section{Anschriften der Autoren}

Prof. Dr. Manfred Schidlowski

Max-Planck-Institut für Chemie

Otto-Hahn-Institut

Abteilung Biogeochemie

Becherweg 27

55128 Mainz

Tel. (0 61 31) 3 05-4 21

biogeo@mpch-mainz-mpg.de

Prof. Dr. Reinhard Gaupp

Friedrich-Schiller-Universität Jena

Institut für Geowissenschaften

Burgweg 11

07749 Jena

Tel. (0 36 41) 948620

gaupp@geo.uni-jena.de

\section{Prof. Dr. Sharon Webb}

Geowissenschaftliches Zentrum der

Universität Göttingen

Abteilung für Experimentelle und

Angewandte Mineralogie

Goldschmidtstraße 1

37077 Göttingen

Tel. (05 51) 39-38 61

swebb@gwdg.de

\section{Prof. Dr. Bent Tauber Hansen}

Geowissenschaftliches Zentrum der

Universität Göttingen

Abteilung Isotopengeologie

Goldschmidtstraße 3

37077 Göttingen

Tel. (05 51) 39-97 06

bhansen@gwdg.de
Prof. Dr. Martin Kappas

Universität Göttingen

Geographisches Institut

Abteilung Kartographie, GIS \&

Fernerkundung

Goldschmidtstraße 5

37077 Göttingen

Tel. (05 51) 39-80 71

mkappas@gwdg.de

\section{Dr. Annette Broschinski}

Niedersächsisches Landesmuseum

Hannover

Naturkunde-Abteilung

Willy-Brandt-Allee 5

30169 Hannover

Tel. (05 11) 98 07-8 64

annette.broschinski@

pop-h.niedersachsen.de

Prof. Dr. Klaus Weber

Geowissenschaftliches Zentrum der

Universität Göttingen

Abteilung Strukturgeologie \&

Geodynamik

Goldschmidtstraße 3

37077 Göttingen

Tel. (05 51) 39-79 30

kweber@gwdg.de

\section{Prof. Dr. Otto H. Walliser}

Geowissenschaftliches Zentrum der

Universität Göttingen

Abteilung Geobiologie

Goldschmidtstraße 3

37077 Göttingen

Tel. (05 51) 39-79 60

uggp@gwdg.de 
